\title{
Salt forms of sulfadiazine with alkali metal and organic cations.
}

2 Gemma Campbell, Rebecca Fisher, Alan R. Kennedy,* Nathan L. C. King and Rebecca Spiteri

Westchem, Department of Pure \& Applied Chemistry, University of Strathclyde, 295 Cathedral Street, Glasgow G1 1XL, Scotland

Correspondence email: a.r.kennedy@strath.ac.uk

\section{Abstract}

The structures of four salt forms of sulfadiazine (SDH) with alkali metal cations are presented. Three contain the deprotonated SD anion. These are the discrete complex $\left[\mathrm{Li}(\mathrm{SD})\left(\mathrm{OH}_{2}\right)_{2}\right],(\mathrm{I})$, and the coordination polymers $[\mathrm{Na}(\mathrm{SD})]_{\mathrm{n}}$, (II), and $\left[\mathrm{K}(\mathrm{SD})\left(\mathrm{OH}_{2}\right)_{2}\right]_{\mathrm{n}}$, (III). The Na complex (II) is a three-dimensional coordination polymer whilst the $\mathrm{K}$ complex (III) has two crystallographically independent $\left[\mathrm{K}(\mathrm{SD})\left(\mathrm{OH}_{2}\right)_{2}\right]$ units per asymmetric unit, $Z^{\prime}=2$, and gives a twodimensional coordination polymer whose layers propagate parallel to the crystallographic $a b$ plane. The different bonding modes of the SD anion in these three complexes is discussed. Structure (IV) contains protonated $\mathrm{SDH}_{2}$ cations and the Orange $\mathrm{G}(\mathrm{OG}), \mathrm{C}_{16} \mathrm{H}_{10} \mathrm{~N}_{2} \mathrm{O}_{7} \mathrm{~S}_{2}$, dianion in a structure with formula $\left[\mathrm{SDH}_{2}\right]_{2}\left[\mathrm{Na}(\mathrm{OG})\left(\mathrm{OH}_{2}\right)_{4}\right]_{2} \cdot 3 \mathrm{H}_{2} \mathrm{O}$. The $\left[\mathrm{Na}(\mathrm{OG})(\mathrm{OH})_{4}\right]_{2}$ dimers have antiparallel naphthol ring structures joined through two Na centres that bond to the hydrazone anions through the $\mathrm{O}$ atoms of the ketone and sulfonate substituents. The structures of the salts formed on reaction of SDH with 2-aminopyridine and ethanolamine are also presented as $\left[\mathrm{C}_{5} \mathrm{H}_{7} \mathrm{~N}_{2}\right][\mathrm{SD}],(\mathrm{V})$, and $\left[\mathrm{HOCH}_{2} \mathrm{CH}_{2} \mathrm{NH}_{3}\right][\mathrm{SD}] \cdot \mathrm{H}_{2} \mathrm{O}$, (VI), respectively. Structure (V) features a heterodimeric $R_{2}^{2}(8)$ hydrogen bond motif between the cation and the anion whilst structure (VI) has a tetrameric core of two cations linked by a central $R_{2}^{2}(10)$ hydrogen bonded motif which supports two anions linked to this core by $R_{3}{ }^{3}(8)$ motifs.

Keywords: crystal structure, active pharmaceutical ingredients, salt selection, sulfadiazine, sulfa drugs

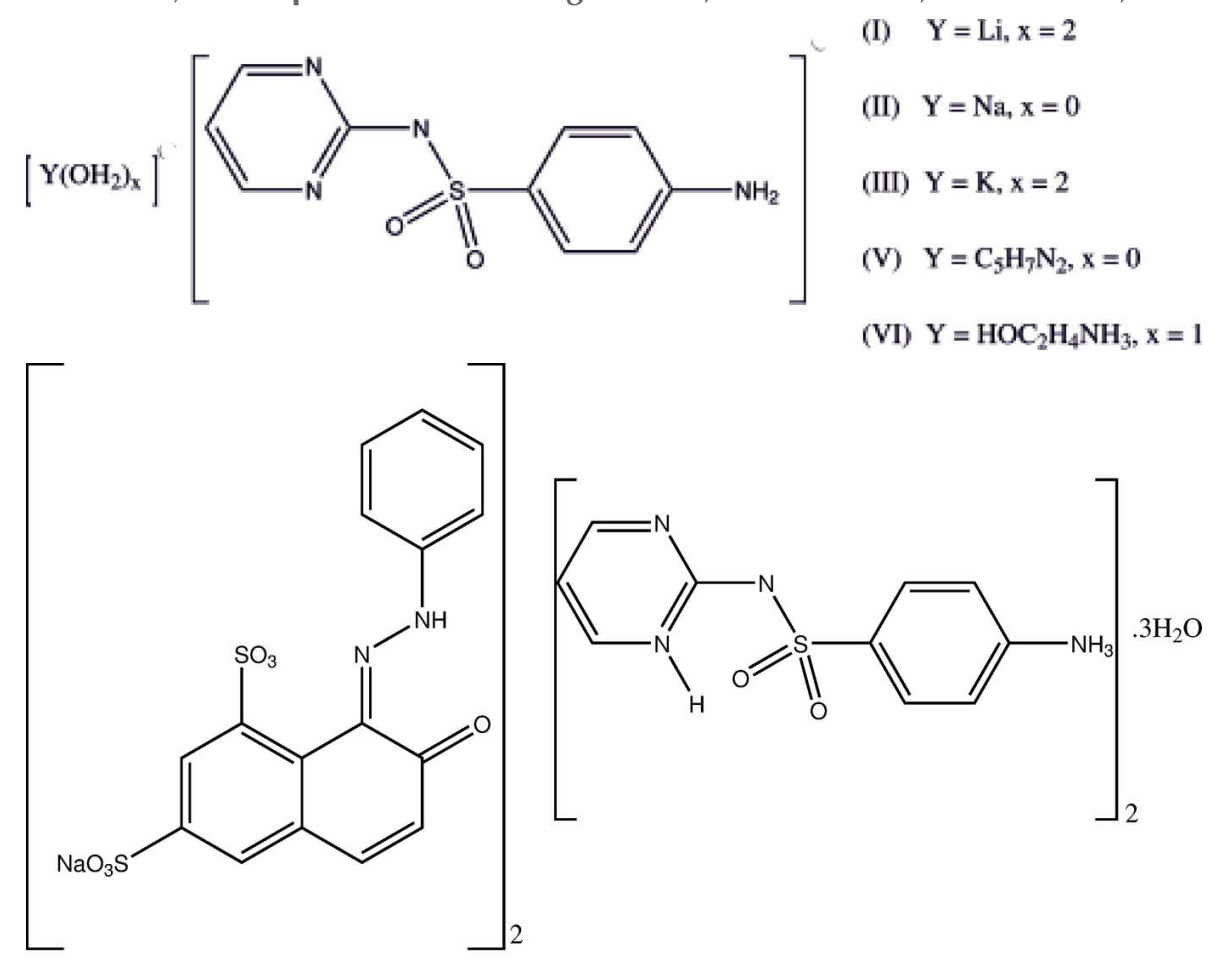

(IV) 


\section{Comment}

The active pharmaceutical ingredient (API) 4-amino- $N$-(pyrimidin-2-yl)-benzenesulfonamide is commonly known as sulfadiazine (SDH) and is a well known antibiotic. Common variants are its $\mathrm{Ag}(\mathrm{I})$ complex which is used in creams and impregnated medical devices, and its Na salt which is used intravenously (Fisher et al., 2003; Ghedini et al., 2017; Mohseni et al., 2016; Preskey \& Kayes, 1976). SDH is amphoteric, allowing salt formation reactions to be carried out with both acids and bases. This is a pharmaceutically useful as salt formation is the commonest route used to modify the performance critical material properties (e.g. aqueous solubility, melting point or mechanical properties) of APIs (Stahl \& Wermuth, 2008).

Structural studies of protonated $\mathrm{SDH}_{2}$ cations as a variety of salt forms have been published, (e.g. Pan et al., 2013; Buist et al., 2014) as have studies of cocrystal phases featuring neutral SDH (e.g. Elacqua et al., 2013). Structures of the deprotonated SD anion are also well represented in the literature. These include the structure of the commercially utilized $\mathrm{Ag}(\mathrm{I})$ complex (Baenziger \& Struss, 1976) and of many transition metal complexes, especially those involving the heavier first row transition metals Co, Ni, Cu and Zn (e.g. Shi et al., 2015; Sun et al., 2016; Pan et al., 2012). Salt structures of SD with organic cations are also well known (e.g. Elacqua et al., 2013; Heren et al., 2006). Somewhat strangely, there are very few structural studies of s-block metal complexes of SD. As far as we are aware, the only known s-block metal structure is that of a Ca salt form (Tommasino et al., 2011). Given the ubiquity of s-block metal salt usage in pharmaceutical materials in general, and the long-standing commercial use of [Na][SD] in particular, this seemed an odd omission (Stahl \& Wermuth, 2008; Preskey \& Kayes, 1976). The current study adds to our knowledge of sulfadiazine structural chemistry by reporting the structures of three alkali metal salt forms of SD with Li, Na and K, structures (I), (II) and (III) respectively, as well as the structure formed when the sulfonated azo dye sodium Orange G (OG) crystallizes in the presence of $\mathrm{SDH}$ to give a form containing both $\mathrm{Na}$ and $\mathrm{SDH}_{2}$ cations, (IV). Finally, the structures of two new organic salt forms (V) and (VI), prepared by reaction of SDH with the bases 2-aminopyridine and ethanolamine, are also presented for comparison.

The structure of the lithium salt (I) was found to consist of a simple discrete coordination compound of type [Li(SD) $\left(\mathrm{OH}_{2}\right)_{2}$ ], see Figure 1. Extensive hydrogen bonding creates a three-dimensional hydrogen bonding network, Table 3. The $\mathrm{SD}$ anion acts in a chelating fashion to form a 6-membered [LiOSNCN] ring through $\mathrm{Li}$ to $\mathrm{O}$ and $\mathrm{Li}$ to heterocyclic $\mathrm{N}$ bonds. Thus although the Li coordination geometry is tetrahedral, the bond angles are considerably distorted due to the small bite angle of the chelate (range 90.91 (18) to 126.9 (2) ${ }^{\circ}$ ), see Table 2 for details of bond lengths and angles. This $\mathrm{O}, N$ chelation mode is unusual. Discrete d-block metal complexes of SD normally bond to metal centres through a $\mathrm{N}, N$ chelate utilizing both the ring and sulfamide $\mathrm{N}$ atoms (e.g. Shi et al., 2015; Sun et al., 2016; Pan et al., 2012). The same is true of the Ca salt (Tommasino et al., 2011). The polymer $[\mathrm{Zn}(\mathrm{SD})]_{\mathrm{n}}$ does feature a similar $\mathrm{O}, N$ chelation mode (Yuan $e t$ al., 2001) and the dimeric species $\left[\mathrm{Cu}_{2}(\mathrm{SD})_{4}\right]$ can be described as containing $\mathrm{Cu}-\mathrm{O}$ bonds, but these are very long $(2.55$ to $2.75 \AA$ ) and form 4-membered chelate rings through interactions with the sulfamide $\mathrm{N}$ atom rather than the 6membered ring involving the aromatic ring observed for (I) (Shi et al., 2015). The structure of the pharmaceutically important coordination polymer $[\mathrm{Ag}(\mathrm{SD})]_{\mathrm{n}}$ does contain the $\mathrm{O}, N$ chelation mode seen for (I) with the polymer propagating through both the common $\mathrm{N}, N$ chelation mode and the $\mathrm{O}, N$ mode (Baenziger \& Struss, 1976).

Like the Ag complex, the structure of anhydrous $[\mathrm{Na}(\mathrm{SD})]_{\mathrm{n}}$ (II) forms a coordination polymer through each SD anion making two chelating interactions with two metal centres. One such interaction forms a 6-membered NaOSNCN ring with a $\mathrm{O}, N$ bonding mode and the other forms a NaNCN ring with a $\mathrm{N}, N$ mode. The coordination polymer is further connected by $\mathrm{NaONaO} 4$-membered rings, see Figs 2 and 3. Thus the atoms N1, N2, N3 and O1 bond to Na centres and form one-dimensional coordination chains that propagate parallel to the crystallographic $a$ direction. Unlike the Ag 
complex, in (II) the amine group of the ligand also takes part in bonding. This gives 6 coordinate Na centres, Table 4 . This last interaction type links the individual chains through amine to $\mathrm{Na}$ bonds and gives the overall three-dimensional coordination polymer shown in Fig. 4. We are aware no other examples of anionic SD bonding to metal through its $\mathrm{NH}_{2}$ tail. The coordination polymer is supported by amine to $\mathrm{SO}_{2} \mathrm{~N}=\mathrm{H} \cdots \mathrm{O}$ hydrogen bonds as described in Table 5 .

The $\mathrm{K}$ salt (III) is a dihydrate with two crystallographically independent $\left[\mathrm{K}(\mathrm{SD})\left(\mathrm{OH}_{2}\right)_{2}\right]$ units per asymmetric unit, $Z^{\prime}=$ 2, see Fig. 5. Of the 4 independent water molecules, two $(\mathrm{O} 2 \mathrm{~W}$ and $\mathrm{O} 3 \mathrm{~W})$ act as terminal ligands whilst $\mathrm{O} 1 \mathrm{~W}$ bridges between $\mathrm{K} 1$ and $\mathrm{K} 1^{\prime}\left({ }^{\prime}=1-x, 1-y, 1-z\right)$ and $\mathrm{O} 3 \mathrm{~W}$ interestingly bridges between three $\mathrm{K}$ centres (K1, $\mathrm{K} 2$ and $\mathrm{K} 1{ }^{\prime \prime}$ where " $=-x, 1-y, 1-z$ ), see Table 6 for details. The two independent SD anions also have different bonding modes, the ligand containing $\mathrm{O} 3$ utilizes both of its $\mathrm{O}$ atoms and 3 of its $\mathrm{N}$ atoms to bond between 4 separate $\mathrm{K}$ centres. Each $\mathrm{N}$ atom bonds to only one $\mathrm{K}$ centre with the $\mathrm{O}$ atoms both bridging between two $\mathrm{K}$ centres. Like the $\mathrm{Na}$ and $\mathrm{Ag}$ species, above, this ligand features both $\mathrm{N}, N$ 4-membered ring forming and $\mathrm{O}, \mathrm{N}$ 6-membered ring forming chelation modes. Perhaps surprisingly, the other SD anion adopts a bonding mode that forms only two donor to K contacts and bonds to only one $\mathrm{K}$ centre. Atoms $\mathrm{O} 1$ and N1 (the sulfamide $\mathrm{N}$ atom) bond to K2. This $\mathrm{O}, \mathrm{N}$ [KOSN] 4-membered ring forming bonding mode is not seen for other s-block metals with SD but can be observed in the structure of $\left[\mathrm{Cu}_{2}(\mathrm{SD})_{4}\right]$ (Shi et al., 2015). See Table 12 for a summary of the different bonding modes adopted by SD with alkali metals. A final difference between the two SD ligands is conformational. The SD anion that bonds to multiple $\mathrm{K}$ centres has the same conformation as that found for the $\mathrm{SD}$ anions in all the other structures reported herin. That is, one of the $\mathrm{SO}_{2} \mathrm{O}$ atoms lies close to the plane of the aniline ring and the pyrimidine ring is syn to this $\mathrm{O}$ atom. However, the $\mathrm{SD}$ anion that makes only 2 bonds to $\mathrm{K} 2$ has an alternative conformation where it is the amide $\mathrm{N}$ atom and not an $\mathrm{O}$ atom that lies closest to the plane of the aniline ring (N1S1C5C6 $=17.7(4)^{\circ}$ ). The coordination bonds combine to give two-dimensional coordination polymers that propagate parallel to the $a b$ plane and gives the layered structure shown in Fig. 6, with organic bilayers and inorganic layers alternating along the crystallographic $c$ direction. Hydrogen bonds from the amine group link between neighbouring two-dimensional coordination polymers, Table 7.

Crystallized from SDH in the presence of acid and the sodium salt of OG, (IV) has the general formula $\left[\mathrm{SDH}_{2}\right]_{2}\left[\mathrm{Na}(\mathrm{OG})\left(\mathrm{OH}_{2}\right)_{4}\right]_{2} \cdot 3 \mathrm{H}_{2} \mathrm{O}$, Fig. 7. We are unaware of any other structure containing both a metal cation and cationic $\mathrm{SDH}_{2}$. There are 4 independent water ligands bound to Na1. These are ordered but the non-coordinated water molecules are not and have been modelled over 3 crystallographic sites, each with site occupancy factor of 0.5 . In common with other sulfonated azo species based on naphthol units, the dye adopts the hydrazone tautomeric form with protonation at $\mathrm{N} 6$ of the $\mathrm{N}=\mathrm{N}$ group (Kennedy et al., 2012). This leads to characteristic lengthening (e.g. $\mathrm{N}=\mathrm{N}, \mathrm{C}-\mathrm{C}$ ) and shortening (e.g. $\mathrm{O}-\mathrm{C}, \mathrm{N}-\mathrm{C}$ ) of relevant bonds compared to similar azo species. Compare values in Table 8 with e.g. 1.253 (2) and 1.418 (3) $\AA$ for $\mathrm{N}=\mathrm{N}$ and $\mathrm{N}-\mathrm{C}$ bond lengths in a typical azo species (Kennedy et al., 2001). The dimeric unit is shown in Fig. 8 and is crystallographically centrosymmetric, $Z^{\prime}=0.5$. The naphthol units lie antiparallel to each other with an octahedral $\mathrm{Na}$ centre at each end. In addition to the 4 terminal water ligands, each $\mathrm{Na}$ centre bonds to the ketone group of one anion and to atom $\mathrm{O} 7$ of a sulfonate group of a second anion thus creating the dimeric unit. See Tables 8 and 9 for bonding parameters. There is some degree of $\pi$ to $\pi$ stacking across this dimer as is indicated by a minimum $\mathrm{C}$. $\mathrm{C}$ distance of 3.482 (2) $\AA$ (between $\mathrm{C} 11$ and the $\mathrm{C} 15$ atom at $-x, 1-y,-z$ ). Orange $\mathrm{G}$ is exceptional amongst sulfonated azo colourants in having being widely studied crystallographically. Structures are known for its s-block metal salts, for its complexes with transition metals and for salt forms with organic cations (Kennedy et al., 2006; Ojala et al., 1994b; Kennedy et al., 2010; Ojala et al., 1994a). However, the dimeric unit observed here is not seen for other s-block metal salt forms of OG. The closest motif occurs in the $\mathrm{Ag}(\mathrm{I})$ complex of $\mathrm{OG}$, where a similar dimer forms part of a larger polymeric coordination network (Kennedy, et al., 2006). The sulfadiazine cation is found to have three $\mathrm{H}$ atoms bound to $\mathrm{N} 4$, the aniline group, and one $\mathrm{H}$ atom bound to $\mathrm{N} 3$ of the pyrimidine ring. In contrast, previous work had found that 
$\mathrm{SDH}_{2}$ cations adopted this tautomeric form with simple anions but with sulfonate anions gave the alternative tautomer with protonation at the amide $\mathrm{N}$ atom (Buist et al., 2014). The geometry of the $\mathrm{SDH}_{2}$ cation is somewhat different from those of the SD anions in the other structures presented. Conformationally, it is again an $\mathrm{O}$ atom that lies closest to the plane of the aniline ring, but in contrast to the $\mathrm{SD}$ anions this $\mathrm{O}$ atom is anti to the pyrimidine ring. An expected small increase in $\mathrm{S}-\mathrm{N}$ bond length is also seen for $\mathrm{SDH}_{2}$ as compared to the $\mathrm{SD}$ anions (Elacqua et al., 2013; Buist et al., 2014). Another feature common in other structures with $\mathrm{SDH}_{2}$ cations is a centrosymmetric $R_{2}^{2}(8)$ hydrogen bonded dimer of cations formed utilizing amide and pyrimidine $\mathrm{N}$ atoms (Buist et al., 2014). This motif is retained in (IV). Structure (IV) has a layered structure with hydrophylic/inorganic layers parallel to the $b c$ plane alternating with hydrophobic/organic layers.

Formed from the reaction of SDH with 2-aminopyridine, structure $(\mathrm{V})$ is that of $\left[\mathrm{C}_{6} \mathrm{H}_{4} \mathrm{~N}(\mathrm{H}) \mathrm{NH}_{2}\right][\mathrm{SD}]$, Fig 9. It features a heterodimeric $R_{2}^{2}(8)$ hydrogen bond between the cation and the amide $\mathrm{N}$ atom and one pyrimidine ring $\mathrm{N}$ atom of the SD anion, Fig 10. There are also anion to anion interactions with the amine group of the SD anion donating two hydrogen bonds to $\mathrm{O}$ atoms of two neighbouring SD anions. A final hydrogen bond is of type $\mathrm{N}-\mathrm{H} \cdots \mathrm{N}$ and is donated by the pyridine amine group to the SD amine group, see Table 10 for details. Unusually this leaves one $\mathrm{N}$ atom of the pyrimidine ring with no hydrogen bonding interaction. The resulting structure has layers formed of heteroaromatic rings (both pyridine and pyrimidine) alternating with layers of aniline groups, these layers lie parallel to the crystallographic $a c$ plane.

Structure (VI) was obtained on the reaction of SDH with ethanolamine and is that of the hydrate, $\left[\mathrm{HOCH}_{2} \mathrm{CH}_{2} \mathrm{NH}_{3}\right]$ [SD] $\cdot \mathrm{H}_{2} \mathrm{O}$, Fig 11 . The dimeric $R_{2}{ }^{2}(8)$ hydrogen bonded motif does not occur in this structure. It is replaced by a tertrameric unit comprising the amide $\mathrm{N}$ atom and one pyrimidine ring $\mathrm{N}$ atom of two SD anions interacting with a centrosymmetric dimer of $\mathrm{HOCH}_{2} \mathrm{CH}_{2} \mathrm{NH}_{3}$ cations, see Fig 12 . Thus there are two $R_{3}{ }^{3}(8)$ motifs supported by a central $R_{2}{ }^{2}(10)$ motif. The tetramers are linked by hydrogen bonding through a bifurcated bond from $\mathrm{H} 4 \mathrm{~N}$ to $\mathrm{O}$ and $\mathrm{N}$ acceptors on a SD anion and through the water molecules. Each water molecule interacts with 4 neighbouring SD anions. Thus each water molecule donates two hydrogen bonds to $\mathrm{O}$ atoms of SD anions and accepting two hydrogen bonds from the SD amine groups, see Table 11 for details.

\section{Synthesis and crystallization}

The simple salt forms were prepared by reacting 1:1 molar mixtures of sulfadiazine and $\mathrm{MOH}(M=\mathrm{Li}, \mathrm{Na}, \mathrm{K})$ or the organic base in 50:50 water:ethanol. The mixtures were stirred and heated to give clear solutions before being left to cool to room temperature. Partial evaporation of these reaction mixtures over 4 to 7 days gave suitable crystals of (I), (III), (V) and (VI) but a fine powder for the Na salt. Good quality crystals of the Na salt (II) were obtained by vapour diffusion of ethanol into an aqueous solution of sodium sulfadiazine. The Na OG complex (IV) was obtained by dissolving $0.20 \mathrm{~g}$, $0.44 \mathrm{mmol}$ of NaOG in the minimum amount of water. A slight excess of sulfadiazine $(0.12 \mathrm{~g}, 0.48 \mathrm{mmol})$ was also dissolved in the minimum amount of water. The two solutions were mixed together with stirring and acidified with concentrated $\mathrm{HCl}$. After 3 days, orange crystals of (IV) had grown.

\section{Refinement}

For all structures, $\mathrm{H}$ atoms bound to $\mathrm{C}$ atoms were placed in the expected geometric positions and treated in riding modes with $\mathrm{U}(\mathrm{H})_{\text {iso }}=1.2 \mathrm{U}(\mathrm{C})_{\text {eq. }}$. For aromatic groups $\mathrm{C}-\mathrm{H}=0.95 \AA$ and for $\mathrm{CH}_{2}$ groups $\mathrm{C}-\mathrm{H}=0.95 \AA$. All $\mathrm{H}$ atoms bound to $\mathrm{N}$ were refined freely and isotropically as were $\mathrm{H}$ atoms bound to $\mathrm{O}$ in structure (VI). $\mathrm{H}$ atoms of water molecules in (I), (III) and (IV) were located by difference syntheses and required restraints to be applied such that $\mathrm{O}-\mathrm{H}=0.88(1) \AA$ and $\mathrm{H} \cdots \mathrm{H}=1.33$ (2) $\AA$. For these atoms of (III) $\mathrm{U}(\mathrm{H})_{\text {iso }}=1.5 \mathrm{U}(\mathrm{O})_{\mathrm{eq}}$, whilst for (I) and (IV) U(H) $)_{\text {iso }}$ were refined. 
143

144

fig1.tif

For (IV), after several trial calculations, the three non-coordinated water molecules were given
of 0.5 . For (III) the H atoms of one water ligand were modelled as disordered over three sites.

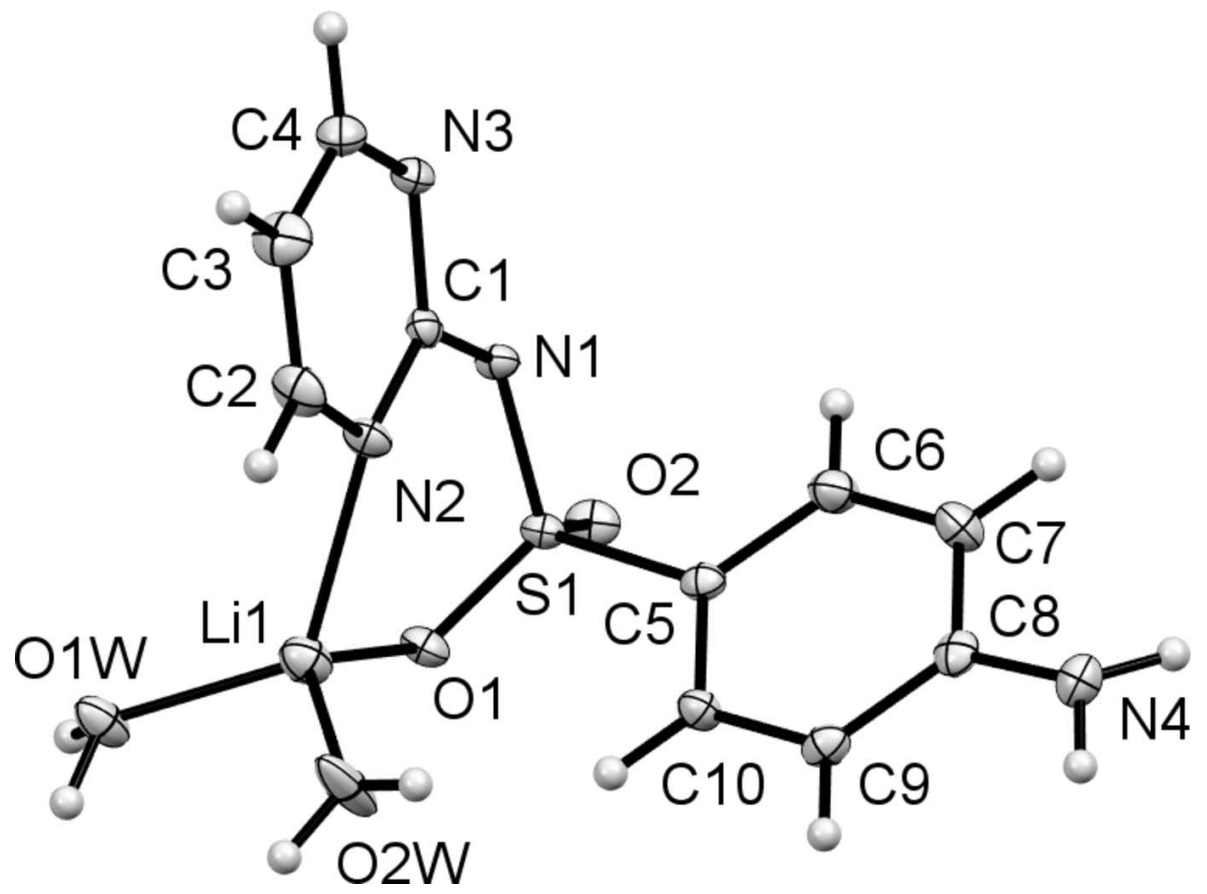

145 Figure 1

146 Molecular structure of the Li salt (I) with non-H atoms shown as 50\% probability ellipsoids. 


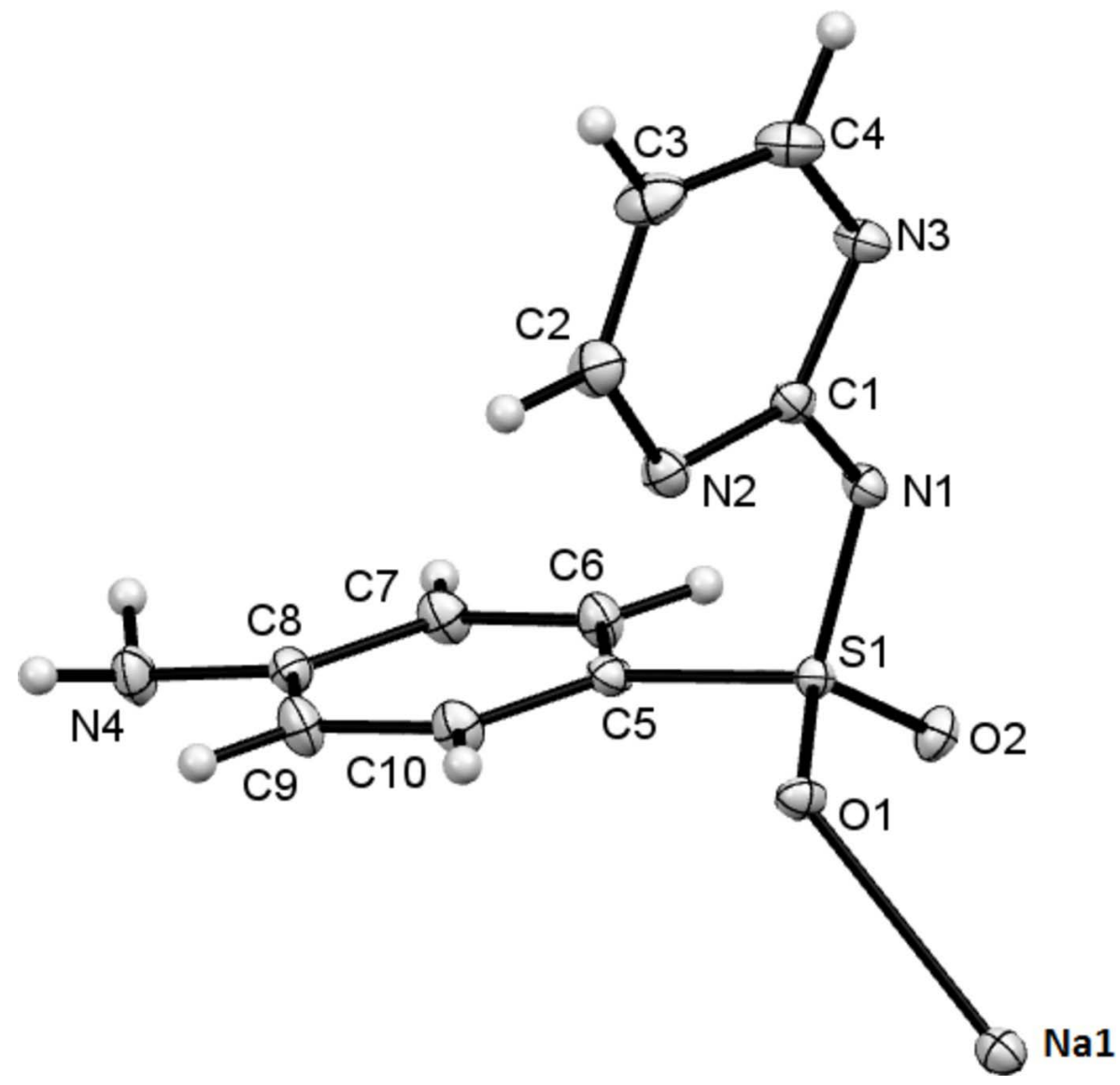

Figure 2

Contents of the asymmetric unit of the Na salt (II) with non-H atoms shown as $50 \%$ probability ellipsoids. 


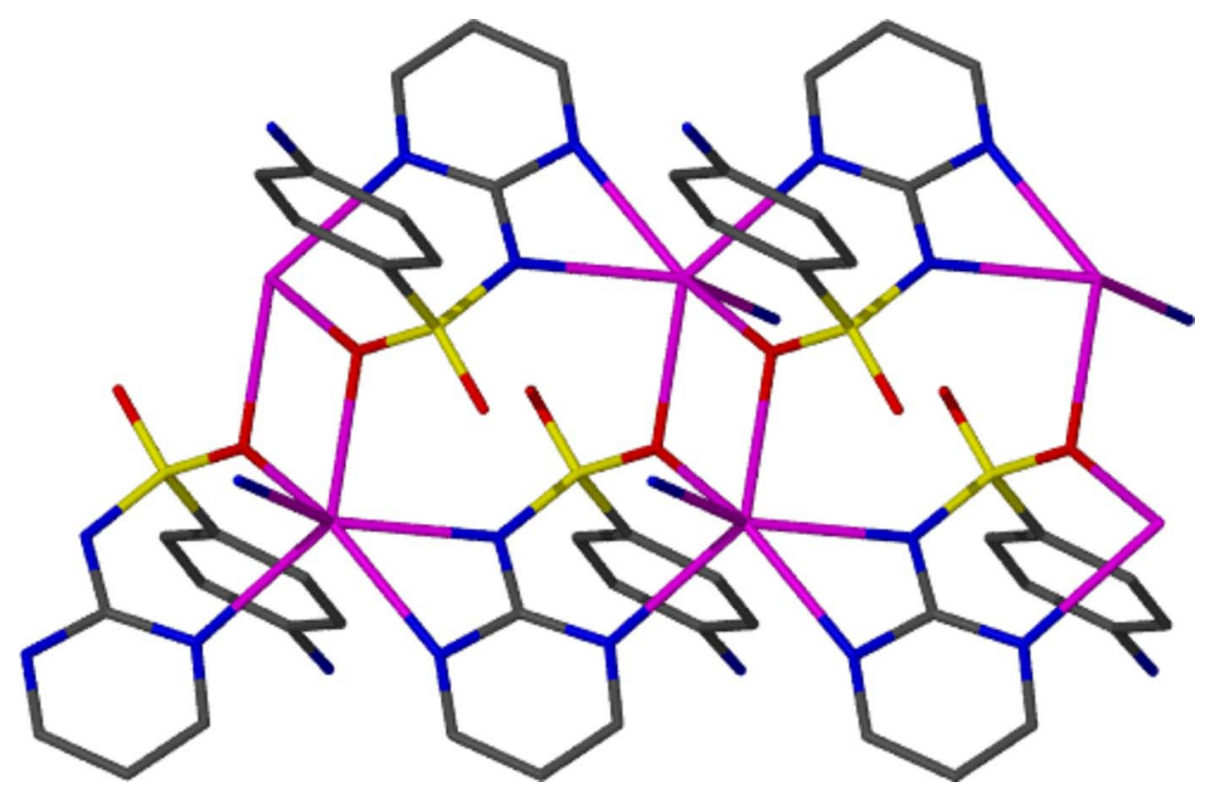

149 Figure 3

Part of the extended structure of (II) showing the dative bonds that give a one-dimensional chain that extends in the crystallographic $a$ direction. $\mathrm{H}$ atoms are omitted for clarity. Here and in other colour figures, black $=\mathrm{C}$, blue $=\mathrm{N}$, red $=$ $\mathrm{O}$, yellow $=\mathrm{S}$, pink $=$ alkali metal.

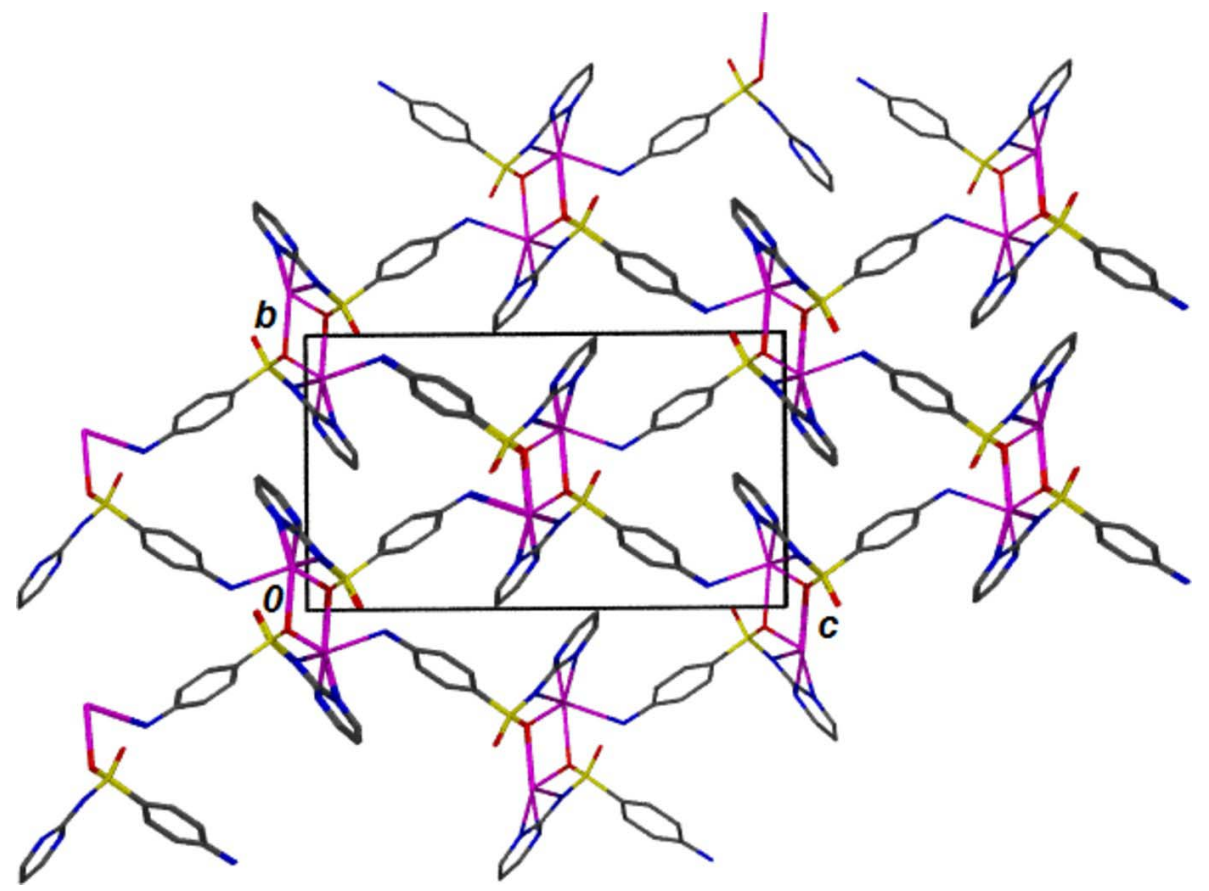

Figure 4

Packing diagram for (II) with view down the $a$ axis direction. 


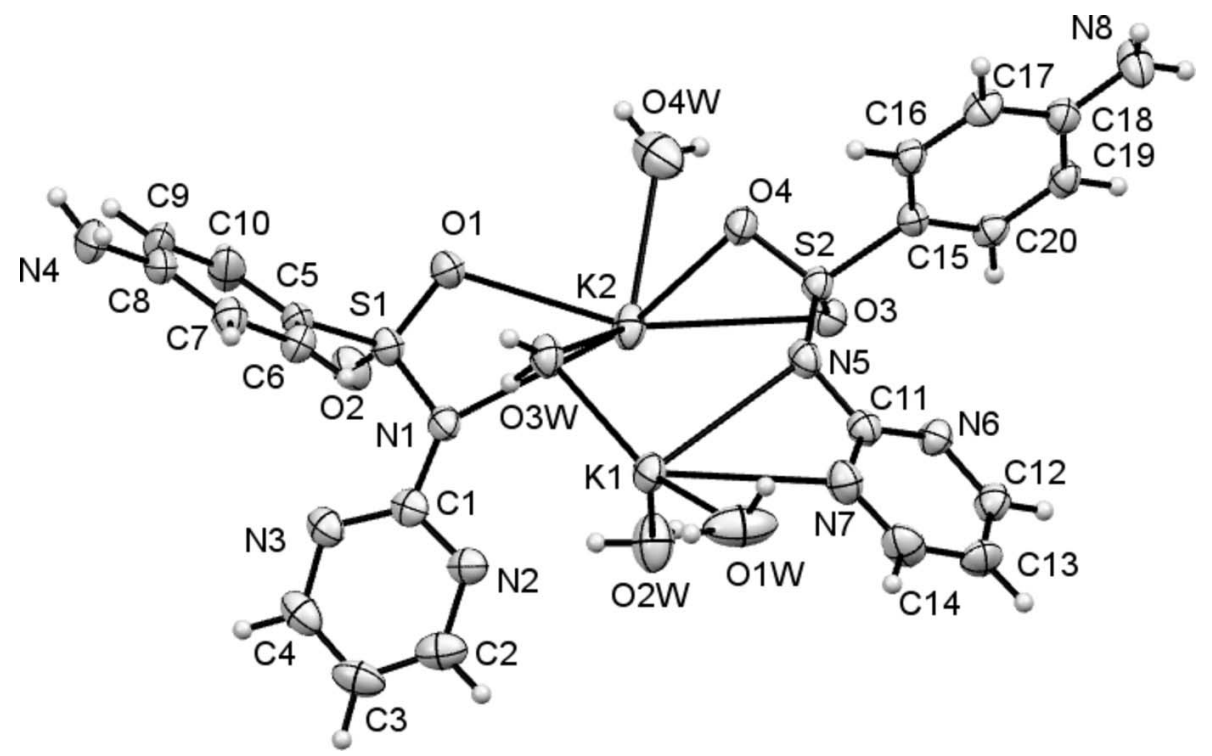

155 Figure 5

156 Contents of the asymmetric unit of the $\mathrm{K}$ salt (III) with non-H atoms shown as $50 \%$ probability ellipsoids. Disordered $\mathrm{H}$ 157 atom positions on water ligand $\mathrm{O} 4 \mathrm{~W}$ are not shown. 


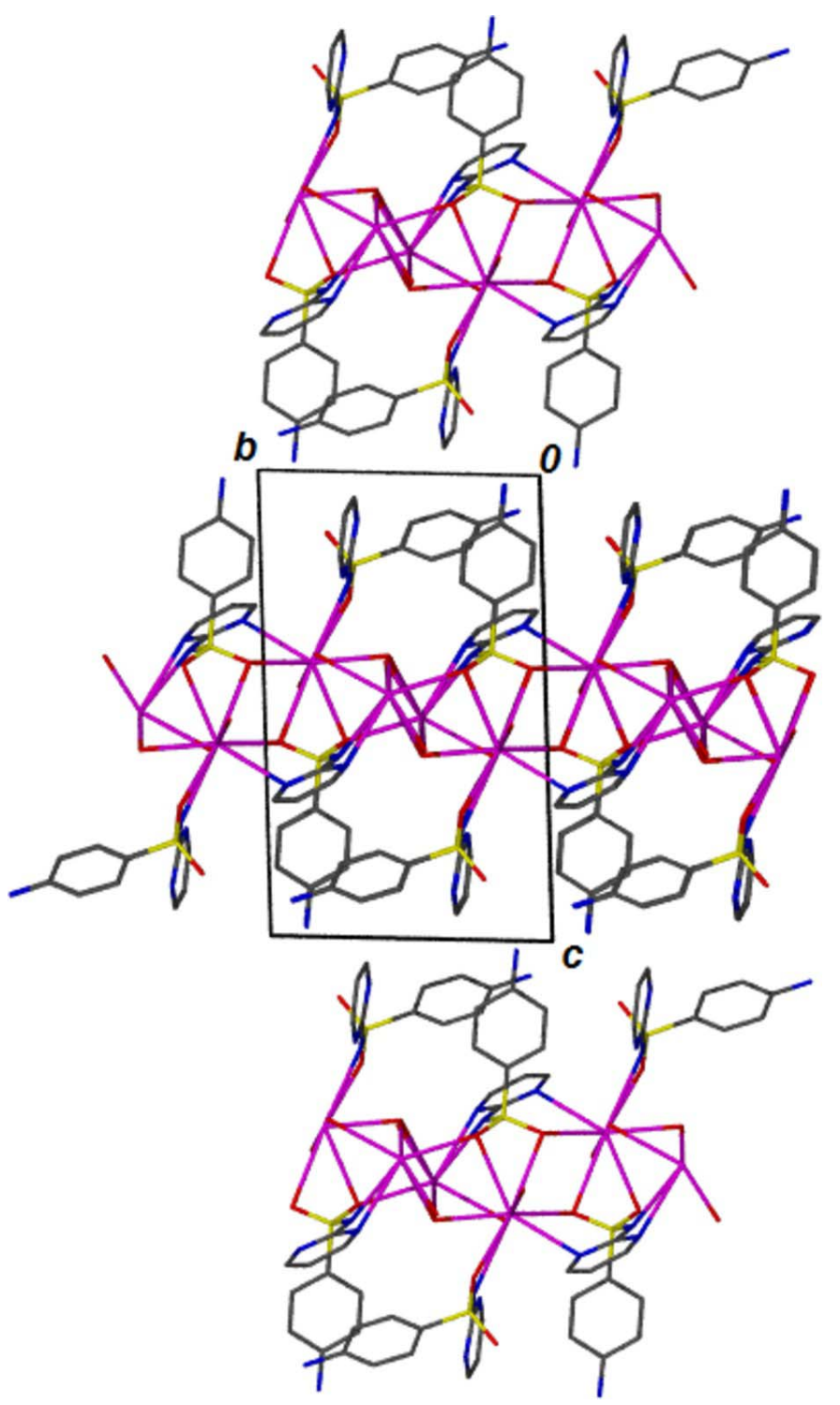

158 Figure 6

159 Packing diagram for showing the layered structure of (III) with view down the $a$ axis direction. 


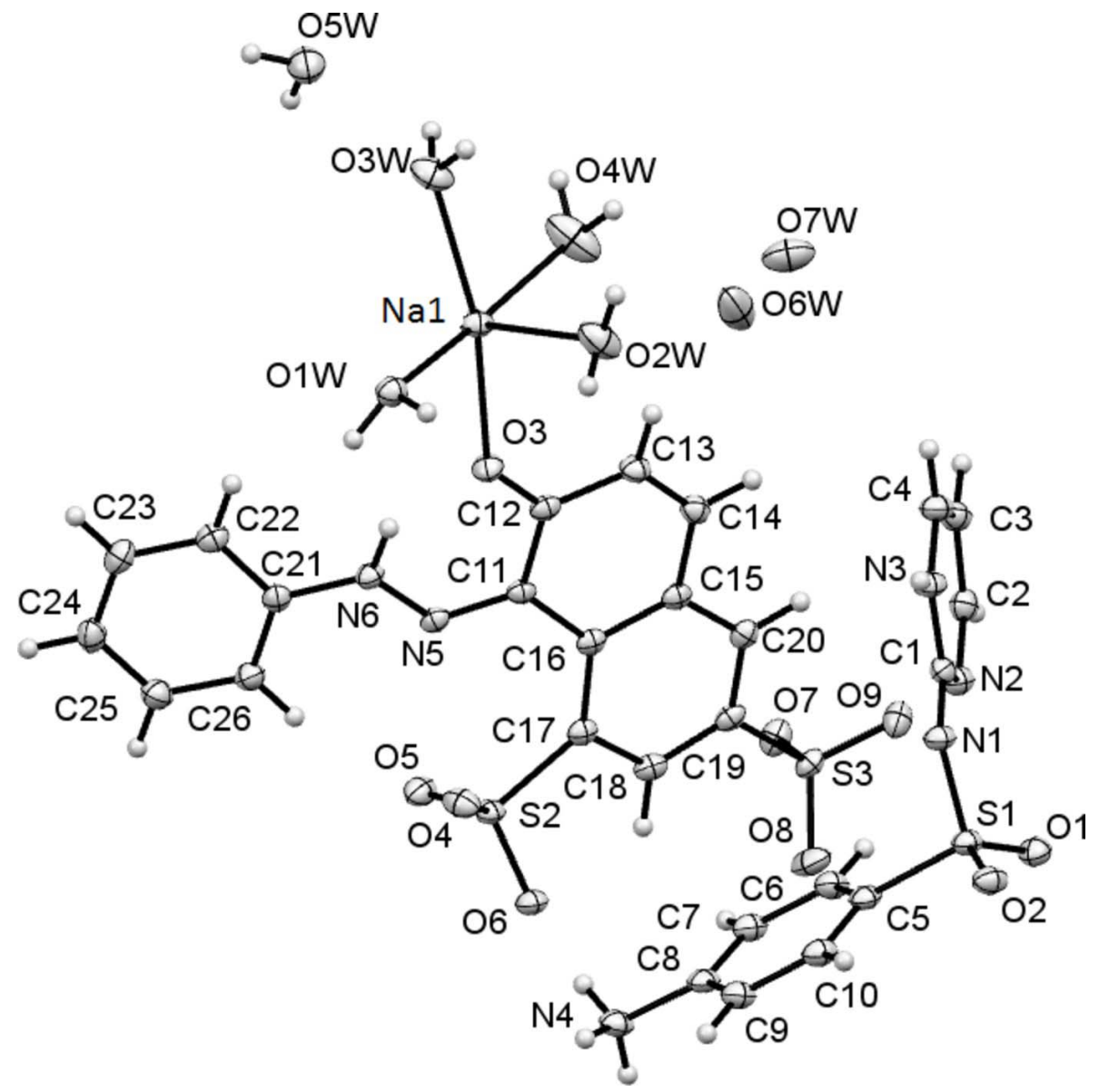

160 Figure 7

Contents of the asymmetric unit of (IV) with non-H atoms shown as 50\% probability ellipsoids. Disordered $\mathrm{H}$ atom positions on water molecules are not shown.

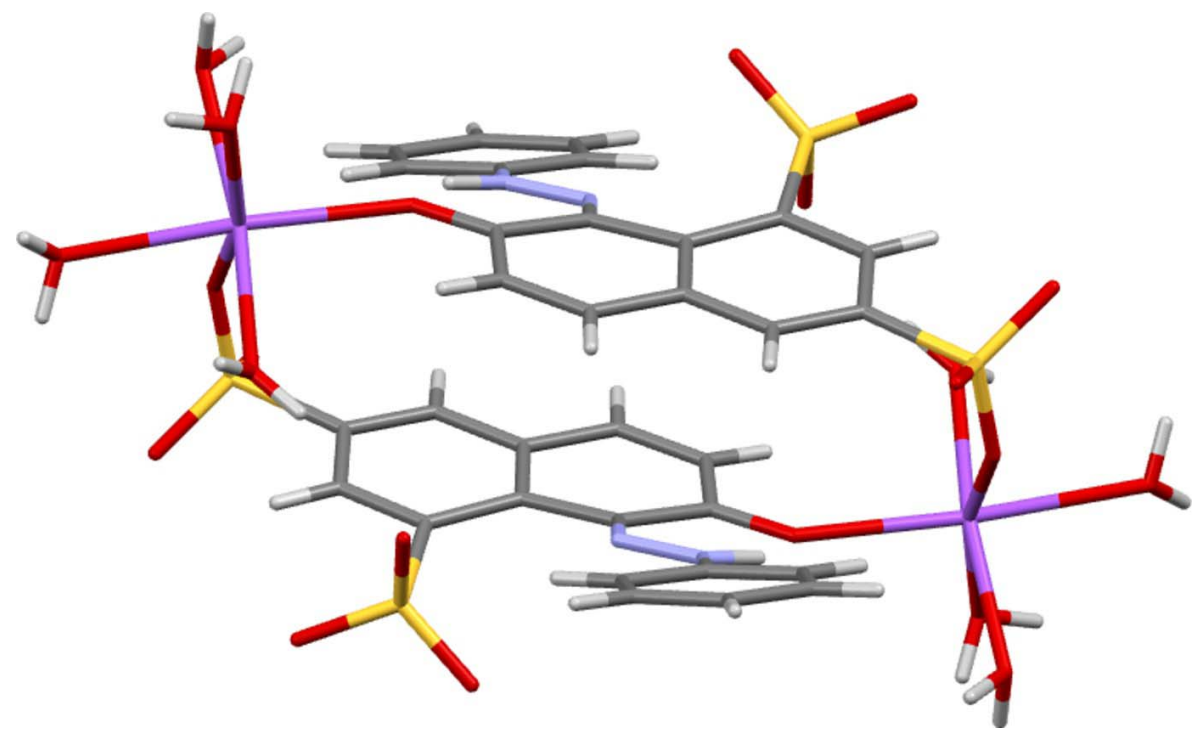


163

164

fig9.tif

165

166

fig10.tif

\section{Figure 8}

View of the dimeric $\left[\mathrm{Na}\left(\mathrm{H}_{2} \mathrm{O}\right)_{4}\right]_{2}[\mathrm{OG}]_{2}$ fragment found in structure (IV).

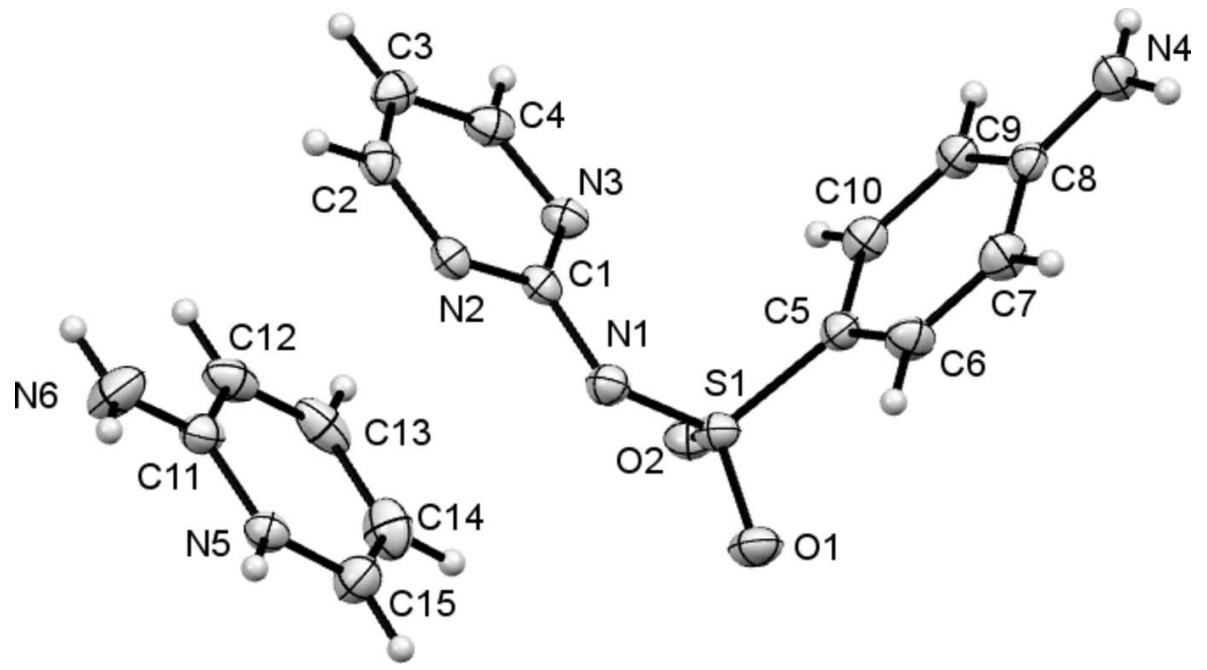

Figure 9

Contents of the asymmetric unit of (V) with non-H atoms shown as 50\% probability ellipsoids.

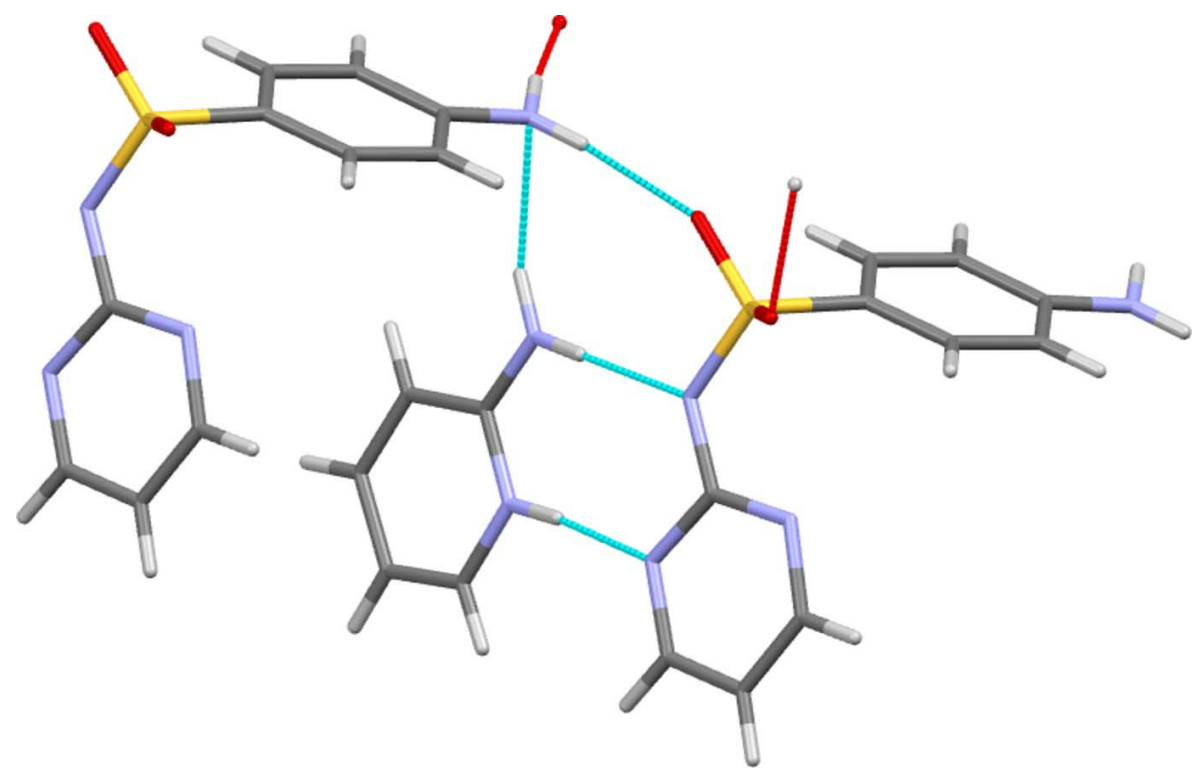

Figure 10

Detail from the structure of $(\mathrm{V})$ highlighting the $R_{2}{ }^{2}(8)$ cation to anion hydrogen bond interaction and the role of the amine groups. 

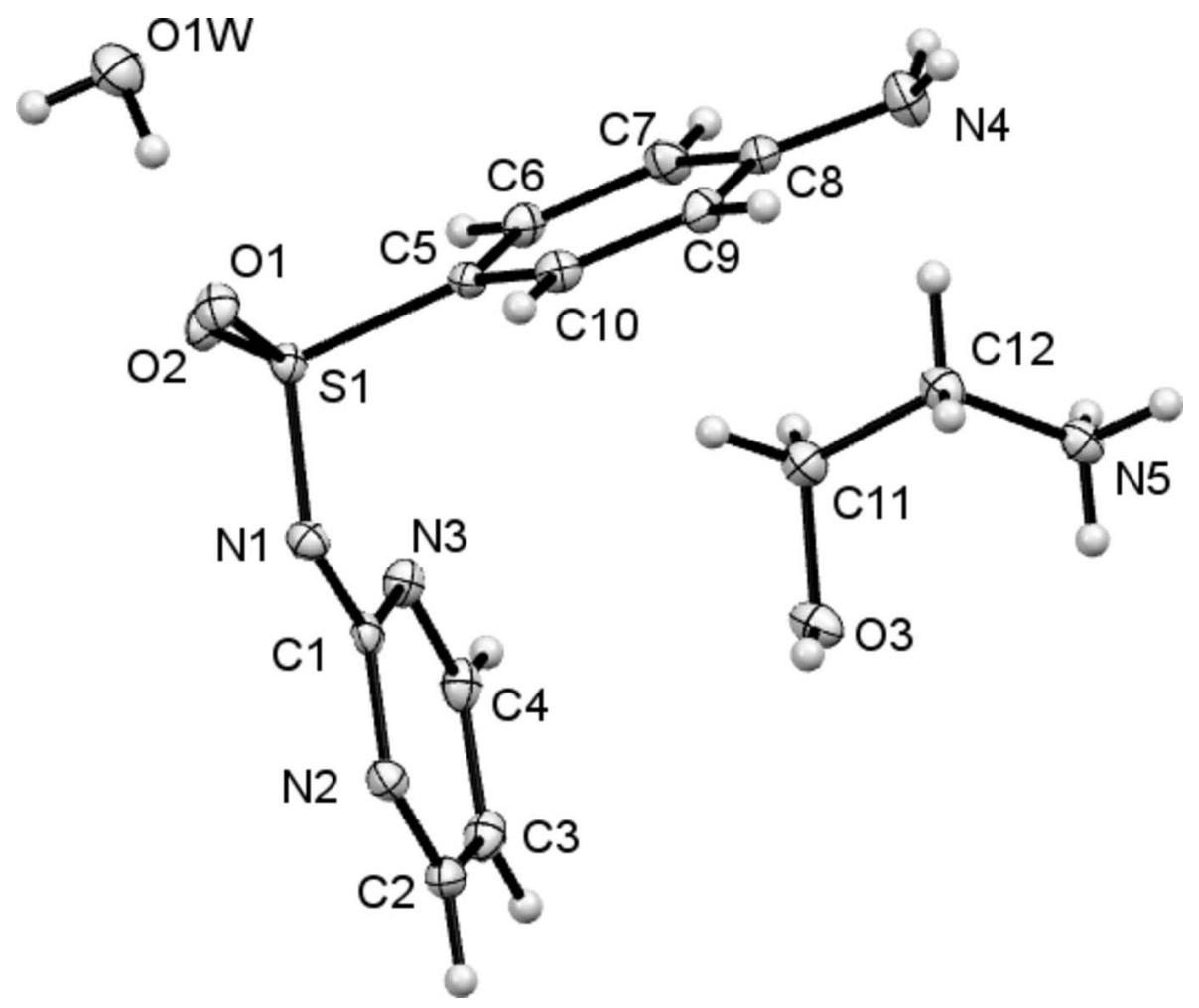

Figure 11

Contents of the asymmetric unit of (VI) with non-H atoms shown as $50 \%$ probability ellipsoids.

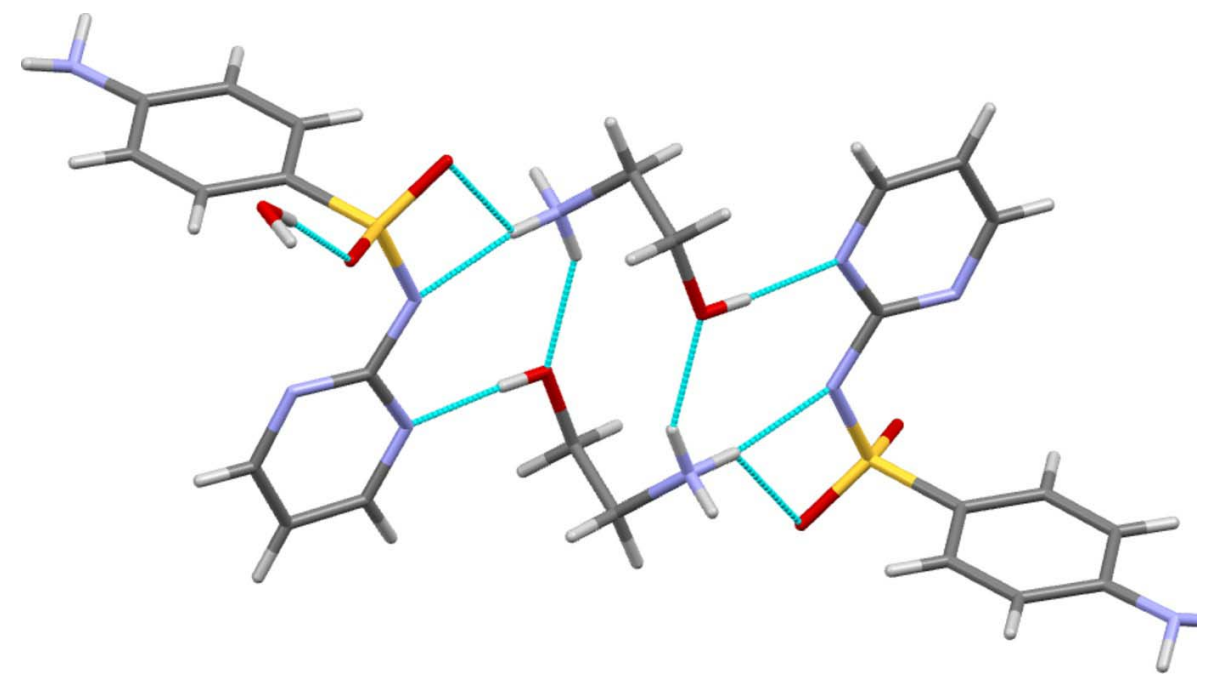

Figure 12

Detail from the structure of (VI) showing the hydrogen bonded tetramer formed by two cations and two anions.

Table 1

Experimental details

(I)

(II)

(III)

(IV)

Crystal data 


\section{$\mathrm{C}_{10} \mathrm{H}_{13} \mathrm{LiN}_{4} \mathrm{O}_{4} \mathrm{~S}$}

292.24

Crystal system, space Monoclinic, $C c$ group

Temperature (K)

$a, b, c(\AA)$

$\alpha, \beta, \gamma\left({ }^{\circ}\right)$

$V\left(\AA^{3}\right)$

Z

Radiation type

$\mu\left(\mathrm{mm}^{-1}\right)$

Crystal size (mm)

Data collection

Diffractometer

Absorption correction

123

(5), 9.5184 (4)

1323.03 (9)

4

Mo $K \alpha$

0.26 Xcalibur E

Multi-scan
11.5095 (4), 12.0788

90, 91.063 (3), 90

$0.30 \times 0.20 \times 0.18$

Oxford Diffraction

CrysAlis PRO, Oxford

Diffraction Ltd.,

Version 1.171.34.40

(release 27-08-2010

CrysAlis171 .NET)

(compiled Aug 27

2010,11:50:40)

Empirical absorption correction using spherical harmonics, implemented in SCALE3 ABSPACK scaling algorithm.

$T_{\min }, T_{\max }$

$0.949,1.000$

$3390,2246,2184$

No. of measured, independent and observed $[I>2 \sigma(I)]$ reflections

$R_{\text {int }}$

$(\sin \theta / \lambda)_{\max }\left(\AA^{-1}\right) \quad 0.682$

$R\left[F^{2}>2 \sigma\left(F^{2}\right)\right]$, $w R\left(F^{2}\right), S$

No. of reflections

No. of parameters

No. of restraints

$\mathrm{H}$-atom treatment

$\Delta \rho_{\max }, \Delta \rho_{\min }\left(\mathrm{e} \AA^{-3}\right)$ and constrained

refinement

$0.28,-0.25$
0.016
0.682

$0.026,0.066,1.04$

2246
207
8

0.029

0.693

0.050

0.623

0.027

0.602

$0.038,0.088,1.06$

$0.060,0.157,1.05$

$0.028,0.075,1.05$

2786

5357

5913

$171 \quad 404$

537

14
$\mathrm{C}_{26} \mathrm{H}_{32} \mathrm{~N}_{6} \mathrm{NaO}_{14.50} \mathrm{~S}_{3}$

779.74

Monoclinic, $P 2_{1} / c$

100

$3238.42(4)$

4

$\mathrm{Cu} K \alpha$

2.95

$0.12 \times 0.08 \times 0.04$

$\mathrm{H}$ atoms treated by a $\mathrm{H}$ atoms treated by a $\mathrm{H}$ atoms treated by a $\mathrm{H}$ atoms treated by a mixture of independent mixture of independent mixture of independent mixture of independent

and constrained

refinement

$0.43,-0.46$ and constrained

refinement

$0.81,-0.45$ and constrained refinement

$0.26,-0.39$ 

No. of measured, independent and observed $[I>2 \sigma(I)]$ reflections

$R_{\text {int }}$ $(\sin \theta / \lambda)_{\max }\left(\AA^{-1}\right)$

Refinement $R\left[F^{2}>2 \sigma\left(F^{2}\right)\right], w R\left(F^{2}\right), S$ No. of reflections No. of parameters No. of restraints $\mathrm{H}$-atom treatment

$\Delta \rho_{\max }, \Delta \rho_{\min }\left(\mathrm{e} \AA^{-3}\right)$ Absolute structure

Crystal data

Temperature $(\mathrm{K})$
(V)

\section{$\mathrm{C}_{15} \mathrm{H}_{16} \mathrm{~N}_{6} \mathrm{O}_{2} \mathrm{~S}$}

344.40

Refined as an inversion?

Win.

$-0.01(8) \quad ?$
?

$?$

?

Crystal system, space group Monoclinic, $P 2_{1} / c$

123

8.5796 (2), $19.0371(5), 11.2512$ (4)

$90,121.116(3), 90$

$1573.27(9)$

4

Mo $K \alpha$

0.23

$0.4 \times 0.3 \times 0.02$

Oxford Diffraction Xcalibur E

Multi-scan

CrysAlis PRO, Oxford Diffraction Ltd., Version 1.171.34.40 (release 27-08-2010 CrysAlis171 .NET) (compiled Aug 27 2010,11:50:40) Empirical absorption correction using spherical harmonics, implemented in SCALE3 ABSPACK scaling algorithm.

$0.527,1.000$

22193, 4082, 3225

0.040

0.682

$0.045,0.114,1.04$

4082

237

0

$\mathrm{H}$ atoms treated by a mixture of independent and constrained refinement

$0.39,-0.48$

?
0.023

(VI)

$\mathrm{C}_{12} \mathrm{H}_{19} \mathrm{~N}_{5} \mathrm{O}_{4} \mathrm{~S}$

329.38

Monoclinic, $P 2{ }_{1} / c$

123

12.7755 (4), 9.8979 (4), 11.5366 (4)

90, $93.508(3), 90$

$1456.08(9)$

4

Mo $K \alpha$

0.25

$0.25 \times 0.24 \times 0.12$

Oxford Diffraction Xcalibur E

Multi-scan

CrysAlis PRO, Oxford Diffraction Ltd., Version 1.171.34.40 (release 27-08-2010

CrysAlis171 .NET) (compiled Aug 27

2010,11:50:40) Empirical absorption correction using spherical harmonics, implemented in SCALE3 ABSPACK scaling algorithm.

$0.904,1.000$

$7213,3571,2942$

0.694

$0.037,0.095,1.06$

3571

231

0

$\mathrm{H}$ atoms treated by a mixture of independent and constrained refinement

$0.45,-0.47$

? 
Absolute structure parameter?

Computer programs: CrysAlis PRO (Agilent, 2014), CrysAlis PRO, SIR92 (Altomare et al., 1994), SHELXS (Sheldrick, 2015), SHELXL2014 (Sheldrick, 2015), Mercury (Macrae et al., 2008), SHELXL2014.

Table 2

Selected geometric parameters $\left(\AA,{ }^{\circ}\right)$ for (I)

\begin{tabular}{llll}
\hline Li1-O2W & $1.870(4)$ & Li1-O1 & $1.934(5)$ \\
Li1-O1W & $1.910(5)$ & Li1-N2 & $2.077(5)$ \\
& & & \\
O2W-Li1-O1W & $101.5(2)$ & O2W-Li1-N2 & $107.1(2)$ \\
O2W-Li1-O1 & $126.9(2)$ & O1W-Li1-N2 & $124.1(2)$ \\
O1W-Li1-O1 & $108.5(2)$ & O1-Li1-N2 & $90.91(18)$ \\
\hline
\end{tabular}

Table 3

Hydrogen-bond geometry $\left(\AA,{ }^{\circ}\right)$ for (I)

\begin{tabular}{lllll}
\hline$D-\mathrm{H} \cdots A$ & $D-\mathrm{H}$ & $\mathrm{H} \cdots A$ & $D \cdots A$ & $D-\mathrm{H} \cdots A$ \\
\hline $\mathrm{O} 1 \mathrm{~W}-\mathrm{H} 1 \mathrm{~W} \cdots \mathrm{O} 2^{\mathrm{i}}$ & $0.875(12)$ & $2.051(16)$ & $2.881(2)$ & $158(3)$ \\
$\mathrm{O} 2 \mathrm{~W}-\mathrm{H} 3 \mathrm{~W} \cdots \mathrm{N} 1^{\mathrm{ii}}$ & $0.865(12)$ & $1.949(15)$ & $2.805(3)$ & $170(4)$ \\
$\mathrm{O} 1 \mathrm{~W}-\mathrm{H} 2 \mathrm{~W} \cdots \mathrm{N} 3^{\mathrm{ii}}$ & $0.867(12)$ & $1.970(12)$ & $2.837(3)$ & $178(3)$ \\
$\mathrm{O} 2 \mathrm{~W}-\mathrm{H} 4 \mathrm{~W} \cdots \mathrm{N} 4^{\mathrm{iii}}$ & $0.863(12)$ & $2.006(12)$ & $2.866(3)$ & $174(4)$ \\
$\mathrm{N} 4-\mathrm{H} 1 \mathrm{~N} \cdots \mathrm{O} 2^{\text {iv }}$ & $0.84(3)$ & $2.40(3)$ & $3.070(3)$ & $137(3)$
\end{tabular}

Symmetry codes: (i) $x,-y+1, z-1 / 2$; (ii) $x+1 / 2,-y+1 / 2, z-1 / 2$; (iii) $x,-y, z-1 / 2$; (iv) $x+1 / 2, y-1 / 2, z$.

Table 4

Selected bond lengths ( $\AA$ ) for (II)

\begin{tabular}{llll}
\hline $\mathrm{Na} 1-\mathrm{O} 1^{\mathrm{i}}$ & $2.2897(15)$ & $\mathrm{Na} 1-\mathrm{N} 3^{\mathrm{ii}}$ & $2.4836(18)$ \\
$\mathrm{Na} 1-\mathrm{N} 1^{\mathrm{ii}}$ & $2.4533(15)$ & $\mathrm{Na} 1-\mathrm{N} 4^{\mathrm{iii}}$ & $2.5771(19)$ \\
$\mathrm{Na} 1-\mathrm{O} 1$ & $2.4647(17)$ & $\mathrm{Na} 1-\mathrm{N} 2^{\mathrm{i}}$ & $2.6670(17)$
\end{tabular}

Symmetry codes: (i) $-x+1,-y,-z$; (ii) $-x,-y,-z$; (iii) $-x+1, y-1 / 2,-z+1 / 2$.

Table 5

Hydrogen-bond geometry $\left(\AA,{ }^{\circ}\right)$ for (II)

\begin{tabular}{lllll}
\hline$D-\mathrm{H} \cdots A$ & $D-\mathrm{H}$ & $\mathrm{H} \cdots A$ & $D \cdots A$ & $D-\mathrm{H} \cdots A$ \\
\hline $\mathrm{N} 4-\mathrm{H} 1 \mathrm{~N} \cdots \mathrm{O} 2^{\text {iv }}$ & $0.86(2)$ & $2.06(2)$ & $2.907(2)$ & $166(2)$ \\
$\mathrm{N} 4-\mathrm{H} 2 \mathrm{~N} \cdots \mathrm{O}^{\mathrm{v}}$ & $0.91(2)$ & $2.52(2)$ & $2.959(2)$ & $110.5(17)$
\end{tabular}

Symmetry codes: (iv) $-x, y+1 / 2,-z+1 / 2 ;$ (v) $-x+1, y+1 / 2,-z+1 / 2$.

Table 6

Selected bond lengths ( $\AA$ ) for (III)

\begin{tabular}{llll}
\hline $\mathrm{K} 1-\mathrm{O} 4^{\mathrm{i}}$ & $2.729(3)$ & $\mathrm{K} 2-\mathrm{O}^{\mathrm{iii}}$ & $2.759(3)$ \\
$\mathrm{K} 1-\mathrm{O} 3 \mathrm{~W}$ & $2.771(3)$ & $\mathrm{K} 2-\mathrm{O} 4 \mathrm{~W}$ & $2.820(4)$
\end{tabular}



$\mathrm{K} 1-\mathrm{O} 1 \mathrm{~W}^{\mathrm{ii}}$
$\mathrm{K} 1-\mathrm{O} 2 \mathrm{~W}$
$\mathrm{K} 1-\mathrm{N} 7$
$\mathrm{K} 1-\mathrm{O} 1 \mathrm{~W}$
K1-O3W ${ }^{\mathrm{i}}$
K1-N5

$\mathrm{K} 2-\mathrm{O} 3 \mathrm{~W}$
$\mathrm{~K} 2-\mathrm{O} 1$
$\mathrm{~K} 2-\mathrm{N}^{\mathrm{iii}}$
$\mathrm{K} 2-\mathrm{O} 4$
$\mathrm{~K} 2-\mathrm{O} 3$
$\mathrm{~K} 2-\mathrm{N} 1$

$2.871(3)$

2.883 (3)

2.899 (4)

2.947 (3)

3.023 (3)

3.153 (3)

Symmetry codes: (i) $-x,-y+1,-z+1$; (ii) $-x+1,-y+1,-z+1$; (iii) $-x,-y+2,-z+1$.

Table 7

Hydrogen-bond geometry $\left(\AA,{ }^{\circ}\right)$ for (III)

\begin{tabular}{lllll}
\hline$D-\mathrm{H} \cdots A$ & $D-\mathrm{H}$ & $\mathrm{H} \cdots A$ & $D \cdots A$ & $D-\mathrm{H} \cdots A$ \\
\hline $\mathrm{O} 1 \mathrm{~W}-\mathrm{H} 1 \mathrm{~W} \cdots \mathrm{O} 1^{\mathrm{i}}$ & $0.880(10)$ & $1.93(2)$ & $2.799(5)$ & $167(8)$ \\
$\mathrm{O} 1 \mathrm{~W}-\mathrm{H} 2 \mathrm{~W} \cdots \mathrm{O}^{\mathrm{N}} \mathrm{W}^{\mathrm{i}}$ & $0.877(10)$ & $2.27(4)$ & $3.070(6)$ & $151(6)$ \\
$\mathrm{O} 2 \mathrm{~W}-\mathrm{H} 3 \mathrm{~W} \cdots 3^{\mathrm{iii}}$ & $0.878(10)$ & $2.071(19)$ & $2.933(5)$ & $167(6)$ \\
$\mathrm{O} 2 \mathrm{~W}-\mathrm{H} 4 \mathrm{~W} \cdots \mathrm{N} 2$ & $0.880(10)$ & $1.965(17)$ & $2.828(5)$ & $167(6)$ \\
$\mathrm{O} 3 \mathrm{~W}-\mathrm{H} 5 \mathrm{~W} \cdots \mathrm{N} 1$ & $0.874(10)$ & $2.022(18)$ & $2.872(5)$ & $164(5)$ \\
$\mathrm{O} 3 \mathrm{~W}-\mathrm{H} 6 \mathrm{~W} \cdots \mathrm{N} 5^{\mathrm{i}}$ & $0.873(10)$ & $1.979(14)$ & $2.835(5)$ & $166(4)$ \\
$\mathrm{O} 4 \mathrm{~W}-\mathrm{H} 7 \mathrm{~W} \cdots \mathrm{O} 2 \mathrm{~W}^{\mathrm{iv}}$ & $0.878(10)$ & $2.052(17)$ & $2.919(6)$ & $169(7)$ \\
$\mathrm{O} 4 \mathrm{~W}-\mathrm{H} 8 \mathrm{~W} \cdots \mathrm{O} 4 \mathrm{~W}^{\mathrm{v}}$ & $0.879(10)$ & $2.05(2)$ & $2.920(8)$ & $168(9)$ \\
$\mathrm{N} 4-\mathrm{H} 1 \mathrm{~N} \cdots \mathrm{N} 4^{\mathrm{vi}}$ & $0.87(7)$ & $2.50(6)$ & $3.054(9)$ & $122(5)$ \\
$\mathrm{N} 4-\mathrm{H} 2 \mathrm{~N} \cdots 2^{\text {vii }}$ & $0.93(7)$ & $2.57(7)$ & $3.431(7)$ & $153(5)$ \\
$\mathrm{N} 8-\mathrm{H} 3 \mathrm{~N} \cdots \mathrm{O} 2^{\text {viii }}$ & $0.87(5)$ & $2.38(5)$ & $3.046(5)$ & $134(4)$ \\
$\mathrm{N} 8-\mathrm{H} 3 \mathrm{~N} \cdots \mathrm{N} 3^{\text {viii }}$ & $0.87(5)$ & $2.61(5)$ & $3.283(6)$ & $135(4)$ \\
& & & &
\end{tabular}

Symmetry codes: (i) $-x,-y+1,-z+1$; (iii) $-x,-y+2,-z+1$; (iv) $x-1, y, z$; (v) $-x-1,-y+2,-z+1$; (vi) $-x-1,-y,-z$; (vii) $x, y-1, z$; (viii) $x, y, z+1$.

Table 8

Selected geometric parameters $\left(\AA,^{\circ}\right)$ for (IV)

\begin{tabular}{llll}
\hline $\mathrm{Na} 1-\mathrm{O} 1 \mathrm{~W}$ & $2.3184(13)$ & $\mathrm{Na} 1-\mathrm{O} 2 \mathrm{~W}$ & $2.4764(14)$ \\
$\mathrm{Na} 1-\mathrm{O} 4 \mathrm{~W}$ & $2.3211(15)$ & $\mathrm{O} 3-\mathrm{C} 12$ & $1.2626(18)$ \\
$\mathrm{Na} 1-\mathrm{O} 3$ & $2.4042(12)$ & $\mathrm{N} 5-\mathrm{N} 6$ & $1.3031(18)$ \\
$\mathrm{Na} 1-\mathrm{O} 7^{\mathrm{i}}$ & $2.4237(12)$ & $\mathrm{N} 5-\mathrm{C} 11$ & $1.3322(19)$ \\
$\mathrm{Na} 1-\mathrm{O} 3 \mathrm{~W}$ & $2.4592(14)$ & $\mathrm{C} 11-\mathrm{C} 12$ & $1.467(2)$ \\
& & & \\
$\mathrm{O} 1 \mathrm{~W}-\mathrm{Na} 1-\mathrm{O} 4 \mathrm{~W}$ & $169.11(5)$ & $\mathrm{O} 3-\mathrm{Na} 1-\mathrm{O} 3 \mathrm{~W}$ & $166.97(5)$ \\
$\mathrm{O} 1 \mathrm{~W}-\mathrm{Na} 1-\mathrm{O} 3$ & $90.96(4)$ & $\mathrm{O} 7-\mathrm{Na} 1-\mathrm{O} 3 \mathrm{~W}$ & $80.17(5)$ \\
$\mathrm{O} 4 \mathrm{~W}-\mathrm{Na} 1-\mathrm{O} 3$ & $98.12(5)$ & $\mathrm{O} 1 \mathrm{~W}-\mathrm{Na} 1-\mathrm{O} 2 \mathrm{~W}$ & $81.14(5)$ \\
$\mathrm{O} 1 \mathrm{~W}-\mathrm{Na} 1-\mathrm{O} 7^{\mathrm{i}}$ & $85.13(4)$ & $\mathrm{O} 4 \mathrm{~W}-\mathrm{Na} 1-\mathrm{O} 2 \mathrm{~W}$ & $91.10(6)$ \\
$\mathrm{O} 4 \mathrm{~W}-\mathrm{Na} 1-\mathrm{O} 7^{\mathrm{i}}$ & $101.30(5)$ & $\mathrm{O} 3-\mathrm{Na} 1-\mathrm{O} 2 \mathrm{~W}$ & $101.64(5)$ \\
$\mathrm{O} 3-\mathrm{Na} 1-\mathrm{O} 7^{\mathrm{i}}$ & $86.81(4)$ & $\mathrm{O} 7-\mathrm{Na} 1-\mathrm{O} 2 \mathrm{~W}$ & $163.94(5)$ \\
$\mathrm{O} 1 \mathrm{~W}-\mathrm{Na} 1-\mathrm{O} 3 \mathrm{~W}$ & $87.73(5)$ & $\mathrm{O} 3 \mathrm{~W}-\mathrm{Na} 1-\mathrm{O} 2 \mathrm{~W}$ & $90.97(5)$ \\
$\mathrm{O} 4 \mathrm{~W}-\mathrm{Na} 1-\mathrm{O} 3 \mathrm{~W}$ & $84.75(6)$ & &
\end{tabular}

Symmetry code: (i) $-x,-y+1,-z$. 
Table 9

Hydrogen-bond geometry $\left(\AA,^{\circ}\right)$ for (IV)

\begin{tabular}{|c|c|c|c|c|}
\hline$D-\mathrm{H} \cdots A$ & $D-\mathrm{H}$ & $\mathrm{H} \cdots A$ & $D \cdots A$ & $D-\mathrm{H} \cdots A$ \\
\hline $\mathrm{O} 1 \mathrm{~W}-\mathrm{H} 1 \mathrm{~W} \cdots \mathrm{O} 9^{\mathrm{ii}}$ & $0.88(3)$ & $1.99(3)$ & $2.8404(16)$ & $163(2)$ \\
\hline $\mathrm{O} 1 \mathrm{~W}-\mathrm{H} 2 \mathrm{~W} \cdots \mathrm{O} 1^{\mathrm{ii}}$ & $0.91(3)$ & $2.24(3)$ & $2.8830(17)$ & $127(2)$ \\
\hline $\mathrm{O} 1 \mathrm{~W}-\mathrm{H} 2 \mathrm{~W} \cdots \mathrm{N} 2^{\mathrm{ii}}$ & $0.91(3)$ & $2.07(3)$ & $2.8972(18)$ & $150(2)$ \\
\hline $\mathrm{O} 2 \mathrm{~W}-\mathrm{H} 3 \mathrm{~W} \cdots \mathrm{O} 9^{\mathrm{ii}}$ & $0.86(3)$ & $2.05(3)$ & $2.8852(18)$ & $163(2)$ \\
\hline $\mathrm{O} 2 \mathrm{~W}-\mathrm{H} 4 \mathrm{~W} \cdots \mathrm{O} 6^{\mathrm{iii}}$ & $0.92(4)$ & $1.98(4)$ & $2.8932(18)$ & $174(3)$ \\
\hline $\mathrm{O} 3 \mathrm{~W}-\mathrm{H} 5 \mathrm{~W} \cdots \mathrm{O} 5^{\mathrm{iii}}$ & $0.86(3)$ & $1.98(3)$ & $2.8320(18)$ & $178(3)$ \\
\hline $\mathrm{O} 3 \mathrm{~W}-\mathrm{H} 6 \mathrm{~W} \cdots \mathrm{O} 5 \mathrm{~W}$ & $0.83(4)$ & $2.18(3)$ & $2.736(3)$ & $124(3)$ \\
\hline $\mathrm{O} 3 \mathrm{~W}-\mathrm{H} 6 \mathrm{~W} \cdots \mathrm{O} 6 \mathrm{~W}^{\mathrm{iv}}$ & $0.83(4)$ & 2.09 (4) & $2.853(3)$ & $153(3)$ \\
\hline $\mathrm{O} 3 \mathrm{~W}-\mathrm{H} 6 \mathrm{~W} \cdots \mathrm{O} 7 \mathrm{~W}^{\mathrm{iv}}$ & $0.83(4)$ & $2.06(4)$ & $2.799(3)$ & $148(3)$ \\
\hline $\mathrm{O} 4 \mathrm{~W}-\mathrm{H} 7 \mathrm{~W} \cdots \mathrm{O} 5^{\mathrm{i}}$ & $0.879(10)$ & $2.242(11)$ & $3.1093(19)$ & $169(2)$ \\
\hline $\mathrm{O} 4 \mathrm{~W}-\mathrm{H} 8 \mathrm{~W} \cdots \mathrm{O} 4 \mathrm{~W}^{\mathrm{iv}}$ & $0.883(10)$ & $2.00(2)$ & $2.825(3)$ & $154(4)$ \\
\hline $\mathrm{O} 4 \mathrm{~W}-\mathrm{H} 8 \mathrm{~W} \cdots \mathrm{O} 6 \mathrm{~W}$ & $0.883(10)$ & $2.41(5)$ & $2.871(3)$ & $113(4)$ \\
\hline $\mathrm{O} 4 \mathrm{~W}-\mathrm{H} 14 \mathrm{~W} \cdots \mathrm{O} 7 \mathrm{~W}^{\mathrm{iv}}$ & $0.886(10)$ & $2.16(3)$ & $2.902(3)$ & $140(4)$ \\
\hline $\mathrm{O} 5 \mathrm{~W}-\mathrm{H} 9 \mathrm{~W} \cdots 4^{v}$ & $0.883(10)$ & $1.845(13)$ & $2.710(3)$ & $166(4)$ \\
\hline $\mathrm{O} 5 \mathrm{~W}-\mathrm{H} 10 \mathrm{~W} \cdots \mathrm{O}^{\mathrm{i}}$ & $0.885(10)$ & $1.986(14)$ & $2.843(3)$ & $163(3)$ \\
\hline $\mathrm{O} 6 \mathrm{~W}-\mathrm{H} 11 \mathrm{~W} \cdots \mathrm{O} 2^{\mathrm{vi}}$ & $0.886(10)$ & $2.22(2)$ & $2.893(3)$ & $132(2)$ \\
\hline $\mathrm{O} 6 \mathrm{~W}-\mathrm{H} 12 \mathrm{~W} \cdots \mathrm{O} 2 \mathrm{~W}$ & $0.881(10)$ & $1.850(11)$ & $2.731(3)$ & $178(5)$ \\
\hline $\mathrm{O} 7 \mathrm{~W}-\mathrm{H} 13 \mathrm{~W} \cdots \mathrm{O} 8^{\mathrm{iii}}$ & $0.875(10)$ & $1.91(2)$ & $2.699(3)$ & $150(3)$ \\
\hline $\mathrm{N} 6-\mathrm{H} 1 \mathrm{~N} \cdots \mathrm{O} 3$ & $0.92(2)$ & $1.75(2)$ & $2.5342(16)$ & $142(2)$ \\
\hline $\mathrm{N} 4-\mathrm{H} 2 \mathrm{~N} \cdots \mathrm{O} 3 \mathrm{~W}^{\mathrm{vii}}$ & $0.92(3)$ & $1.93(3)$ & $2.839(2)$ & $171(2)$ \\
\hline $\mathrm{N} 4-\mathrm{H} 3 \mathrm{~N} \cdots \mathrm{O} 6$ & $0.91(2)$ & $1.92(2)$ & $2.8245(18)$ & $170(2)$ \\
\hline $\mathrm{N} 4-\mathrm{H} 4 \mathrm{~N} \cdots \mathrm{O} 1^{\text {viii }}$ & $0.91(2)$ & $2.01(2)$ & $2.8784(18)$ & $159.6(19)$ \\
\hline $\mathrm{N} 4-\mathrm{H} 4 \mathrm{~N} \cdots \mathrm{O} 1 \mathrm{~W}^{\mathrm{vii}}$ & $0.91(2)$ & $2.50(2)$ & $2.9386(19)$ & $110.5(16)$ \\
\hline $\mathrm{N} 3-\mathrm{H} 5 \mathrm{~N} \cdots \mathrm{O} 2^{\mathrm{vi}}$ & $0.84(2)$ & $2.57(2)$ & $3.0827(17)$ & $120.3(18)$ \\
\hline $\mathrm{N} 3-\mathrm{H} 5 \mathrm{~N} \cdots \mathrm{N} 1^{\mathrm{vi}}$ & $0.84(2)$ & $2.02(2)$ & $2.8655(19)$ & $177(2)$ \\
\hline
\end{tabular}

Symmetry codes: (i) $-x,-y+1,-z$; (ii) $-x, y-1 / 2,-z+1 / 2$; (iii) $x-1, y, z$; (iv) $-x-1,-y+1,-z$; (v) $x-1,-y+1 / 2, z-1 / 2$; (vi) $-x,-y+1,-z+1$; (vii) $x+1$, $-y+1 / 2, z+1 / 2 ;$ (viii) $-x+1,-y+1,-z+1$.

Table 10

Hydrogen-bond geometry $\left(\AA,{ }^{\circ}\right)$ for $(\mathrm{V})$

\begin{tabular}{lllll}
\hline$D-\mathrm{H} \cdots A$ & $D-\mathrm{H}$ & $\mathrm{H} \cdots A$ & $D \cdots A$ & $D-\mathrm{H} \cdots A$ \\
\hline $\mathrm{N} 4-\mathrm{H} 1 \mathrm{~N} \cdots \mathrm{O} 2^{\mathrm{i}}$ & $0.86(2)$ & $2.04(2)$ & $2.895(2)$ & $168(2)$ \\
$\mathrm{N} 4-\mathrm{H} 2 \mathrm{~N} \cdots \mathrm{O} 1^{i i}$ & $0.87(2)$ & $2.03(2)$ & $2.887(2)$ & $174(2)$ \\
$\mathrm{N} 5-\mathrm{H} 3 \mathrm{~N} \cdots \mathrm{N} 2^{\mathrm{iii}}$ & $0.92(2)$ & $1.85(2)$ & $2.758(2)$ & $174(2)$ \\
$\mathrm{N} 6-\mathrm{H} 4 \mathrm{~N} \cdots \mathrm{N} 4^{\text {iv }}$ & $0.87(3)$ & $2.39(3)$ & $3.085(2)$ & $137(2)$ \\
$\mathrm{N} 6-\mathrm{H} 5 \mathrm{~N} \cdots \mathrm{N} 1^{\mathrm{iii}}$ & $0.92(3)$ & $2.00(3)$ & $2.918(2)$ & $173(3)$ \\
\hline
\end{tabular}

Symmetry codes: (i) $x-1,-y+3 / 2, z-1 / 2$; (ii) $x-1, y, z$; (iii) $-x+2,-y+2,-z$; (iv) $-x+1,-y+2,-z$. 
Table 11

Hydrogen-bond geometry $\left(\AA,^{\circ}\right)$ for (VI)

\begin{tabular}{lllll}
\hline$D-\mathrm{H} \cdots A$ & $D-\mathrm{H}$ & $\mathrm{H} \cdots A$ & $D \cdots A$ & $D-\mathrm{H} \cdots A$ \\
\hline $\mathrm{N} 4-\mathrm{H} 1 \mathrm{~N} \cdots \mathrm{O}^{2} W^{\mathrm{i}}$ & $0.89(2)$ & $2.34(2)$ & $3.173(2)$ & $156(2)$ \\
$\mathrm{N} 4-\mathrm{H} 2 \mathrm{~N} \cdots \mathrm{O}^{\mathrm{ii}}$ & $0.83(2)$ & $2.58(2)$ & $3.293(2)$ & $144.1(19)$ \\
$\mathrm{N} 5-\mathrm{H} 3 \mathrm{~N} \cdots \mathrm{O}^{\mathrm{iii}}$ & $0.86(2)$ & $2.01(2)$ & $2.7762(18)$ & $147.2(18)$ \\
$\mathrm{N} 5-\mathrm{H} 4 \mathrm{~N} \cdots 2^{\mathrm{ii}}$ & $0.90(2)$ & $1.99(2)$ & $2.8488(18)$ & $159(2)$ \\
$\mathrm{N} 5-\mathrm{H} 4 \mathrm{~N} \cdots \mathrm{N} 3^{\mathrm{ii}}$ & $0.90(2)$ & $2.51(2)$ & $3.038(2)$ & $118.1(17)$ \\
$\mathrm{N} 5-\mathrm{H} 5 \mathrm{~N} \cdots 1^{\mathrm{iv}}$ & $0.93(2)$ & $2.44(2)$ & $3.1006(18)$ & $128.4(17)$ \\
$\mathrm{N} 5-\mathrm{H} 5 \mathrm{~N} \cdots \mathrm{N} 1^{\text {iv }}$ & $0.93(2)$ & $2.02(2)$ & $2.933(2)$ & $167.3(19)$ \\
$\mathrm{O} 3-\mathrm{H} 1 \mathrm{H} \cdots \mathrm{N} 2^{\mathrm{v}}$ & $0.84(3)$ & $1.90(3)$ & $2.7399(19)$ & $177(2)$ \\
$\mathrm{O} 1 \mathrm{~W}-\mathrm{H} 1 \mathrm{~W} \cdots \mathrm{O} 2$ & $0.97(3)$ & $1.94(3)$ & $2.9039(17)$ & $174(3)$ \\
$\mathrm{O} 1 \mathrm{~W}-\mathrm{H} 2 \mathrm{~W} \cdots \mathrm{O} 1^{\mathrm{vi}}$ & $0.86(3)$ & $2.07(3)$ & $2.8997(18)$ & $163(2)$
\end{tabular}

Symmetry codes: (i) $-x,-y,-z+1$; (ii) $x,-y-1 / 2, z-1 / 2$; (iii) $-x+1,-y-1,-z+1$; (iv) $x, y-1, z$; (v) $-x+1,-y,-z+1$; (vi) $x,-y+1 / 2, z+1 / 2$.

Table 12

Coordination modes of the SD anion, showing which atoms form bonds to alkali metal cations.

\begin{tabular}{lllll}
\hline & (I) & (II) & (III) & (III) \\
& Li & Na & K L1 & K L2 \\
Donor atom & & & & \\
O1 & yes & yes & yes & yes \\
O2 & no & no & no & yes \\
N sulfamide & no & yes & yes & yes \\
N ring 1 & yes & yes & no & yes \\
N ring 2 & no & yes & no & yes \\
N amine & no & yes & no & no
\end{tabular}

\section{Acknowledgements}

We thank the National Crystallography Service, University of Southampton, for data collection on (IV) (Coles \& Gale, 2012).

\section{References}

Agilent (2014). CrysAlis PRO. Agilent Technologies Ltd., Yarnton, Oxfordshire, England.

Altomare, A., Cascarano, G., Giacovazzo, C., Guagliardi, A., Burla, M. C., Polidori, G. \& Camalli, M. (1994). J. Appl. Cryst. 27, 435.

Baenziger, N. C. \& Struss, A. W. (1976). Inorg. Chem. 15, 1807-1809.

Buist, A. R., Dennany, L., Kennedy, A. R., Manzie, C., McPhie, K. \& Walker, B. (2014). Acta Cryst. C70, $900-907$.

Coles, S. J. \& Gale, P. A. (2012). Chem. Sci. 3, 683-689.

Elacqua, E., Bucar, D.-K., Henry, R. F., Zhang, G. G. Z. \& MacGillivray, L. R. (2013). Cryst. Growth Des. 13, $393-403$.

Fisher, N. M., Marsh, E. \& Lazova, R. (2003). J. Am. Acad. Dermatol. 49, 730-732.

Ghedini, E., Pizzolitto, C., Albore, G., Menegazzo, F., Signoretto, M., Operti, L. \& Cerrato, G. (2017). J. Sol-Gel Sci. Tech. 83, 618-626. 
Heren, Z., Pasaoglu, H. \& Kastas, G. (2006). Acta Cryst. E62, o3437-o3439.

Kennedy, A. R., Hughes, M. P., Monaghan, M. L., Staunton, E., Teat, S. J. \& Smith, E. W. (2001). J. Chem. Soc. Dalton Trans., 2199-2205.

Kennedy, A. R., Kirkhouse, J. B. \& Whyte, L. (2006). Inorg. Chem. 45, 2965-2971.

Kennedy, A. R., McKellar, S. C. \& Okoth, M. O. (2010). Acta Cryst. E66, m1330-m1331.

Kennedy, A. R., Stewart, H., Eremin, K. \& Stenger, J. (2012). Chem. Eur. J. 18, 3064-3069.

Macrae, C. F., Bruno, I. J., Chisholm, J. A., Edgington, P. R., McCabe, P., Pidcock, E., Rodriguez Monge, L., Taylor, R., van de Streek, J. \& Wood, P. A. (2008). J. Appl. Cryst. 41, 466-470.

Mohseni, M., Shamloo, A., Aghababaei, Z., Vossoughi, M. \& Moravvej, H. (2016). Artificial Organs 40, $765-773$.

Ojala, W. H., Gleason, W. B., Richardson, T. I. \& Lovrien, R. E. (1994a). Acta Cryst. C50, 1615-1620.

Ojala, W. H., Lu, L. K., Albers, K. E., Gleason, W. B., Richardson, T. I., Lovrien, R. E. \& Sudbeck, E. A. (1994b). Acta Cryst. B50, 684-694.

Pan, F., Wang, R. \& Englert, U. (2012). Inorg. Chem. 51, 769-771.

Pan, F., Wang, R. \& Englert, U. (2013). CrystEngComm, 15, 1164-1172.

Preskey, D. \& Kayes, J. B. (1976). J. Clinical Pharm. 1, 39-48.

Sheldrick, G. M. (2015). Acta Cryst. C71, 3-8.

Shi, W.-B., Cui, A.-L. \& Kou, H.-Z. (2015). Polyhedron 99, 252-259.

Sun, J., Xie, C., Zhang, X., Bao, Y., Hou, B., Wang, Z., Gong, J., Hao, H., Wang, Y., Wang, J. \& Yin, Q. (2016). Org. Process Res. Dev. 20, 780-785.

Stahl, P. H. \& Wermuth, C. G. (2008). Eds. Handbook of Pharmaceutical Salts: Properties, Selection and Use. VHCA: Zurich.

Tommasino, J.-B., Renaud, F. N. R., Luneau, D. \& Pilet, G. (2011). Polyhedron 30, 1663-1670.

Yuan, R.-X., Xiong, R.-G., Chen, Z.-F., Zhang, P., Ju, H.-X., Dai, Z., Guo, Z.-J., Fun, H.-K. \& You, X.-Z. (2001). J. Chem. Soc. Dalton Trans., 774-??. 


\title{
checkCIF/PLATON results for paper uk3153
}

\author{
checkCIF/PLATON results
}

Ellipsoid plot

\section{checkCIF/PLATON results}

No syntax errors found. CIF dictionary Interpreting this report

\section{Datablock: I}

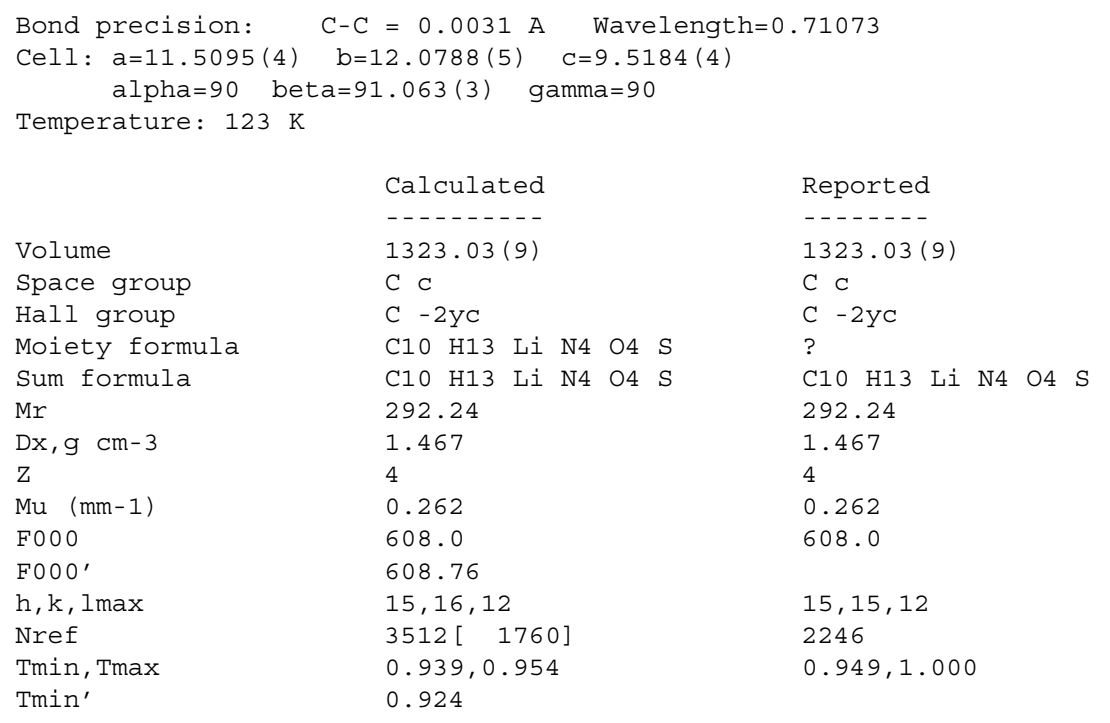

Alert level B

PLAT915_ALERT_3_B No Flack x Check Done: Low Friedel Pair Coverage 34 \%

Alert level $\mathrm{C}$

PLAT048 ALERT 1 C MoietyFormula Not Given (or Incomplete) ........ Please Check PLAT420_ALERT_2_C D-H Without Acceptor N4 N - N $2 \mathrm{~N}$.

Alert level G

PLATO02_ALERT_2_G Number of Distance or Angle Restraints on AtSite PLAT172_ALERT_4_G The CIF-Embedded res File Contains DFIX Records PLAT779_ALERT_4_G Suspect or Irrelevant (Bond) Angle in CIF .... \# $\begin{array}{llllll}\mathrm{O} 1 & -\mathrm{S} 1 & \text {-LI1 } & 1.555 & 1.555 & 1.555\end{array}$ PLAT792_ALERT_1_G Model has Chirality at S1 (Polar SPGR) PLAT860_ALERT_3 G Number of Least-Squares Restraints . . . . . . . . . PLAT910_ALERT_3_G Missing \# of FCF Reflection(s) Below Theta(Min). PLAT912 ALERT 4 G Missing \# of FCF Reflections Above STh/L=0.600 PLAT978_ALERT_2_G Number C-C Bonds with Positive Residual Density.

$\begin{aligned} & 6 \text { Note } \\ & 2 \text { Report } \\ & 18 \text { Check } \\ & 32.38 \text { Deg. } \\ & \text { R Verify } \\ & 8 \text { Note } \\ & 1 \text { Note } \\ & 95 \text { Note } \\ & 10 \text { Info }\end{aligned}$


0 ALERT level $\mathbf{A}=$ Most likely a serious problem - resolve or explain

1 ALERT level B = A potentially serious problem, consider carefully

2 ALERT level $\mathbf{C}=$ Check. Ensure it is not caused by an omission or oversight

8 ALERT level $\mathbf{G}=$ General information/check it is not something unexpected

2 ALERT type 1 CIF construction/syntax error, inconsistent or missing data

3 ALERT type 2 Indicator that the structure model may be wrong or deficient

3 ALERT type 3 Indicator that the structure quality may be low

3 ALERT type 4 Improvement, methodology, query or suggestion

0 ALERT type 5 Informative message, check

\section{Datablock: II}

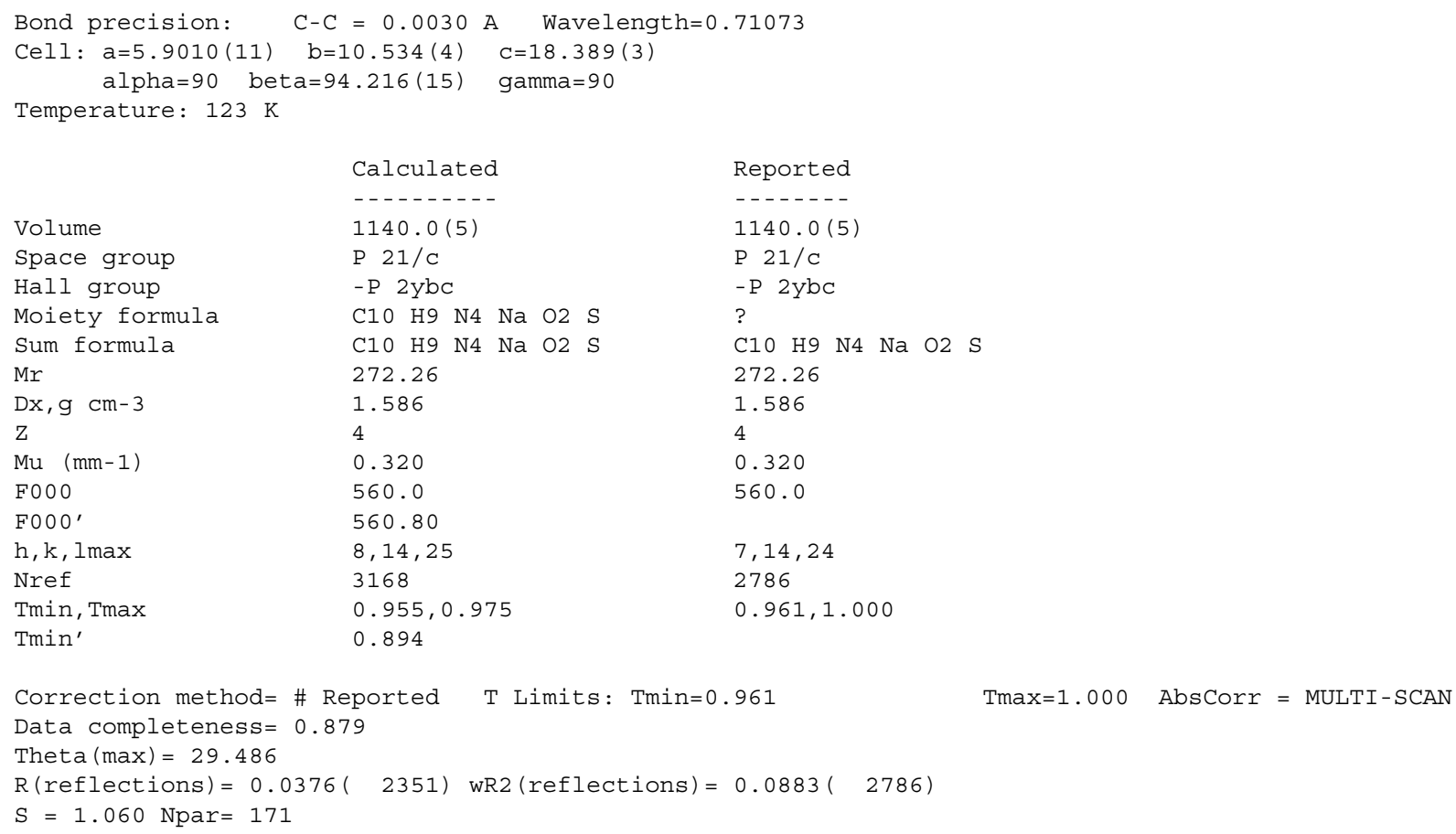

Alert level $\mathrm{C}$

PLAT048_ALERT_1_C MoietyFormula Not Given (or Incomplete) ........ Please Check PLAT790_ALERT_4_C Centre of Gravity not Within Unit Cell: Resd. \# 1 Note C10 H9 N4 Na O2 S

PLAT906_ALERT_3_C Large K Value in the Analysis of Variance ..... 2.164 Check

Alert level G

PLAT004_ALERT_5_G Polymeric Structure Found with Maximum Dimension PLAT764 ALERT 4 G Overcomplete CIF Bond List Detected (Rep/Expd). PLAT793_ALERT_4_G Model has Chirality at S1 (Centro SPGR) PLAT910_ALERT_3_G Missing \# of FCF Reflection(s) Below Theta(Min). PLAT912_ALERT_4_G Missing \# of FCF Reflections Above STh/L=0.600 PLAT978_ALERT_2_G Number C-C Bonds with Positive Residual Density.

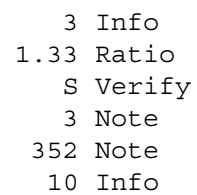

0 ALERT level $\mathbf{A}=$ Most likely a serious problem - resolve or explain

o ALERT level B = A potentially serious problem, consider carefully 
3 ALERT level $\mathbf{C}=$ Check. Ensure it is not caused by an omission or oversight

6 ALERT level $\mathbf{G}=$ General information/check it is not something unexpected

1 ALERT type 1 CIF construction/syntax error, inconsistent or missing data

1 ALERT type 2 Indicator that the structure model may be wrong or deficient

2 ALERT type 3 Indicator that the structure quality may be low

4 ALERT type 4 Improvement, methodology, query or suggestion

1 ALERT type 5 Informative message, check

\section{Datablock: III}

Bond precision: $\quad \mathrm{C}-\mathrm{C}=0.0064 \mathrm{~A} \quad$ Wavelength $=1.54180$

Cell: $a=8.8503(7) \quad b=9.6385(4) \quad c=15.9734(7)$

alpha $=92.350(4) \quad$ beta $=95.186(4) \quad$ gamma $=94.209(4)$

Temperature: $123 \mathrm{~K}$

\begin{tabular}{|c|c|c|}
\hline & Calculated & Reported \\
\hline & -------- & $---\cdots--$ \\
\hline Volume & $1351.79(14)$ & $1351.79(13)$ \\
\hline Space group & $P-1$ & $P-1$ \\
\hline Hall group & $-\mathrm{P} \quad 1$ & $\begin{array}{ll}-\mathrm{P} & 1\end{array}$ \\
\hline Moiety formula & $\mathrm{C} 20$ H26 K2 N8 O8 S2 & $?$ \\
\hline Sum formula & $\mathrm{C} 20 \mathrm{H} 26 \mathrm{~K} 2 \mathrm{~N} 8$ O8 $\mathrm{S} 2$ & $\mathrm{C} 10 \mathrm{H} 13 \mathrm{~K} \quad \mathrm{~N} 4 \mathrm{O} 4 \mathrm{~S}$ \\
\hline $\mathrm{Mr}$ & 648.81 & 324.40 \\
\hline $\mathrm{Dx}, \mathrm{gcm}-3$ & 1.594 & 1.594 \\
\hline $\mathrm{Z}$ & 2 & 4 \\
\hline $\mathrm{Mu}(\mathrm{mm}-1)$ & 5.085 & 5.085 \\
\hline FOO0 & 672.0 & 672.0 \\
\hline F000' & 676.80 & \\
\hline $\mathrm{h}, \mathrm{k}, \mathrm{lmax}$ & $11,12,19$ & $10,11,19$ \\
\hline Nref & 5456 & 5357 \\
\hline Tmin, Tmax & $0.460,0.601$ & $0.601,1.000$ \\
\hline Tmin' & 0.209 & \\
\hline
\end{tabular}

Correction method $=\#$ Reported T Limits: Tmin=0.601

$\operatorname{Tmax}=1.000$ AbsCorr $=$ MULTI - SCAN

Data completeness $=0.982$

Theta $(\max )=73.745$

$\mathrm{R}($ reflections $)=0.0597($ 3951) $\mathrm{wR} 2($ reflections $)=0.1573($ 5357)

$S=1.045$ Npar $=404$

Alert level $B$

PLAT420 ALERT 2 B D-H Without Acceptor O4W --H9W Please Check

Alert level $\mathrm{C}$

PLAT048 ALERT 1 C MoietyFormula Not Given (or Incomplete) ........ PLAT242_ALERT_2_C LOW 'MainMol' Ueq as Compared to Neighbors of

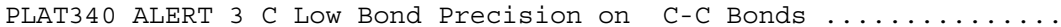
PLAT420_ALERT_2_C D-H Without Acceptor N8 $\quad--\mathrm{H} 4 \mathrm{~N}$ PLAT906 ALERT 3 C Large $K$ Value in the Analysis of Variance ...... PLAT911_ALERT_3_C Missing FCF Refl Between Thmin \& STh/L= 0.600 PLAT934_ALERT_3 C Number of (Iobs-Icalc)/SigmaW > 10 Outliers .... PLAT978_ALERT_2_C Number C-C Bonds with Positive Residual Density.
Please Check

K1 Check

0.00644 Ang.

Please Check

6.059 Check

19 Report

1 Check

0 Info
Alert level G

PLAT002_ALERT_2_G Number of Distance or Angle Restraints on Atsite PLAT004 ALERT 5 G Polymeric Structure Found with Maximum Dimension PLAT045_ALERT_1_G Calculated and Reported Z Differ by a Factor... PLAT154 ALERT 1 G The s.u.'s on the Cell Angles are Equal .. (Note) PLAT172_ALERT_4_G The CIF-Embedded .res File Contains DFIX Records

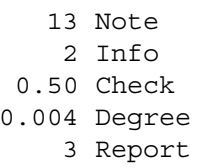

3 Report 
0 ALERT level $\mathbf{A}=$ Most likely a serious problem - resolve or explain

1 ALERT level B = A potentially serious problem, consider carefully

8 ALERT level $\mathbf{C}=$ Check. Ensure it is not caused by an omission or oversight

16 ALERT level $\mathbf{G}=$ General information/check it is not something unexpected

7 ALERT type 1 CIF construction/syntax error, inconsistent or missing data

5 ALERT type 2 Indicator that the structure model may be wrong or deficient

6 ALERT type 3 Indicator that the structure quality may be low

6 ALERT type 4 Improvement, methodology, query or suggestion

1 ALERT type 5 Informative message, check

\section{Datablock: IV}

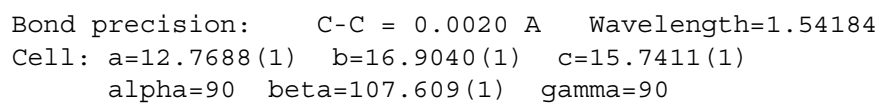

\begin{tabular}{|c|c|c|}
\hline & Calculated & Reported \\
\hline & -------- & ------ \\
\hline Volume & $3238.42(4)$ & $3238.42(4)$ \\
\hline Space group & P $21 / \mathrm{C}$ & P $21 / C$ \\
\hline Hall group & $-\mathrm{P} 2 \mathrm{ybc}$ & $-\mathrm{P} 2 \mathrm{ybc}$ \\
\hline Moiety formula & C32 H36 N4 Na2 O22 S4 ? & \\
\hline Sum formula & C52 H64 N12 Na2 O29 S6 & C26 H32 N6 Na $014.50 \mathrm{~S} 3$ \\
\hline Mr & 1559.49 & 779.74 \\
\hline $\mathrm{Dx}, \mathrm{g} \mathrm{cm}-3$ & 1.599 & 1.599 \\
\hline $\mathrm{Z}$ & 2 & 4 \\
\hline $\mathrm{Mu} \quad(\mathrm{mm}-1)$ & 2.946 & 2.946 \\
\hline FO00 & 1620.0 & 1620.0 \\
\hline Fo00' & 1629.77 & \\
\hline $\mathrm{h}, \mathrm{k}, \mathrm{lmax}$ & $15,20,18$ & $15,20,18$ \\
\hline Nref & 5935 & 5913 \\
\hline Tmin, Tmax & $0.754,0.889$ & $0.615,1.000$ \\
\hline Tmin' & 0.702 & \\
\hline
\end{tabular}

Correction method= \# Reported T Limits: Tmin=0.615 Tmax=1.000 AbsCorr = MULTI-SCAN Data completeness $=0.996$

Theta $(\max )=68.245$

$\mathrm{R}$ (reflections) $=0.0280($ 5686) $\mathrm{wR} 2($ reflections $)=0.0745($ 5913)

$\mathrm{S}=1.048$ Npar $=537$

Alert level $B$

PLAT416_ALERT_2_B Short Intra D-H.H-D H11W ..H13W

Alert level C

PLAT048_ALERT_1_C MoietyFormula Not Given (or Incomplete) ....... Please Check 
Alert level G

PLATO02_ALERT_2_G Number of Distance or Angle Restraints on AtSite PLAT045_ALERT_1_G Calculated and Reported Z Differ by a Factor...

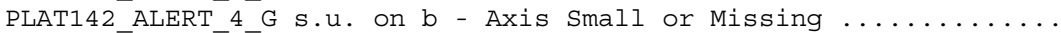
PLAT143_ALERT_4_G s.u. on c - Axis Small or Missing ........... PLAT172 ALERT 4 G The CIF-Embedded .res File Contains DFIX Records PLAT300_ALERT_4_G Atom Site Occupancy of H8W Constrained at PLAT300_ALERT_4_G Atom Site Occupancy of H14W Constrained at PLAT300_ALERT_4_G Atom Site Occupancy of O6W Constrained at PLAT300_ALERT 4 G Atom Site Occupancy of 07W Constrained at PLAT300_ALERT_4_G Atom Site Occupancy of H12W Constrained at PLAT300 ALERT 4_G Atom Site Occupancy of H13W Constrained at PLAT300_ALERT_4_G Atom Site Occupancy of O5W Constrained at PLAT300 ALERT 4 G Atom Site Occupancy of H9W Constrained at PLAT300_ALERT_4_G Atom Site Occupancy of H1OW Constrained at PLAT302 ALERT 4 G Anion/Solvent/Minor-Residue Disorder (Resd 3 ) PLAT302_ALERT_4_G Anion/Solvent/Minor-Residue Disorder (Resd 4 ) PLAT304_ALERT_4_G Non-Integer Number of Atoms in ...... Resd 4 PLAT790_ALERT_4_G Centre of Gravity not Within Unit Cell: Resd. \# $\mathrm{H} 2 \mathrm{O}$

PLAT790_ALERT_4_G Centre of Gravity not Within Unit Cell: Resd. \# $\mathrm{H} 2 \mathrm{O}$

PLAT860_ALERT_3_G Number of Least-Squares Restraints ........... PLAT912 ALERT 4 G Missing \# of FCF Reflections Above STh/L=0.600 PLAT933_ALERT_2_G Number of OMIT Records in Embedded .res File... PLAT978_ALERT_2_G Number C-C Bonds with Positive Residual Density.

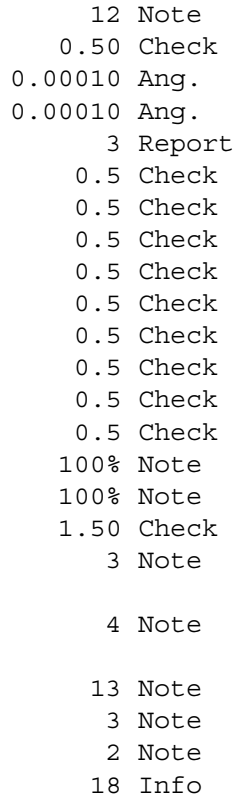

\footnotetext{
0 ALERT level $\mathbf{A}=$ Most likely a serious problem - resolve or explain

1 ALERT level $B=A$ potentially serious problem, consider carefully

3 ALERT level $\mathbf{C}=$ Check. Ensure it is not caused by an omission or oversight
}

23 ALERT level $\mathbf{G}=$ General information/check it is not something unexpected

2 ALERT type 1 CIF construction/syntax error, inconsistent or missing data

4 ALERT type 2 Indicator that the structure model may be wrong or deficient

3 ALERT type 3 Indicator that the structure quality may be low

18 ALERT type 4 Improvement, methodology, query or suggestion

0 ALERT type 5 Informative message, check

\section{Datablock: V}

Bond precision: $\quad \mathrm{C}-\mathrm{C}=0.0028 \mathrm{~A} \quad$ Wavelength $=0.71073$

Cell: $a=8.5796(2) \quad b=19.0371(5) \quad c=11.2512(4)$ alpha $=90$ beta $=121.116(3) \quad$ gamma $=90$ Temperature: $123 \mathrm{~K}$

\begin{tabular}{|c|c|c|}
\hline & Calculated & Reported \\
\hline & -------- & ------- \\
\hline Volume & $1573.27(9)$ & $1573.27(9)$ \\
\hline Space group & P $21 / C$ & P $21 / C$ \\
\hline Hall group & $-\mathrm{P} 2 \mathrm{ybc}$ & $-\mathrm{P} 2 \mathrm{ybc}$ \\
\hline Moiety formula & C10 H9 N4 O2 S, C5 H7 N2 & $?$ \\
\hline Sum formula & $\mathrm{C} 15 \mathrm{H} 16 \mathrm{~N} 6 \mathrm{O} 2 \mathrm{~S}$ & $\mathrm{C} 15 \mathrm{H} 16 \mathrm{~N} 6 \mathrm{O} 2 \mathrm{~S}$ \\
\hline Mr & 344.40 & 344.40 \\
\hline $\mathrm{Dx}, \mathrm{g} \mathrm{cm}-3$ & 1.454 & 1.454 \\
\hline $\mathrm{Z}$ & 4 & 4 \\
\hline $\mathrm{Mu} \quad(m m-1)$ & 0.228 & 0.228 \\
\hline F000 & 720.0 & 720.0 \\
\hline $\mathrm{F} 000^{\prime}$ & 720.74 & \\
\hline$h, k, l \max$ & $11,25,15$ & $11,25,15$ \\
\hline Nref & 4183 & 4082 \\
\hline Tmin, Tmax & $0.921,0.995$ & $0.527,1.000$ \\
\hline
\end{tabular}


Theta $(\max )=28.994$

$\mathrm{R}$ (reflections) $=0.0445(3225) \quad \mathrm{wR} 2($ reflections $)=0.1139(4082)$

$S=1.040$ Npar $=237$

Alert level $\mathrm{C}$

PLAT048_ALERT_1_C MoietyFormula Not Given (or Incomplete) ....... Please Check PLAT906 ALERT 3 C Large $\mathrm{K}$ Value in the Analysis of Variance ...... 2.533 Check

Alert level G

PLAT128_ALERT_4_G Alternate setting for Input Space Group P21/C PLAT790_ALERT_4_G Centre of Gravity not Within Unit Cell: Resd. \# C5 H7 N2

PLAT910_ALERT_3_G Missing \# of FCF Reflection(s) Below Theta(Min). PLAT912_ALERT_4_G Missing \# of FCF Reflections Above STh/L=0.600 PLAT978_ALERT_2_G Number C-C Bonds with Positive Residual Density.

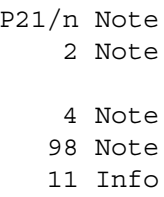

o ALERT level $\mathbf{A}=$ Most likely a serious problem - resolve or explain

o ALERT level B = A potentially serious problem, consider carefully

2 ALERT level $\mathbf{C}=$ Check. Ensure it is not caused by an omission or oversight

5 ALERT level $\mathbf{G}=$ General information/check it is not something unexpected

1 ALERT type 1 CIF construction/syntax error, inconsistent or missing data

1 ALERT type 2 Indicator that the structure model may be wrong or deficient

2 ALERT type 3 Indicator that the structure quality may be low

3 ALERT type 4 Improvement, methodology, query or suggestion

0 ALERT type 5 Informative message, check

\section{Datablock: VI}

Bond precision: $\quad \mathrm{C}-\mathrm{C}=0.0020 \mathrm{~A} \quad$ Wavelength $=0.71073$
Cell: $\mathrm{a}=12.7755(4) \quad \mathrm{b}=9.8979(4) \quad \mathrm{C}=11.5366(4)$

alpha $=90$ beta $=93.508(3) \quad$ gamma $=90$

Temperature: $123 \mathrm{~K}$

\begin{tabular}{|c|c|c|}
\hline & Calculated & Reported \\
\hline & -------- & ------- \\
\hline Volume & $1456.08(9)$ & $1456.08(9)$ \\
\hline Space group & P $21 / C$ & P $21 / C$ \\
\hline Hall group & $-\mathrm{P} 2 \mathrm{ybc}$ & $-\mathrm{P} 2 \mathrm{ybc}$ \\
\hline Moiety formula & $\mathrm{C} 10 \mathrm{H} 9 \mathrm{~N} 4 \mathrm{O} 2 \mathrm{~S}, \mathrm{C} 2 \mathrm{H} 8$ ? & \\
\hline Sum formula & C12 H19 N5 O4 S & C12 H19 N5 O4 S \\
\hline $\mathrm{Mr}$ & 329.38 & 329.38 \\
\hline $\mathrm{Dx}, \mathrm{gcm}-3$ & 1.503 & 1.503 \\
\hline $\mathrm{Z}$ & 4 & 4 \\
\hline $\mathrm{Mu} \quad(\mathrm{mm}-1)$ & 0.250 & 0.250 \\
\hline F000 & 696.0 & 696.0 \\
\hline F000' & 696.79 & \\
\hline $\mathrm{h}, \mathrm{k}, \mathrm{lmax}$ & $17,13,16$ & $17,12,15$ \\
\hline Nref & 4052 & 3571 \\
\hline Tmin, Tmax & $0.939,0.970$ & $0.904,1.000$ \\
\hline Tmin' & 0.939 & \\
\hline
\end{tabular}

Correction method= \# Reported T Limits: Tmin=0.904

$\operatorname{Tmax}=1.000$ AbsCorr $=$ MULTI - SCAN

Data completeness $=0.881$

Theta $(\max )=29.531$ 
Alert level $\mathrm{C}$

PLAT048_ALERT_1_C MoietyFormula Not Given (or Incomplete) ....... Please Check

Alert level G

PLAT790 ALERT 4 G Centre of Gravity not Within Unit Cell: Resd. \# 2 Note $\mathrm{C} 2 \mathrm{H} 8 \mathrm{~N} O$

PLAT910 ALERT 3 G Missing \# of FCF Reflection(s) Below Theta(Min). 4 Note

PLAT912_ALERT_4_G Missing \# of FCF Reflections Above STh/L= $0.600 \quad 432$ Note

PLAT978 ALERT 2 G Number C-C Bonds with Positive Residual Density. 9 Info

0 ALERT level $\mathbf{A}=$ Most likely a serious problem - resolve or explain

0 ALERT level B = A potentially serious problem, consider carefully

1 ALERT level $\mathbf{C}=$ Check. Ensure it is not caused by an omission or oversight

4 ALERT level $\mathbf{G}=$ General information/check it is not something unexpected

1 ALERT type 1 CIF construction/syntax error, inconsistent or missing data

1 ALERT type 2 Indicator that the structure model may be wrong or deficient

1 ALERT type 3 Indicator that the structure quality may be low

2 ALERT type 4 Improvement, methodology, query or suggestion

0 ALERT type 5 Informative message, check

\section{database duplication summary}

\section{Datablock: I}

- Chemical name $=$

- $R$ factor $=0.026$

- Space group =

- Formula $=$ C10 H13 Li N4 O4 S

- $\mathrm{a}=11.5095 \mathrm{~b}=12.0788 \quad \mathrm{c}=9.5184$

- alpha $=90$ beta $=91.063$ gamma $=90$

\section{Datablock: II}

- Chemical name =

- $R$ factor $=0.038$

- Space group =

- Formula $=\mathrm{C} 10 \mathrm{H} 9 \mathrm{~N} 4 \mathrm{Na} \mathrm{O} 2 \mathrm{~S}$

- $\mathrm{a}=5.901 \quad \mathrm{~b}=10.534 \quad \mathrm{c}=18.389$

- alpha $=90$ beta $=94.216$ gamma $=90$

\section{Datablock: III}

- Chemical name =

- $R$ factor $=0.060$ 
- Space group $=$

- Formula $=\mathrm{C} 10 \mathrm{H} 13 \mathrm{~K}$ N4 O4 S

- $a=8.8503 \quad b=9.6385 \quad c=15.9734$

- alpha=92.35 beta=95.186 gamma=94.209

\section{Datablock: IV}

- Chemical name =

- $R$ factor $=0.028$

- Space group =

- Formula $=$ C26 H32 N6 Na O14.50 S3

- $a=12.7688 \quad b=16.904 \quad c=15.7411$

- alpha $=90$ beta $=107.609$ gamma $=90$

\section{Datablock: V}

- Chemical name $=$

- $R$ factor $=0.045$

- Space group =

- Formula $=$ C15 H16 N6 O2 S

- $a=8.5796 \quad b=19.0371 \quad c=11.2512$

- alpha $=90$ beta $=121.116$ gamma $=90$

\section{Datablock: VI}

- Chemical name $=$

- $R$ factor $=0.037$

- Space group =

- Formula $=\mathrm{C} 12 \mathrm{H} 19 \mathrm{~N} 5 \mathrm{O} 4 \mathrm{~S}$

- $a=12.7755 \quad b=9.8979 \quad c=11.5366$

- alpha $=90$ beta $=93.508$ gamma $=90$

\section{No duplication found.}

\section{reference checking results}

The following references were not checked in detail as they were not recognized as journal references

Agilent (2014). CrysalisPro. Agilent Technologies Ltd., Yarnton, Oxfordshire, England.

Stahl, P. H. \& Wermuth, C. G. (2008). Eds. Handbook of Pharmaceutical Salts: Properties, Selection and Use. VHCA: Zurich.

The following references may be incorrectly formatted

Ghedini, E., Pizzolitto, C., Albore, G., Menegazzo, F., Signoretto, M., Operti, L. \& Cerrato, G. (2017). J. Sol-Gel Sci. Tech. 83, 618--626.

[Unrecognized journal title.] 
Preskey, D. \& Kayes, J. B. (1976). J. Clinical Pharm. 1, 39--48.

[Unrecognized journal title.]

\section{Results of online verification of references to IUCr journals}

More than one match found for Altomare, A., Cascarano, G., Giacovazzo, C., Guagliardi, A., Burla, M. C., Polidori, G. \& Camalli, M. (1994). J. Appl. Cryst. 27, 435--436. \#ATL:SIRPOW.92 - a program for automatic solution of crystal structures by direct methods optimized for powder data. [CNOR:wi0150]\# - please check.

\section{All references appear to be cited unambiguously}

\section{Citation comments}

1 date found in data_I_exptl_absorpt_process_details that could be part of a citation but not found in reference list: 2010

1 date found in data_II_exptl_absorpt_process_details that could be part of a citation but not found in reference list: 2010

1 date found in data_III_exptl_absorpt_process_details that could be part of a citation but not found in reference list: 2014

1 date found in data_IV_exptl_absorpt_process_details that could be part of a citation but not found in reference list: 2017

1 date found in data_V_exptl_absorpt_process_details that could be part of a citation but not found in reference list: 2010

1 date found in data_VI_exptl_absorpt_process_details that could be part of a citation but not found in reference list: 2010 

Datablock I - ellipsoid plot

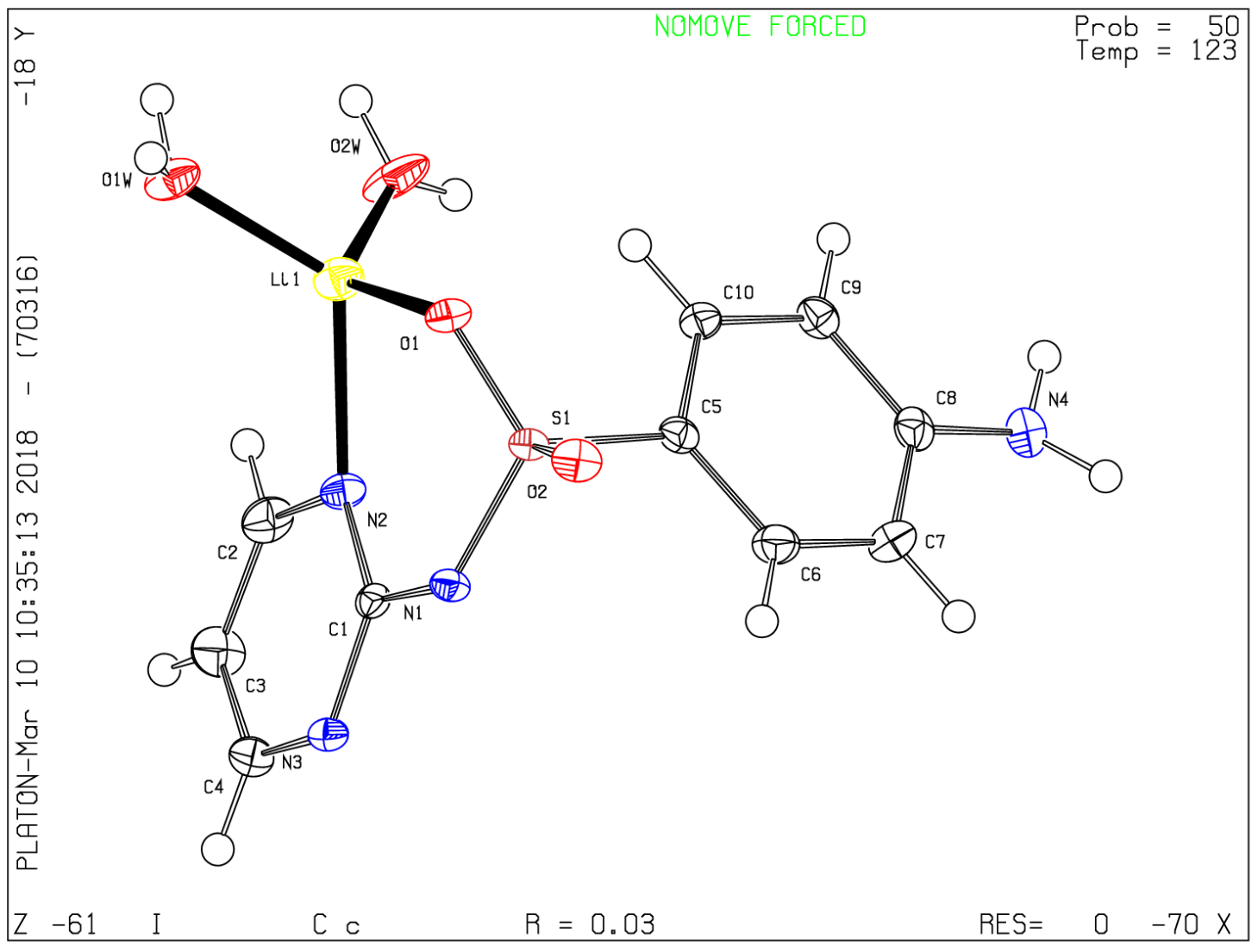


Datablock II - ellipsoid plot

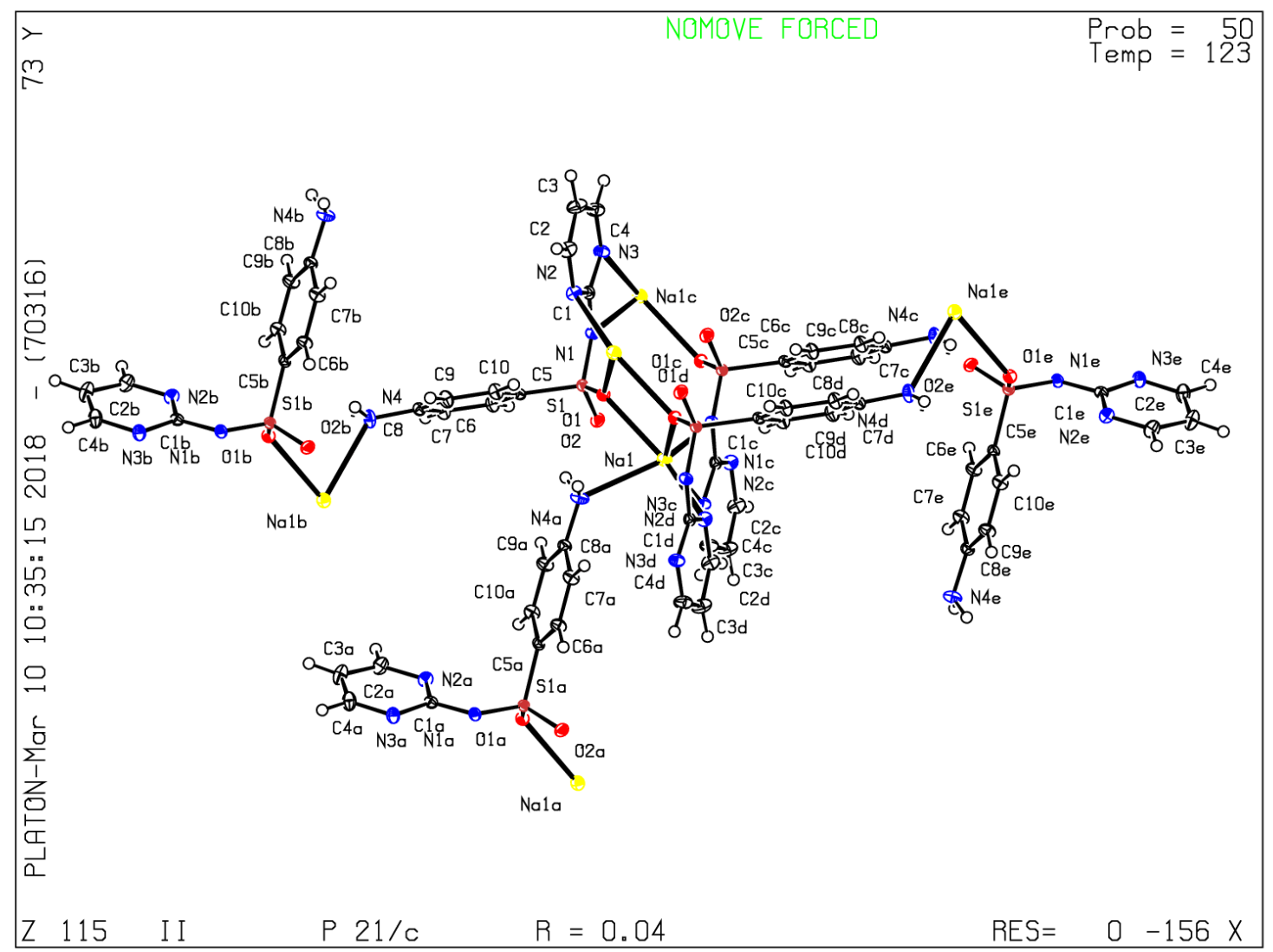


Datablock III - ellipsoid plot

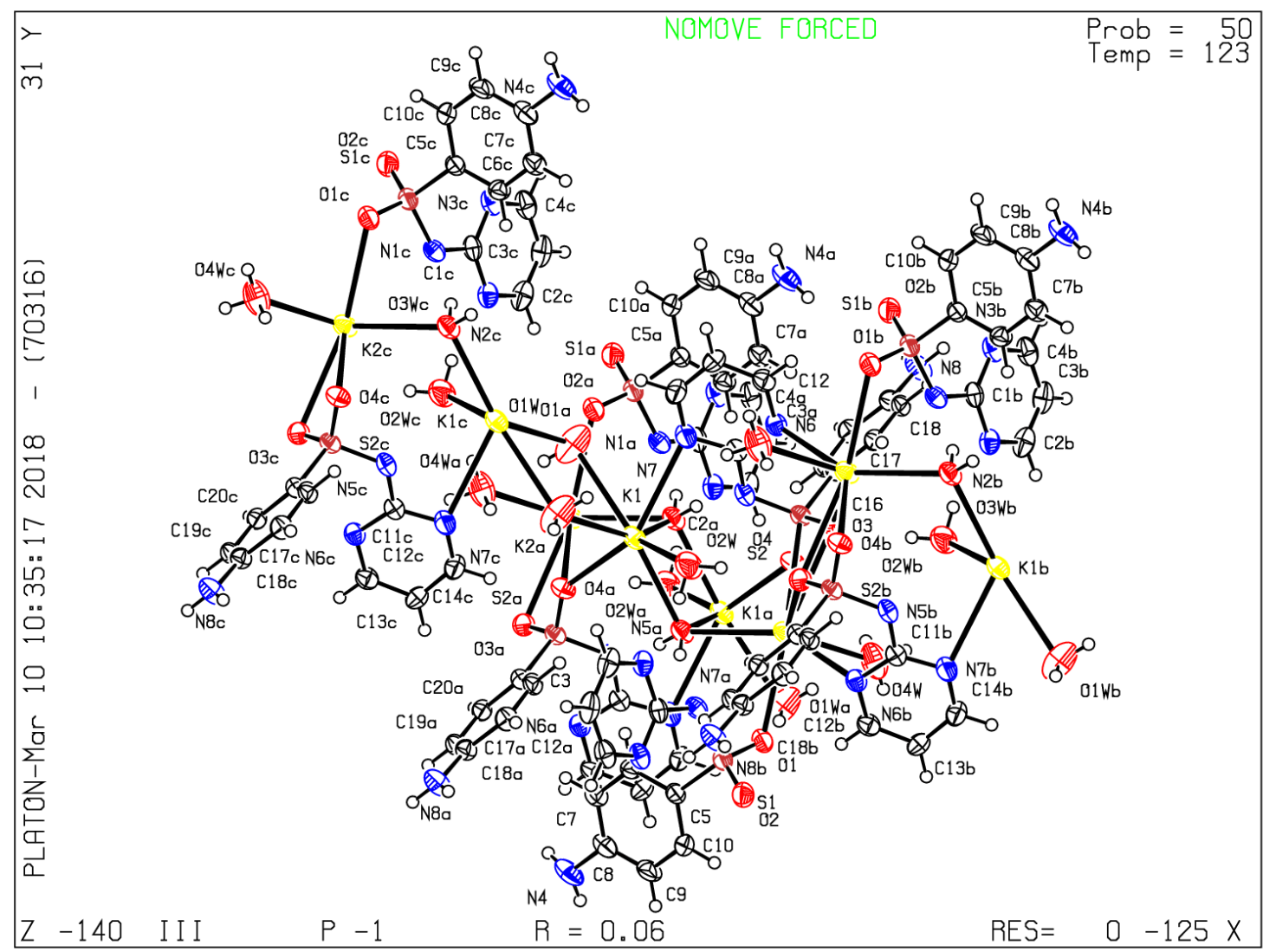


Datablock IV - ellipsoid plot

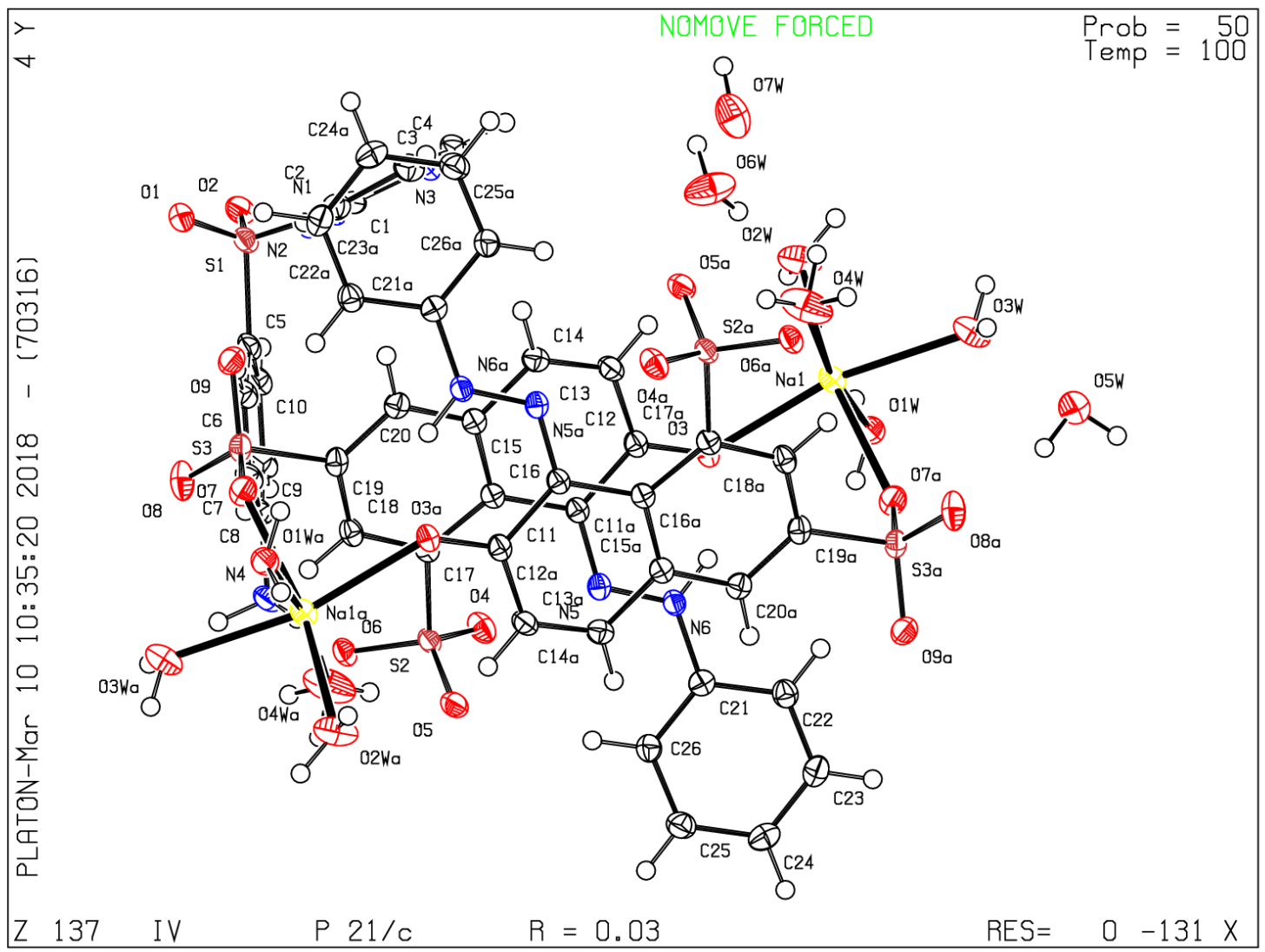


Datablock V - ellipsoid plot

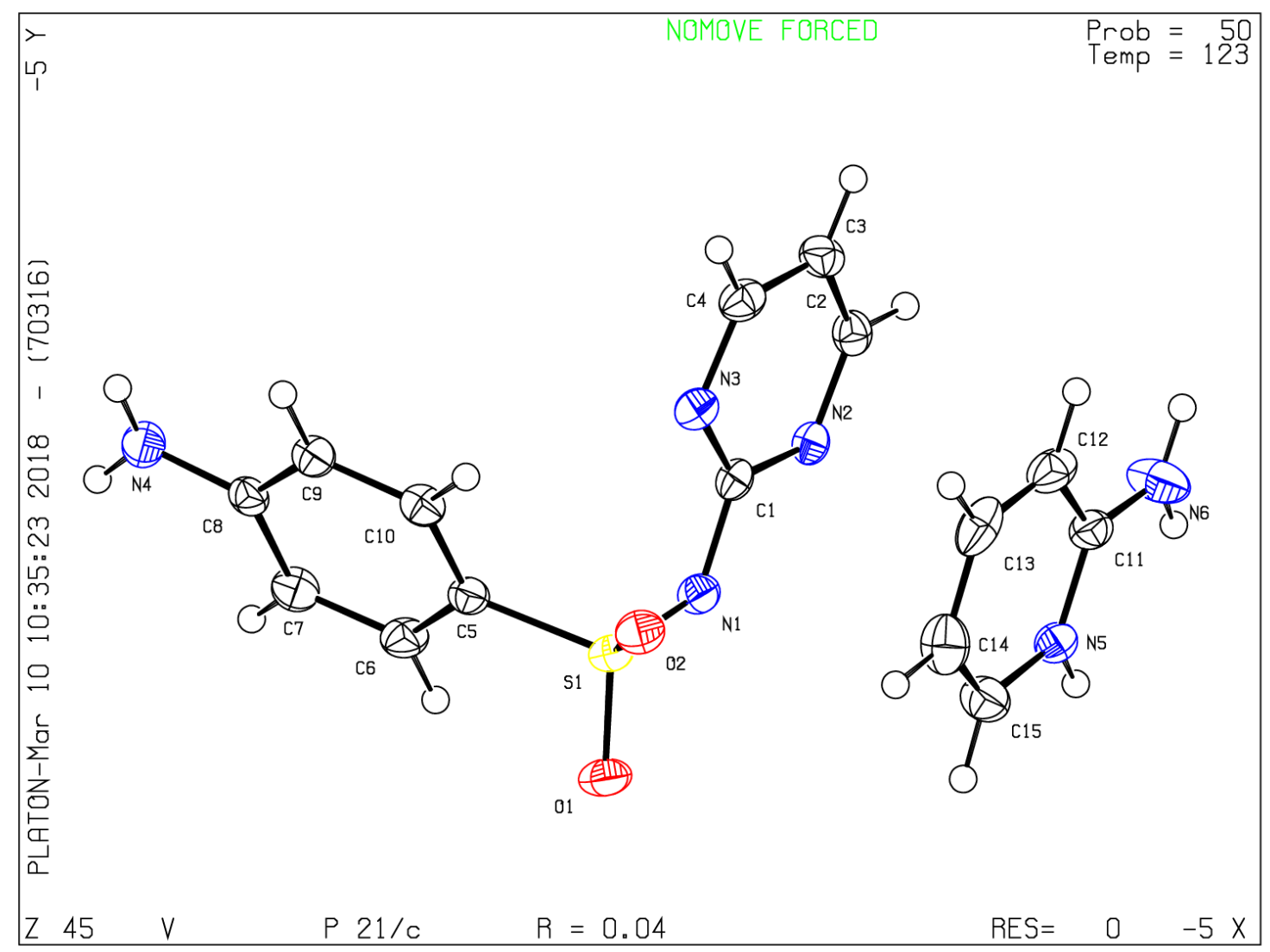


Datablock VI - ellipsoid plot

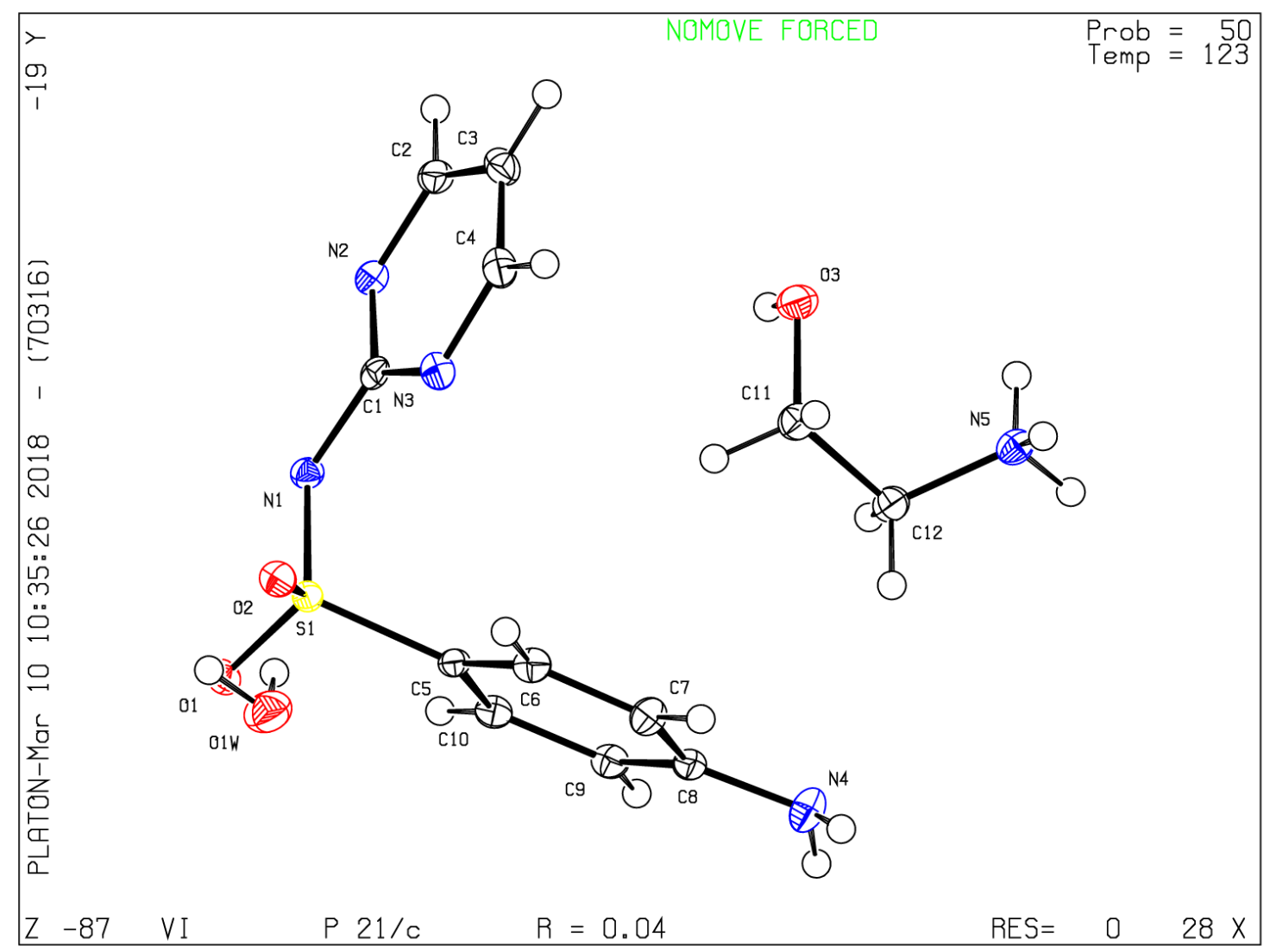




\section{supporting information}

2 Salt forms of sulfadiazine with alkali metal and organic cations.

3 Gemma Campbell, Rebecca Fisher, Alan R. Kennedy,* Nathan L. C. King and Rebecca Spiteri

\section{Computing details}

For all structures, data collection: CrysAlis PRO (Agilent, 2014); cell refinement: CrysAlis PRO; data reduction: CrysAlis PRO. Program(s) used to solve structure: SIR92 (Altomare et al., 1994) for (I), (III), (IV); SHELXS (Sheldrick, 2015) for (II), (V), (VI). For all structures, program(s) used to refine structure: SHELXL2014 (Sheldrick, 2015); molecular graphics: Mercury (Macrae et al., 2008); software used to prepare material for publication: SHELXL2014.

(I)

$$
\begin{aligned}
& \text { Crystal data } \\
& \mathrm{C}_{10} \mathrm{H}_{13} \mathrm{LiN}_{4} \mathrm{O}_{4} \mathrm{~S} \\
& M_{r}=292.24 \\
& \text { Monoclinic, Cc } \\
& a=11.5095(4) \AA \\
& b=12.0788(5) \AA \\
& c=9.5184(4) \AA \\
& \beta=91.063(3)^{\circ} \\
& V=1323.03(9) \AA^{3} \\
& Z=4
\end{aligned}
$$

\section{Data collection}

Oxford Diffraction Xcalibur E diffractometer

Radiation source: tube $\omega$ scans

$$
\begin{aligned}
& F(000)=608 \\
& D_{\mathrm{x}}=1.467 \mathrm{Mg} \mathrm{m}^{-3} \\
& \text { Mo } K \alpha \text { radiation, } \lambda=0.71073 \AA \\
& \text { Cell parameters from } 2629 \text { reflections } \\
& \theta=3.2-29.8^{\circ} \\
& \mu=0.26 \mathrm{~mm}^{-1} \\
& T=123 \mathrm{~K} \\
& \text { Block, colourless } \\
& 0.30 \times 0.20 \times 0.18 \mathrm{~mm}
\end{aligned}
$$

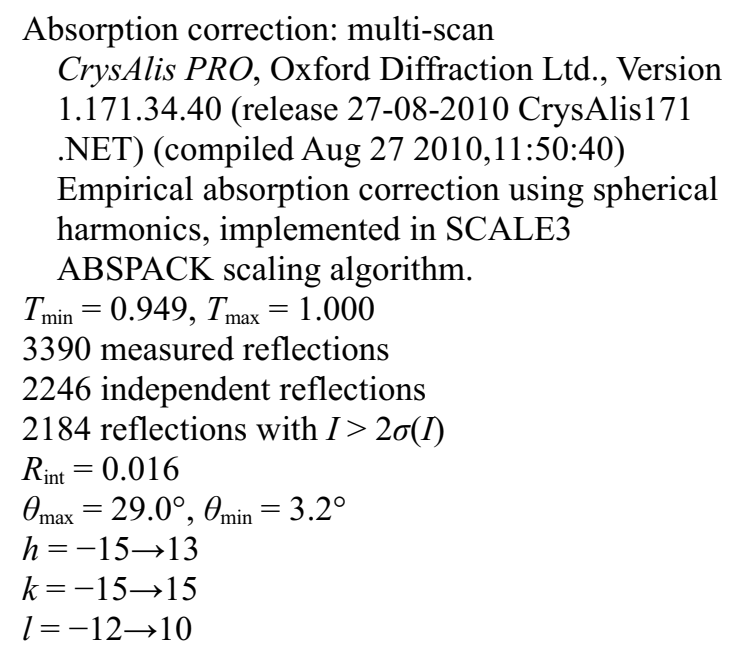

2246 reflections

207 parameters

8 restraints

Hydrogen site location: mixed

\section{Refinement}

Refinement on $F^{2}$

Least-squares matrix: full

$R\left[F^{2}>2 \sigma\left(F^{2}\right)\right]=0.026$

$w R\left(F^{2}\right)=0.066$

$S=1.04$ 
$\mathrm{H}$ atoms treated by a mixture of independent and constrained refinement

$w=1 /\left[\sigma^{2}\left(F_{\mathrm{o}}^{2}\right)+(0.0365 P)^{2}+0.3087 P\right]$

where $P=\left(F_{\mathrm{o}}^{2}+2 F_{\mathrm{c}}^{2}\right) / 3$

$(\Delta / \sigma)_{\max }<0.001$

$\Delta \rho_{\max }=0.28$ e $\AA^{-3}$

$\Delta \rho_{\min }=-0.25$ e $\AA^{-3}$
Extinction correction: SHELXL2014 (Sheldrick, 2015), $\mathrm{Fc}^{*}=\mathrm{kFc}\left[1+0.001 \times \mathrm{xF}^{2} \lambda^{3} / \sin (2 \theta)\right]^{-1 / 4}$

Extinction coefficient: 0.0054 (9)

Absolute structure: Refined as an inversion twin.

Absolute structure parameter: -0.01 (8)

Special details

Geometry. All e.s.d.'s (except the e.s.d. in the dihedral angle between two 1.s. planes) are estimated using the full covariance matrix. The cell e.s.d.'s are taken into account individually in the estimation of e.s.d.'s in distances, angles and torsion angles; correlations between e.s.d.'s in cell parameters are only used when they are defined by crystal symmetry. An approximate (isotropic) treatment of cell e.s.d.'s is used for estimating e.s.d.'s involving 1.s. planes.

Refinement. Refined as a 2-component inversion twin.

Fractional atomic coordinates and isotropic or equivalent isotropic displacement parameters $\left(\hat{A}^{2}\right)$

\begin{tabular}{|c|c|c|c|c|}
\hline & $x$ & $y$ & $z$ & $U_{\text {iso }} * / U_{\text {eq }}$ \\
\hline Li1 & $0.8416(4)$ & $0.2755(4)$ & $0.4134(5)$ & $0.0198(8)$ \\
\hline $\mathrm{S} 1$ & $0.75432(4)$ & $0.33089(4)$ & $0.70030(5)$ & $0.01208(13)$ \\
\hline O1 & $0.81653(14)$ & $0.36761(14)$ & $0.57624(17)$ & $0.0157(3)$ \\
\hline $\mathrm{O} 2$ & $0.72513(14)$ & $0.42112(14)$ & $0.79429(18)$ & $0.0175(4)$ \\
\hline $\mathrm{O} 1 \mathrm{~W}$ & $0.83115(15)$ & $0.36603(15)$ & $0.24923(19)$ & $0.0211(4)$ \\
\hline $\mathrm{O} 2 \mathrm{~W}$ & $0.97330(16)$ & 0.19039 (18) & $0.3764(2)$ & $0.0314(5)$ \\
\hline N1 & $0.63739(15)$ & $0.26680(16)$ & $0.6691(2)$ & $0.0129(4)$ \\
\hline N2 & $0.71240(17)$ & $0.16691(16)$ & $0.4758(2)$ & $0.0154(4)$ \\
\hline N3 & $0.52506(17)$ & $0.13140(17)$ & $0.5695(2)$ & $0.0145(4)$ \\
\hline N4 & $1.05266(19)$ & $0.00920(18)$ & $1.0096(2)$ & $0.0187(4)$ \\
\hline $\mathrm{C} 1$ & 0.62734 (19) & 0.18727 (19) & $0.5688(2)$ & $0.0121(4)$ \\
\hline $\mathrm{C} 2$ & $0.6930(2)$ & $0.0854(2)$ & 0.3827 & $0.0206(5)$ \\
\hline $\mathrm{H} 2$ & 0.7523 & 0.0684 & 0.3180 & $0.025^{*}$ \\
\hline $\mathrm{C} 3$ & $0.5921(2)$ & $0.0254(2)$ & $0.3763(3)$ & $0.0231(5)$ \\
\hline $\mathrm{H} 3$ & 0.5798 & -0.0317 & 0.3091 & $0.028 *$ \\
\hline $\mathrm{C} 4$ & $0.5093(2)$ & $0.0531(2)$ & 0.4735 & $0.0184(5)$ \\
\hline $\mathrm{H} 4$ & 0.4376 & 0.0141 & 0.4714 & $0.022 *$ \\
\hline $\mathrm{C} 5$ & 0.84694 (19) & $0.23972(19)$ & $0.7933(2)$ & $0.0137(4)$ \\
\hline C6 & $0.8089(2)$ & $0.1949(2)$ & $0.9188(3)$ & $0.0189(5)$ \\
\hline H6 & 0.7352 & 0.2151 & 0.9541 & $0.023 *$ \\
\hline $\mathrm{C} 7$ & $0.8785(2)$ & $0.1211(2)$ & $0.9918(2)$ & $0.0198(5)$ \\
\hline $\mathrm{H} 7$ & 0.8523 & 0.0904 & 1.0775 & $0.024 *$ \\
\hline $\mathrm{C} 8$ & $0.9871(2)$ & 0.09088 (19) & $0.9410(2)$ & $0.0161(5)$ \\
\hline $\mathrm{C} 9$ & $1.0257(2)$ & $0.1391(2)$ & $0.8169(2)$ & $0.0157(5)$ \\
\hline H9 & 1.1005 & 0.1211 & 0.7830 & $0.019 *$ \\
\hline $\mathrm{C} 10$ & $0.95556(18)$ & 0.21289 (19) & $0.7430(2)$ & $0.0154(5)$ \\
\hline $\mathrm{H} 10$ & 0.9819 & 0.2450 & 0.6582 & $0.018^{*}$ \\
\hline H1W & $0.815(3)$ & $0.4368(12)$ & $0.251(4)$ & $0.050(11)^{*}$ \\
\hline $\mathrm{H} 2 \mathrm{~W}$ & $0.890(2)$ & $0.365(3)$ & $0.194(3)$ & $0.055(12)^{*}$ \\
\hline H3W & $1.017(3)$ & $0.202(3)$ & $0.305(3)$ & $0.063(12)^{*}$ \\
\hline $\mathrm{H} 4 \mathrm{~W}$ & $1.002(3)$ & $0.131(2)$ & $0.413(4)$ & $0.061(12)^{*}$ \\
\hline
\end{tabular}




\begin{tabular}{lllll}
$\mathrm{H} 1 \mathrm{~N}$ & $1.122(3)$ & $0.003(3)$ & $0.985(3)$ & $0.025(8)^{*}$ \\
$\mathrm{H} 2 \mathrm{~N}$ & $1.044(2)$ & $0.012(2)$ & $1.107(3)$ & $0.017(7)^{*}$ \\
\hline
\end{tabular}

Atomic displacement parameters $\left(\AA^{2}\right)$

\begin{tabular}{lllllll}
\hline & $U^{11}$ & $U^{22}$ & $U^{33}$ & $U^{12}$ & $U^{13}$ & $U^{33}$ \\
\hline Li1 & $0.019(2)$ & $0.019(2)$ & $0.022(2)$ & $0.0013(16)$ & $0.0043(15)$ & $0.0013(17)$ \\
S1 & $0.0108(2)$ & $0.0113(2)$ & $0.0143(2)$ & $-0.0013(2)$ & $0.00227(16)$ & $-0.0006(2)$ \\
O1 & $0.0157(8)$ & $0.0145(8)$ & $0.0171(8)$ & $-0.0029(6)$ & $0.0045(6)$ & $0.0013(7)$ \\
O2 & $0.0178(8)$ & $0.0133(8)$ & $0.0213(9)$ & $0.0001(6)$ & $0.0032(6)$ & $-0.0042(7)$ \\
O1W & $0.0208(9)$ & $0.0181(9)$ & $0.0246(9)$ & $0.0048(7)$ & $0.0098(7)$ & $0.0059(8)$ \\
O2W & $0.0274(11)$ & $0.0332(11)$ & $0.0345(12)$ & $0.0151(8)$ & $0.0209(9)$ & $0.0194(9)$ \\
N1 & $0.0100(9)$ & $0.0144(9)$ & $0.0145(9)$ & $-0.0011(7)$ & $0.0020(6)$ & $-0.0011(8)$ \\
N2 & $0.0140(10)$ & $0.0165(10)$ & $0.0160(10)$ & $0.0001(8)$ & $0.0049(7)$ & $-0.0007(8)$ \\
N3 & $0.0135(9)$ & $0.0149(10)$ & $0.0149(9)$ & $-0.0035(8)$ & $0.0013(7)$ & $0.0015(8)$ \\
N4 & $0.0194(11)$ & $0.0187(11)$ & $0.0180(11)$ & $0.0018(8)$ & $-0.0032(8)$ & $-0.0010(8)$ \\
C1 & $0.0126(10)$ & $0.0117(10)$ & $0.0120(11)$ & $0.0003(8)$ & $0.0004(7)$ & $0.0030(8)$ \\
C2 & $0.0230(12)$ & $0.0219(12)$ & $0.0172(12)$ & $0.0010(10)$ & $0.0071(9)$ & $-0.0048(10)$ \\
C3 & $0.0262(13)$ & $0.0218(12)$ & $0.0214(13)$ & $-0.0044(10)$ & $0.0025(9)$ & $-0.0108(11)$ \\
C4 & $0.0171(11)$ & $0.0182(12)$ & $0.0198(12)$ & $-0.0042(9)$ & $-0.0005(8)$ & $0.0008(10)$ \\
C5 & $0.0127(10)$ & $0.0133(11)$ & $0.0150(11)$ & $-0.0018(8)$ & $0.0001(8)$ & $-0.0019(9)$ \\
C6 & $0.0157(11)$ & $0.0212(12)$ & $0.0200(13)$ & $-0.0016(10)$ & $0.0029(9)$ & $0.0004(10)$ \\
C7 & $0.0215(12)$ & $0.0208(12)$ & $0.0174(12)$ & $-0.0014(10)$ & $0.0049(9)$ & $0.0030(10)$ \\
C8 & $0.0184(11)$ & $0.0142(11)$ & $0.0157(12)$ & $-0.0026(9)$ & $-0.0030(8)$ & $-0.0031(9)$ \\
C9 & $0.0129(11)$ & $0.0176(12)$ & $0.0167(11)$ & $0.0018(9)$ & $0.0004(8)$ & $-0.0036(9)$ \\
C10 & $0.0139(11)$ & $0.0177(12)$ & $0.0145(11)$ & $-0.0003(9)$ & $0.0027(8)$ & $0.0000(9)$
\end{tabular}

Geometric parameters $(\AA, \stackrel{o}{)}$

\begin{tabular}{|c|c|c|c|}
\hline $\mathrm{Li} 1-\mathrm{O} 2 \mathrm{~W}$ & $1.870(4)$ & $\mathrm{N} 4-\mathrm{C} 8$ & $1.396(3)$ \\
\hline Li1-O1W & $1.910(5)$ & $\mathrm{N} 4-\mathrm{H} 1 \mathrm{~N}$ & $0.84(3)$ \\
\hline Li1-O1 & $1.934(5)$ & $\mathrm{N} 4-\mathrm{H} 2 \mathrm{~N}$ & $0.93(3)$ \\
\hline $\mathrm{Li} 1-\mathrm{N} 2$ & $2.077(5)$ & $\mathrm{C} 2-\mathrm{C} 3$ & $1.370(4)$ \\
\hline $\mathrm{Li} 1-\mathrm{S} 1$ & $3.002(4)$ & $\mathrm{C} 2-\mathrm{H} 2$ & 0.9500 \\
\hline $\mathrm{S} 1-\mathrm{O} 2$ & $1.4533(17)$ & $\mathrm{C} 3-\mathrm{C} 4$ & $1.382(3)$ \\
\hline $\mathrm{S} 1-\mathrm{O} 1$ & $1.4612(16)$ & $\mathrm{C} 3-\mathrm{H} 3$ & 0.9500 \\
\hline $\mathrm{S} 1-\mathrm{N} 1$ & $1.5761(19)$ & $\mathrm{C} 4-\mathrm{H} 4$ & 0.9500 \\
\hline $\mathrm{S} 1-\mathrm{C} 5$ & $1.760(2)$ & $\mathrm{C} 5-\mathrm{C} 10$ & $1.385(3)$ \\
\hline $\mathrm{O} 1 \mathrm{~W}-\mathrm{H} 1 \mathrm{~W}$ & $0.875(12)$ & $\mathrm{C} 5-\mathrm{C} 6$ & $1.390(3)$ \\
\hline $\mathrm{O} 1 \mathrm{~W}-\mathrm{H} 2 \mathrm{~W}$ & $0.867(12)$ & $\mathrm{C} 6-\mathrm{C} 7$ & $1.378(3)$ \\
\hline $\mathrm{O} 2 \mathrm{~W}-\mathrm{H} 3 \mathrm{~W}$ & $0.865(12)$ & C6-H6 & 0.9500 \\
\hline $\mathrm{O} 2 \mathrm{~W}-\mathrm{H} 4 \mathrm{~W}$ & $0.863(12)$ & $\mathrm{C} 7-\mathrm{C} 8$ & $1.398(3)$ \\
\hline $\mathrm{N} 1-\mathrm{C} 1$ & $1.357(3)$ & $\mathrm{C} 7-\mathrm{H} 7$ & 0.9500 \\
\hline $\mathrm{N} 2-\mathrm{C} 2$ & $1.340(3)$ & $\mathrm{C} 8-\mathrm{C} 9$ & $1.397(3)$ \\
\hline $\mathrm{N} 2-\mathrm{C} 1$ & 1.355 & $\mathrm{C} 9-\mathrm{C} 10$ & $1.386(3)$ \\
\hline $\mathrm{N} 3-\mathrm{C} 4$ & $1.325(3)$ & C9- $\mathrm{H} 9$ & 0.9500 \\
\hline N3-C1 & $1.357(3)$ & $\mathrm{C} 10-\mathrm{H} 10$ & 0.9500 \\
\hline
\end{tabular}




\begin{tabular}{|c|c|c|c|}
\hline $\mathrm{O} 2 \mathrm{~W}-\mathrm{Li1}-\mathrm{O} 1 \mathrm{~W}$ & $101.5(2)$ & $\mathrm{C} 8-\mathrm{N} 4-\mathrm{H} 2 \mathrm{~N}$ & $111.7(17)$ \\
\hline $\mathrm{O} 2 \mathrm{~W}-\mathrm{Li} 1-\mathrm{O} 1$ & $126.9(2)$ & $\mathrm{H} 1 \mathrm{~N}-\mathrm{N} 4-\mathrm{H} 2 \mathrm{~N}$ & $113(3)$ \\
\hline $\mathrm{O} 1 \mathrm{~W}-\mathrm{Li} 1-\mathrm{O} 1$ & $108.5(2)$ & $\mathrm{N} 2-\mathrm{C} 1-\mathrm{N} 3$ & $123.4(2)$ \\
\hline $\mathrm{O} 2 \mathrm{~W}-\mathrm{Li} 1-\mathrm{N} 2$ & $107.1(2)$ & $\mathrm{N} 2-\mathrm{C} 1-\mathrm{N} 1$ & $122.3(2)$ \\
\hline $\mathrm{O} 1 \mathrm{~W}-\mathrm{Li} 1-\mathrm{N} 2$ & $124.1(2)$ & $\mathrm{N} 3-\mathrm{C} 1-\mathrm{N} 1$ & $114.3(2)$ \\
\hline $\mathrm{O} 1-\mathrm{Li} 1-\mathrm{N} 2$ & $90.91(18)$ & $\mathrm{N} 2-\mathrm{C} 2-\mathrm{C} 3$ & $123.3(2)$ \\
\hline $\mathrm{O} 2 \mathrm{~W}-\mathrm{Li} 1-\mathrm{S} 1$ & $125.5(2)$ & $\mathrm{N} 2-\mathrm{C} 2-\mathrm{H} 2$ & 118.4 \\
\hline $\mathrm{O} 1 \mathrm{~W}-\mathrm{Li} 1-\mathrm{S} 1$ & $126.84(19)$ & $\mathrm{C} 3-\mathrm{C} 2-\mathrm{H} 2$ & 118.4 \\
\hline $\mathrm{O} 1-\mathrm{Li} 1-\mathrm{S} 1$ & $23.87(8)$ & $\mathrm{C} 2-\mathrm{C} 3-\mathrm{C} 4$ & $115.9(2)$ \\
\hline $\mathrm{N} 2-\mathrm{Li} 1-\mathrm{S} 1$ & $68.13(13)$ & $\mathrm{C} 2-\mathrm{C} 3-\mathrm{H} 3$ & 122.0 \\
\hline $\mathrm{O} 2-\mathrm{S} 1-\mathrm{O} 1$ & $113.09(10)$ & $\mathrm{C} 4-\mathrm{C} 3-\mathrm{H} 3$ & 122.0 \\
\hline $\mathrm{O} 2-\mathrm{S} 1-\mathrm{N} 1$ & $106.15(10)$ & $\mathrm{N} 3-\mathrm{C} 4-\mathrm{C} 3$ & $123.2(2)$ \\
\hline $\mathrm{O} 1-\mathrm{S} 1-\mathrm{N} 1$ & $115.22(10)$ & $\mathrm{N} 3-\mathrm{C} 4-\mathrm{H} 4$ & 118.4 \\
\hline $\mathrm{O} 2-\mathrm{S} 1-\mathrm{C} 5$ & $107.73(10)$ & $\mathrm{C} 3-\mathrm{C} 4-\mathrm{H} 4$ & 118.4 \\
\hline $\mathrm{O} 1-\mathrm{S} 1-\mathrm{C} 5$ & $107.16(10)$ & $\mathrm{C} 10-\mathrm{C} 5-\mathrm{C} 6$ & $120.4(2)$ \\
\hline $\mathrm{N} 1-\mathrm{S} 1-\mathrm{C} 5$ & $107.14(10)$ & $\mathrm{C} 10-\mathrm{C} 5-\mathrm{S} 1$ & $120.99(18)$ \\
\hline $\mathrm{O} 2-\mathrm{S} 1-\mathrm{Li} 1$ & $144.30(11)$ & $\mathrm{C} 6-\mathrm{C} 5-\mathrm{S} 1$ & $118.58(18)$ \\
\hline $\mathrm{O} 1-\mathrm{S} 1-\mathrm{Li} 1$ & $32.38(11)$ & $\mathrm{C} 7-\mathrm{C} 6-\mathrm{C} 5$ & $119.7(2)$ \\
\hline $\mathrm{N} 1-\mathrm{S} 1-\mathrm{Li} 1$ & $91.02(11)$ & $\mathrm{C} 7-\mathrm{C} 6-\mathrm{H} 6$ & 120.1 \\
\hline $\mathrm{C} 5-\mathrm{S} 1-\mathrm{Li} 1$ & $96.20(11)$ & $\mathrm{C} 5-\mathrm{C} 6-\mathrm{H} 6$ & 120.1 \\
\hline $\mathrm{S} 1-\mathrm{O} 1-\mathrm{Li} 1$ & $123.74(16)$ & $\mathrm{C} 6-\mathrm{C} 7-\mathrm{C} 8$ & $120.7(2)$ \\
\hline $\mathrm{Li1}-\mathrm{O} 1 \mathrm{~W}-\mathrm{H} 1 \mathrm{~W}$ & $124(2)$ & $\mathrm{C} 6-\mathrm{C} 7-\mathrm{H} 7$ & 119.7 \\
\hline $\mathrm{Li} 1-\mathrm{O} 1 \mathrm{~W}-\mathrm{H} 2 \mathrm{~W}$ & $117(2)$ & $\mathrm{C} 8-\mathrm{C} 7-\mathrm{H} 7$ & 119.7 \\
\hline $\mathrm{H} 1 \mathrm{~W}-\mathrm{O} 1 \mathrm{~W}-\mathrm{H} 2 \mathrm{~W}$ & $101(2)$ & $\mathrm{N} 4-\mathrm{C} 8-\mathrm{C} 9$ & $120.8(2)$ \\
\hline $\mathrm{Li} 1-\mathrm{O} 2 \mathrm{~W}-\mathrm{H} 3 \mathrm{~W}$ & $123(3)$ & $\mathrm{N} 4-\mathrm{C} 8-\mathrm{C} 7$ & $120.1(2)$ \\
\hline $\mathrm{Li1}-\mathrm{O} 2 \mathrm{~W}-\mathrm{H} 4 \mathrm{~W}$ & $134(3)$ & $\mathrm{C} 9-\mathrm{C} 8-\mathrm{C} 7$ & $119.0(2)$ \\
\hline $\mathrm{H} 3 \mathrm{~W}-\mathrm{O} 2 \mathrm{~W}-\mathrm{H} 4 \mathrm{~W}$ & $104(2)$ & $\mathrm{C} 10-\mathrm{C} 9-\mathrm{C} 8$ & $120.4(2)$ \\
\hline $\mathrm{C} 1-\mathrm{N} 1-\mathrm{S} 1$ & $122.79(16)$ & $\mathrm{C} 10-\mathrm{C} 9-\mathrm{H} 9$ & 119.8 \\
\hline $\mathrm{C} 2-\mathrm{N} 2-\mathrm{C} 1$ & $116.9(2)$ & $\mathrm{C} 8-\mathrm{C} 9-\mathrm{H} 9$ & 119.8 \\
\hline $\mathrm{C} 2-\mathrm{N} 2-\mathrm{Li} 1$ & $112.7(2)$ & $\mathrm{C} 5-\mathrm{C} 10-\mathrm{C} 9$ & $119.7(2)$ \\
\hline $\mathrm{C} 1-\mathrm{N} 2-\mathrm{Li} 1$ & $127.0(2)$ & $\mathrm{C} 5-\mathrm{C} 10-\mathrm{H} 10$ & 120.1 \\
\hline $\mathrm{C} 4-\mathrm{N} 3-\mathrm{C} 1$ & $117.3(2)$ & $\mathrm{C} 9-\mathrm{C} 10-\mathrm{H} 10$ & 120.1 \\
\hline $\mathrm{C} 8-\mathrm{N} 4-\mathrm{H} 1 \mathrm{~N}$ & $116(2)$ & & \\
\hline $\mathrm{O} 2-\mathrm{S} 1-\mathrm{O} 1-\mathrm{Li} 1$ & $-167.45(18)$ & $\mathrm{C} 2-\mathrm{C} 3-\mathrm{C} 4-\mathrm{N} 3$ & $-1.0(4)$ \\
\hline $\mathrm{N} 1-\mathrm{S} 1-\mathrm{O} 1-\mathrm{Li} 1$ & $-45.1(2)$ & $\mathrm{O} 2-\mathrm{S} 1-\mathrm{C} 5-\mathrm{C} 10$ & $-123.58(19)$ \\
\hline $\mathrm{C} 5-\mathrm{S} 1-\mathrm{O} 1-\mathrm{Li} 1$ & $74.0(2)$ & $\mathrm{O} 1-\mathrm{S} 1-\mathrm{C} 5-\mathrm{C} 10$ & $-1.6(2)$ \\
\hline $\mathrm{O} 2-\mathrm{S} 1-\mathrm{N} 1-\mathrm{C} 1$ & $169.98(18)$ & $\mathrm{N} 1-\mathrm{S} 1-\mathrm{C} 5-\mathrm{C} 10$ & $122.58(19)$ \\
\hline $\mathrm{O} 1-\mathrm{S} 1-\mathrm{N} 1-\mathrm{C} 1$ & $44.0(2)$ & $\mathrm{Li} 1-\mathrm{S} 1-\mathrm{C} 5-\mathrm{C} 10$ & $29.6(2)$ \\
\hline $\mathrm{C} 5-\mathrm{S} 1-\mathrm{N} 1-\mathrm{C} 1$ & $-75.1(2)$ & $\mathrm{O} 2-\mathrm{S} 1-\mathrm{C} 5-\mathrm{C} 6$ & $56.4(2)$ \\
\hline $\mathrm{Li} 1-\mathrm{S} 1-\mathrm{N} 1-\mathrm{C} 1$ & $21.7(2)$ & $\mathrm{O} 1-\mathrm{S} 1-\mathrm{C} 5-\mathrm{C} 6$ & $178.37(18)$ \\
\hline $\mathrm{C} 2-\mathrm{N} 2-\mathrm{C} 1-\mathrm{N} 3$ & $-1.4(3)$ & $\mathrm{N} 1-\mathrm{S} 1-\mathrm{C} 5-\mathrm{C} 6$ & $-57.4(2)$ \\
\hline $\mathrm{Li} 1-\mathrm{N} 2-\mathrm{C} 1-\mathrm{N} 3$ & $156.1(2)$ & $\mathrm{Li} 1-\mathrm{S} 1-\mathrm{C} 5-\mathrm{C} 6$ & $-150.4(2)$ \\
\hline $\mathrm{C} 2-\mathrm{N} 2-\mathrm{C} 1-\mathrm{N} 1$ & $179.3(2)$ & $\mathrm{C} 10-\mathrm{C} 5-\mathrm{C} 6-\mathrm{C} 7$ & $-1.7(4)$ \\
\hline $\mathrm{Li} 1-\mathrm{N} 2-\mathrm{C} 1-\mathrm{N} 1$ & $-23.3(4)$ & $\mathrm{S} 1-\mathrm{C} 5-\mathrm{C} 6-\mathrm{C} 7$ & $178.34(19)$ \\
\hline $\mathrm{C} 4-\mathrm{N} 3-\mathrm{C} 1-\mathrm{N} 2$ & $0.1(3)$ & $\mathrm{C} 5-\mathrm{C} 6-\mathrm{C} 7-\mathrm{C} 8$ & $0.1(4)$ \\
\hline $\mathrm{C} 4-\mathrm{N} 3-\mathrm{C} 1-\mathrm{N} 1$ & $179.5(2)$ & $\mathrm{C} 6-\mathrm{C} 7-\mathrm{C} 8-\mathrm{N} 4$ & $-174.7(2)$ \\
\hline $\mathrm{S} 1-\mathrm{N} 1-\mathrm{C} 1-\mathrm{N} 2$ & $-8.8(3)$ & $\mathrm{C} 6-\mathrm{C} 7-\mathrm{C} 8-\mathrm{C} 9$ & $1.8(4)$ \\
\hline
\end{tabular}



$\mathrm{S} 1-\mathrm{N} 1-\mathrm{C} 1-\mathrm{N} 3$
$171.78(16)$
$1.5(4)$
$\mathrm{C} 1-\mathrm{N} 2-\mathrm{C} 2-\mathrm{C} 3$
$-159.1(3)$
$\mathrm{N} 2-\mathrm{C} 2-\mathrm{C} 3-\mathrm{C} 4$
$-0.4(4)$
$1.2(4)$

$174.3(2)$

$-2.1(3)$

$1.4(3)$

$-178.66(17)$

$0.5(3)$

Hydrogen-bond geometry $\left(A,{ }^{\circ}\right)$

\begin{tabular}{lllll}
\hline$D-\mathrm{H} \cdots A$ & $D-\mathrm{H}$ & $\mathrm{H} \cdots A$ & $D \cdots A$ & $D-\mathrm{H} \cdots A$ \\
\hline $\mathrm{O} 1 W-\mathrm{H} 1 W \cdots \mathrm{O} 2^{\mathrm{i}}$ & $0.88(1)$ & $2.05(2)$ & $2.881(2)$ & $158(3)$ \\
$\mathrm{O} 2 W-\mathrm{H} 3 W \cdots \mathrm{N} 1^{\text {ii }}$ & $0.87(1)$ & $1.95(2)$ & $2.805(3)$ & $170(4)$ \\
$\mathrm{O} 1 W-\mathrm{H} 2 W \cdots \mathrm{N} 3^{\text {ii }}$ & $0.87(1)$ & $1.97(1)$ & $2.837(3)$ & $178(3)$ \\
$\mathrm{O} 2 W-\mathrm{H} 4 W \cdots \mathrm{N} 4^{\text {iii }}$ & $0.86(1)$ & $2.01(1)$ & $2.866(3)$ & $174(4)$ \\
$\mathrm{N} 4-\mathrm{H} 1 N \cdots \mathrm{O} 2^{\text {iv }}$ & $0.84(3)$ & $2.40(3)$ & $3.070(3)$ & $137(3)$ \\
\hline
\end{tabular}

Symmetry codes: (i) $x,-y+1, z-1 / 2$; (ii) $x+1 / 2,-y+1 / 2, z-1 / 2$; (iii) $x,-y, z-1 / 2$; (iv) $x+1 / 2, y-1 / 2, z$.

(II)

\section{Crystal data}

\section{$\mathrm{C}_{10} \mathrm{H}_{9} \mathrm{~N}_{4} \mathrm{NaO}_{2} \mathrm{~S}$}

$M_{r}=272.26$

Monoclinic, $P 2_{1} / c$

$a=5.9010(11) \AA$

$b=10.534(4) \AA$

$c=18.389(3) \AA$

$\beta=94.216(15)^{\circ}$

$V=1140.0(5) \AA^{3}$

$Z=4$

\section{Data collection}

Oxford Diffraction Xcalibur E diffractometer

Radiation source: sealed tube $\omega$ scans

\section{Refinement}

Refinement on $F^{2}$ Least-squares matrix: full $R\left[F^{2}>2 \sigma\left(F^{2}\right)\right]=0.038$ $w R\left(F^{2}\right)=0.088$
$F(000)=560$

$D_{\mathrm{x}}=1.586 \mathrm{Mg} \mathrm{m}^{-3}$

Mo $K \alpha$ radiation, $\lambda=0.71073 \AA$

Cell parameters from 2254 reflections

$\theta=3.3-29.4^{\circ}$

$\mu=0.32 \mathrm{~mm}^{-1}$

$T=123 \mathrm{~K}$

Rod, colourless

$0.35 \times 0.12 \times 0.08 \mathrm{~mm}$

Absorption correction: multi-scan

CrysAlis PRO, Oxford Diffraction Ltd., Version

1.171.34.40 (release 27-08-2010 CrysAlis171

.NET) (compiled Aug 27 2010,11:50:40)

Empirical absorption correction using spherical harmonics, implemented in SCALE3

ABSPACK scaling algorithm.

$T_{\min }=0.961, T_{\max }=1.000$

5903 measured reflections

2786 independent reflections

2351 reflections with $I>2 \sigma(I)$

$R_{\text {int }}=0.029$

$\theta_{\max }=29.5^{\circ}, \theta_{\min }=3.5^{\circ}$

$h=-7 \rightarrow 7$

$k=-12 \rightarrow 14$

$l=-24 \rightarrow 24$

$S=1.06$

2786 reflections

171 parameters

0 restraints 
Hydrogen site location: mixed

$\mathrm{H}$ atoms treated by a mixture of independent and constrained refinement

$$
\begin{aligned}
& w=1 /\left[\sigma^{2}\left(F_{\mathrm{o}}^{2}\right)+(0.0278 P)^{2}+0.6871 P\right] \\
& \text { where } P=\left(F_{\mathrm{o}}^{2}+2 F_{\mathrm{c}}^{2}\right) / 3 \\
& (\Delta / \sigma)_{\max }<0.001 \\
& \Delta \rho_{\max }=0.43 \mathrm{e} \AA^{-3} \\
& \Delta \rho_{\min }=-0.46 \mathrm{e} \AA^{-3}
\end{aligned}
$$

\section{Special details}

Geometry. All e.s.d.'s (except the e.s.d. in the dihedral angle between two 1.s. planes) are estimated using the full covariance matrix. The cell e.s.d.'s are taken into account individually in the estimation of e.s.d.'s in distances, angles and torsion angles; correlations between e.s.d.'s in cell parameters are only used when they are defined by crystal symmetry. An approximate (isotropic) treatment of cell e.s.d.'s is used for estimating e.s.d.'s involving 1.s. planes.

Fractional atomic coordinates and isotropic or equivalent isotropic displacement parameters $\left(\AA^{2}\right)$

\begin{tabular}{lllll}
\hline & $x$ & $y$ & $z$ & $U_{\text {iso }} / U_{\text {eq }}$ \\
\hline Na1 & $0.41732(11)$ & $-0.15699(7)$ & $0.03430(4)$ & $0.01483(18)$ \\
S1 & $0.13569(7)$ & $0.11080(4)$ & $0.07836(2)$ & $0.01030(12)$ \\
O1 & $0.34050(19)$ & $0.07207(12)$ & $0.04443(7)$ & $0.0130(3)$ \\
O2 & $0.0133(2)$ & $0.00644(12)$ & $0.10838(7)$ & $0.0152(3)$ \\
N1 & $-0.0383(2)$ & $0.18774(14)$ & $0.02732(8)$ & $0.0121(3)$ \\
N2 & $0.2445(2)$ & $0.32085(15)$ & $-0.01969(9)$ & $0.0154(3)$ \\
N3 & $-0.1495(2)$ & $0.33011(16)$ & $-0.06053(9)$ & $0.0155(3)$ \\
N4 & $0.4138(3)$ & $0.41926(17)$ & $0.34001(9)$ & $0.0182(4)$ \\
C1 & $0.0271(3)$ & $0.28102(17)$ & $-0.01786(10)$ & $0.0122(4)$ \\
C2 & $0.2788(3)$ & $0.42162(19)$ & $-0.06182(11)$ & $0.0197(4)$ \\
H2 & 0.4295 & 0.4527 & -0.0633 & $0.024^{*}$ \\
C3 & $0.1082(3)$ & $0.4826(2)$ & $-0.10308(12)$ & $0.0224(4)$ \\
H3 & 0.1351 & 0.5566 & -0.1307 & $0.027^{*}$ \\
C4 & $-0.1042(3)$ & $0.4292(2)$ & $-0.10165(11)$ & $0.0198(4)$ \\
H4 & -0.2252 & 0.4653 & -0.1318 & $0.024^{*}$ \\
C5 & $0.2237(3)$ & $0.20545(17)$ & $0.15397(10)$ & $0.0121(4)$ \\
C6 & $0.0767(3)$ & $0.21945(18)$ & $0.20992(10)$ & $0.0154(4)$ \\
H6 & -0.0670 & 0.1783 & 0.2059 & $0.019^{*}$ \\
C7 & $0.1368(3)$ & $0.29163(19)$ & $0.27051(10)$ & $0.0162(4)$ \\
H7 & 0.0348 & 0.3008 & 0.3078 & $0.019^{*}$ \\
C8 & $0.3482(3)$ & $0.35145(18)$ & $0.27712(10)$ & $0.0141(4)$ \\
C9 & $0.4953(3)$ & $0.33673(19)$ & $0.22135(11)$ & $0.0177(4)$ \\
H9 & 0.6399 & 0.3768 & 0.2257 & $0.021^{*}$ \\
C10 & $0.4342(3)$ & $0.26494(19)$ & $0.15998(10)$ & $0.0160(4)$ \\
H10 & 0.5352 & 0.2565 & 0.1224 & $0.019^{*}$ \\
H1N & $0.301(4)$ & $0.454(2)$ & $0.3602(13)$ & $0.023(6)^{*}$ \\
H2N & $0.528(4)$ & $0.476(2)$ & $0.3346(13)$ & $0.029(6)^{*}$ \\
& & & &
\end{tabular}

Atomic displacement parameters $\left(\AA^{2}\right)$

\begin{tabular}{lllllll}
\hline & $U^{11}$ & $U^{22}$ & $U^{33}$ & $U^{12}$ & $U^{13}$ & $U^{23}$ \\
\hline $\mathrm{Na} 1$ & $0.0126(3)$ & $0.0166(4)$ & $0.0152(4)$ & $-0.0003(3)$ & $0.0003(3)$ & $0.0012(3)$ \\
$\mathrm{S} 1$ & $0.0107(2)$ & $0.0106(2)$ & $0.0097(2)$ & $0.00025(15)$ & $0.00075(15)$ & $-0.00029(17)$ \\
$\mathrm{O} 1$ & $0.0130(6)$ & $0.0136(6)$ & $0.0126(6)$ & $0.0030(5)$ & $0.0020(5)$ & $-0.0009(5)$
\end{tabular}




$\begin{array}{lllllll}\mathrm{O} 2 & 0.0180(6) & 0.0121(6) & 0.0159(7) & -0.0030(5) & 0.0026(5) & 0.0004(6) \\ \mathrm{N} 1 & 0.0107(7) & 0.0137(8) & 0.0118(8) & 0.0004(6) & -0.0005(5) & 0.0013(6) \\ \mathrm{N} 2 & 0.0119(7) & 0.0156(8) & 0.0188(8) & -0.0006(6) & 0.0019(6) & 0.0007(7) \\ \mathrm{N} 3 & 0.0126(7) & 0.0173(8) & 0.0163(8) & 0.0029(6) & -0.0002(6) & 0.0032(7) \\ \mathrm{N} 4 & 0.0157(8) & 0.0218(9) & 0.0168(9) & -0.0016(7) & -0.0016(6) & -0.0077(7) \\ \mathrm{C} 1 & 0.0116(8) & 0.0136(9) & 0.0114(9) & 0.0017(7) & 0.0013(6) & -0.0022(7) \\ \mathrm{C} 2 & 0.0165(9) & 0.0183(10) & 0.0247(11) & -0.0013(7) & 0.0052(7) & 0.0027(9) \\ \mathrm{C} 3 & 0.0243(10) & 0.0171(10) & 0.0267(12) & 0.0027(8) & 0.0084(8) & 0.0075(9) \\ \mathrm{C} 4 & 0.0182(9) & 0.0212(10) & 0.0203(10) & 0.0067(8) & 0.0023(7) & 0.0053(9) \\ \mathrm{C} 5 & 0.0123(8) & 0.0131(9) & 0.0106(9) & 0.0017(7) & -0.0014(6) & 0.0002(7) \\ \mathrm{C} 6 & 0.0125(8) & 0.0194(10) & 0.0143(9) & -0.0019(7) & 0.0011(7) & -0.0023(8) \\ \mathrm{C} 7 & 0.0132(8) & 0.0212(10) & 0.0145(9) & -0.0003(7) & 0.0027(7) & -0.0021(8) \\ \mathrm{C} 8 & 0.0146(8) & 0.0143(9) & 0.0129(9) & 0.0013(7) & -0.0024(7) & -0.0014(8) \\ \text { C9 } & 0.0131(8) & 0.0223(10) & 0.0174(10) & -0.0041(7) & -0.0010(7) & -0.0024(9) \\ \text { C10 } & 0.0132(8) & 0.0204(10) & 0.0148(9) & -0.0012(7) & 0.0031(7) & -0.0011(8)\end{array}$

Geometric parameters $(\AA, \stackrel{o}{)})$

\begin{tabular}{|c|c|c|c|}
\hline $\mathrm{Na} 1-\mathrm{O} 1^{\mathrm{i}}$ & $2.2897(15)$ & $\mathrm{N} 4-\mathrm{C} 8$ & $1.390(2)$ \\
\hline $\mathrm{Na} 1-\mathrm{N} 1^{\mathrm{ii}}$ & $2.4533(15)$ & $\mathrm{N} 4-\mathrm{Na} 1^{\mathrm{iv}}$ & $2.5772(19)$ \\
\hline $\mathrm{Na} 1-\mathrm{O} 1$ & 2.4647 (17) & $\mathrm{N} 4-\mathrm{H} 1 \mathrm{~N}$ & $0.86(2)$ \\
\hline $\mathrm{Na} 1-\mathrm{N} 3^{\mathrm{ii}}$ & $2.4836(18)$ & $\mathrm{N} 4-\mathrm{H} 2 \mathrm{~N}$ & $0.91(2)$ \\
\hline $\mathrm{Na} 1-\mathrm{N} 4^{\mathrm{iii}}$ & $2.5771(19)$ & $\mathrm{C} 1-\mathrm{Na} 1^{\mathrm{ii}}$ & $2.9255(19)$ \\
\hline $\mathrm{Na} 1-\mathrm{N} 2^{\mathrm{i}}$ & $2.6670(17)$ & $\mathrm{C} 2-\mathrm{C} 3$ & $1.375(3)$ \\
\hline $\mathrm{Na} 1-\mathrm{C} 1^{\mathrm{ii}}$ & $2.9255(19)$ & $\mathrm{C} 2-\mathrm{H} 2$ & 0.9500 \\
\hline $\mathrm{Na} 1-\mathrm{Na} 1^{\mathrm{i}}$ & 3.6964 (19) & $\mathrm{C} 3-\mathrm{C} 4$ & $1.376(3)$ \\
\hline $\mathrm{S} 1-\mathrm{O} 2$ & 1.4470 (14) & $\mathrm{C} 3-\mathrm{H} 3$ & 0.9500 \\
\hline $\mathrm{S} 1-\mathrm{O} 1$ & $1.4585(13)$ & $\mathrm{C} 4-\mathrm{H} 4$ & 0.9500 \\
\hline $\mathrm{S} 1-\mathrm{N} 1$ & $1.5652(15)$ & $\mathrm{C} 5-\mathrm{C} 10$ & $1.388(2)$ \\
\hline $\mathrm{S} 1-\mathrm{C} 5$ & $1.7582(18)$ & $\mathrm{C} 5-\mathrm{C} 6$ & $1.402(2)$ \\
\hline $\mathrm{O} 1-\mathrm{Na} 1^{\mathrm{i}}$ & $2.2897(15)$ & $\mathrm{C} 6-\mathrm{C} 7$ & $1.374(3)$ \\
\hline $\mathrm{N} 1-\mathrm{C} 1$ & $1.361(2)$ & $\mathrm{C} 6-\mathrm{H} 6$ & 0.9500 \\
\hline $\mathrm{N} 1-\mathrm{Na} 1^{\mathrm{ii}}$ & $2.4533(15)$ & $\mathrm{C} 7-\mathrm{C} 8$ & $1.395(2)$ \\
\hline $\mathrm{N} 2-\mathrm{C} 2$ & $1.338(3)$ & $\mathrm{C} 7-\mathrm{H} 7$ & 0.9500 \\
\hline $\mathrm{N} 2-\mathrm{C} 1$ & $1.353(2)$ & $\mathrm{C} 8-\mathrm{C} 9$ & $1.400(3)$ \\
\hline $\mathrm{N} 2-\mathrm{Na} 1^{\mathrm{i}}$ & $2.6670(17)$ & $\mathrm{C} 9-\mathrm{C} 10$ & $1.384(3)$ \\
\hline N3-C4 & $1.328(3)$ & $\mathrm{C} 9-\mathrm{H} 9$ & 0.9500 \\
\hline $\mathrm{N} 3-\mathrm{C} 1$ & $1.360(2)$ & $\mathrm{C} 10-\mathrm{H} 10$ & 0.9500 \\
\hline $\mathrm{N} 3-\mathrm{Na} 1^{\mathrm{ii}}$ & $2.4836(18)$ & & \\
\hline $\mathrm{O} 1{ }^{\mathrm{i}}-\mathrm{Na} 1-\mathrm{N} 1^{\mathrm{ii}}$ & $110.47(5)$ & $\mathrm{C} 4-\mathrm{N} 3-\mathrm{C} 1$ & $116.73(16)$ \\
\hline $\mathrm{O} 1-\mathrm{Na} 1-\mathrm{O} 1$ & $78.00(5)$ & $\mathrm{C} 4-\mathrm{N} 3-\mathrm{Na} 1^{\mathrm{ii}}$ & $147.74(13)$ \\
\hline $\mathrm{N} 11^{\mathrm{ii}}-\mathrm{Na} 1-\mathrm{O} 1$ & $89.87(5)$ & $\mathrm{C} 1-\mathrm{N} 3-\mathrm{Na} 1^{\mathrm{ii}}$ & $94.60(11)$ \\
\hline 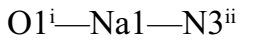 & $147.71(6)$ & $\mathrm{C} 8-\mathrm{N} 4-\mathrm{Na} 1^{\mathrm{iv}}$ & $130.68(13)$ \\
\hline 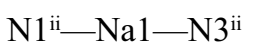 & $54.64(5)$ & $\mathrm{C} 8-\mathrm{N} 4-\mathrm{H} 1 \mathrm{~N}$ & $113.5(15)$ \\
\hline $\mathrm{O} 1-\mathrm{Na} 1-\mathrm{N} 3^{\mathrm{ii}}$ & $125.52(6)$ & $\mathrm{Na} 1^{\mathrm{iv}}-\mathrm{N} 4-\mathrm{H} 1 \mathrm{~N}$ & $90.2(15)$ \\
\hline $\mathrm{O} 1^{\mathrm{i}}-\mathrm{Na} 1-\mathrm{N} 4^{\mathrm{iii}}$ & $103.13(6)$ & $\mathrm{C} 8-\mathrm{N} 4-\mathrm{H} 2 \mathrm{~N}$ & $114.1(15)$ \\
\hline $\mathrm{N} 1^{\mathrm{ii}}-\mathrm{Na} 1-\mathrm{N} 4^{\mathrm{iii}}$ & $137.28(6)$ & $\mathrm{Na} 1^{\mathrm{iv}}-\mathrm{N} 4-\mathrm{H} 2 \mathrm{~N}$ & $93.5(15)$ \\
\hline
\end{tabular}


$\mathrm{O} 1-\mathrm{Na} 1-\mathrm{N} 4^{\mathrm{iii}}$

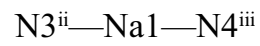

$\mathrm{O} 1^{\mathrm{i}}-\mathrm{Na} 1-\mathrm{N} 2^{\mathrm{i}}$

$\mathrm{N} 1^{\mathrm{ii}}-\mathrm{Na} 1-\mathrm{N} 2^{\mathrm{i}}$

$\mathrm{O} 1-\mathrm{Na} 1-\mathrm{N} 2^{\mathrm{i}}$

$\mathrm{N} 3^{3 i}-\mathrm{Na} 1-\mathrm{N} 2^{\mathrm{i}}$

$\mathrm{N} 4^{i i i}-\mathrm{Na} 1-\mathrm{N} 2^{\mathrm{i}}$

$\mathrm{O} 1^{\mathrm{i}}-\mathrm{Na} 1-\mathrm{Cl}^{\mathrm{ii}}$

$\mathrm{N} 1^{\mathrm{ii}}-\mathrm{Na} 1-\mathrm{C}^{\mathrm{ii}}$

$\mathrm{O} 1-\mathrm{Na} 1-\mathrm{C}{ }^{\mathrm{ii}}$

$\mathrm{N} 3^{3 i}-\mathrm{Na} 1-\mathrm{C}^{\mathrm{ii}}$

$\mathrm{N} 4{ }^{i i i}-\mathrm{Na} 1-\mathrm{C} 1^{\mathrm{ii}}$

$\mathrm{N} 2{ }^{\mathrm{i}}-\mathrm{Na} 1-\mathrm{C} 1^{\mathrm{ii}}$

$\mathrm{O} 1^{\mathrm{i}}-\mathrm{Na} 1-\mathrm{Na} 1^{\mathrm{i}}$

$\mathrm{N} 1{ }^{1 i}-\mathrm{Na} 1-\mathrm{Na} 1^{\mathrm{i}}$

$\mathrm{O} 1-\mathrm{Na} 1-\mathrm{Na} 1^{\mathrm{i}}$

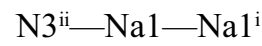

$\mathrm{N} 4{ }^{\mathrm{iii}}-\mathrm{Na} 1-\mathrm{Na}{ }^{\mathrm{i}}$

$\mathrm{N} 2^{\mathrm{i}}-\mathrm{Na} 1-\mathrm{Na} 1^{\mathrm{i}}$

$\mathrm{C} 1^{\mathrm{ii}}-\mathrm{Na} 1-\mathrm{Na} 1^{\mathrm{i}}$

$\mathrm{O} 2-\mathrm{S} 1-\mathrm{O} 1$

$\mathrm{O} 2-\mathrm{S} 1-\mathrm{N} 1$

$\mathrm{O} 1-\mathrm{S} 1-\mathrm{N} 1$

$\mathrm{O} 2-\mathrm{S} 1-\mathrm{C} 5$

$\mathrm{O} 1-\mathrm{S} 1-\mathrm{C} 5$

$\mathrm{N} 1-\mathrm{S} 1-\mathrm{C} 5$

$\mathrm{S} 1-\mathrm{O} 1-\mathrm{Na} 1^{\mathrm{i}}$

$\mathrm{S} 1-\mathrm{O} 1-\mathrm{Na} 1$

$\mathrm{Na} 1-\mathrm{O} 1-\mathrm{Na} 1$

$\mathrm{C} 1-\mathrm{N} 1-\mathrm{S} 1$

$\mathrm{C} 1-\mathrm{N} 1-\mathrm{Na} 1^{\mathrm{ii}}$

$\mathrm{S} 1-\mathrm{N} 1-\mathrm{Na} 1^{\mathrm{ii}}$

$\mathrm{C} 2-\mathrm{N} 2-\mathrm{C} 1$

$\mathrm{C} 2-\mathrm{N} 2-\mathrm{Na}^{\mathrm{i}}$

$\mathrm{C} 1-\mathrm{N} 2-\mathrm{Na} 1^{\mathrm{i}}$

$\mathrm{O} 2-\mathrm{S} 1-\mathrm{O} 1-\mathrm{Na} 1^{\mathrm{i}}$

$\mathrm{N} 1-\mathrm{S} 1-\mathrm{O} 1-\mathrm{Na}^{\mathrm{i}}$

$\mathrm{C} 5-\mathrm{S} 1-\mathrm{O} 1-\mathrm{Na} 1^{\mathrm{i}}$

$\mathrm{O} 2-\mathrm{S} 1-\mathrm{O} 1-\mathrm{Na} 1$

$\mathrm{N} 1-\mathrm{S} 1-\mathrm{O} 1-\mathrm{Na} 1$

$\mathrm{C} 5-\mathrm{S} 1-\mathrm{O} 1-\mathrm{Na} 1$

$\mathrm{O} 2-\mathrm{S} 1-\mathrm{N} 1-\mathrm{C} 1$

$\mathrm{O} 1-\mathrm{S} 1-\mathrm{N} 1-\mathrm{C} 1$

$\mathrm{C} 5-\mathrm{S} 1-\mathrm{N} 1-\mathrm{C} 1$

$\mathrm{O} 2-\mathrm{S} 1-\mathrm{N} 1-\mathrm{Na} 1^{\mathrm{ii}}$

$\mathrm{O} 1-\mathrm{S} 1-\mathrm{N} 1-\mathrm{Na} 1^{\mathrm{ii}}$

$\mathrm{C} 5-\mathrm{S} 1-\mathrm{N} 1-\mathrm{Na} 1^{\mathrm{ii}}$
$71.83(5)$

$105.32(6)$

$71.51(5)$

$122.11(6)$

$141.66(5)$

$92.06(6)$

$92.82(6)$

$134.88(6)$

$27.56(5)$

$106.06(5)$

$27.60(5)$

121.09 (6)

111.85 (6)

40.71 (3)

$102.42(5)$

$37.29(4)$

$155.65(5)$

$86.15(5)$

$109.06(5)$

$128.42(5)$

$113.78(8)$

$107.35(8)$

$114.59(8)$

$104.83(8)$

$106.92(8)$

$108.86(8)$

$137.43(8)$

117.97 (7)

$102.00(5)$

$122.48(12)$

$95.92(10)$

138.08 (9)

$116.40(15)$

$107.99(12)$

121.33 (12)

$163.45(10)$

39.38 (14)

$-81.32(12)$

$5.61(10)$

$-118.45(8)$

$120.84(8)$

$-170.13(14)$

-42.71 (17)

$76.92(16)$

$-17.00(15)$

110.42 (13)

-129.95 (13)
$\mathrm{H} 1 \mathrm{~N}-\mathrm{N} 4-\mathrm{H} 2 \mathrm{~N}$

112 (2)

$\mathrm{N} 2-\mathrm{C} 1-\mathrm{N} 3$

$\mathrm{N} 2-\mathrm{C} 1-\mathrm{N} 1$

N3- $1-\mathrm{N} 1$

$\mathrm{N} 2-\mathrm{C} 1-\mathrm{Na} 1^{\mathrm{ii}}$

N3- $1-\mathrm{Na}^{1 i}$

$\mathrm{N} 1-\mathrm{C} 1-\mathrm{Na} 1^{1 \mathrm{i}}$

$\mathrm{N} 2-\mathrm{C} 2-\mathrm{C} 3$

$\mathrm{N} 2-\mathrm{C} 2-\mathrm{H} 2$

$\mathrm{C} 3-\mathrm{C} 2-\mathrm{H} 2$

$\mathrm{C} 2-\mathrm{C} 3-\mathrm{C} 4$

$\mathrm{C} 2-\mathrm{C} 3-\mathrm{H} 3$

$\mathrm{C} 4-\mathrm{C} 3-\mathrm{H} 3$

$\mathrm{N} 3-\mathrm{C} 4-\mathrm{C} 3$

$\mathrm{N} 3-\mathrm{C} 4-\mathrm{H} 4$

$\mathrm{C} 3-\mathrm{C} 4-\mathrm{H} 4$

$\mathrm{C} 10-\mathrm{C} 5-\mathrm{C} 6$

$\mathrm{C} 10-\mathrm{C} 5-\mathrm{S} 1$

C6- C5-S1

$\mathrm{C} 7-\mathrm{C} 6-\mathrm{C} 5$

$\mathrm{C} 7-\mathrm{C} 6-\mathrm{H} 6$

$\mathrm{C} 5-\mathrm{C} 6-\mathrm{H} 6$

$\mathrm{C} 6-\mathrm{C} 7-\mathrm{C} 8$

$\mathrm{C} 6-\mathrm{C} 7-\mathrm{H} 7$

$\mathrm{C} 8-\mathrm{C} 7-\mathrm{H} 7$

$\mathrm{N} 4-\mathrm{C} 8-\mathrm{C} 7$

$\mathrm{N} 4-\mathrm{C} 8-\mathrm{C} 9$

$\mathrm{C} 7-\mathrm{C} 8-\mathrm{C} 9$

$\mathrm{C} 10-\mathrm{C} 9-\mathrm{C} 8$

$\mathrm{C} 10-\mathrm{C} 9-\mathrm{H} 9$

C8-C9-H9

C9- $\mathrm{C} 10-\mathrm{C} 5$

$\mathrm{C} 9-\mathrm{C} 10-\mathrm{H} 10$

$\mathrm{C} 5-\mathrm{C} 10-\mathrm{H} 10$

$\mathrm{Na} 1^{\mathrm{ii}}-\mathrm{N} 1-\mathrm{C} 1-\mathrm{N} 3$

$\mathrm{S} 1-\mathrm{N} 1-\mathrm{C} 1-\mathrm{Na} 1^{\mathrm{ii}}$

$\mathrm{C} 1-\mathrm{N} 2-\mathrm{C} 2-\mathrm{C} 3$

$\mathrm{Na} 1{ }^{\mathrm{i}}-\mathrm{N} 2-\mathrm{C} 2-\mathrm{C} 3$

$\mathrm{N} 2-\mathrm{C} 2-\mathrm{C} 3-\mathrm{C} 4$

$\mathrm{C} 1-\mathrm{N} 3-\mathrm{C} 4-\mathrm{C} 3$

$\mathrm{Na} 1^{\mathrm{ii}}-\mathrm{N} 3-\mathrm{C} 4-\mathrm{C} 3$

$\mathrm{C} 2-\mathrm{C} 3-\mathrm{C} 4-\mathrm{N} 3$

$\mathrm{O} 2-\mathrm{S} 1-\mathrm{C} 5-\mathrm{C} 10$

$\mathrm{O} 1-\mathrm{S} 1-\mathrm{C} 5-\mathrm{C} 10$

$\mathrm{N} 1-\mathrm{S} 1-\mathrm{C} 5-\mathrm{C} 10$

$\mathrm{O} 2-\mathrm{S} 1-\mathrm{C} 5-\mathrm{C} 6$
$123.76(17)$

$123.45(15)$

$112.79(15)$

$168.70(13)$

$57.80(9)$

$56.52(8)$

123.65 (17)

118.2

118.2

115.45 (19)

122.3

122.3

$123.66(18)$

118.2

118.2

119.51 (17)

$121.98(14)$

118.51 (13)

121.10 (16)

119.5

119.5

119.79 (17)

120.1

120.1

119.84 (17)

$121.08(16)$

119.01 (17)

121.26 (16)

119.4

119.4

119.32 (17)

120.3

120.3

$13.88(16)$

162.33 (16)

$-1.3(3)$

139.15 (18)

$-3.2(3)$

0.3 (3)

-164.85 (18)

3.8 (3)

141.97 (15)

20.87 (18)

-103.42 (16)

-37.32 (16) 


\begin{tabular}{|c|c|}
\hline $\mathrm{O} 1-\mathrm{S} 1-\mathrm{C} 5-\mathrm{C} 6$ & $-158.41(14)$ \\
\hline $\mathrm{N} 1-\mathrm{S} 1-\mathrm{C} 5-\mathrm{C} 6$ & $77.29(16)$ \\
\hline $\mathrm{C} 10-\mathrm{C} 5-\mathrm{C} 6-\mathrm{C} 7$ & $0.3(3)$ \\
\hline $\mathrm{S} 1-\mathrm{C} 5-\mathrm{C} 6-\mathrm{C} 7$ & $179.62(15)$ \\
\hline $\mathrm{C} 5-\mathrm{C} 6-\mathrm{C} 7-\mathrm{C} 8$ & $-0.5(3)$ \\
\hline $\mathrm{Na}{ }^{\mathrm{iv}}-\mathrm{N} 4-\mathrm{C} 8-\mathrm{C} 7$ & $81.0(2)$ \\
\hline $\mathrm{Na}{ }^{\mathrm{iv}}-\mathrm{N} 4-\mathrm{C} 8-\mathrm{C} 9$ & $-95.8(2)$ \\
\hline $\mathrm{C} 6-\mathrm{C} 7-\mathrm{C} 8-\mathrm{N} 4$ & $-176.79(18)$ \\
\hline $\mathrm{C} 6-\mathrm{C} 7-\mathrm{C} 8-\mathrm{C} 9$ & $0.1(3)$ \\
\hline $\mathrm{N} 4-\mathrm{C} 8-\mathrm{C} 9-\mathrm{C} 10$ & $177.32(18)$ \\
\hline $\mathrm{C} 7-\mathrm{C} 8-\mathrm{C} 9-\mathrm{C} 10$ & $0.4(3)$ \\
\hline $\mathrm{C} 8-\mathrm{C} 9-\mathrm{C} 10-\mathrm{C} 5$ & $-0.6(3)$ \\
\hline $\mathrm{C} 6-\mathrm{C} 5-\mathrm{C} 10-\mathrm{C} 9$ & $0.2(3)$ \\
\hline $\mathrm{S} 1-\mathrm{C} 5-\mathrm{C} 10-\mathrm{C} 9$ & $-179.03(15)$ \\
\hline
\end{tabular}

Symmetry codes: (i) $-x+1,-y,-z$; (ii) $-x,-y,-z$; (iii) $-x+1, y-1 / 2,-z+1 / 2$; (iv) $-x+1, y+1 / 2,-z+1 / 2$.

Hydrogen-bond geometry $\left(\AA,{ }^{\circ}\right)$

\begin{tabular}{lllll}
\hline$D-\mathrm{H} \cdots A$ & $D-\mathrm{H}$ & $\mathrm{H} \cdots A$ & $D \cdots A$ & $D-\mathrm{H} \cdots A$ \\
\hline $\mathrm{N} 4-\mathrm{H} 1 N \cdots \mathrm{O} 2^{\mathrm{v}}$ & $0.86(2)$ & $2.06(2)$ & $2.907(2)$ & $166(2)$ \\
$\mathrm{N} 4-\mathrm{H} 2 N \cdots \mathrm{O} 1^{\text {iv }}$ & $0.91(2)$ & $2.52(2)$ & $2.959(2)$ & $110.5(17)$
\end{tabular}

Symmetry codes: (iv) $-x+1, y+1 / 2,-z+1 / 2$; (v) $-x, y+1 / 2,-z+1 / 2$.

(III)

\section{Crystal data}

$\mathrm{C}_{10} \mathrm{H}_{13} \mathrm{KN}_{4} \mathrm{O}_{4} \mathrm{~S}$

$M_{r}=324.40$

Triclinic, $P \overline{1}$

$a=8.8503(7) \AA$

$b=9.6385(4) \AA$

$c=15.9734(7) \AA$

$\alpha=92.350(4)^{\circ}$

$\beta=95.186(4)^{\circ}$

$\gamma=94.209(4)^{\circ}$

$V=1351.79(13) \AA^{3}$

Data collection

Oxford Diffraction Gemini S diffractometer

Radiation source: sealed tube $\omega$ scans

Absorption correction: multi-scan

CrysAlis PRO, Agilent Technologies, Version

1.171.37.35 (release 13-08-2014 CrysAlis 171

.NET) (compiled Aug 13 2014,18:06:01)

Empirical absorption correction using spherical harmonics, implemented in SCALE3

ABSPACK scaling algorithm.

$T_{\min }=0.601, T_{\max }=1.000$

15580 measured reflections

5357 independent reflections
$Z=4$

$F(000)=672$

$D_{\mathrm{x}}=1.594 \mathrm{Mg} \mathrm{m}^{-3}$

$\mathrm{Cu} K \alpha$ radiation, $\lambda=1.5418 \AA$

Cell parameters from 3605 reflections

$\theta=4.6-73.6^{\circ}$

$\mu=5.09 \mathrm{~mm}^{-1}$

$T=123 \mathrm{~K}$

Block, colourless

$0.28 \times 0.15 \times 0.10 \mathrm{~mm}$

3951 reflections with $I>2 \sigma(I)$

$R_{\text {int }}=0.050$

$\theta_{\text {max }}=73.8^{\circ}, \theta_{\min }=4.6^{\circ}$

$h=-8 \rightarrow 10$

$k=-11 \rightarrow 11$

$l=-19 \rightarrow 19$ 


\section{Refinement}

Refinement on $F^{2}$

Least-squares matrix: full

$R\left[F^{2}>2 \sigma\left(F^{2}\right)\right]=0.060$

$w R\left(F^{2}\right)=0.157$

$S=1.05$

5357 reflections

404 parameters

14 restraints

\section{Special details}

Geometry. All e.s.d.'s (except the e.s.d. in the dihedral angle between two 1.s. planes) are estimated using the full covariance matrix. The cell e.s.d.'s are taken into account individually in the estimation of e.s.d.'s in distances, angles and torsion angles; correlations between e.s.d.'s in cell parameters are only used when they are defined by crystal symmetry. An approximate (isotropic) treatment of cell e.s.d.'s is used for estimating e.s.d.'s involving l.s. planes.

Fractional atomic coordinates and isotropic or equivalent isotropic displacement parameters $\left(\AA^{2}\right)$

\begin{tabular}{|c|c|c|c|c|c|}
\hline & $x$ & $y$ & $z$ & $U_{\text {iso }} * / U_{\text {eq }}$ & Occ. $(<1)$ \\
\hline K1 & $0.24189(11)$ & $0.55756(9)$ & $0.47536(6)$ & $0.0350(2)$ & \\
\hline $\mathrm{K} 2$ & $-0.15927(12)$ & $0.82674(9)$ & $0.41066(6)$ & $0.0365(2)$ & \\
\hline $\mathrm{S} 1$ & $-0.26644(12)$ & $0.67031(10)$ & $0.19910(6)$ & $0.0304(2)$ & \\
\hline $\mathrm{S} 2$ & $-0.05315(12)$ & $0.81132(10)$ & $0.61877(6)$ & $0.0281(2)$ & \\
\hline O1 & $-0.3523(3)$ & $0.6886(3)$ & $0.27240(19)$ & $0.0345(7)$ & \\
\hline $\mathrm{O} 2$ & $-0.3075(4)$ & $0.7607(3)$ & $0.1321(2)$ & $0.0398(7)$ & \\
\hline $\mathrm{O} 1 \mathrm{~W}$ & $0.4746(5)$ & $0.4378(5)$ & $0.5915(3)$ & 0.0735 (14) & \\
\hline $\mathrm{H} 1 \mathrm{~W}$ & $0.450(9)$ & $0.398(6)$ & $0.637(2)$ & $0.110^{*}$ & \\
\hline $\mathrm{H} 2 \mathrm{~W}$ & $0.481(10)$ & $0.362(4)$ & $0.560(4)$ & $0.110^{*}$ & \\
\hline $\mathrm{O} 3$ & $-0.0548(3)$ & $0.9494(3)$ & $0.58530(18)$ & $0.0326(7)$ & \\
\hline $\mathrm{O} 2 \mathrm{~W}$ & $0.2375(5)$ & $0.8119(4)$ & $0.3937(2)$ & $0.0549(10)$ & \\
\hline $\mathrm{H} 3 \mathrm{~W}$ & $0.196(7)$ & $0.891(4)$ & $0.398(4)$ & $0.082^{*}$ & \\
\hline H4W & $0.199(7)$ & $0.785(6)$ & $0.3424(17)$ & $0.082 *$ & \\
\hline $\mathrm{O} 4$ & $-0.1743(4)$ & $0.7140(3)$ & $0.57871(18)$ & $0.0346(7)$ & \\
\hline $\mathrm{O} 3 \mathrm{~W}$ & -0.0485 (4) & $0.5553(3)$ & 0.39403 (19) & $0.0359(7)$ & \\
\hline H5W & $-0.050(6)$ & $0.584(4)$ & $0.3428(13)$ & $0.054 *$ & \\
\hline H6W & $-0.068(6)$ & $0.4658(13)$ & $0.384(3)$ & $0.054^{*}$ & \\
\hline O4W & $-0.4475(5)$ & $0.8724(4)$ & $0.4641(3)$ & $0.0604(11)$ & \\
\hline $\mathrm{H} 7 \mathrm{~W}$ & $-0.541(3)$ & $0.843(7)$ & $0.445(4)$ & $0.091 *$ & \\
\hline H8W & $-0.466(9)$ & $0.954(7)$ & $0.486(9)$ & $0.091 *$ & 0.5 \\
\hline H9W & $-0.436(10)$ & $0.819(11)$ & $0.507(5)$ & $0.091 *$ & 0.5 \\
\hline N1 & $-0.0933(4)$ & 0.6879 & $0.2369(2)$ & $0.0301(8)$ & \\
\hline N2 & $0.1612(4)$ & $0.7098(4)$ & $0.2256(2)$ & $0.0342(8)$ & \\
\hline N3 & $-0.0068(4)$ & $0.6541(4)$ & $0.1017(2)$ & $0.0348(8)$ & \\
\hline N4 & $-0.4201(6)$ & $0.0807(5)$ & 0.0779 & $0.0504(12)$ & \\
\hline N5 & $0.0982(4)$ & $0.7389(4)$ & $0.6097(2)$ & $0.0317(8)$ & \\
\hline N6 & $0.2546(4)$ & $0.9310(3)$ & $0.6755(2)$ & $0.0303(8)$ & \\
\hline N7 & $0.3546(5)$ & $0.7193(4)$ & $0.6308(2)$ & $0.0384(9)$ & \\
\hline N8 & $-0.1718(6)$ & $0.8726(5)$ & $0.9774(3)$ & $0.0441(10)$ & \\
\hline $\mathrm{C} 1$ & $0.0218(5)$ & $0.6828(4)$ & $0.1849(3)$ & $0.0309(9)$ & \\
\hline
\end{tabular}

Hydrogen site location: mixed

$\mathrm{H}$ atoms treated by a mixture of independent

and constrained refinement

$w=1 /\left[\sigma^{2}\left(F_{\mathrm{o}}^{2}\right)+(0.0619 P)^{2}+2.5308 P\right]$

where $P=\left(F_{\mathrm{o}}^{2}+2 F_{\mathrm{c}}{ }^{2}\right) / 3$

$(\Delta / \sigma)_{\max }<0.001$
$\Delta \rho_{\max }=0.81 \mathrm{e} \AA^{-3}$

$\Delta \rho_{\min }=-0.45$ e $\AA^{-3}$ 


$\begin{array}{lllll}\text { C2 } & 0.2782(6) & 0.7163(5) & 0.1785(3) & 0.0433(11) \\ \text { H2 } & 0.3781 & 0.7352 & 0.2055 & 0.052^{*} \\ \text { C3 } & 0.2599(6) & 0.6967(5) & 0.0925(3) & 0.0447(12) \\ \text { H3 } & 0.3436 & 0.7068 & 0.0593 & 0.054^{*} \\ \text { C4 } & 0.1144(6) & 0.6617(5) & 0.0567(3) & 0.0406(11) \\ \text { H4 } & 0.0987 & 0.6421 & -0.0023 & 0.049^{*} \\ \text { C5 } & -0.3070(5) & 0.4959(4) & 0.1601(3) & 0.0299(9) \\ \text { C6 } & -0.2140(5) & 0.3935(4) & 0.1847(3) & 0.0312(9) \\ \text { H6 } & -0.1236 & 0.4172 & 0.2207 & 0.037^{*} \\ \text { C7 } & -0.2516(5) & 0.2566(5) & 0.1571(3) & 0.0353(10) \\ \text { H7 } & -0.1851 & 0.1873 & 0.1732 & 0.042^{*} \\ \text { C8 } & -0.3841(5) & 0.2186(5) & 0.1065(3) & 0.0377(11) \\ \text { C9 } & -0.4762(6) & 0.3219(6) & 0.0812(3) & 0.0480(13) \\ \text { H9 } & -0.5667 & 0.2978 & 0.0453 & 0.058^{*} \\ \text { C10 } & -0.4386(5) & 0.4598(5) & 0.1073(3) & 0.0436(12) \\ \text { H10 } & -0.5028 & 0.5297 & 0.0892 & 0.052^{*} \\ \text { C11 } & 0.2388(5) & 0.8013(4) & 0.6399(3) & 0.0295(9) \\ \text { C12 } & 0.3946(5) & 0.9760(4) & 0.7084(3) & 0.0339(10) \\ \text { H12 } & 0.4095 & 1.0670 & 0.7344 & 0.041^{*} \\ \text { C13 } & 0.5188(5) & 0.8976(5) & 0.7068(3) & 0.0384(10) \\ \text { H13 } & 0.6166 & 0.9299 & 0.7326 & 0.046^{*} \\ \text { C14 } & 0.4908(6) & 0.7693(5) & 0.6650(3) & 0.0428(11) \\ \text { H14 } & 0.5740 & 0.7133 & 0.6603 & 0.051^{*} \\ \text { C15 } & -0.0866(5) & 0.8295(4) & 0.7260(2) & 0.0269(8) \\ \text { C16 } & -0.1369(5) & 0.7129(4) & 0.7677(3) & 0.0298(9) \\ \text { H16 } & -0.1542 & 0.6246 & 0.7382 & 0.036^{*} \\ \text { C17 } & -0.1615(5) & 0.7256(4) & 0.8511(3) & 0.0334(10) \\ \text { H17 } & -0.1935 & 0.6454 & 0.8794 & 0.040^{*} \\ \text { C18 } & -0.1399(5) & 0.8561(4) & 0.8955(3) & 0.0313(9) \\ \text { C19 } & -0.0884(5) & 0.9708(4) & 0.8523(3) & 0.0300(9) \\ \text { H19 } & -0.0723 & 1.0597 & 0.8810 & 0.036^{*} \\ \text { C20 } & -0.0605(5) & 0.9583(4) & 0.7689(3) & 0.0281(9) \\ \text { H20 } & -0.0236 & 1.0373 & 0.7410 & 0.034^{*} \\ \text { H1N } & -0.515(8) & 0.057(7) & 0.059(4) & 0.07(2)^{*} \\ \text { H2N } & -0.383(7) & 0.010(7) & 0.111(4) & 0.06(2)^{*} \\ \text { H3N } & -0.185(6) & 0.800(5) & 1.008(3) & 0.037(14)^{*} \\ \text { H4N } & -0.156(6) & 0.955(6) & 1.004(3) & 0.053(16)^{*} \\ & & & & \end{array}$

Atomic displacement parameters $\left(\AA^{2}\right)$

\begin{tabular}{lllllll}
\hline & $U^{11}$ & $U^{22}$ & $U^{33}$ & $U^{12}$ & $U^{13}$ & $U^{23}$ \\
\hline $\mathrm{K} 1$ & $0.0413(5)$ & $0.0269(5)$ & $0.0355(5)$ & $-0.0036(4)$ & $0.0044(4)$ & $-0.0060(4)$ \\
$\mathrm{K} 2$ & $0.0504(6)$ & $0.0234(4)$ & $0.0337(5)$ & $-0.0016(4)$ & $-0.0020(4)$ & $-0.0007(3)$ \\
$\mathrm{S} 1$ & $0.0337(6)$ & $0.0274(5)$ & $0.0301(5)$ & $0.0045(4)$ & $0.0037(4)$ & $-0.0023(4)$ \\
$\mathrm{S} 2$ & $0.0327(5)$ & $0.0236(5)$ & $0.0269(5)$ & $-0.0012(4)$ & $0.0021(4)$ & $-0.0022(4)$ \\
$\mathrm{O} 1$ & $0.0344(17)$ & $0.0338(16)$ & $0.0354(16)$ & $0.0017(13)$ & $0.0071(13)$ & $-0.0064(13)$ \\
$\mathrm{O} 2$ & $0.046(2)$ & $0.0372(17)$ & $0.0375(17)$ & $0.0119(14)$ & $0.0050(14)$ & $0.0017(13)$ \\
O1W & $0.048(2)$ & $0.079(3)$ & $0.100(4)$ & $0.009(2)$ & $0.018(2)$ & $0.049(3)$
\end{tabular}




\begin{tabular}{|c|c|c|c|c|c|c|c|}
\hline 457 & $\mathrm{O} 3$ & $0.0342(17)$ & $0.0296(15)$ & $0.0339(16)$ & $0.0023(13)$ & $0.0021(12)$ & $0.0019(12)$ \\
\hline 458 & $\mathrm{O} 2 \mathrm{~W}$ & $0.077(3)$ & $0.0357(19)$ & $0.050(2)$ & $0.0102(19)$ & $-0.0048(19)$ & $-0.0027(16)$ \\
\hline 459 & $\mathrm{O} 4$ & $0.0381(17)$ & $0.0325(16)$ & $0.0310(15)$ & $-0.0049(13)$ & $0.0005(13)$ & $-0.0043(12)$ \\
\hline 460 & $\mathrm{O} 3 \mathrm{~W}$ & 0.0464 (19) & $0.0274(15)$ & $0.0330(16)$ & 0.0014 (14) & $0.0028(14)$ & $-0.0039(12)$ \\
\hline 461 & O4W & $0.079(3)$ & $0.039(2)$ & $0.067(3)$ & 0.0007 (19) & $0.031(2)$ & $0.0003(18)$ \\
\hline 462 & N1 & $0.036(2)$ & $0.0247(17)$ & $0.0286(18)$ & $-0.0033(15)$ & $0.0060(15)$ & $-0.0011(14)$ \\
\hline 463 & $\mathrm{~N} 2$ & $0.033(2)$ & $0.0271(18)$ & $0.043(2)$ & $0.0030(15)$ & $0.0090(16)$ & $0.0011(15)$ \\
\hline 464 & N3 & 0.045 (2) & $0.0241(18)$ & $0.036(2)$ & $0.0029(16)$ & $0.0113(17)$ & $-0.0007(15)$ \\
\hline 465 & N4 & 0.057 (3) & $0.045(3)$ & 0.045 & $-0.017(2)$ & $0.010(2)$ & $-0.020(2)$ \\
\hline 466 & N5 & 0.040 (2) & $0.0240(17)$ & 0.0315 (19) & $0.0023(15)$ & $0.0070(15)$ & $-0.0046(14)$ \\
\hline 467 & N6 & $0.033(2)$ & $0.0240(17)$ & 0.0340 (19) & $-0.0005(14)$ & $0.0040(15)$ & $-0.0004(14)$ \\
\hline 468 & N7 & $0.045(2)$ & $0.034(2)$ & $0.037(2)$ & $0.0081(17)$ & 0.0049 (17) & $-0.0048(16)$ \\
\hline 469 & $\mathrm{~N} 8$ & $0.071(3)$ & $0.031(2)$ & $0.031(2)$ & $-0.002(2)$ & $0.0101(19)$ & $-0.0008(17)$ \\
\hline 470 & $\mathrm{C} 1$ & 0.038 (2) & $0.0164(19)$ & 0.039 (2) & $0.0026(17)$ & $0.0089(19)$ & $0.0003(16)$ \\
\hline 471 & $\mathrm{C} 2$ & $0.035(3)$ & $0.033(2)$ & $0.064(3)$ & $0.007(2)$ & $0.012(2)$ & $0.010(2)$ \\
\hline 472 & $\mathrm{C} 3$ & $0.043(3)$ & $0.041(3)$ & $0.057(3)$ & $0.015(2)$ & $0.023(2)$ & 0.011 (2) \\
\hline 473 & $\mathrm{C} 4$ & 0.059 (3) & $0.029(2)$ & $0.038(2)$ & 0.009 (2) & $0.017(2)$ & $0.0040(18)$ \\
\hline 474 & $\mathrm{C} 5$ & $0.028(2)$ & $0.032(2)$ & $0.029(2)$ & $0.0021(17)$ & $0.0035(16)$ & $-0.0066(17)$ \\
\hline 475 & C6 & $0.032(2)$ & $0.029(2)$ & $0.031(2)$ & $0.0000(18)$ & $-0.0012(17)$ & $-0.0006(17)$ \\
\hline 476 & $\mathrm{C} 7$ & $0.043(3)$ & $0.030(2)$ & $0.033(2)$ & 0.0007 (19) & $0.0027(19)$ & $-0.0010(17)$ \\
\hline 477 & $\mathrm{C} 8$ & $0.037(3)$ & $0.040(2)$ & $0.035(2)$ & $-0.008(2)$ & 0.0099 (19) & $-0.0118(19)$ \\
\hline 478 & C9 & $0.031(3)$ & 0.060 & $0.048(3)$ & $-0.004(2)$ & $-0.002(2)$ & $-0.028(2)$ \\
\hline 479 & $\mathrm{C} 10$ & $0.030(2)$ & $0.054(3)$ & $0.046(3)$ & $0.016(2)$ & $-0.004(2)$ & $-0.017(2)$ \\
\hline 480 & C11 & $0.035(2)$ & $0.027(2)$ & $0.028(2)$ & $0.0019(17)$ & $0.0058(17)$ & $0.0002(16)$ \\
\hline 481 & $\mathrm{C} 12$ & $0.036(2)$ & $0.028(2)$ & $0.037(2)$ & $-0.0023(18)$ & 0.0018 (19) & $-0.0017(17)$ \\
\hline 482 & $\mathrm{C} 13$ & $0.023(2)$ & $0.047(3)$ & $0.044(3)$ & 0.0054 (19) & $-0.0007(18)$ & $-0.001(2)$ \\
\hline 483 & $\mathrm{C} 14$ & $0.036(3)$ & $0.052(3)$ & $0.042(3)$ & $0.018(2)$ & $0.004(2)$ & $0.000(2)$ \\
\hline 484 & $\mathrm{C} 15$ & $0.028(2)$ & $0.026(2)$ & $0.027(2)$ & $0.0014(16)$ & $0.0041(16)$ & $-0.0019(15)$ \\
\hline 485 & $\mathrm{C} 16$ & $0.030(2)$ & $0.022(2)$ & $0.036(2)$ & $0.0004(16)$ & $0.0022(17)$ & $-0.0044(16)$ \\
\hline 486 & $\mathrm{C} 17$ & $0.037(2)$ & $0.022(2)$ & $0.041(2)$ & $-0.0012(18)$ & 0.0028 (19) & 0.0044 (17) \\
\hline 487 & $\mathrm{C} 18$ & $0.030(2)$ & $0.031(2)$ & $0.032(2)$ & $0.0013(18)$ & $0.0016(17)$ & $-0.0023(17)$ \\
\hline 488 & C19 & $0.029(2)$ & $0.023(2)$ & $0.036(2)$ & $0.0024(17)$ & $0.0001(17)$ & $-0.0061(16)$ \\
\hline 489 & $\mathrm{C} 20$ & $0.025(2)$ & $0.0224(19)$ & $0.036(2)$ & $0.0003(16)$ & $0.0016(16)$ & $0.0004(16)$ \\
\hline
\end{tabular}

Geometric parameters $\left(A,{ }^{\circ}\right)$

\begin{tabular}{llll}
\hline $\mathrm{K} 1-\mathrm{O} 4^{\mathrm{i}}$ & $2.729(3)$ & $\mathrm{N} 1-\mathrm{C} 1$ & $1.373(5)$ \\
$\mathrm{K} 1-\mathrm{O} 3 \mathrm{~W}$ & $2.771(3)$ & $\mathrm{N} 2-\mathrm{C} 2$ & $1.333(6)$ \\
$\mathrm{K} 1-\mathrm{O} 1 W^{\mathrm{ii}}$ & $2.812(5)$ & $\mathrm{N} 2-\mathrm{C} 1$ & $1.343(6)$ \\
$\mathrm{K} 1-\mathrm{O} 2 \mathrm{~W}$ & $2.824(4)$ & $\mathrm{N} 3-\mathrm{C} 4$ & $1.343(6)$ \\
$\mathrm{K} 1-\mathrm{N} 7$ & $2.932(4)$ & $\mathrm{N} 3-\mathrm{C} 1$ & $1.344(6)$ \\
$\mathrm{K} 1-\mathrm{O} 1 \mathrm{~W}$ & $2.975(5)$ & $\mathrm{N} 4-\mathrm{C} 8$ & $1.393(6)$ \\
$\mathrm{K} 1-\mathrm{O} 3 W^{\mathrm{i}}$ & $3.004(3)$ & $\mathrm{N} 4-\mathrm{H} 1 \mathrm{~N}$ & $0.87(7)$ \\
$\mathrm{K} 1-\mathrm{N} 5$ & $3.129(4)$ & $\mathrm{N} 4-\mathrm{H} 2 \mathrm{~N}$ & $0.93(7)$ \\
$\mathrm{K} 1-\mathrm{C} 11$ & $3.457(4)$ & $\mathrm{N} 5-\mathrm{C} 11$ & $1.379(5)$ \\
$\mathrm{K} 1-\mathrm{K} 2^{\mathrm{i}}$ & $4.2385(13)$ & $\mathrm{N} 6-\mathrm{C} 12$ & $1.336(6)$ \\
$\mathrm{K} 1-\mathrm{K} 1^{\mathrm{i}}$ & $4.491(2)$ & $\mathrm{N} 6-\mathrm{C} 11$ & $1.344(5)$ \\
$\mathrm{K} 1-\mathrm{K} 2$ & $4.6167(15)$ & $\mathrm{N} 6-\mathrm{K} 2^{\mathrm{iii}}$ & $2.899(4)$
\end{tabular}


K1-H6W

$\mathrm{K} 2-\mathrm{O} 3^{\mathrm{iii}}$

$\mathrm{K} 2-\mathrm{O} 4 \mathrm{~W}$

$\mathrm{K} 2-\mathrm{O} 3 \mathrm{~W}$

$\mathrm{K} 2-\mathrm{O} 1$

$\mathrm{K} 2-\mathrm{N} 6^{\mathrm{iii}}$

$\mathrm{K} 2-\mathrm{O} 4$

$\mathrm{K} 2-\mathrm{O} 3$

$\mathrm{K} 2-\mathrm{N} 1$

$\mathrm{K} 2-\mathrm{S} 2$

$\mathrm{K} 2-\mathrm{C} 12^{\mathrm{iii}}$

$\mathrm{K} 2-\mathrm{S} 1$

$\mathrm{K} 2-\mathrm{K} 1^{\mathrm{i}}$

$\mathrm{K} 2-\mathrm{H} 5 \mathrm{~W}$

K2-H9W

$\mathrm{S} 1-\mathrm{O} 2$

$\mathrm{S} 1-\mathrm{O} 1$

$\mathrm{S} 1-\mathrm{N} 1$

$\mathrm{S} 1-\mathrm{C} 5$

$\mathrm{S} 2-\mathrm{O} 3$

$\mathrm{S} 2-\mathrm{O} 4$

$\mathrm{S} 2-\mathrm{N} 5$

$\mathrm{S} 2-\mathrm{C} 15$

$\mathrm{O} 1 \mathrm{~W}-\mathrm{K} 1^{\mathrm{ii}}$

O1W-H1W

$\mathrm{O} 1 \mathrm{~W}-\mathrm{H} 2 \mathrm{~W}$

$\mathrm{O} 3-\mathrm{K} 2^{\mathrm{iii}}$

$\mathrm{O} 2 \mathrm{~W}-\mathrm{H} 3 \mathrm{~W}$

$\mathrm{O} 2 \mathrm{~W}-\mathrm{H} 4 \mathrm{~W}$

$\mathrm{O} 4-\mathrm{K} 1^{\mathrm{i}}$

$\mathrm{O} 3 \mathrm{~W}-\mathrm{K} 1^{\mathrm{i}}$

$\mathrm{O} 3 \mathrm{~W}-\mathrm{H} 5 \mathrm{~W}$

O3W-H6W

$\mathrm{O} 4 \mathrm{~W}-\mathrm{H} 7 \mathrm{~W}$

$\mathrm{O} 4 \mathrm{~W}-\mathrm{H} 8 \mathrm{~W}$

O4W-H9W

$\mathrm{O} 4-\mathrm{K} 1-\mathrm{O} 3 \mathrm{~W}$

$\mathrm{O} 4-\mathrm{K} 1-\mathrm{O} 1 \mathrm{~W}^{\mathrm{ii}}$

$\mathrm{O} 3 \mathrm{~W}-\mathrm{K} 1-\mathrm{O} 1 \mathrm{~W}^{\mathrm{ii}}$

$\mathrm{O} 4-\mathrm{K} 1-\mathrm{O} 2 \mathrm{~W}$

$\mathrm{O} 3 \mathrm{~W}-\mathrm{K} 1-\mathrm{O} 2 \mathrm{~W}$

$\mathrm{O} 1 \mathrm{~W}$ ii-K1-O2W

O4 - K1-N7

$\mathrm{O} 3 \mathrm{~W}-\mathrm{K} 1-\mathrm{N} 7$

$\mathrm{O} 1 \mathrm{~W}$ ii $-\mathrm{K} 1-\mathrm{N} 7$

$\mathrm{O} 2 \mathrm{~W}-\mathrm{K} 1-\mathrm{N} 7$

$\mathrm{O} 4-\mathrm{K} 1-\mathrm{O} 1 \mathrm{~W}$
$3.04(5)$

$2.759(3)$

$2.820(4)$

$2.871(3)$

$2.883(3)$

$2.899(4)$

2.947 (3)

3.023 (3)

$3.153(3)$

3.3840 (14)

$3.425(5)$

3.6587 (14)

$4.2385(13)$

$2.81(5)$

$3.01(11)$

1.445 (3)

$1.464(3)$

$1.589(4)$

$1.769(4)$

$1.455(3)$

$1.455(3)$

$1.570(4)$

$1.768(4)$

$2.812(5)$

$0.880(10)$

$0.877(10)$

$2.759(3)$

$0.878(10)$

$0.880(10)$

2.729 (3)

3.004 (3)

0.874 (10)

$0.873(10)$

0.878 (10)

0.879 (10)

0.878 (10)

76.14 (9)

91.40 (13)

129.93 (13)

$133.37(10)$

75.00 (11)

80.19 (13)

138.88 (11)

125.77 (11)

$95.36(15)$

87.72 (11)

83.27 (12)
N7- 114

N7-C11

N8-C18

N8- $\mathrm{H} 3 \mathrm{~N}$

$\mathrm{N} 8-\mathrm{H} 4 \mathrm{~N}$

$\mathrm{C} 2-\mathrm{C} 3$

$\mathrm{C} 2-\mathrm{H} 2$

$\mathrm{C} 3-\mathrm{C} 4$

C3- 33

C4- $-\mathrm{H} 4$

C5-C6

C5- $\mathrm{C} 10$

C6- 77

C6- $-\mathrm{H} 6$

C7-C8

C7- 77

C8-C9

C9- $\mathrm{C} 10$

C9- $\mathrm{H} 9$

$\mathrm{C} 10-\mathrm{H} 10$

C12-C13

$\mathrm{C} 12-\mathrm{K} 2^{\mathrm{iii}}$

C12-H12

C13-C14

C13-H13

C14-H14

C15-C20

C15-C16

C16-C17

C16-H16

C17-C18

C17-H17

C18-C19

C19-C20

C19-H19

$\mathrm{C} 20-\mathrm{H} 20$

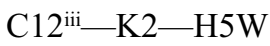

$\mathrm{S} 1-\mathrm{K} 2-\mathrm{H} 5 \mathrm{~W}$

$\mathrm{K} 1{ }^{\mathrm{i}}-\mathrm{K} 2-\mathrm{H} 5 \mathrm{~W}$

O3 3 iii-K2-H9W

$\mathrm{O} 4 \mathrm{~W}-\mathrm{K} 2-\mathrm{H} 9 \mathrm{~W}$

$\mathrm{O} 3 \mathrm{~W}-\mathrm{K} 2-\mathrm{H} 9 \mathrm{~W}$

$\mathrm{O} 1-\mathrm{K} 2-\mathrm{H} 9 \mathrm{~W}$

$\mathrm{N} 6{ }^{\mathrm{iii}}-\mathrm{K} 2-\mathrm{H} 9 \mathrm{~W}$

$\mathrm{O} 4-\mathrm{K} 2-\mathrm{H} 9 \mathrm{~W}$

O3-K2- $\mathrm{H} 9 \mathrm{~W}$

$\mathrm{N} 1-\mathrm{K} 2-\mathrm{H} 9 \mathrm{~W}$
$1.325(6)$

$1.355(6)$

$1.369(6)$

0.87 (5)

0.88 (6)

$1.372(7)$

0.9500

1.373 (7)

0.9500

0.9500

$1.378(6)$

$1.388(6)$

$1.382(6)$

0.9500

$1.379(6)$

0.9500

$1.383(7)$

$1.385(7)$

0.9500

0.9500

$1.380(6)$

$3.425(5)$

0.9500

$1.376(7)$

0.9500

0.9500

$1.387(5)$

$1.395(6)$

$1.372(6)$

0.9500

$1.410(6)$

0.9500

1.397 (6)

$1.378(6)$

0.9500

0.9500

$121.5(6)$

54.1 (7)

60.3 (5)

124 (2)

$17.0(8)$

111.2 (19)

87.0 (19)

91.0 (12)

55.4 (6)

73.4 (18)

133 (2) 
O3W-K1-O1W

$\mathrm{O} 1 \mathrm{~W}^{\mathrm{ii}}-\mathrm{K} 1-\mathrm{O} 1 \mathrm{~W}$

$\mathrm{O} 2 \mathrm{~W}-\mathrm{K} 1-\mathrm{O} 1 \mathrm{~W}$

N7-K1-O1W

$\mathrm{O} 4-\mathrm{K} 1-\mathrm{O} 3 \mathrm{~W}^{\mathrm{i}}$

$\mathrm{O} 3 \mathrm{~W}-\mathrm{K} 1-\mathrm{O}^{\mathrm{W}} \mathrm{W}^{\mathrm{i}}$

$\mathrm{O} 1 \mathrm{~W}^{\mathrm{ii}}-\mathrm{K} 1-\mathrm{O} 3 \mathrm{~W}^{\mathrm{i}}$

$\mathrm{O} 2 \mathrm{~W}-\mathrm{K} 1-\mathrm{O} 3 \mathrm{~W}^{\mathrm{i}}$

$\mathrm{N} 7-\mathrm{K} 1-\mathrm{O} 3 \mathrm{~W}^{\mathrm{i}}$

$\mathrm{O} 1 \mathrm{~W}-\mathrm{K} 1-\mathrm{O}_{3} \mathrm{~W}^{\mathrm{i}}$

$\mathrm{O} 4 \mathrm{i}-\mathrm{K} 1-\mathrm{N} 5$

$\mathrm{O} 3 \mathrm{~W}-\mathrm{K} 1-\mathrm{N} 5$

$\mathrm{O} 1 \mathrm{~W}^{\mathrm{ii}-\mathrm{K} 1-\mathrm{N} 5}$

$\mathrm{O} 2 \mathrm{~W}-\mathrm{K} 1-\mathrm{N} 5$

$\mathrm{N} 7-\mathrm{K} 1-\mathrm{N} 5$

$\mathrm{O} 1 \mathrm{~W}-\mathrm{K} 1-\mathrm{N} 5$

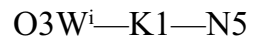

$\mathrm{O} 4 \mathrm{i}-\mathrm{K} 1-\mathrm{C} 11$

$\mathrm{O} 3 \mathrm{~W}-\mathrm{K} 1-\mathrm{C} 11$

$\mathrm{O} 1 \mathrm{~W}$ ii-K1-C11

$\mathrm{O} 2 \mathrm{~W}-\mathrm{K} 1-\mathrm{C} 11$

$\mathrm{N} 7-\mathrm{K} 1-\mathrm{C} 11$

$\mathrm{O} 1 \mathrm{~W}-\mathrm{K} 1-\mathrm{C} 11$

O3W $-\mathrm{K} 1-\mathrm{C} 11$

N5-K1-C11

$\mathrm{O} 4^{\mathrm{i}}-\mathrm{K} 1-\mathrm{K} 2^{\mathrm{i}}$

$\mathrm{O} 3 \mathrm{~W}-\mathrm{K} 1-\mathrm{K} 2^{\mathrm{i}}$

$\mathrm{O} 1 \mathrm{~W}^{\mathrm{ii}}-\mathrm{K} 1-\mathrm{K} 2^{\mathrm{i}}$

$\mathrm{O} 2 \mathrm{~W}-\mathrm{K} 1-\mathrm{K} 2^{\mathrm{i}}$

$\mathrm{N} 7-\mathrm{K} 1-\mathrm{K} 2^{\mathrm{i}}$

$\mathrm{O} 1 \mathrm{~W}-\mathrm{K} 1-\mathrm{K} 2^{\mathrm{i}}$

$\mathrm{O} 3 \mathrm{~W}^{\mathrm{i}}-\mathrm{K} 1-\mathrm{K} 2^{\mathrm{i}}$

$\mathrm{N} 5-\mathrm{K} 1-\mathrm{K} 2^{\mathrm{i}}$

$\mathrm{C} 11-\mathrm{K} 1-\mathrm{K} 2^{\mathrm{i}}$

$\mathrm{O} 4-\mathrm{K} 1-\mathrm{K} 1^{\mathrm{i}}$

$\mathrm{O} 3 \mathrm{~W}-\mathrm{K} 1-\mathrm{K} 1^{\mathrm{i}}$

$\mathrm{O} 1 \mathrm{~W}^{\mathrm{ii}}-\mathrm{K} 1-\mathrm{K} 1^{\mathrm{i}}$

$\mathrm{O} 2 \mathrm{~W}-\mathrm{K} 1-\mathrm{K} 1^{\mathrm{i}}$

$\mathrm{N} 7-\mathrm{K} 1-\mathrm{K} 1^{\mathrm{i}}$

$\mathrm{O} 1 \mathrm{~W}-\mathrm{K} 1-\mathrm{K} 1^{\mathrm{i}}$

$\mathrm{O} 3 \mathrm{~W}^{\mathrm{i}}-\mathrm{K} 1-\mathrm{K} 1^{\mathrm{i}}$

$\mathrm{N} 5-\mathrm{K} 1-\mathrm{K} 1^{\mathrm{i}}$

$\mathrm{C} 11-\mathrm{K} 1-\mathrm{K} 1^{\mathrm{i}}$

$\mathrm{K} 2^{\mathrm{i}}-\mathrm{K} 1-\mathrm{K} 1^{\mathrm{i}}$

$\mathrm{O} 4 \mathrm{i}-\mathrm{K} 1-\mathrm{K} 2$

$\mathrm{O} 3 \mathrm{~W}-\mathrm{K} 1-\mathrm{K} 2$

$\mathrm{O} 1 \mathrm{~W}^{\mathrm{ii}}-\mathrm{K} 1-\mathrm{K} 2$

$\mathrm{O} 2 \mathrm{~W}-\mathrm{K} 1-\mathrm{K} 2$
$152.02(11)$

68.49 (15)

$132.73(12)$

$62.11(14)$

76.90 (9)

$78.00(10)$

$146.61(12)$

$130.21(12)$

$75.36(10)$

79.04 (11)

$130.33(10)$

81.94 (10)

135.34 (13)

80.19 (11)

$44.19(10)$

$97.70(13)$

$55.02(9)$

$145.79(10)$

$103.38(10)$

$112.40(14)$

$76.90(10)$

$22.63(11)$

$83.25(13)$

$69.73(9)$

$23.50(10)$

$43.70(6)$

$94.71(7)$

$109.14(9)$

$169.33(10)$

$96.31(8)$

$57.39(8)$

$42.59(6)$

$95.81(7)$

$103.32(7)$

$72.58(7)$

40.87 (7)

$162.68(12)$

$105.79(10)$

$101.05(9)$

$114.70(9)$

$37.13(6)$

$61.97(7)$

$84.90(8)$

$63.78(3)$

$111.87(7)$

$35.80(6)$

128.87 (9)

$50.39(10)$
S2-K2-H9W

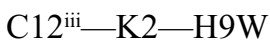

$\mathrm{S} 1-\mathrm{K} 2-\mathrm{H} 9 \mathrm{~W}$

$\mathrm{K} 1$ - $-\mathrm{K} 2-\mathrm{H} 9 \mathrm{~W}$

$\mathrm{H} 5 \mathrm{~W}-\mathrm{K} 2-\mathrm{H} 9 \mathrm{~W}$

$\mathrm{O} 2-\mathrm{S} 1-\mathrm{O} 1$

$\mathrm{O} 2-\mathrm{S} 1-\mathrm{N} 1$

$\mathrm{O} 1-\mathrm{S} 1-\mathrm{N} 1$

$\mathrm{O} 2-\mathrm{S} 1-\mathrm{C} 5$

$\mathrm{O} 1-\mathrm{S} 1-\mathrm{C} 5$

$\mathrm{N} 1-\mathrm{S} 1-\mathrm{C} 5$

$\mathrm{O} 2-\mathrm{S} 1-\mathrm{K} 2$

$\mathrm{O} 1-\mathrm{S} 1-\mathrm{K} 2$

$\mathrm{N} 1-\mathrm{S} 1-\mathrm{K} 2$

$\mathrm{C} 5-\mathrm{S} 1-\mathrm{K} 2$

$\mathrm{O} 3-\mathrm{S} 2-\mathrm{O} 4$

O3-S2-N5

$\mathrm{O} 4-\mathrm{S} 2-\mathrm{N} 5$

$\mathrm{O} 3-\mathrm{S} 2-\mathrm{C} 15$

$\mathrm{O} 4-\mathrm{S} 2-\mathrm{C} 15$

N5-S2-C15

$\mathrm{O} 3-\mathrm{S} 2-\mathrm{K} 2$

$\mathrm{O} 4-\mathrm{S} 2-\mathrm{K} 2$

N5-S2-K2

$\mathrm{C} 15-\mathrm{S} 2-\mathrm{K} 2$

$\mathrm{S} 1-\mathrm{O} 1-\mathrm{K} 2$

$\mathrm{K} 1$ ii- $\mathrm{O} 1 \mathrm{~W}-\mathrm{K} 1$

$\mathrm{K} 1 \mathrm{ii}-\mathrm{O} 1 \mathrm{~W}-\mathrm{H} 1 \mathrm{~W}$

$\mathrm{K} 1-\mathrm{O} 1 \mathrm{~W}-\mathrm{H} 1 \mathrm{~W}$

$\mathrm{K} 1 \mathrm{ii}-\mathrm{O} 1 \mathrm{~W}-\mathrm{H} 2 \mathrm{~W}$

$\mathrm{K} 1-\mathrm{O} 1 \mathrm{~W}-\mathrm{H} 2 \mathrm{~W}$

$\mathrm{H} 1 \mathrm{~W}-\mathrm{O} 1 \mathrm{~W}-\mathrm{H} 2 \mathrm{~W}$

$\mathrm{S} 2-\mathrm{O} 3-\mathrm{K} 22^{\mathrm{iii}}$

$\mathrm{S} 2-\mathrm{O} 3-\mathrm{K} 2$

$\mathrm{K} 2{ }^{\mathrm{iii}}-\mathrm{O} 3-\mathrm{K} 2$

$\mathrm{K} 1-\mathrm{O} 2 \mathrm{~W}-\mathrm{H} 3 \mathrm{~W}$

$\mathrm{K} 1-\mathrm{O} 2 \mathrm{~W}-\mathrm{H} 4 \mathrm{~W}$

$\mathrm{H} 3 \mathrm{~W}-\mathrm{O} 2 \mathrm{~W}-\mathrm{H} 4 \mathrm{~W}$

$\mathrm{S} 2-\mathrm{O} 4-\mathrm{K} 1^{\mathrm{i}}$

$\mathrm{S} 2-\mathrm{O} 4-\mathrm{K} 2$

$\mathrm{K} 1 \mathrm{i}-\mathrm{O} 4-\mathrm{K} 2$

$\mathrm{K} 1-\mathrm{O} 3 \mathrm{~W}-\mathrm{K} 2$

$\mathrm{K} 1-\mathrm{O} 3 \mathrm{~W}-\mathrm{K} 1^{\mathrm{i}}$

$\mathrm{K} 2-\mathrm{O} 3 \mathrm{~W}-\mathrm{K} 1^{\mathrm{i}}$

$\mathrm{K} 1-\mathrm{O} 3 \mathrm{~W}-\mathrm{H} 5 \mathrm{~W}$

$\mathrm{K} 2-\mathrm{O} 3 \mathrm{~W}-\mathrm{H} 5 \mathrm{~W}$

$\mathrm{K} 1 \mathrm{i}-\mathrm{O} 3 \mathrm{~W}-\mathrm{H} 5 \mathrm{~W}$

$\mathrm{K} 1-\mathrm{O} 3 \mathrm{~W}-\mathrm{H} 6 \mathrm{~W}$
$70.2(11)$

$78.2(7)$

109.0 (18)

$67.2(16)$

$122(2)$

$113.35(19)$

$115.66(19)$

104.21 (18)

108.1 (2)

$107.12(18)$

107.9 (2)

118.44 (13)

47.64 (12)

$59.11(13)$

$132.72(15)$

$112.95(18)$

114.45 (19)

$105.51(19)$

106.97 (18)

$106.49(18)$

$110.2(2)$

63.26 (12)

60.28 (12)

97.00 (14)

152.44 (15)

$110.33(15)$

111.51 (15)

$126(5)$

122 (6)

69 (6)

$96(6)$

98 (2)

133.47 (17)

91.28 (13)

114.35 (10)

139 (4)

102 (4)

99 (2)

143.3 (2)

94.33 (14)

96.54 (9)

109.81 (10)

$102.00(10)$

92.33 (9)

114 (4)

77 (3)

144 (4)

99 (4) 
N7-K1-K2

$\mathrm{O} 1 \mathrm{~W}-\mathrm{K} 1-\mathrm{K} 2$

$\mathrm{O} 3 \mathrm{~W}^{\mathrm{i}}-\mathrm{K} 1-\mathrm{K} 2$

$\mathrm{N} 5-\mathrm{K} 1-\mathrm{K} 2$

$\mathrm{C} 11-\mathrm{K} 1-\mathrm{K} 2$

$\mathrm{K} 2 \mathrm{i}-\mathrm{K} 1-\mathrm{K} 2$

$\mathrm{K} 1{ }^{\mathrm{i}}-\mathrm{K} 1-\mathrm{K} 2$

$\mathrm{O} 4-\mathrm{K} 1-\mathrm{H} 6 \mathrm{~W}$

$\mathrm{O} 3 \mathrm{~W}-\mathrm{K} 1-\mathrm{H} 6 \mathrm{~W}$

$\mathrm{O} 1 \mathrm{~W}^{\mathrm{ii}}-\mathrm{K} 1-\mathrm{H} 6 \mathrm{~W}$

$\mathrm{O} 2 \mathrm{~W}-\mathrm{K} 1-\mathrm{H} 6 \mathrm{~W}$

N7-K1-H6W

$\mathrm{O} 1 \mathrm{~W}-\mathrm{K} 1-\mathrm{H} 6 \mathrm{~W}$

$\mathrm{O} 3 \mathrm{~W}^{\mathrm{i}}-\mathrm{K} 1-\mathrm{H} 6 \mathrm{~W}$

N5-K1-H6W

$\mathrm{C} 11-\mathrm{K} 1-\mathrm{H} 6 \mathrm{~W}$

$\mathrm{K} 2 \mathrm{i}-\mathrm{K} 1-\mathrm{H} 6 \mathrm{~W}$

$\mathrm{K} 1{ }^{\mathrm{i}}-\mathrm{K} 1-\mathrm{H} 6 \mathrm{~W}$

$\mathrm{K} 2-\mathrm{K} 1-\mathrm{H} 6 \mathrm{~W}$

$\mathrm{O} 3{ }^{\mathrm{iii}}-\mathrm{K} 2-\mathrm{O} 4 \mathrm{~W}$

$\mathrm{O} 3{ }^{\mathrm{iii}}-\mathrm{K} 2-\mathrm{O} 3 \mathrm{~W}$

$\mathrm{O} 4 \mathrm{~W}-\mathrm{K} 2-\mathrm{O} 3 \mathrm{~W}$

$\mathrm{O} 3{ }^{\mathrm{iii}}-\mathrm{K} 2-\mathrm{O} 1$

$\mathrm{O} 4 \mathrm{~W}-\mathrm{K} 2-\mathrm{O} 1$

$\mathrm{O} 3 \mathrm{~W}-\mathrm{K} 2-\mathrm{O} 1$

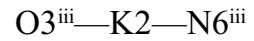

$\mathrm{O} 4 \mathrm{~W}-\mathrm{K} 2-\mathrm{N} 6^{\mathrm{iii}}$

$\mathrm{O} 3 \mathrm{~W}-\mathrm{K} 2-\mathrm{N}^{\mathrm{iii}}$

$\mathrm{O} 1-\mathrm{K} 2-\mathrm{N}^{6 i i}$

$\mathrm{O} 3{ }^{\mathrm{iii}}-\mathrm{K} 2-\mathrm{O} 4$

$\mathrm{O} 4 \mathrm{~W}-\mathrm{K} 2-\mathrm{O} 4$

$\mathrm{O} 3 \mathrm{~W}-\mathrm{K} 2-\mathrm{O} 4$

$\mathrm{O} 1-\mathrm{K} 2-\mathrm{O} 4$

$\mathrm{N} 6{ }^{\mathrm{iii}}-\mathrm{K} 2-\mathrm{O} 4$

$\mathrm{O} 3{ }^{\mathrm{iii}-\mathrm{K} 2-\mathrm{O} 3}$

$\mathrm{O} 4 \mathrm{~W}-\mathrm{K} 2-\mathrm{O} 3$

$\mathrm{O} 3 \mathrm{~W}-\mathrm{K} 2-\mathrm{O} 3$

$\mathrm{O} 1-\mathrm{K} 2-\mathrm{O} 3$

$\mathrm{N} 6{ }^{\mathrm{iii}}-\mathrm{K} 2-\mathrm{O} 3$

$\mathrm{O} 4-\mathrm{K} 2-\mathrm{O} 3$

$\mathrm{O} 3{ }^{\mathrm{iii}-\mathrm{K} 2}-\mathrm{N} 1$

$\mathrm{O} 4 \mathrm{~W}-\mathrm{K} 2-\mathrm{N} 1$

$\mathrm{O} 3 \mathrm{~W}-\mathrm{K} 2-\mathrm{N} 1$

$\mathrm{O} 1-\mathrm{K} 2-\mathrm{N} 1$

$\mathrm{N} 6{ }^{\mathrm{iii}-\mathrm{K} 2}-\mathrm{N} 1$

$\mathrm{O} 4-\mathrm{K} 2-\mathrm{N} 1$

$\mathrm{O} 3-\mathrm{K} 2-\mathrm{N} 1$

$\mathrm{O} 3{ }^{\mathrm{iii}-\mathrm{K} 2-\mathrm{S} 2}$
$94.90(8)$

$154.32(11)$

$84.29(7)$

$56.63(7)$

$72.66(8)$

119.23 (3)

$55.45(3)$

$59.8(5)$

16.5 (5)

$127.1(9)$

89.3 (5)

$136.2(8)$

$137.8(5)$

$73.6(8)$

$92.3(8)$

$115.3(8)$

80.9 (6)

38.7 (9)

$52.1(5)$

$118.31(10)$

$116.43(10)$

$123.26(11)$

130.90 (9)

80.08 (11)

75.53 (9)

$63.92(9)$

74.67 (11)

$146.26(10)$

80.79 (10)

$111.22(9)$

72.00 (12)

$75.72(9)$

117.84 (9)

$137.47(10)$

$65.65(10)$

$81.82(11)$

$108.46(9)$

$160.24(9)$

101.97 (9)

$47.93(8)$

97.57 (9)

$126.61(12)$

$56.72(9)$

$46.81(9)$

$89.55(10)$

131.79 (9)

$151.48(10)$

85.85 (7)
$\mathrm{K} 2-\mathrm{O} 3 \mathrm{~W}-\mathrm{H} 6 \mathrm{~W}$

$\mathrm{K} 1-\mathrm{O} 3 \mathrm{~W}-\mathrm{H} 6 \mathrm{~W}$

$\mathrm{H} 5 \mathrm{~W}-\mathrm{O} 3 \mathrm{~W}-\mathrm{H} 6 \mathrm{~W}$

$\mathrm{K} 2-\mathrm{O} 4 \mathrm{~W}-\mathrm{H} 7 \mathrm{~W}$

$\mathrm{K} 2-\mathrm{O} 4 \mathrm{~W}-\mathrm{H} 8 \mathrm{~W}$

$\mathrm{H} 7 \mathrm{~W}-\mathrm{O} 4 \mathrm{~W}-\mathrm{H} 8 \mathrm{~W}$

$\mathrm{K} 2-\mathrm{O} 4 \mathrm{~W}-\mathrm{H} 9 \mathrm{~W}$

$\mathrm{H} 7 \mathrm{~W}-\mathrm{O} 4 \mathrm{~W}-\mathrm{H} 9 \mathrm{~W}$

$\mathrm{C} 1-\mathrm{N} 1-\mathrm{S} 1$

$\mathrm{C} 1-\mathrm{N} 1-\mathrm{K} 2$

$\mathrm{S} 1-\mathrm{N} 1-\mathrm{K} 2$

$\mathrm{C} 2-\mathrm{N} 2-\mathrm{C} 1$

$\mathrm{C} 4-\mathrm{N} 3-\mathrm{C} 1$

$\mathrm{C} 8-\mathrm{N} 4-\mathrm{H} 1 \mathrm{~N}$

$\mathrm{C} 8-\mathrm{N} 4-\mathrm{H} 2 \mathrm{~N}$

$\mathrm{H} 1 \mathrm{~N}-\mathrm{N} 4-\mathrm{H} 2 \mathrm{~N}$

$\mathrm{C} 11-\mathrm{N} 5-\mathrm{S} 2$

$\mathrm{C} 11-\mathrm{N} 5-\mathrm{K} 1$

$\mathrm{S} 2-\mathrm{N} 5-\mathrm{K} 1$

$\mathrm{C} 12-\mathrm{N} 6-\mathrm{C} 11$

$\mathrm{C} 12-\mathrm{N} 6-\mathrm{K} 2^{\mathrm{iii}}$

C11-N6-K2 $2^{\mathrm{iii}}$

C14-N7-C11

C14-N7-K1

C11-N7-K1

C18-N8-H3N

C18-N8-H4N

$\mathrm{H} 3 \mathrm{~N}-\mathrm{N} 8-\mathrm{H} 4 \mathrm{~N}$

$\mathrm{N} 2-\mathrm{C} 1-\mathrm{N} 3$

$\mathrm{N} 2-\mathrm{C} 1-\mathrm{N} 1$

N3- $1-\mathrm{N} 1$

$\mathrm{N} 2-\mathrm{C} 2-\mathrm{C} 3$

$\mathrm{N} 2-\mathrm{C} 2-\mathrm{H} 2$

$\mathrm{C} 3-\mathrm{C} 2-\mathrm{H} 2$

$\mathrm{C} 2-\mathrm{C} 3-\mathrm{C} 4$

$\mathrm{C} 2-\mathrm{C} 3-\mathrm{H} 3$

$\mathrm{C} 4-\mathrm{C} 3-\mathrm{H} 3$

N3- C4-C3

$\mathrm{N} 3-\mathrm{C} 4-\mathrm{H} 4$

$\mathrm{C} 3-\mathrm{C} 4-\mathrm{H} 4$

$\mathrm{C} 6-\mathrm{C} 5-\mathrm{C} 10$

C6- 5 - $-\mathrm{S} 1$

$\mathrm{C} 10-\mathrm{C} 5-\mathrm{S} 1$

$\mathrm{C} 5-\mathrm{C} 6-\mathrm{C} 7$

$\mathrm{C} 5-\mathrm{C} 6-\mathrm{H} 6$

$\mathrm{C} 7-\mathrm{C} 6-\mathrm{H} 6$

$\mathrm{C} 8-\mathrm{C} 7-\mathrm{C} 6$

$\mathrm{C} 8-\mathrm{C} 7-\mathrm{H} 7$
149 (4)

71 (3)

101 (2)

134 (5)

121 (7)

99 (2)

$94(8)$

99 (2)

120.6 (3)

139.9 (3)

$95.26(15)$

116.9 (4)

116.1 (4)

117 (4)

118 (4)

$110(6)$

122.3 (3)

91.7 (2)

138.42 (19)

116.1 (4)

$101.5(3)$

$123.5(3)$

116.3 (4)

$134.6(3)$

101.0 (3)

$121(3)$

120 (4)

$117(5)$

124.9 (4)

113.4 (4)

121.7 (4)

$122.6(5)$

118.7

118.7

$116.6(4)$

121.7

121.7

$122.8(5)$

118.6

118.6

119.2 (4)

$121.0(3)$

119.7 (4)

120.2 (4)

119.9

119.9

$121.2(5)$

119.4 
$\mathrm{O} 4 \mathrm{~W}-\mathrm{K} 2-\mathrm{S} 2$

$\mathrm{O} 3 \mathrm{~W}-\mathrm{K} 2-\mathrm{S} 2$

$\mathrm{O} 1-\mathrm{K} 2-\mathrm{S} 2$

$\mathrm{N} 6{ }^{\mathrm{iii}}-\mathrm{K} 2-\mathrm{S} 2$

$\mathrm{O} 4-\mathrm{K} 2-\mathrm{S} 2$

$\mathrm{O} 3-\mathrm{K} 2-\mathrm{S} 2$

$\mathrm{N} 1-\mathrm{K} 2-\mathrm{S} 2$

$\mathrm{O} 3^{\mathrm{iii}}-\mathrm{K} 2-\mathrm{C} 12^{\mathrm{iii}}$

$\mathrm{O} 4 \mathrm{~W}-\mathrm{K} 2-\mathrm{C} 12^{\mathrm{iii}}$

$\mathrm{O} 3 \mathrm{~W}-\mathrm{K} 2-\mathrm{C} 12^{\mathrm{iii}}$

$\mathrm{O} 1-\mathrm{K} 2-\mathrm{C} 12^{\mathrm{iii}}$

$\mathrm{N} 6{ }^{\mathrm{iii}}-\mathrm{K} 2-\mathrm{C} 12^{\mathrm{iii}}$

$\mathrm{O} 4-\mathrm{K} 2-\mathrm{C} 12^{\mathrm{iii}}$

$\mathrm{O} 3-\mathrm{K} 2-\mathrm{C} 12^{\mathrm{iii}}$

$\mathrm{N} 1-\mathrm{K} 2-\mathrm{C} 12^{\mathrm{iii}}$

$\mathrm{S} 2-\mathrm{K} 2-\mathrm{C} 12^{\mathrm{iii}}$

$\mathrm{O} 3{ }^{\mathrm{iii}}-\mathrm{K} 2-\mathrm{S} 1$

$\mathrm{O} 4 \mathrm{~W}-\mathrm{K} 2-\mathrm{S} 1$

$\mathrm{O} 3 \mathrm{~W}-\mathrm{K} 2-\mathrm{S} 1$

$\mathrm{O} 1-\mathrm{K} 2-\mathrm{S} 1$

$\mathrm{N} 6{ }^{\mathrm{iii}}-\mathrm{K} 2-\mathrm{S} 1$

$\mathrm{O} 4-\mathrm{K} 2-\mathrm{S} 1$

$\mathrm{O} 3-\mathrm{K} 2-\mathrm{S} 1$

$\mathrm{N} 1-\mathrm{K} 2-\mathrm{S} 1$

$\mathrm{S} 2-\mathrm{K} 2-\mathrm{S} 1$

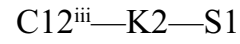

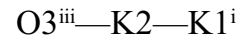

$\mathrm{O} 4 \mathrm{~W}-\mathrm{K} 2-\mathrm{K} 1^{\mathrm{i}}$

$\mathrm{O} 3 \mathrm{~W}-\mathrm{K} 2-\mathrm{K}^{\mathrm{i}}$

$\mathrm{O} 1-\mathrm{K} 2-\mathrm{K} 1^{\mathrm{i}}$

$\mathrm{N} 6{ }^{\mathrm{iii}}-\mathrm{K} 2-\mathrm{K} 1^{\mathrm{i}}$

$\mathrm{O} 4-\mathrm{K} 2-\mathrm{K} 1^{\mathrm{i}}$

$\mathrm{O} 3-\mathrm{K} 2-\mathrm{K} 1^{\mathrm{i}}$

$\mathrm{N} 1-\mathrm{K} 2-\mathrm{K} 1^{\mathrm{i}}$

$\mathrm{S} 2-\mathrm{K} 2-\mathrm{K} 1^{\mathrm{i}}$

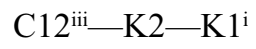

$\mathrm{S} 1-\mathrm{K} 2-\mathrm{K} 1^{\mathrm{i}}$

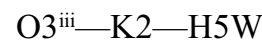

$\mathrm{O} 4 \mathrm{~W}-\mathrm{K} 2-\mathrm{H} 5 \mathrm{~W}$

$\mathrm{O} 3 \mathrm{~W}-\mathrm{K} 2-\mathrm{H} 5 \mathrm{~W}$

$\mathrm{O} 1-\mathrm{K} 2-\mathrm{H} 5 \mathrm{~W}$

$\mathrm{N} 6{ }^{\mathrm{iii}}-\mathrm{K} 2-\mathrm{H} 5 \mathrm{~W}$

$\mathrm{O} 4-\mathrm{K} 2-\mathrm{H} 5 \mathrm{~W}$

$\mathrm{O} 3-\mathrm{K} 2-\mathrm{H} 5 \mathrm{~W}$

$\mathrm{N} 1-\mathrm{K} 2-\mathrm{H} 5 \mathrm{~W}$

$\mathrm{S} 2-\mathrm{K} 2-\mathrm{H} 5 \mathrm{~W}$

$\mathrm{O} 2-\mathrm{S} 1-\mathrm{O} 1-\mathrm{K} 2$
84.25 (10)

85.87 (7)

$143.14(7)$

$126.55(7)$

$25.39(6)$

$25.46(6)$

$139.86(8)$

$86.35(10)$

$61.29(12)$

$136.33(10)$

$62.11(10)$

$22.46(10)$

$132.77(10)$

114.98 (9)

$85.10(10)$

$135.02(8)$

113.47 (7)

$101.02(10)$

$69.16(7)$

$22.03(6)$

$79.88(7)$

$131.84(6)$

$176.98(7)$

$25.63(7)$

153.19 (4)

$67.56(8)$

140.99 (7)

$81.88(9)$

$45.08(7)$

$82.84(7)$

$153.33(8)$

$39.76(6)$

$86.84(6)$

$94.45(7)$

$61.95(3)$

$131.72(8)$

$92.52(3)$

$111.4(10)$

$130.2(11)$

$17.7(2)$

$65.1(9)$

$128.6(3)$

93.3 (2)

$123.2(7)$

39.1 (3)

$102.5(4)$

$-107.96(19)$
$\mathrm{C} 6-\mathrm{C} 7-\mathrm{H} 7$

$\mathrm{C} 7-\mathrm{C} 8-\mathrm{C} 9$

$\mathrm{C} 7-\mathrm{C} 8-\mathrm{N} 4$

$\mathrm{C} 9-\mathrm{C} 8-\mathrm{N} 4$

$\mathrm{C} 8-\mathrm{C} 9-\mathrm{C} 10$

C8- 9 - $-\mathrm{H} 9$

$\mathrm{C} 10-\mathrm{C} 9-\mathrm{H} 9$

$\mathrm{C} 9-\mathrm{C} 10-\mathrm{C} 5$

$\mathrm{C} 9-\mathrm{C} 10-\mathrm{H} 10$

$\mathrm{C} 5-\mathrm{C} 10-\mathrm{H} 10$

N6- $111-\mathrm{N} 7$

$\mathrm{N} 6-\mathrm{C} 11-\mathrm{N} 5$

N7-C11-N5

$\mathrm{N} 6-\mathrm{C} 11-\mathrm{K} 1$

N7- C11-K1

N5-C11-K1

$\mathrm{N} 6-\mathrm{C} 12-\mathrm{C} 13$

$\mathrm{N} 6-\mathrm{C} 12-\mathrm{K}^{\mathrm{iii}}$

$\mathrm{C} 13-\mathrm{C} 12-\mathrm{K}^{\mathrm{iii}}$

$\mathrm{N} 6-\mathrm{C} 12-\mathrm{H} 12$

$\mathrm{C} 13-\mathrm{C} 12-\mathrm{H} 12$

$\mathrm{K} 2{ }^{\mathrm{iii}}-\mathrm{C} 12-\mathrm{H} 12$

$\mathrm{C} 14-\mathrm{C} 13-\mathrm{C} 12$

$\mathrm{C} 14-\mathrm{C} 13-\mathrm{H} 13$

$\mathrm{C} 12-\mathrm{C} 13-\mathrm{H} 13$

$\mathrm{N} 7-\mathrm{C} 14-\mathrm{C} 13$

N7-C14-H14

C13-C14-H14

$\mathrm{C} 20-\mathrm{C} 15-\mathrm{C} 16$

$\mathrm{C} 20-\mathrm{C} 15-\mathrm{S} 2$

$\mathrm{C} 16-\mathrm{C} 15-\mathrm{S} 2$

$\mathrm{C} 17-\mathrm{C} 16-\mathrm{C} 15$

C17-C16-H16

$\mathrm{C} 15-\mathrm{C} 16-\mathrm{H} 16$

$\mathrm{C} 16-\mathrm{C} 17-\mathrm{C} 18$

C16-C17-H17

C18-C17-H17

N8-C18-C19

$\mathrm{N} 8-\mathrm{C} 18-\mathrm{C} 17$

$\mathrm{C} 19-\mathrm{C} 18-\mathrm{C} 17$

$\mathrm{C} 20-\mathrm{C} 19-\mathrm{C} 18$

C20-C19-H19

C18-C19-H19

$\mathrm{C} 19-\mathrm{C} 20-\mathrm{C} 15$

$\mathrm{C} 19-\mathrm{C} 20-\mathrm{H} 20$

$\mathrm{C} 15-\mathrm{C} 20-\mathrm{H} 20$

$\mathrm{C} 10-\mathrm{C} 5-\mathrm{C} 6-\mathrm{C} 7$
119.4

118.4 (4)

$120.9(5)$

120.7 (5)

$121.0(4)$

119.5

119.5

119.9 (5)

120.1

120.1

$124.8(4)$

$121.6(4)$

$113.6(4)$

$154.2(3)$

$56.4(2)$

$64.8(2)$

123.7 (4)

$56.0(2)$

143.9 (3)

118.2

118.2

74.7

115.2 (4)

122.4

122.4

123.8 (5)

118.1

118.1

120.1 (4)

120.3 (3)

$119.6(3)$

120.0 (4)

120.0

120.0

121.0 (4)

119.5

119.5

120.3 (4)

121.9 (4)

117.7 (4)

$121.6(4)$

119.2

119.2

119.5 (4)

120.2

120.2

$0.0(7)$ 
$\mathrm{N} 1-\mathrm{S} 1-\mathrm{O} 1-\mathrm{K} 2$

$\mathrm{C} 5-\mathrm{S} 1-\mathrm{O} 1-\mathrm{K} 2$

$\mathrm{O} 4-\mathrm{S} 2-\mathrm{O} 3-\mathrm{K} 2^{\mathrm{iii}}$

$\mathrm{N} 5-\mathrm{S} 2-\mathrm{O} 3-\mathrm{K} 2^{\mathrm{iii}}$

$\mathrm{C} 15-\mathrm{S} 2-\mathrm{O} 3-\mathrm{K} 2^{\mathrm{iii}}$

$\mathrm{K} 2-\mathrm{S} 2-\mathrm{O} 3-\mathrm{K} 2{ }^{\mathrm{iii}}$

$\mathrm{O} 4-\mathrm{S} 2-\mathrm{O} 3-\mathrm{K} 2$

$\mathrm{N} 5-\mathrm{S} 2-\mathrm{O} 3-\mathrm{K} 2$

$\mathrm{C} 15-\mathrm{S} 2-\mathrm{O} 3-\mathrm{K} 2$

$\mathrm{O} 3-\mathrm{S} 2-\mathrm{O} 4-\mathrm{K}^{\mathrm{i}}$

$\mathrm{N} 5-\mathrm{S} 2-\mathrm{O} 4-\mathrm{K}^{\mathrm{i}}$

$\mathrm{C} 15-\mathrm{S} 2-\mathrm{O} 4-\mathrm{K} 1^{\mathrm{i}}$

$\mathrm{K} 2-\mathrm{S} 2-\mathrm{O} 4-\mathrm{K}^{1}$

$\mathrm{O} 3-\mathrm{S} 2-\mathrm{O} 4-\mathrm{K} 2$

$\mathrm{N} 5-\mathrm{S} 2-\mathrm{O} 4-\mathrm{K} 2$

$\mathrm{C} 15-\mathrm{S} 2-\mathrm{O} 4-\mathrm{K} 2$

$\mathrm{O} 2-\mathrm{S} 1-\mathrm{N} 1-\mathrm{C} 1$

$\mathrm{O} 1-\mathrm{S} 1-\mathrm{N} 1-\mathrm{C} 1$

$\mathrm{C} 5-\mathrm{S} 1-\mathrm{N} 1-\mathrm{C} 1$

$\mathrm{K} 2-\mathrm{S} 1-\mathrm{N} 1-\mathrm{C} 1$

$\mathrm{O} 2-\mathrm{S} 1-\mathrm{N} 1-\mathrm{K} 2$

$\mathrm{O} 1-\mathrm{S} 1-\mathrm{N} 1-\mathrm{K} 2$

$\mathrm{C} 5-\mathrm{S} 1-\mathrm{N} 1-\mathrm{K} 2$

$\mathrm{O} 3-\mathrm{S} 2-\mathrm{N} 5-\mathrm{C} 11$

$\mathrm{O} 4-\mathrm{S} 2-\mathrm{N} 5-\mathrm{C} 11$

$\mathrm{C} 15-\mathrm{S} 2-\mathrm{N} 5-\mathrm{C} 11$

$\mathrm{K} 2-\mathrm{S} 2-\mathrm{N} 5-\mathrm{C} 11$

$\mathrm{O} 3-\mathrm{S} 2-\mathrm{N} 5-\mathrm{K} 1$

$\mathrm{O} 4-\mathrm{S} 2-\mathrm{N} 5-\mathrm{K} 1$

$\mathrm{C} 15-\mathrm{S} 2-\mathrm{N} 5-\mathrm{K} 1$

$\mathrm{K} 2-\mathrm{S} 2-\mathrm{N} 5-\mathrm{K} 1$

$\mathrm{C} 2-\mathrm{N} 2-\mathrm{C} 1-\mathrm{N} 3$

$\mathrm{C} 2-\mathrm{N} 2-\mathrm{C} 1-\mathrm{N} 1$

$\mathrm{C} 4-\mathrm{N} 3-\mathrm{C} 1-\mathrm{N} 2$

$\mathrm{C} 4-\mathrm{N} 3-\mathrm{C} 1-\mathrm{N} 1$

$\mathrm{S} 1-\mathrm{N} 1-\mathrm{C} 1-\mathrm{N} 2$

$\mathrm{K} 2-\mathrm{N} 1-\mathrm{C} 1-\mathrm{N} 2$

$\mathrm{S} 1-\mathrm{N} 1-\mathrm{C} 1-\mathrm{N} 3$

$\mathrm{K} 2-\mathrm{N} 1-\mathrm{C} 1-\mathrm{N} 3$

$\mathrm{C} 1-\mathrm{N} 2-\mathrm{C} 2-\mathrm{C} 3$

$\mathrm{N} 2-\mathrm{C} 2-\mathrm{C} 3-\mathrm{C} 4$

$\mathrm{C} 1-\mathrm{N} 3-\mathrm{C} 4-\mathrm{C} 3$

$\mathrm{C} 2-\mathrm{C} 3-\mathrm{C} 4-\mathrm{N} 3$

$\mathrm{O} 2-\mathrm{S} 1-\mathrm{C} 5-\mathrm{C} 6$

$\mathrm{O} 1-\mathrm{S} 1-\mathrm{C} 5-\mathrm{C} 6$

$\mathrm{N} 1-\mathrm{S} 1-\mathrm{C} 5-\mathrm{C} 6$

$\mathrm{K} 2-\mathrm{S} 1-\mathrm{C} 5-\mathrm{C} 6$

$\mathrm{O} 2-\mathrm{S} 1-\mathrm{C} 5-\mathrm{C} 10$
$18.6(2)$

$132.83(18)$

$-161.4(2)$

-40.7 (3)

81.7 (3)

$-126.1(2)$

$-35.30(18)$

$85.46(17)$

$-152.14(15)$

143.5 (3)

17.7 (3)

$-99.4(3)$

$107.0(3)$

36.46 (19)

$-89.27(17)$

$153.58(16)$

$-52.2(4)$

$-177.3(3)$

$69.1(3)$

$-161.3(4)$

$109.16(17)$

$-15.96(17)$

$-129.60(16)$

54.6 (4)

$179.4(3)$

$-66.0(4)$

$118.3(3)$

$-85.3(3)$

39.5 (3)

154.1 (2)

$-21.6(3)$

$4.1(6)$

$-175.5(4)$

$-4.1(6)$

$175.5(4)$

$175.3(3)$

$25.0(6)$

$-4.4(5)$

$-154.7(3)$

$0.0(7)$

$-3.6(7)$

$-0.1(6)$

3.7 (7)

$143.5(4)$

$-94.0(4)$

17.7 (4)

-46.4 (4)

-39.9 (4)
$\mathrm{S} 1-\mathrm{C} 5-\mathrm{C} 6-\mathrm{C} 7$

$\mathrm{C} 5-\mathrm{C} 6-\mathrm{C} 7-\mathrm{C} 8$

C6- $7-\mathrm{C} 8-\mathrm{C} 9$

$\mathrm{C} 6-\mathrm{C} 7-\mathrm{C} 8-\mathrm{N} 4$

$\mathrm{C} 7-\mathrm{C} 8-\mathrm{C} 9-\mathrm{C} 10$

$\mathrm{N} 4-\mathrm{C} 8-\mathrm{C} 9-\mathrm{C} 10$

$\mathrm{C} 8-\mathrm{C} 9-\mathrm{C} 10-\mathrm{C} 5$

$\mathrm{C} 6-\mathrm{C} 5-\mathrm{C} 10-\mathrm{C} 9$

$\mathrm{S} 1-\mathrm{C} 5-\mathrm{C} 10-\mathrm{C} 9$

C12-N6-C11-N7

$\mathrm{K} 2{ }^{i i i}-\mathrm{N} 6-\mathrm{C} 11-\mathrm{N} 7$

$\mathrm{C} 12-\mathrm{N} 6-\mathrm{C} 11-\mathrm{N} 5$

$\mathrm{K} 2{ }^{\mathrm{iii}}-\mathrm{N} 6-\mathrm{C} 11-\mathrm{N} 5$

$\mathrm{C} 12-\mathrm{N} 6-\mathrm{C} 11-\mathrm{K} 1$

$\mathrm{K} 2{ }^{\mathrm{iii}}-\mathrm{N} 6-\mathrm{C} 11-\mathrm{K} 1$

C14-N7-C11-N6

$\mathrm{K} 1-\mathrm{N} 7-\mathrm{C} 11-\mathrm{N} 6$

$\mathrm{C} 14-\mathrm{N} 7-\mathrm{C} 11-\mathrm{N} 5$

$\mathrm{K} 1-\mathrm{N} 7-\mathrm{C} 11-\mathrm{N} 5$

$\mathrm{C} 14-\mathrm{N} 7-\mathrm{C} 11-\mathrm{K} 1$

$\mathrm{S} 2-\mathrm{N} 5-\mathrm{C} 11-\mathrm{N} 6$

$\mathrm{K} 1-\mathrm{N} 5-\mathrm{C} 11-\mathrm{N} 6$

$\mathrm{S} 2-\mathrm{N} 5-\mathrm{C} 11-\mathrm{N} 7$

$\mathrm{K} 1-\mathrm{N} 5-\mathrm{C} 11-\mathrm{N} 7$

S2-N5-C11-K1

$\mathrm{C} 11-\mathrm{N} 6-\mathrm{C} 12-\mathrm{C} 13$

$\mathrm{K} 2{ }^{\mathrm{iii}}-\mathrm{N} 6-\mathrm{C} 12-\mathrm{C} 13$

$\mathrm{C} 11-\mathrm{N} 6-\mathrm{C} 12-\mathrm{K}^{\mathrm{iii}}$

$\mathrm{N} 6-\mathrm{C} 12-\mathrm{C} 13-\mathrm{C} 14$

$\mathrm{K} 2{ }^{\mathrm{iii}}-\mathrm{C} 12-\mathrm{C} 13-\mathrm{C} 14$

C11-N7-C14-C13

$\mathrm{K} 1-\mathrm{N} 7-\mathrm{C} 14-\mathrm{C} 13$

$\mathrm{C} 12-\mathrm{C} 13-\mathrm{C} 14-\mathrm{N} 7$

$\mathrm{O} 3-\mathrm{S} 2-\mathrm{C} 15-\mathrm{C} 20$

$\mathrm{O} 4-\mathrm{S} 2-\mathrm{C} 15-\mathrm{C} 20$

N5-S2-C15-C20

$\mathrm{K} 2-\mathrm{S} 2-\mathrm{C} 15-\mathrm{C} 20$

$\mathrm{O} 3-\mathrm{S} 2-\mathrm{C} 15-\mathrm{C} 16$

$\mathrm{O} 4-\mathrm{S} 2-\mathrm{C} 15-\mathrm{C} 16$

N5-S2-C15-C16

$\mathrm{K} 2-\mathrm{S} 2-\mathrm{C} 15-\mathrm{C} 16$

$\mathrm{C} 20-\mathrm{C} 15-\mathrm{C} 16-\mathrm{C} 17$

$\mathrm{S} 2-\mathrm{C} 15-\mathrm{C} 16-\mathrm{C} 17$

$\mathrm{C} 15-\mathrm{C} 16-\mathrm{C} 17-\mathrm{C} 18$

$\mathrm{C} 16-\mathrm{C} 17-\mathrm{C} 18-\mathrm{N} 8$

$\mathrm{C} 16-\mathrm{C} 17-\mathrm{C} 18-\mathrm{C} 19$

$\mathrm{N} 8-\mathrm{C} 18-\mathrm{C} 19-\mathrm{C} 20$

$\mathrm{C} 17-\mathrm{C} 18-\mathrm{C} 19-\mathrm{C} 20$
$176.6(3)$

$-1.8(7)$

$2.5(7)$

$179.0(4)$

$-1.4(7)$

$-178.0(5)$

$-0.3(8)$

$1.0(7)$

$-175.6(4)$

$-4.4(6)$

$121.7(4)$

$174.9(4)$

$-59.0(5)$

-88.0 (7)

$38.2(8)$

4.7 (7)

-148.8 (4)

-174.6 (4)

32.0 (4)

$153.4(5)$

$-3.2(6)$

151.5 (4)

$176.2(3)$

-29.2 (4)

-154.7 (3)

0.4 (6)

-136.1 (4)

136.5 (4)

2.8 (7)

$-74.1(7)$

$-1.0(7)$

140.9 (4)

$-2.5(7)$

-20.6 (4)

$-141.6(3)$

104.4 (4)

-85.0 (4)

160.5 (3)

39.5 (4)

-74.4 (4)

96.1 (4)

0.4 (6)

179.2 (3)

1.4 (7)

$176.3(4)$

$-1.8(7)$

-177.7 (4)

0.5 (6) 
$\mathrm{O} 1-\mathrm{S} 1-\mathrm{C} 5-\mathrm{C} 10$
$\mathrm{~N} 1-\mathrm{S} 1-\mathrm{C} 5-\mathrm{C} 10$
$\mathrm{~K} 2-\mathrm{S} 1-\mathrm{C} 5-\mathrm{C} 10$

$82.6(4)$

$-165.7(4)$
$1.3(6)$

$-1.7(6)$

$179.4(3)$

Symmetry codes: (i) $-x,-y+1,-z+1$; (ii) $-x+1,-y+1,-z+1$; (iii) $-x,-y+2,-z+1$.

Hydrogen-bond geometry $\left(\AA,{ }^{\circ}\right)$

\begin{tabular}{lllll}
\hline$D-\mathrm{H} \cdots A$ & $D-\mathrm{H}$ & $\mathrm{H} \cdots A$ & $D \cdots A$ & $D-\mathrm{H} \cdots A$ \\
\hline $\mathrm{O} 1 W-\mathrm{H} 1 W^{\cdots} \cdot \mathrm{O} 1^{\mathrm{i}}$ & $0.88(1)$ & $1.93(2)$ & $2.799(5)$ & $167(8)$ \\
$\mathrm{O} 1 W-\mathrm{H} 2 W \cdots \mathrm{O} 4 W^{\mathrm{i}}$ & $0.88(1)$ & $2.27(4)$ & $3.070(6)$ & $151(6)$ \\
$\mathrm{O} 2 W-\mathrm{H} 3 W \cdots \mathrm{O} 3^{\text {iii }}$ & $0.88(1)$ & $2.07(2)$ & $2.933(5)$ & $167(6)$ \\
$\mathrm{O} 2 W-\mathrm{H} 4 W \cdots \mathrm{N} 2$ & $0.88(1)$ & $1.97(2)$ & $2.828(5)$ & $167(6)$ \\
$\mathrm{O} 3 W-\mathrm{H} 5 W \cdots \mathrm{N} 1$ & $0.87(1)$ & $2.02(2)$ & $2.872(5)$ & $164(5)$ \\
$\mathrm{O} 3 W-\mathrm{H} 6 W \cdots \mathrm{N} 5^{\mathrm{i}}$ & $0.87(1)$ & $1.98(1)$ & $2.835(5)$ & $166(4)$ \\
$\mathrm{O} 4 W-\mathrm{H} 7 W \cdots \mathrm{O} 2 W^{\text {iv }}$ & $0.88(1)$ & $2.05(2)$ & $2.919(6)$ & $169(7)$ \\
$\mathrm{O} 4 W-\mathrm{H} 8 W \cdots \mathrm{O} 4 W^{v}$ & $0.88(1)$ & $2.05(2)$ & $2.920(8)$ & $168(9)$ \\
$\mathrm{N} 4-\mathrm{H} 1 N \cdots \mathrm{N} 4^{\text {vi }}$ & $0.87(7)$ & $2.50(6)$ & $3.054(9)$ & $122(5)$ \\
$\mathrm{N} 4-\mathrm{H} 2 N \cdots \mathrm{O} 2^{\text {vii }}$ & $0.93(7)$ & $2.57(7)$ & $3.431(7)$ & $153(5)$ \\
$\mathrm{N} 8-\mathrm{H} 3 N \cdots \mathrm{O} 2^{\text {viii }}$ & $0.87(5)$ & $2.38(5)$ & $3.046(5)$ & $134(4)$ \\
$\mathrm{N} 8-\mathrm{H} 3 N \cdots \mathrm{N} 3^{\text {viii }}$ & $0.87(5)$ & $2.61(5)$ & $3.283(6)$ & $135(4)$
\end{tabular}

Symmetry codes: (i) $-x,-y+1,-z+1$; (iii) $-x,-y+2,-z+1$; (iv) $x-1, y, z$; (v) $-x-1,-y+2,-z+1$; (vi) $-x-1,-y,-z$; (vii) $x, y-1, z$; (viii) $x, y, z+1$.

(IV)

Crystal data

$\mathrm{C}_{26} \mathrm{H}_{32} \mathrm{~N}_{6} \mathrm{NaO}_{14.50} \mathrm{~S}_{3}$

$M_{r}=779.74$

Monoclinic, $P 2_{1} / c$

$a=12.7688(1) \AA$

$b=16.9040(1) \AA$

$c=15.7411(1) \AA$

$\beta=107.609(1)^{\circ}$

$V=3238.42(4) \AA^{3}$

$Z=4$

Data collection

\section{XtaLAB AFC11 (RCD3)} diffractometer

Radiation source: rotating anode $\omega$ scans

Absorption correction: multi-scan

CrysAlis PRO 1.171.39.34b (Rigaku Oxford

Diffraction, 2017) Empirical absorption

correction using spherical harmonics,

implemented in SCALE3 ABSPACK scaling algorithm.

\section{Refinement}

Refinement on $F^{2}$

Least-squares matrix: full

$R\left[F^{2}>2 \sigma\left(F^{2}\right)\right]=0.028$
$F(000)=1620$

$D_{\mathrm{x}}=1.599 \mathrm{Mg} \mathrm{m}^{-3}$

$\mathrm{Cu} K \alpha$ radiation, $\lambda=1.54184 \AA$

Cell parameters from 35404 reflections

$\theta=3.6-70.3^{\circ}$

$\mu=2.95 \mathrm{~mm}^{-1}$

$T=100 \mathrm{~K}$

Block, red

$0.12 \times 0.08 \times 0.04 \mathrm{~mm}$

$T_{\min }=0.615, T_{\max }=1.000$

59125 measured reflections

5913 independent reflections

5686 reflections with $I>2 \sigma(I)$

$R_{\text {int }}=0.027$

$\theta_{\max }=68.3^{\circ}, \theta_{\min }=3.6^{\circ}$

$h=-15 \rightarrow 15$

$k=-20 \rightarrow 20$

$l=-18 \rightarrow 17$

$w R\left(F^{2}\right)=0.075$

$S=1.05$

5913 reflections 
537 parameters

13 restraints

Hydrogen site location: mixed

$\mathrm{H}$ atoms treated by a mixture of independent

and constrained refinement

$$
\begin{aligned}
& w=1 /\left[\sigma^{2}\left(F_{\mathrm{o}}^{2}\right)+(0.0364 P)^{2}+2.347 P\right] \\
& \text { where } P=\left(F_{\mathrm{o}}^{2}+2 F_{\mathrm{c}}{ }^{2}\right) / 3 \\
& (\Delta / \sigma)_{\max }=0.001 \\
& \Delta \rho_{\max }=0.26 \mathrm{e} \AA^{-3} \\
& \Delta \rho_{\min }=-0.39 \mathrm{e} \AA^{-3}
\end{aligned}
$$

Special details

Geometry. All e.s.d.'s (except the e.s.d. in the dihedral angle between two 1.s. planes) are estimated using the full

\begin{tabular}{|c|c|c|c|c|c|}
\hline & $x$ & $y$ & $z$ & $U_{\text {iso }} * / U_{\text {eq }}$ & Occ. $(<1)$ \\
\hline $\mathrm{Na} 1$ & $-0.36434(5)$ & $0.35305(4)$ & $0.00412(4)$ & $0.02103(14)$ & \\
\hline $\mathrm{S} 1$ & $0.25698(3)$ & $0.53159(2)$ & $0.56811(2)$ & $0.01894(9)$ & \\
\hline $\mathrm{S} 2$ & $0.28977(3)$ & $0.35439(2)$ & $0.12953(2)$ & $0.01723(9)$ & \\
\hline $\mathrm{S} 3$ & $0.34948(3)$ & $0.66079(2)$ & $0.22267(2)$ & $0.01823(9)$ & \\
\hline $\mathrm{O} 1$ & $0.30756(9)$ & $0.60882(7)$ & $0.58033(7)$ & $0.0231(2)$ & \\
\hline $\mathrm{O} 2$ & $0.25734(9)$ & $0.48924(7)$ & $0.64777(7)$ & $0.0232(2)$ & \\
\hline $\mathrm{O} 1 \mathrm{~W}$ & $-0.35372(9)$ & $0.22020(7)$ & $0.04238(8)$ & $0.0218(2)$ & \\
\hline $\mathrm{O} 3$ & $-0.16759(8)$ & $0.36126(6)$ & $0.04200(7)$ & $0.0209(2)$ & \\
\hline $\mathrm{O} 2 \mathrm{~W}$ & $-0.39276(13)$ & $0.36471(9)$ & $0.15242(9)$ & $0.0366(3)$ & \\
\hline $\mathrm{O} 4$ & $0.23184(9)$ & $0.28982(6)$ & $0.15504(8)$ & $0.0242(2)$ & \\
\hline $\mathrm{O} 3 \mathrm{~W}$ & $-0.56243(11)$ & $0.33362(8)$ & $-0.06717(10)$ & $0.0324(3)$ & \\
\hline $\mathrm{O} 5$ & $0.29466(9)$ & $0.35183(7)$ & $0.03839(7)$ & $0.0247(3)$ & \\
\hline O4W & $-0.40375(13)$ & $0.48626(9)$ & $-0.02427(12)$ & $0.0467(4)$ & \\
\hline H7W & $-0.3673(17)$ & $0.5308(9)$ & $-0.0208(17)$ & $0.056^{*}$ & \\
\hline H8W & $-0.449(3)$ & $0.501(2)$ & $0.006(3)$ & $0.056^{*}$ & 0.5 \\
\hline H14W & $-0.463(2)$ & $0.499(2)$ & $-0.068(2)$ & $0.056^{*}$ & 0.5 \\
\hline O5W & $-0.6489(2)$ & $0.33037(15)$ & $-0.24880(18)$ & $0.0323(5)$ & 0.5 \\
\hline H9W & $-0.677(3)$ & $0.2873(16)$ & $-0.279(2)$ & $0.039 *$ & 0.5 \\
\hline H10W & $-0.590(2)$ & $0.336(2)$ & $-0.267(3)$ & $0.039 *$ & 0.5 \\
\hline O6W & -0.3558 & $0.52405(18)$ & $0.16139(18)$ & $0.0459(8)$ & 0.5 \\
\hline H11W & $-0.366(2)$ & $0.5317(15)$ & $0.2141(10)$ & $0.055^{*}$ & \\
\hline H12W & $-0.367(4)$ & $0.4725(8)$ & $0.160(3)$ & $0.055^{*}$ & 0.5 \\
\hline O7W & $-0.4311(2)$ & $0.54771(18)$ & $0.19266(18)$ & $0.0392(7)$ & 0.5 \\
\hline H13W & $-0.451(3)$ & $0.566(3)$ & $0.2374(18)$ & $0.047 *$ & 0.5 \\
\hline O6 & $0.40067(8)$ & $0.36455(6)$ & $0.19268(7)$ & $0.0200(2)$ & \\
\hline $\mathrm{O} 7$ & $0.36235(9)$ & $0.69334(7)$ & $0.14107(7)$ & $0.0228(2)$ & \\
\hline O8 & $0.44918(9)$ & $0.62573(7)$ & $0.28078(8)$ & $0.0297(3)$ & \\
\hline O9 & $0.29864(9)$ & $0.71689(7)$ & 0.26880 & $0.0241(2)$ & \\
\hline N1 & $0.13018(10)$ & $0.53245(8)$ & $0.50886(8)$ & 0.0193 & \\
\hline N2 & $0.14382(11)$ & $0.63967(8)$ & $0.41387(9)$ & $0.0205(3)$ & \\
\hline N3 & $-0.02496(10)$ & $0.58329(8)$ & $0.41174(9)$ & $0.0202(3)$ & \\
\hline N4 & $0.47872(12)$ & 0.31848 (9) & $0.37342(10)$ & 0.0228 & \\
\hline N5 & $0.04786(10)$ & $0.34343(7)$ & $0.02685(8)$ & 0.0165 & \\
\hline N6 & $-0.02618(10)$ & $0.29254(7)$ & $-0.01560(8)$ & 0.0173 & \\
\hline
\end{tabular}
covariance matrix. The cell e.s.d.'s are taken into account individually in the estimation of e.s.d.'s in distances, angles and torsion angles; correlations between e.s.d.'s in cell parameters are only used when they are defined by crystal symmetry. An approximate (isotropic) treatment of cell e.s.d.'s is used for estimating e.s.d.'s involving 1.s. planes.

Fractional atomic coordinates and isotropic or equivalent isotropic displacement parameters $\left(\AA^{2}\right)$ 


\begin{tabular}{|c|c|c|c|c|c|}
\hline 824 & $\mathrm{C} 1$ & $0.08718(12)$ & $0.58593(9)$ & $0.44525(10)$ & $0.0185(3)$ \\
\hline 825 & $\mathrm{C} 2$ & $0.08701(13)$ & $0.69124(10)$ & $0.35332(10)$ & $0.0216(3)$ \\
\hline 826 & $\mathrm{H} 2$ & 0.1270 & 0.7293 & 0.3313 & $0.026^{*}$ \\
\hline 827 & $\mathrm{C} 3$ & $-0.02726(13)$ & $0.69278(10)$ & $0.32031(11)$ & $0.0232(3)$ \\
\hline 828 & $\mathrm{H} 3$ & -0.0650 & 0.7314 & 0.2784 & $0.028 *$ \\
\hline 829 & $\mathrm{C} 4$ & -0.08209 (13) & $0.63587(10)$ & $0.35134(11)$ & $0.0238(3)$ \\
\hline 830 & $\mathrm{H} 4$ & -0.1600 & 0.6334 & 0.3304 & $0.029 *$ \\
\hline 831 & C5 & $0.32606(12)$ & $0.47095(9)$ & $0.51081(10)$ & $0.0197(3)$ \\
\hline 832 & C6 & $0.35300(12)$ & $0.49962(10)$ & $0.43696(10)$ & $0.0223(3)$ \\
\hline 833 & H6 & 0.3365 & 0.5527 & 0.4176 & $0.027^{*}$ \\
\hline 834 & $\mathrm{C} 7$ & $0.40425(12)$ & $0.44943(10)$ & $0.39231(10)$ & $0.0219(3)$ \\
\hline 835 & $\mathrm{H} 7$ & 0.4236 & 0.4677 & 0.3420 & $0.026^{*}$ \\
\hline 836 & $\mathrm{C} 8$ & $0.42703(12)$ & $0.37216(10)$ & $0.42193(10)$ & $0.0205(3)$ \\
\hline 837 & C9 & $0.40333(13)$ & $0.34437(10)$ & $0.49661(11)$ & $0.0221(3)$ \\
\hline 838 & H9 & 0.4223 & 0.2919 & 0.5171 & $0.027^{*}$ \\
\hline 839 & $\mathrm{C} 10$ & $0.35154(12)$ & $0.39416(10)$ & $0.54122(10)$ & $0.0218(3)$ \\
\hline 840 & H10 & 0.3336 & 0.3759 & 0.5922 & $0.026^{*}$ \\
\hline 841 & C11 & 0.01948 (12) & $0.40012(9)$ & $0.07444(10)$ & $0.0159(3)$ \\
\hline 842 & $\mathrm{C} 12$ & $-0.09441(12)$ & $0.41261(9)$ & $0.07379(9)$ & $0.0175(3)$ \\
\hline 843 & C13 & $-0.12211(12)$ & $0.48735(9)$ & $0.10541(10)$ & $0.0198(3)$ \\
\hline 844 & $\mathrm{H} 13$ & -0.1955 & 0.4971 & 0.1051 & $0.024 *$ \\
\hline 845 & C14 & $-0.04523(12)$ & $0.54336(9)$ & $0.13538(10)$ & $0.0188(3)$ \\
\hline 846 & $\mathrm{H} 14$ & -0.0671 & 0.5934 & 0.1516 & $0.023 *$ \\
\hline 847 & $\mathrm{C} 15$ & $0.06970(12)$ & $0.53059(9)$ & $0.14404(10)$ & $0.0166(3)$ \\
\hline 848 & C16 & $0.10495(12)$ & $0.45694(9)$ & $0.11881(9)$ & $0.0157(3)$ \\
\hline 849 & C17 & $0.22061(12)$ & $0.44559(9)$ & $0.13890(10)$ & $0.0168(3)$ \\
\hline 850 & C18 & $0.29200(12)$ & $0.50782(9)$ & $0.17202(10)$ & $0.0186(3)$ \\
\hline 851 & H18 & 0.3687 & 0.4999 & 0.1835 & $0.022 *$ \\
\hline 852 & C19 & $0.25427(12)$ & $0.58168(9)$ & $0.18898(10)$ & 0.0178 \\
\hline 853 & $\mathrm{C} 20$ & $0.14397(12)$ & $0.59221(9)$ & $0.17777(10)$ & $0.0178(3)$ \\
\hline 854 & $\mathrm{H} 20$ & 0.1182 & 0.6413 & 0.1929 & $0.021 *$ \\
\hline 855 & $\mathrm{C} 21$ & $-0.00296(12)$ & $0.23542(9)$ & $-0.07255(10)$ & $0.0178(3)$ \\
\hline 856 & $\mathrm{C} 22$ & $-0.09205(13)$ & $0.19251(9)$ & $-0.12561(10)$ & $0.0204(3)$ \\
\hline 857 & $\mathrm{H} 22$ & -0.1643 & 0.2031 & -0.1234 & $0.024 *$ \\
\hline 858 & $\mathrm{C} 23$ & $-0.07414(14)$ & $0.13423(10)$ & $-0.18169(11)$ & $0.0237(3)$ \\
\hline 859 & $\mathrm{H} 23$ & -0.1343 & 0.1047 & -0.2182 & $0.028^{*}$ \\
\hline 860 & $\mathrm{C} 24$ & $0.03150(14)$ & $0.11899(10)$ & $-0.18464(11)$ & $0.0243(3)$ \\
\hline 861 & $\mathrm{H} 24$ & 0.0438 & 0.0786 & -0.2225 & $0.029 *$ \\
\hline 862 & $\mathrm{C} 25$ & $0.11952(14)$ & $0.16297(10)$ & $-0.13190(11)$ & $0.0242(3)$ \\
\hline 863 & $\mathrm{H} 25$ & 0.1916 & 0.1526 & -0.1346 & $0.029 *$ \\
\hline 864 & $\mathrm{C} 26$ & $0.10358(13)$ & $0.22174(10)$ & $-0.07551(10)$ & $0.0211(3)$ \\
\hline 865 & $\mathrm{H} 26$ & 0.1637 & 0.2519 & -0.0398 & $0.025^{*}$ \\
\hline 866 & $\mathrm{H} 1 \mathrm{~W}$ & $-0.349(2)$ & $0.2133(14)$ & $0.0986(18)$ & $0.049(7)^{*}$ \\
\hline 867 & $\mathrm{H} 2 \mathrm{~W}$ & $-0.299(2)$ & $0.1888(15)$ & $0.0354(16)$ & $0.052(7)^{*}$ \\
\hline 868 & $\mathrm{H} 3 \mathrm{~W}$ & $-0.360(2)$ & $0.3264(17)$ & $0.1861(18)$ & $0.057(8)^{*}$ \\
\hline 869 & $\mathrm{H} 4 \mathrm{~W}$ & $-0.461(3)$ & $0.3655(19)$ & $0.161(2)$ & $0.089(11)^{*}$ \\
\hline 870 & H5W & $-0.607(2)$ & 0.3395 (16) & $-0.0361(19)$ & $0.061(8)^{*}$ \\
\hline 871 & H6W & $-0.591(3)$ & $0.365(2)$ & $-0.109(2)$ & $0.086(11)^{*}$ \\
\hline
\end{tabular}




$\begin{array}{lllll}\text { H1N } & -0.0943(19) & 0.2989(13) & -0.0086(14) & 0.039(6)^{*} \\ \text { H2N } & 0.4668(19) & 0.2671(15) & 0.3875(16) & 0.046(6)^{*} \\ \text { H3N } & 0.4510(18) & 0.3275(13) & 0.3137(16) & 0.036(6)^{*} \\ \text { H4N } & 0.552(2) & 0.3289(13) & 0.3887(14) & 0.036(6)^{*} \\ \text { H5N } & -0.0568(18) & 0.5484(13) & 0.4332(14) & 0.034(6)^{*}\end{array}$

Atomic displacement parameters $\left(\AA^{2}\right)$

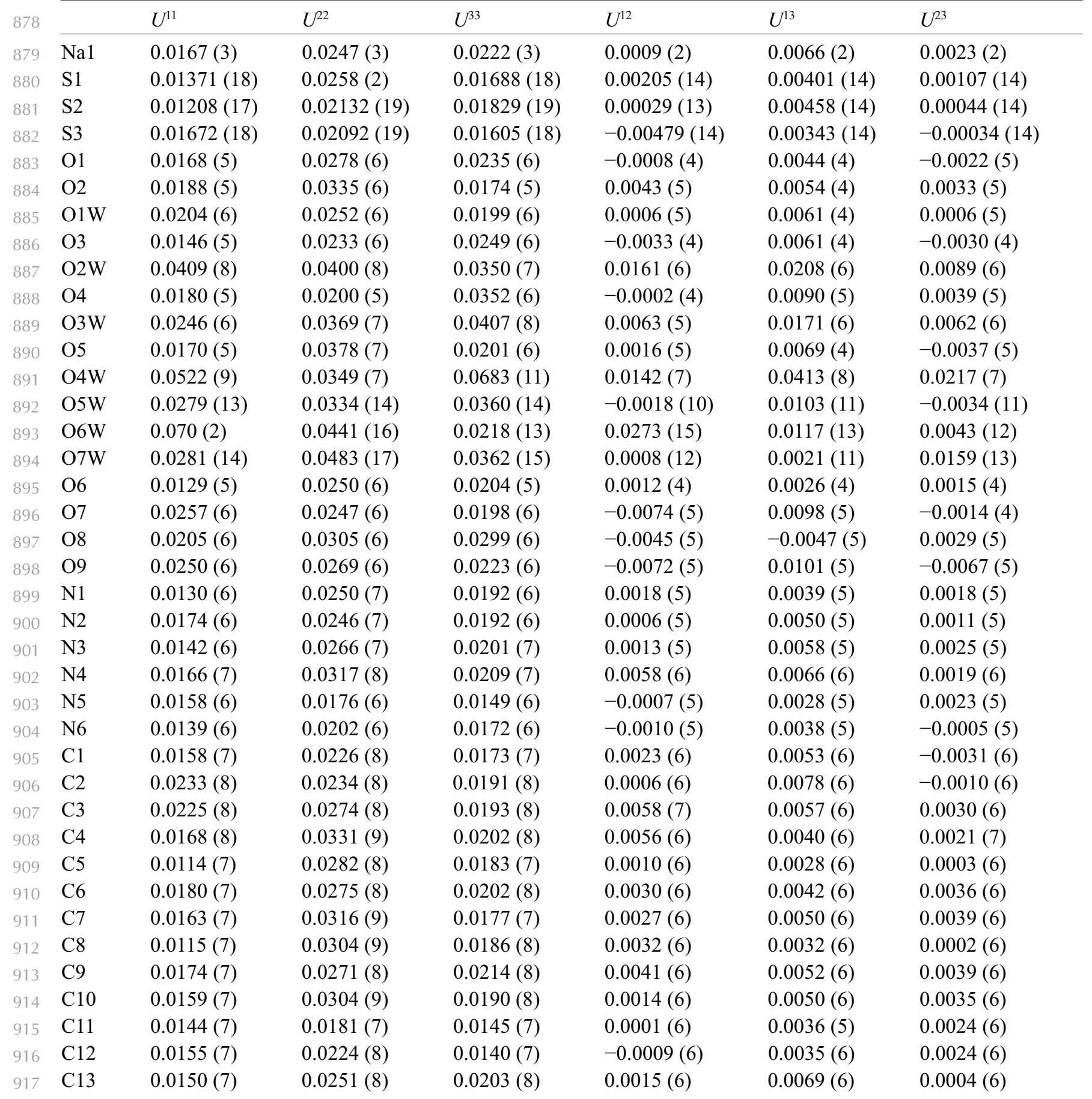




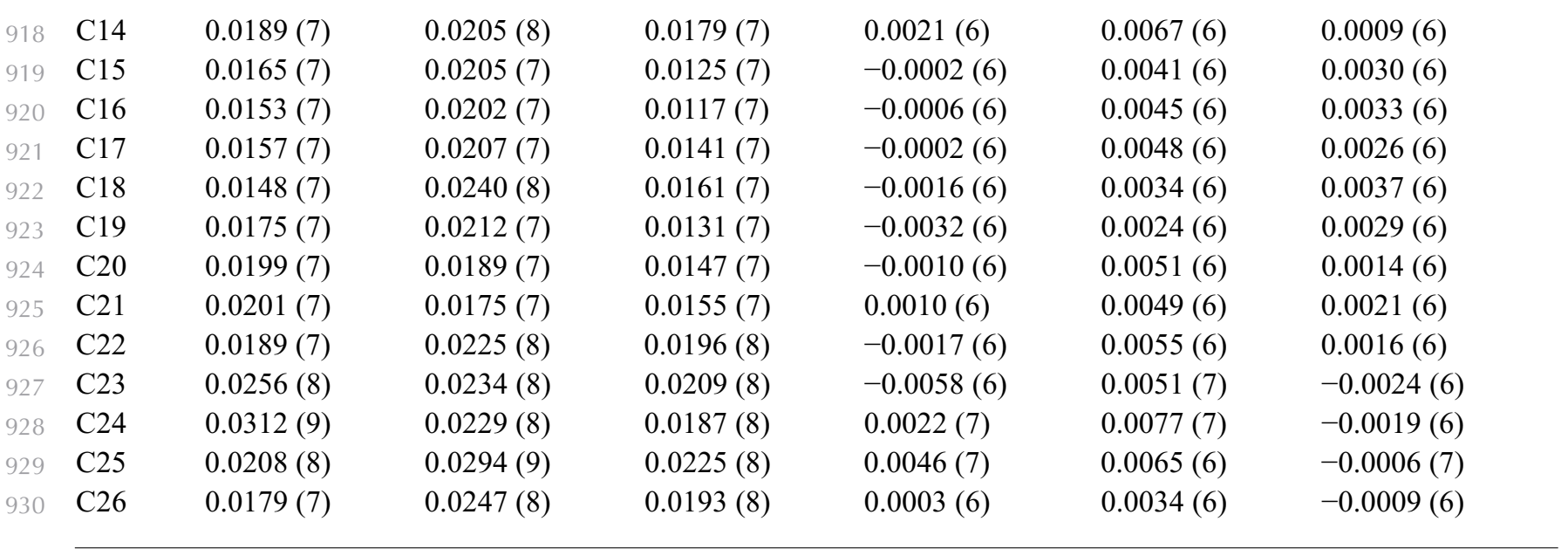

Geometric parameters $(\AA, \stackrel{\circ}{)})$

\begin{tabular}{|c|c|c|c|}
\hline $\mathrm{Na} 1-\mathrm{O} 1 \mathrm{~W}$ & $2.3184(13)$ & $\mathrm{N} 5-\mathrm{C} 11$ & $1.3322(19)$ \\
\hline $\mathrm{Na} 1-\mathrm{O} 4 \mathrm{~W}$ & $2.3211(15)$ & $\mathrm{N} 6-\mathrm{C} 21$ & $1.409(2)$ \\
\hline $\mathrm{Na} 1-\mathrm{O} 3$ & $2.4042(12)$ & $\mathrm{N} 6-\mathrm{H} 1 \mathrm{~N}$ & $0.92(2)$ \\
\hline $\mathrm{Na} 1-\mathrm{O}^{\mathrm{i}}$ & $2.4237(12)$ & $\mathrm{C} 2-\mathrm{C} 3$ & $1.393(2)$ \\
\hline $\mathrm{Na} 1-\mathrm{O} 3 \mathrm{~W}$ & $2.4592(14)$ & $\mathrm{C} 2-\mathrm{H} 2$ & 0.9500 \\
\hline $\mathrm{Na} 1-\mathrm{O} 2 \mathrm{~W}$ & $2.4764(14)$ & $\mathrm{C} 3-\mathrm{C} 4$ & $1.364(2)$ \\
\hline $\mathrm{S} 1-\mathrm{O} 2$ & $1.4428(11)$ & $\mathrm{C} 3-\mathrm{H} 3$ & 0.9500 \\
\hline $\mathrm{S} 1-\mathrm{O} 1$ & $1.4433(12)$ & $\mathrm{C} 4-\mathrm{H} 4$ & 0.9500 \\
\hline $\mathrm{S} 1-\mathrm{N} 1$ & $1.6055(13)$ & $\mathrm{C} 5-\mathrm{C} 10$ & $1.388(2)$ \\
\hline $\mathrm{S} 1-\mathrm{C} 5$ & $1.7674(16)$ & $\mathrm{C} 5-\mathrm{C} 6$ & $1.395(2)$ \\
\hline $\mathrm{S} 2-\mathrm{O} 4$ & $1.4419(11)$ & $\mathrm{C} 6-\mathrm{C} 7$ & $1.386(2)$ \\
\hline $\mathrm{S} 2-\mathrm{O} 5$ & $1.4554(12)$ & C6- $\mathrm{H} 6$ & 0.9500 \\
\hline $\mathrm{S} 2-\mathrm{O} 6$ & $1.4735(11)$ & $\mathrm{C} 7-\mathrm{C} 8$ & $1.388(2)$ \\
\hline $\mathrm{S} 2-\mathrm{C} 17$ & $1.8048(15)$ & $\mathrm{C} 7-\mathrm{H} 7$ & 0.9500 \\
\hline $\mathrm{S} 3-\mathrm{O} 8$ & $1.4506(12)$ & $\mathrm{C} 8-\mathrm{C} 9$ & $1.382(2)$ \\
\hline $\mathrm{S} 3-\mathrm{O} 7$ & $1.4516(11)$ & $\mathrm{C} 9-\mathrm{C} 10$ & $1.385(2)$ \\
\hline S3-O9 & $1.4605(12)$ & C9- $\mathrm{H} 9$ & 0.9500 \\
\hline $\mathrm{S} 3-\mathrm{C} 19$ & $1.7769(15)$ & $\mathrm{C} 10-\mathrm{H} 10$ & 0.9500 \\
\hline $\mathrm{O} 1 \mathrm{~W}-\mathrm{H} 1 \mathrm{~W}$ & $0.88(3)$ & $\mathrm{C} 11-\mathrm{C} 16$ & $1.463(2)$ \\
\hline $\mathrm{O} 1 \mathrm{~W}-\mathrm{H} 2 \mathrm{~W}$ & $0.91(3)$ & $\mathrm{C} 11-\mathrm{C} 12$ & $1.467(2)$ \\
\hline $\mathrm{O} 3-\mathrm{C} 12$ & $1.2626(18)$ & $\mathrm{C} 12-\mathrm{C} 13$ & $1.441(2)$ \\
\hline $\mathrm{O} 2 \mathrm{~W}-\mathrm{H} 3 \mathrm{~W}$ & $0.86(3)$ & $\mathrm{C} 13-\mathrm{C} 14$ & $1.343(2)$ \\
\hline $\mathrm{O} 2 \mathrm{~W}-\mathrm{H} 4 \mathrm{~W}$ & $0.92(4)$ & $\mathrm{C} 13-\mathrm{H} 13$ & 0.9500 \\
\hline $\mathrm{O} 3 \mathrm{~W}-\mathrm{H} 5 \mathrm{~W}$ & $0.86(3)$ & $\mathrm{C} 14-\mathrm{C} 15$ & $1.448(2)$ \\
\hline $\mathrm{O} 3 \mathrm{~W}-\mathrm{H} 6 \mathrm{~W}$ & $0.83(4)$ & $\mathrm{C} 14-\mathrm{H} 14$ & 0.9500 \\
\hline $\mathrm{O} 4 \mathrm{~W}-\mathrm{H} 7 \mathrm{~W}$ & $0.879(10)$ & $\mathrm{C} 15-\mathrm{C} 20$ & $1.401(2)$ \\
\hline $\mathrm{O} 4 \mathrm{~W}-\mathrm{H} 8 \mathrm{~W}$ & $0.883(10)$ & $\mathrm{C} 15-\mathrm{C} 16$ & $1.421(2)$ \\
\hline $\mathrm{O} 4 \mathrm{~W}-\mathrm{H} 14 \mathrm{~W}$ & $0.886(10)$ & $\mathrm{C} 16-\mathrm{C} 17$ & $1.427(2)$ \\
\hline $\mathrm{O} 5 \mathrm{~W}-\mathrm{H} 9 \mathrm{~W}$ & $0.883(10)$ & $\mathrm{C} 17-\mathrm{C} 18$ & $1.386(2)$ \\
\hline $\mathrm{O} 5 \mathrm{~W}-\mathrm{H} 10 \mathrm{~W}$ & $0.885(10)$ & $\mathrm{C} 18-\mathrm{C} 19$ & $1.393(2)$ \\
\hline $\mathrm{O} 6 \mathrm{~W}-\mathrm{H} 11 \mathrm{~W}$ & $0.886(10)$ & $\mathrm{C} 18-\mathrm{H} 18$ & 0.9500 \\
\hline $\mathrm{O} 6 \mathrm{~W}-\mathrm{H} 12 \mathrm{~W}$ & $0.881(10)$ & $\mathrm{C} 19-\mathrm{C} 20$ & $1.377(2)$ \\
\hline
\end{tabular}




\begin{tabular}{|c|c|}
\hline 964 & $\mathrm{O} 7 \mathrm{~W}-\mathrm{H} 13 \mathrm{~W}$ \\
\hline 965 & $\mathrm{O} 7-\mathrm{Na} 1^{\mathrm{i}}$ \\
\hline 966 & $\mathrm{~N} 1-\mathrm{C} 1$ \\
\hline 967 & $\mathrm{~N} 2-\mathrm{C} 2$ \\
\hline 968 & $\mathrm{~N} 2-\mathrm{C} 1$ \\
\hline 969 & $\mathrm{~N} 3-\mathrm{C} 4$ \\
\hline 970 & $\mathrm{~N} 3-\mathrm{C} 1$ \\
\hline 971 & $\mathrm{~N} 3-\mathrm{H} 5 \mathrm{~N}$ \\
\hline 972 & $\mathrm{~N} 4-\mathrm{C} 8$ \\
\hline 973 & $\mathrm{~N} 4-\mathrm{H} 2 \mathrm{~N}$ \\
\hline 974 & $\mathrm{~N} 4-\mathrm{H} 3 \mathrm{~N}$ \\
\hline 975 & $\mathrm{~N} 4-\mathrm{H} 4 \mathrm{~N}$ \\
\hline 976 & N5-N6 \\
\hline 977 & \\
\hline 978 & $\mathrm{O} 1 \mathrm{~W}-\mathrm{Na} 1-\mathrm{O} 4 \mathrm{~W}$ \\
\hline 979 & $\mathrm{O} 1 \mathrm{~W}-\mathrm{Na} 1-\mathrm{O} 3$ \\
\hline 980 & $\mathrm{O} 4 \mathrm{~W}-\mathrm{Na} 1-\mathrm{O} 3$ \\
\hline 981 & $\mathrm{O} 1 \mathrm{~W}-\mathrm{Na} 1-\mathrm{O}^{\mathrm{i}}$ \\
\hline 982 & $\mathrm{O} 4 \mathrm{~W}-\mathrm{Na} 1-\mathrm{O} 7^{\mathrm{i}}$ \\
\hline 983 & $\mathrm{O} 3-\mathrm{Na} 1-\mathrm{O}^{\mathrm{i}}$ \\
\hline 984 & $\mathrm{O} 1 \mathrm{~W}-\mathrm{Na} 1-\mathrm{O} 3 \mathrm{~W}$ \\
\hline 985 & $\mathrm{O} 4 \mathrm{~W}-\mathrm{Na} 1-\mathrm{O} 3 \mathrm{~W}$ \\
\hline 986 & $\mathrm{O} 3-\mathrm{Na} 1-\mathrm{O} 3 \mathrm{~W}$ \\
\hline 987 & $\mathrm{O} 7 \mathrm{i}-\mathrm{Na} 1-\mathrm{O} 3 \mathrm{~W}$ \\
\hline 988 & $\mathrm{O} 1 \mathrm{~W}-\mathrm{Na} 1-\mathrm{O} 2 \mathrm{~W}$ \\
\hline 989 & $\mathrm{O} 4 \mathrm{~W}-\mathrm{Na} 1-\mathrm{O} 2 \mathrm{~W}$ \\
\hline 990 & $\mathrm{O} 3-\mathrm{Na} 1-\mathrm{O} 2 \mathrm{~W}$ \\
\hline 991 & $\mathrm{O} 7^{\mathrm{i}}-\mathrm{Na} 1-\mathrm{O} 2 \mathrm{~W}$ \\
\hline 992 & $\mathrm{O} 3 \mathrm{~W}-\mathrm{Na} 1-\mathrm{O} 2 \mathrm{~W}$ \\
\hline 993 & $\mathrm{O} 2-\mathrm{S} 1-\mathrm{O} 1$ \\
\hline 994 & $\mathrm{O} 2-\mathrm{S} 1-\mathrm{N} 1$ \\
\hline 995 & $\mathrm{O} 1-\mathrm{S} 1-\mathrm{N} 1$ \\
\hline 996 & $\mathrm{O} 2-\mathrm{S} 1-\mathrm{C} 5$ \\
\hline 997 & $\mathrm{O} 1-\mathrm{S} 1-\mathrm{C} 5$ \\
\hline 998 & $\mathrm{~N} 1-\mathrm{S} 1-\mathrm{C} 5$ \\
\hline 999 & $\mathrm{O} 4-\mathrm{S} 2-\mathrm{O} 5$ \\
\hline 1000 & $\mathrm{O} 4-\mathrm{S} 2-\mathrm{O} 6$ \\
\hline 1001 & $\mathrm{O} 5-\mathrm{S} 2-\mathrm{O} 6$ \\
\hline 1002 & $\mathrm{O} 4-\mathrm{S} 2-\mathrm{C} 17$ \\
\hline 1003 & $\mathrm{O} 5-\mathrm{S} 2-\mathrm{C} 17$ \\
\hline 1004 & $\mathrm{O} 6-\mathrm{S} 2-\mathrm{C} 17$ \\
\hline 1005 & $\mathrm{O} 8-\mathrm{S} 3-\mathrm{O} 7$ \\
\hline 1006 & $\mathrm{O} 8-\mathrm{S} 3-\mathrm{O} 9$ \\
\hline 1007 & $\mathrm{O} 7-\mathrm{S} 3-\mathrm{O} 9$ \\
\hline 1008 & $\mathrm{O} 8-\mathrm{S} 3-\mathrm{C} 19$ \\
\hline 1009 & $\mathrm{O} 7-\mathrm{S} 3-\mathrm{C} 19$ \\
\hline 1010 & $\mathrm{O} 9-\mathrm{S} 3-\mathrm{C} 19$ \\
\hline
\end{tabular}

$0.875(10)$

2.4237 (12)

$1.337(2)$

$1.333(2)$

$1.345(2)$

$1.344(2)$

$1.369(2)$

$0.84(2)$

$1.465(2)$

$0.92(3)$

$0.91(2)$

0.91 (2)

1.3031 (18)

$169.11(5)$

$90.96(4)$

$98.12(5)$

$85.13(4)$

$101.30(5)$

$86.81(4)$

$87.73(5)$

$84.75(6)$

$166.97(5)$

$80.17(5)$

$81.14(5)$

$91.10(6)$

$101.64(5)$

$163.94(5)$

$90.97(5)$

$116.71(7)$

$104.29(7)$

$113.65(7)$

$106.55(7)$

$108.68(7)$

$106.26(7)$

$115.51(7)$

$111.69(7)$

$110.45(6)$

$108.60(7)$

$105.90(7)$

103.85 (7)

113.59 (7)

$112.90(7)$

$112.34(7)$

$105.75(7)$

$105.76(7)$

105.67 (7)

111.8 (16)
C20- $\mathrm{H} 20$

$\mathrm{C} 21-\mathrm{C} 22$

$\mathrm{C} 21-\mathrm{C} 26$

$\mathrm{C} 22-\mathrm{C} 23$

$\mathrm{C} 22-\mathrm{H} 22$

$\mathrm{C} 23-\mathrm{C} 24$

$\mathrm{C} 23-\mathrm{H} 23$

$\mathrm{C} 24-\mathrm{C} 25$

C24- 24

C25-C26

$\mathrm{C} 25-\mathrm{H} 25$

C26-H26

$\mathrm{C} 4-\mathrm{C} 3-\mathrm{C} 2$

$\mathrm{C} 4-\mathrm{C} 3-\mathrm{H} 3$

$\mathrm{C} 2-\mathrm{C} 3-\mathrm{H} 3$

$\mathrm{N} 3-\mathrm{C} 4-\mathrm{C} 3$

$\mathrm{N} 3-\mathrm{C} 4-\mathrm{H} 4$

$\mathrm{C} 3-\mathrm{C} 4-\mathrm{H} 4$

$\mathrm{C} 10-\mathrm{C} 5-\mathrm{C} 6$

$\mathrm{C} 10-\mathrm{C} 5-\mathrm{S} 1$

C6- C5-S1

$\mathrm{C} 7-\mathrm{C} 6-\mathrm{C} 5$

C7- 6 - $-\mathrm{H} 6$

$\mathrm{C} 5-\mathrm{C} 6-\mathrm{H} 6$

C6- C7-C8

$\mathrm{C} 6-\mathrm{C} 7-\mathrm{H} 7$

$\mathrm{C} 8-\mathrm{C} 7-\mathrm{H} 7$

$\mathrm{C} 9-\mathrm{C} 8-\mathrm{C} 7$

C9- 8 - $-\mathrm{N} 4$

$\mathrm{C} 7-\mathrm{C} 8-\mathrm{N} 4$

$\mathrm{C} 8-\mathrm{C} 9-\mathrm{C} 10$

C8- $99-\mathrm{H} 9$

$\mathrm{C} 10-\mathrm{C} 9-\mathrm{H} 9$

C9- $10-\mathrm{C} 5$

C9- $\mathrm{C} 10-\mathrm{H} 10$

$\mathrm{C} 5-\mathrm{C} 10-\mathrm{H} 10$

N5-C11-C16

N5-C11-C12

$\mathrm{C} 16-\mathrm{C} 11-\mathrm{C} 12$

$\mathrm{O} 3-\mathrm{C} 12-\mathrm{C} 13$

$\mathrm{O} 3-\mathrm{C} 12-\mathrm{C} 11$

$\mathrm{C} 13-\mathrm{C} 12-\mathrm{C} 11$

$\mathrm{C} 14-\mathrm{C} 13-\mathrm{C} 12$

C14-C13-H13

C12-C13-H13

$\mathrm{C} 13-\mathrm{C} 14-\mathrm{C} 15$
0.9500

1.394 (2)

$1.395(2)$

$1.387(2)$

0.9500

$1.388(2)$

0.9500

$1.393(2)$

0.9500

1.388 (2)

0.9500

0.9500

$116.51(15)$

121.7

121.7

$119.48(15)$

120.3

120.3

$121.41(15)$

$117.89(12)$

$120.71(12)$

118.90 (15)

120.6

120.6

119.27 (15)

120.4

120.4

$121.88(15)$

$118.81(15)$

$119.30(14)$

$119.06(15)$

120.5

120.5

119.43 (15)

120.3

120.3

$116.76(13)$

$122.51(13)$

120.25 (13)

120.68 (13)

121.14 (14)

118.06 (13)

120.66 (14)

119.7

119.7

122.71 (14) 
$\mathrm{Na} 1-\mathrm{O} 1 \mathrm{~W}-\mathrm{H} 2 \mathrm{~W}$

$\mathrm{H} 1 \mathrm{~W}-\mathrm{O} 1 \mathrm{~W}-\mathrm{H} 2 \mathrm{~W}$

$\mathrm{C} 12-\mathrm{O} 3-\mathrm{Na} 1$

$\mathrm{Na} 1-\mathrm{O} 2 \mathrm{~W}-\mathrm{H} 3 \mathrm{~W}$

$\mathrm{Na} 1-\mathrm{O} 2 \mathrm{~W}-\mathrm{H} 4 \mathrm{~W}$

$\mathrm{H} 3 \mathrm{~W}-\mathrm{O} 2 \mathrm{~W}-\mathrm{H} 4 \mathrm{~W}$

$\mathrm{Na} 1-\mathrm{O} 3 \mathrm{~W}-\mathrm{H} 5 \mathrm{~W}$

$\mathrm{Na} 1-\mathrm{O} 3 \mathrm{~W}-\mathrm{H} 6 \mathrm{~W}$

$\mathrm{H} 5 \mathrm{~W}-\mathrm{O} 3 \mathrm{~W}-\mathrm{H} 6 \mathrm{~W}$

$\mathrm{Na} 1-\mathrm{O} 4 \mathrm{~W}-\mathrm{H} 7 \mathrm{~W}$

$\mathrm{Na} 1-\mathrm{O} 4 \mathrm{~W}-\mathrm{H} 8 \mathrm{~W}$

$\mathrm{H} 7 \mathrm{~W}-\mathrm{O} 4 \mathrm{~W}-\mathrm{H} 8 \mathrm{~W}$

$\mathrm{Na} 1-\mathrm{O} 4 \mathrm{~W}-\mathrm{H} 14 \mathrm{~W}$

$\mathrm{H} 7 \mathrm{~W}-\mathrm{O} 4 \mathrm{~W}-\mathrm{H} 14 \mathrm{~W}$

$\mathrm{H} 9 \mathrm{~W}-\mathrm{O} 5 \mathrm{~W}-\mathrm{H} 10 \mathrm{~W}$

$\mathrm{H} 11 \mathrm{~W}-\mathrm{O} 6 \mathrm{~W}-\mathrm{H} 12 \mathrm{~W}$

$\mathrm{S} 3-\mathrm{O} 7-\mathrm{Na} 1^{\mathrm{i}}$

$\mathrm{C} 1-\mathrm{N} 1-\mathrm{S} 1$

$\mathrm{C} 2-\mathrm{N} 2-\mathrm{C} 1$

$\mathrm{C} 4-\mathrm{N} 3-\mathrm{C} 1$

$\mathrm{C} 4-\mathrm{N} 3-\mathrm{H} 5 \mathrm{~N}$

$\mathrm{C} 1-\mathrm{N} 3-\mathrm{H} 5 \mathrm{~N}$

$\mathrm{C} 8-\mathrm{N} 4-\mathrm{H} 2 \mathrm{~N}$

$\mathrm{C} 8-\mathrm{N} 4-\mathrm{H} 3 \mathrm{~N}$

$\mathrm{H} 2 \mathrm{~N}-\mathrm{N} 4-\mathrm{H} 3 \mathrm{~N}$

$\mathrm{C} 8-\mathrm{N} 4-\mathrm{H} 4 \mathrm{~N}$

$\mathrm{H} 2 \mathrm{~N}-\mathrm{N} 4-\mathrm{H} 4 \mathrm{~N}$

$\mathrm{H} 3 \mathrm{~N}-\mathrm{N} 4-\mathrm{H} 4 \mathrm{~N}$

$\mathrm{N} 6-\mathrm{N} 5-\mathrm{C} 11$

$\mathrm{N} 5-\mathrm{N} 6-\mathrm{C} 21$

$\mathrm{N} 5-\mathrm{N} 6-\mathrm{H} 1 \mathrm{~N}$

$\mathrm{C} 21-\mathrm{N} 6-\mathrm{H} 1 \mathrm{~N}$

$\mathrm{N} 1-\mathrm{C} 1-\mathrm{N} 2$

$\mathrm{N} 1-\mathrm{C} 1-\mathrm{N} 3$

$\mathrm{N} 2-\mathrm{C} 1-\mathrm{N} 3$

$\mathrm{N} 2-\mathrm{C} 2-\mathrm{C} 3$

$\mathrm{N} 2-\mathrm{C} 2-\mathrm{H} 2$

$\mathrm{C} 3-\mathrm{C} 2-\mathrm{H} 2$

$\mathrm{O} 8-\mathrm{S} 3-\mathrm{O} 7-\mathrm{Na} 1^{\mathrm{i}}$

$\mathrm{O} 9-\mathrm{S} 3-\mathrm{O} 7-\mathrm{Na} 1^{\mathrm{i}}$

$\mathrm{C} 19-\mathrm{S} 3-\mathrm{O} 7-\mathrm{Na} 1^{\mathrm{i}}$

$\mathrm{O} 2-\mathrm{S} 1-\mathrm{N} 1-\mathrm{C} 1$

$\mathrm{O} 1-\mathrm{S} 1-\mathrm{N} 1-\mathrm{C} 1$

$\mathrm{C} 5-\mathrm{S} 1-\mathrm{N} 1-\mathrm{C} 1$

$\mathrm{C} 11-\mathrm{N} 5-\mathrm{N} 6-\mathrm{C} 21$

$\mathrm{S} 1-\mathrm{N} 1-\mathrm{C} 1-\mathrm{N} 2$

$\mathrm{S} 1-\mathrm{N} 1-\mathrm{C} 1-\mathrm{N} 3$
121.3 (16)

103 (2)

$136.93(10)$

110.0 (18)

124 (2)

$102(3)$

119.2 (19)

$115(2)$

$100(3)$

$137.7(16)$

108 (3)

98 (2)

$118(3)$

99 (2)

99 (2)

$96(2)$

138.42 (7)

$123.67(11)$

$117.89(13)$

$122.12(14)$

$121.4(15)$

$116.4(15)$

$109.0(15)$

$109.8(14)$

111 (2)

$109.3(14)$

111 (2)

106.8 (19)

$118.99(13)$

$121.27(13)$

115.4 (14)

123.2 (14)

$125.95(14)$

$114.28(14)$

119.77 (14)

$124.12(15)$

117.9

117.9

81.07 (11)

$-149.26(9)$

$-34.46(12)$

$156.92(13)$

$28.75(15)$

$-90.72(14)$

$174.83(13)$

$8.8(2)$

-170.92 (11)
C13-C14-H14

$\mathrm{C} 15-\mathrm{C} 14-\mathrm{H} 14$

$\mathrm{C} 20-\mathrm{C} 15-\mathrm{C} 16$

$\mathrm{C} 20-\mathrm{C} 15-\mathrm{C} 14$

$\mathrm{C} 16-\mathrm{C} 15-\mathrm{C} 14$

$\mathrm{C} 15-\mathrm{C} 16-\mathrm{C} 17$

$\mathrm{C} 15-\mathrm{C} 16-\mathrm{C} 11$

C17-C16-C11

C18-C17-C16

$\mathrm{C} 18-\mathrm{C} 17-\mathrm{S} 2$

$\mathrm{C} 16-\mathrm{C} 17-\mathrm{S} 2$

C17-C18-C19

C17-C18-H18

C19-C18-H18

$\mathrm{C} 20-\mathrm{C} 19-\mathrm{C} 18$

$\mathrm{C} 20-\mathrm{C} 19-\mathrm{S} 3$

$\mathrm{C} 18-\mathrm{C} 19-\mathrm{S} 3$

C19-C20-C15

C19-C20-H20

$\mathrm{C} 15-\mathrm{C} 20-\mathrm{H} 20$

$\mathrm{C} 22-\mathrm{C} 21-\mathrm{C} 26$

$\mathrm{C} 22-\mathrm{C} 21-\mathrm{N} 6$

$\mathrm{C} 26-\mathrm{C} 21-\mathrm{N} 6$

$\mathrm{C} 23-\mathrm{C} 22-\mathrm{C} 21$

$\mathrm{C} 23-\mathrm{C} 22-\mathrm{H} 22$

$\mathrm{C} 21-\mathrm{C} 22-\mathrm{H} 22$

$\mathrm{C} 22-\mathrm{C} 23-\mathrm{C} 24$

$\mathrm{C} 22-\mathrm{C} 23-\mathrm{H} 23$

$\mathrm{C} 24-\mathrm{C} 23-\mathrm{H} 23$

$\mathrm{C} 23-\mathrm{C} 24-\mathrm{C} 25$

$\mathrm{C} 23-\mathrm{C} 24-\mathrm{H} 24$

$\mathrm{C} 25-\mathrm{C} 24-\mathrm{H} 24$

$\mathrm{C} 26-\mathrm{C} 25-\mathrm{C} 24$

$\mathrm{C} 26-\mathrm{C} 25-\mathrm{H} 25$

$\mathrm{C} 24-\mathrm{C} 25-\mathrm{H} 25$

$\mathrm{C} 25-\mathrm{C} 26-\mathrm{C} 21$

$\mathrm{C} 25-\mathrm{C} 26-\mathrm{H} 26$

$\mathrm{C} 21-\mathrm{C} 26-\mathrm{H} 26$

$\mathrm{C} 13-\mathrm{C} 14-\mathrm{C} 15-\mathrm{C} 20$

$\mathrm{C} 13-\mathrm{C} 14-\mathrm{C} 15-\mathrm{C} 16$

$\mathrm{C} 20-\mathrm{C} 15-\mathrm{C} 16-\mathrm{C} 17$

$\mathrm{C} 14-\mathrm{C} 15-\mathrm{C} 16-\mathrm{C} 17$

$\mathrm{C} 20-\mathrm{C} 15-\mathrm{C} 16-\mathrm{C} 11$

$\mathrm{C} 14-\mathrm{C} 15-\mathrm{C} 16-\mathrm{C} 11$

N5-C11-C16-C15

$\mathrm{C} 12-\mathrm{C} 11-\mathrm{C} 16-\mathrm{C} 15$

N5-C11-C16-C17
118.6

118.6

$121.48(13)$

118.39 (14)

$120.13(13)$

$116.75(13)$

$117.08(13)$

$126.18(13)$

$120.02(14)$

$113.36(11)$

$126.58(11)$

$121.74(14)$

119.1

119.1

119.40 (14)

$121.46(12)$

119.13 (11)

120.10 (14)

119.9

119.9

121.42 (14)

$116.56(13)$

$122.01(14)$

119.28 (15)

120.4

120.4

120.15 (15)

119.9

119.9

119.87 (15)

120.1

120.1

$121.02(15)$

119.5

119.5

118.25 (14)

120.9

120.9

$-178.66(14)$

$1.2(2)$

6.9 (2)

$-172.92(13)$

$-172.79(13)$

7.4 (2)

$159.68(13)$

$-12.5(2)$

-20.0 (2) 


\begin{tabular}{|c|c|c|}
\hline 1060 & $\mathrm{C} 2-\mathrm{N} 2-\mathrm{C} 1-\mathrm{N} 1$ & $-176.66(15)$ \\
\hline 1061 & $\mathrm{C} 2-\mathrm{N} 2-\mathrm{C} 1-\mathrm{N} 3$ & $3.0(2)$ \\
\hline 1062 & $\mathrm{C} 4-\mathrm{N} 3-\mathrm{C} 1-\mathrm{N} 1$ & $175.88(14)$ \\
\hline 1063 & $\mathrm{C} 4-\mathrm{N} 3-\mathrm{C} 1-\mathrm{N} 2$ & $-3.8(2)$ \\
\hline 1064 & $\mathrm{C} 1-\mathrm{N} 2-\mathrm{C} 2-\mathrm{C} 3$ & $-0.2(2)$ \\
\hline 65 & $\mathrm{~N} 2-\mathrm{C} 2-\mathrm{C} 3-\mathrm{C} 4$ & $-1.9(2)$ \\
\hline 66 & $\mathrm{C} 1-\mathrm{N} 3-\mathrm{C} 4-\mathrm{C} 3$ & $1.6(2)$ \\
\hline 67 & $\mathrm{C} 2-\mathrm{C} 3-\mathrm{C} 4-\mathrm{N} 3$ & $1.1(2)$ \\
\hline 68 & $\mathrm{O} 2-\mathrm{S} 1-\mathrm{C} 5-\mathrm{C} 10$ & $9.38(14)$ \\
\hline 69 & $\mathrm{O} 1-\mathrm{S} 1-\mathrm{C} 5-\mathrm{C} 10$ & $135.92(12)$ \\
\hline 970 & $\mathrm{~N} 1-\mathrm{S} 1-\mathrm{C} 5-\mathrm{C} 10$ & $-101.41(13)$ \\
\hline D71 & $\mathrm{O} 2-\mathrm{S} 1-\mathrm{C} 5-\mathrm{C} 6$ & $-171.14(12)$ \\
\hline 772 & $\mathrm{O} 1-\mathrm{S} 1-\mathrm{C} 5-\mathrm{C} 6$ & $-44.60(14)$ \\
\hline 73 & $\mathrm{~N} 1-\mathrm{S} 1-\mathrm{C} 5-\mathrm{C} 6$ & $78.07(14)$ \\
\hline 74 & $\mathrm{C} 10-\mathrm{C} 5-\mathrm{C} 6-\mathrm{C} 7$ & $1.3(2)$ \\
\hline 1075 & $\mathrm{~S} 1-\mathrm{C} 5-\mathrm{C} 6-\mathrm{C} 7$ & $-178.11(12)$ \\
\hline 976 & $\mathrm{C} 5-\mathrm{C} 6-\mathrm{C} 7-\mathrm{C} 8$ & $0.2(2)$ \\
\hline 77 & $\mathrm{C} 6-\mathrm{C} 7-\mathrm{C} 8-\mathrm{C} 9$ & $-2.2(2)$ \\
\hline 1078 & $\mathrm{C} 6-\mathrm{C} 7-\mathrm{C} 8-\mathrm{N} 4$ & $178.48(14)$ \\
\hline 1079 & $\mathrm{C} 7-\mathrm{C} 8-\mathrm{C} 9-\mathrm{C} 10$ & $2.5(2)$ \\
\hline 1080 & $\mathrm{~N} 4-\mathrm{C} 8-\mathrm{C} 9-\mathrm{C} 10$ & $-178.13(14)$ \\
\hline 81 & $\mathrm{C} 8-\mathrm{C} 9-\mathrm{C} 10-\mathrm{C} 5$ & $-0.9(2)$ \\
\hline 1082 & $\mathrm{C} 6-\mathrm{C} 5-\mathrm{C} 10-\mathrm{C} 9$ & $-1.0(2)$ \\
\hline 1083 & $\mathrm{~S} 1-\mathrm{C} 5-\mathrm{C} 10-\mathrm{C} 9$ & $178.47(12)$ \\
\hline 1084 & $\mathrm{~N} 6-\mathrm{N} 5-\mathrm{C} 11-\mathrm{C} 16$ & $-178.41(12)$ \\
\hline 1085 & $\mathrm{~N} 6-\mathrm{N} 5-\mathrm{C} 11-\mathrm{C} 12$ & $-6.4(2)$ \\
\hline 1086 & $\mathrm{Na} 1-\mathrm{O} 3-\mathrm{C} 12-\mathrm{C} 13$ & $6.3(2)$ \\
\hline & $\mathrm{Na} 1-\mathrm{O} 3-\mathrm{C} 12-\mathrm{C} 11$ & $-169.80(10)$ \\
\hline 1088 & $\mathrm{~N} 5-\mathrm{C} 11-\mathrm{C} 12-\mathrm{O} 3$ & $13.8(2)$ \\
\hline & $\mathrm{C} 16-\mathrm{C} 11-\mathrm{C} 12-\mathrm{O} 3$ & $-174.40(13)$ \\
\hline & $\mathrm{N} 5-\mathrm{C} 11-\mathrm{C} 12-\mathrm{C} 13$ & $-162.36(14)$ \\
\hline & $\mathrm{C} 16-\mathrm{C} 11-\mathrm{C} 12-\mathrm{C} 13$ & $9.4(2)$ \\
\hline & $\mathrm{O} 3-\mathrm{C} 12-\mathrm{C} 13-\mathrm{C} 14$ & $-176.90(14)$ \\
\hline & $\mathrm{C} 11-\mathrm{C} 12-\mathrm{C} 13-\mathrm{C} 14$ & $-0.7(2)$ \\
\hline & $\mathrm{C} 12-\mathrm{C} 13-\mathrm{C} 14-\mathrm{C} 15$ & $-4.7(2)$ \\
\hline
\end{tabular}

\begin{tabular}{|c|c|}
\hline $\mathrm{C} 12-\mathrm{C} 11-\mathrm{C} 16-\mathrm{C} 17$ & $167.76(14)$ \\
\hline $\mathrm{C} 15-\mathrm{C} 16-\mathrm{C} 17-\mathrm{C} 18$ & $-7.1(2)$ \\
\hline $\mathrm{C} 11-\mathrm{C} 16-\mathrm{C} 17-\mathrm{C} 18$ & $172.62(14)$ \\
\hline $\mathrm{C} 15-\mathrm{C} 16-\mathrm{C} 17-\mathrm{S} 2$ & $170.58(11)$ \\
\hline $\mathrm{C} 11-\mathrm{C} 16-\mathrm{C} 17-\mathrm{S} 2$ & $-9.7(2)$ \\
\hline $\mathrm{O} 4-\mathrm{S} 2-\mathrm{C} 17-\mathrm{C} 18$ & $141.14(11)$ \\
\hline $\mathrm{O} 5-\mathrm{S} 2-\mathrm{C} 17-\mathrm{C} 18$ & $-94.23(12)$ \\
\hline $\mathrm{O} 6-\mathrm{S} 2-\mathrm{C} 17-\mathrm{C} 18$ & $22.15(13)$ \\
\hline $\mathrm{O} 4-\mathrm{S} 2-\mathrm{C} 17-\mathrm{C} 16$ & $-36.66(15)$ \\
\hline $\mathrm{O} 5-\mathrm{S} 2-\mathrm{C} 17-\mathrm{C} 16$ & $87.97(14)$ \\
\hline $\mathrm{O} 6-\mathrm{S} 2-\mathrm{C} 17-\mathrm{C} 16$ & $-155.65(13)$ \\
\hline $\mathrm{C} 16-\mathrm{C} 17-\mathrm{C} 18-\mathrm{C} 19$ & $1.8(2)$ \\
\hline $\mathrm{S} 2-\mathrm{C} 17-\mathrm{C} 18-\mathrm{C} 19$ & $-176.12(11)$ \\
\hline $\mathrm{C} 17-\mathrm{C} 18-\mathrm{C} 19-\mathrm{C} 20$ & $4.0(2)$ \\
\hline $\mathrm{C} 17-\mathrm{C} 18-\mathrm{C} 19-\mathrm{S} 3$ & $-174.87(11)$ \\
\hline $\mathrm{O} 8-\mathrm{S} 3-\mathrm{C} 19-\mathrm{C} 20$ & $143.56(13)$ \\
\hline $\mathrm{O} 7-\mathrm{S} 3-\mathrm{C} 19-\mathrm{C} 20$ & $-95.67(13)$ \\
\hline $\mathrm{O} 9-\mathrm{S} 3-\mathrm{C} 19-\mathrm{C} 20$ & $23.63(14)$ \\
\hline $\mathrm{O} 8-\mathrm{S} 3-\mathrm{C} 19-\mathrm{C} 18$ & $-37.62(14)$ \\
\hline $\mathrm{O} 7-\mathrm{S} 3-\mathrm{C} 19-\mathrm{C} 18$ & $83.15(13)$ \\
\hline $\mathrm{O} 9-\mathrm{S} 3-\mathrm{C} 19-\mathrm{C} 18$ & $-157.55(12)$ \\
\hline $\mathrm{C} 18-\mathrm{C} 19-\mathrm{C} 20-\mathrm{C} 15$ & $-4.2(2)$ \\
\hline $\mathrm{S} 3-\mathrm{C} 19-\mathrm{C} 20-\mathrm{C} 15$ & $174.65(11)$ \\
\hline $\mathrm{C} 16-\mathrm{C} 15-\mathrm{C} 20-\mathrm{C} 19$ & $-1.4(2)$ \\
\hline $\mathrm{C} 14-\mathrm{C} 15-\mathrm{C} 20-\mathrm{C} 19$ & $178.45(13)$ \\
\hline $\mathrm{N} 5-\mathrm{N} 6-\mathrm{C} 21-\mathrm{C} 22$ & $-169.89(13)$ \\
\hline $\mathrm{N} 5-\mathrm{N} 6-\mathrm{C} 21-\mathrm{C} 26$ & $10.6(2)$ \\
\hline $\mathrm{C} 26-\mathrm{C} 21-\mathrm{C} 22-\mathrm{C} 23$ & $0.9(2)$ \\
\hline $\mathrm{N} 6-\mathrm{C} 21-\mathrm{C} 22-\mathrm{C} 23$ & $-178.63(14)$ \\
\hline $\mathrm{C} 21-\mathrm{C} 22-\mathrm{C} 23-\mathrm{C} 24$ & $0.1(2)$ \\
\hline $\mathrm{C} 22-\mathrm{C} 23-\mathrm{C} 24-\mathrm{C} 25$ & $-0.7(2)$ \\
\hline $\mathrm{C} 23-\mathrm{C} 24-\mathrm{C} 25-\mathrm{C} 26$ & $0.5(3)$ \\
\hline $\mathrm{C} 24-\mathrm{C} 25-\mathrm{C} 26-\mathrm{C} 21$ & $0.4(2)$ \\
\hline $\mathrm{C} 22-\mathrm{C} 21-\mathrm{C} 26-\mathrm{C} 25$ & $-1.1(2)$ \\
\hline $\mathrm{N} 6-\mathrm{C} 21-\mathrm{C} 26-\mathrm{C} 25$ & $178.40(14)$ \\
\hline
\end{tabular}

Symmetry code: (i) $-x,-y+1,-z$.

Hydrogen-bond geometry $\left(A,{ }^{\circ}\right)$

\begin{tabular}{lllll}
\hline$D-\mathrm{H} \cdots A$ & $D-\mathrm{H}$ & $\mathrm{H} \cdots A$ & $D \cdots A$ & $D-\mathrm{H} \cdots A$ \\
\hline $\mathrm{O} 1 W-\mathrm{H} 1 W \cdots \mathrm{O} 9^{\mathrm{ii}}$ & $0.88(3)$ & $1.99(3)$ & $2.8404(16)$ & $163(2)$ \\
$\mathrm{O} 1 W-\mathrm{H} 2 W \cdots \mathrm{O} 1^{\mathrm{ii}}$ & $0.91(3)$ & $2.24(3)$ & $2.8830(17)$ & $127(2)$ \\
$\mathrm{O} 1 W-\mathrm{H} 2 W \cdots \mathrm{N} 2^{\mathrm{ii}}$ & $0.91(3)$ & $2.07(3)$ & $2.8972(18)$ & $150(2)$ \\
$\mathrm{O} 2 W-\mathrm{H} 3 W \cdots \mathrm{O} 99^{\mathrm{ii}}$ & $0.86(3)$ & $2.05(3)$ & $2.8852(18)$ & $163(2)$ \\
$\mathrm{O} 2 W-\mathrm{H} 4 W \cdots \mathrm{O} 6^{\mathrm{iii}}$ & $0.92(4)$ & $1.98(4)$ & $2.8932(18)$ & $174(3)$ \\
$\mathrm{O} 3 W-\mathrm{H} 5 W \cdots \mathrm{O} 5^{\mathrm{iii}}$ & $0.86(3)$ & $1.98(3)$ & $2.8320(18)$ & $178(3)$ \\
$\mathrm{O} 3 W-\mathrm{H} 6 W \cdots \mathrm{O} 5 W$ & $0.83(4)$ & $2.18(3)$ & $2.736(3)$ & $124(3)$
\end{tabular}




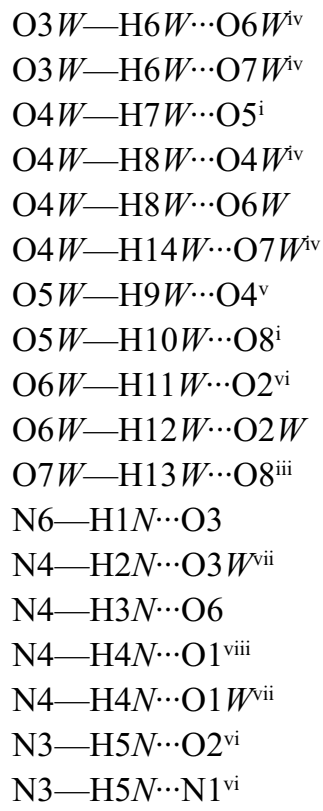

ymmetry codes: (i) $-x,-y+1,-z$; (ii) $-x$, $-y+1 / 2, z+1 / 2$; (viii) $-x+1,-y+1,-z+1$.

(V)

\section{Crystal data}

\section{$\mathrm{C}_{15} \mathrm{H}_{16} \mathrm{~N}_{6} \mathrm{O}_{2} \mathrm{~S}$}

$M_{r}=344.40$

Monoclinic, $P 2_{1} / c$

$a=8.5796(2) \AA$

$b=19.0371(5) \AA$

$c=11.2512(4) \AA$

$\beta=121.116(3)^{\circ}$

$V=1573.27(9) \AA^{3}$

$Z=4$

\section{Data collection}

Oxford Diffraction Xcalibur E diffractometer

Radiation source: sealed tube $\omega$ scans

$\begin{array}{llll}0.83(4) & 2.09(4) & 2.853(3) & 153(3) \\ 0.83(4) & 2.06(4) & 2.799(3) & 148(3) \\ 0.88(1) & 2.24(1) & 3.1093(19) & 169(2) \\ 0.88(1) & 2.00(2) & 2.825(3) & 154(4) \\ 0.88(1) & 2.41(5) & 2.871(3) & 113(4) \\ 0.89(1) & 2.16(3) & 2.902(3) & 140(4) \\ 0.88(1) & 1.85(1) & 2.710(3) & 166(4) \\ 0.89(1) & 1.99(1) & 2.843(3) & 163(3) \\ 0.89(1) & 2.22(2) & 2.893(3) & 132(2) \\ 0.88(1) & 1.85(1) & 2.731(3) & 178(5) \\ 0.88(1) & 1.91(2) & 2.699(3) & 150(3) \\ 0.92(2) & 1.75(2) & 2.5342(16) & 142(2) \\ 0.92(3) & 1.93(3) & 2.839(2) & 171(2) \\ 0.91(2) & 1.92(2) & 2.8245(18) & 170(2) \\ 0.91(2) & 2.01(2) & 2.8784(18) & 159.6(19) \\ 0.91(2) & 2.50(2) & 2.9386(19) & 110.5(16) \\ 0.84(2) & 2.57(2) & 3.0827(17) & 120.3(18) \\ 0.84(2) & 2.02(2) & 2.8655(19) & 177(2)\end{array}$

$F(000)=720$

$D_{\mathrm{x}}=1.454 \mathrm{Mg} \mathrm{m}^{-3}$

Mo $K \alpha$ radiation, $\lambda=0.71073 \AA$

Cell parameters from 8493 reflections

$\theta=3.0-29.5^{\circ}$

$\mu=0.23 \mathrm{~mm}^{-1}$

$T=123 \mathrm{~K}$

Sheet, colourless

$0.4 \times 0.3 \times 0.02 \mathrm{~mm}$

Absorption correction: multi-scan CrysAlis PRO, Oxford Diffraction Ltd., Version 1.171.34.40 (release 27-08-2010 CrysAlis 171 .NET) (compiled Aug 27 2010,11:50:40) Empirical absorption correction using spherical harmonics, implemented in SCALE3 ABSPACK scaling algorithm.

$T_{\min }=0.527, T_{\max }=1.000$ 22193 measured reflections 4082 independent reflections 3225 reflections with $I>2 \sigma(I)$

$R_{\text {int }}=0.040$

$\theta_{\text {max }}=29.0^{\circ}, \theta_{\min }=3.0^{\circ}$

$h=-11 \rightarrow 11$

$k=-25 \rightarrow 25$

$l=-14 \rightarrow 15$ 


\section{Refinement}

Refinement on $F^{2}$

Least-squares matrix: full

$R\left[F^{2}>2 \sigma\left(F^{2}\right)\right]=0.045$

$w R\left(F^{2}\right)=0.114$

$S=1.04$

4082 reflections

237 parameters

0 restraints

Special details

Geometry. All e.s.d.'s (except the e.s.d. in the dihedral angle between two 1.s. planes) are estimated using the full covariance matrix. The cell e.s.d.'s are taken into account individually in the estimation of e.s.d.'s in distances, angles and torsion angles; correlations between e.s.d.'s in cell parameters are only used when they are defined by crystal symmetry. An approximate (isotropic) treatment of cell e.s.d.'s is used for estimating e.s.d.'s involving 1.s. planes.

Fractional atomic coordinates and isotropic or equivalent isotropic displacement parameters $\left(\AA^{2}\right)$

\begin{tabular}{|c|c|c|c|c|}
\hline & $x$ & $y$ & $z$ & $U_{\text {iso }} * / U_{\text {eq }}$ \\
\hline $\mathrm{S} 1$ & $0.87444(6)$ & $0.84851(2)$ & $0.27777(4)$ & $0.02297(12)$ \\
\hline $\mathrm{O} 1$ & $0.97267(17)$ & $0.79251(7)$ & $0.25677(14)$ & 0.0309 (3) \\
\hline $\mathrm{O} 2$ & $0.96376(16)$ & $0.87572(7)$ & $0.41782(12)$ & $0.0271(3)$ \\
\hline N1 & $0.83358(19)$ & $0.90544(7)$ & $0.16260(15)$ & 0.0233 \\
\hline N2 & $0.73473(18)$ & $1.01144(7)$ & $0.05571(14)$ & $0.0216(3)$ \\
\hline N3 & $0.71259(19)$ & $0.98683(8)$ & $0.25488(15)$ & 0.0239 \\
\hline N4 & $0.1507(2)$ & $0.73439(9)$ & $0.12033(18)$ & $0.0284(3)$ \\
\hline N5 & $1.20543(19)$ & $1.03376(7)$ & $0.15032(15)$ & $0.0231(3)$ \\
\hline N6 & $1.0916(3)$ & $1.14087(10)$ & $0.0502(2)$ & $0.0411(4)$ \\
\hline $\mathrm{C} 1$ & $0.7592(2)$ & $0.96881(9)$ & $0.16105(17)$ & $0.0209(3)$ \\
\hline $\mathrm{C} 2$ & $0.6662(2)$ & $1.07527(9)$ & $0.04854(17)$ & 0.0238 \\
\hline $\mathrm{H} 2$ & 0.6475 & 1.1057 & -0.0247 & $0.029 *$ \\
\hline $\mathrm{C} 3$ & $0.6214(2)$ & $1.09897(9)$ & $0.14288(18)$ & $0.0258(4)$ \\
\hline $\mathrm{H} 3$ & 0.5754 & 1.1449 & 0.1382 & $0.031 *$ \\
\hline $\mathrm{C} 4$ & $0.6476(2)$ & $1.05165(9)$ & $0.24444(18)$ & $0.0258(4)$ \\
\hline $\mathrm{H} 4$ & 0.6175 & 1.0661 & 0.3110 & $0.031 *$ \\
\hline $\mathrm{C} 5$ & $0.6654(2)$ & $0.81161(8)$ & 0.23937 (17) & 0.0215 \\
\hline C6 & $0.5823(2)$ & $0.76230(9)$ & $0.13303(18)$ & $0.0266(4)$ \\
\hline H6 & 0.6422 & 0.7470 & 0.0864 & $0.032 *$ \\
\hline $\mathrm{C} 7$ & 0.4129 & $0.73551(9)$ & $0.09498(18)$ & $0.0275(4)$ \\
\hline $\mathrm{H} 7$ & 0.3577 & 0.7015 & 0.0230 & $0.033 *$ \\
\hline $\mathrm{C} 8$ & $0.3216(2)$ & $0.75825(8)$ & $0.16202(17)$ & 0.0219 \\
\hline $\mathrm{C} 9$ & $0.4076(2)$ & $0.80709(9)$ & $0.26908(17)$ & 0.0235 \\
\hline H9 & 0.3488 & 0.8224 & 0.3164 & $0.028^{*}$ \\
\hline $\mathrm{C} 10$ & $0.5776(2)$ & $0.83359(9)$ & $0.30745(17)$ & 0.0235 \\
\hline $\mathrm{H} 10$ & 0.6342 & 0.8669 & 0.3806 & $0.028^{*}$ \\
\hline $\mathrm{C} 11$ & $1.1346(2)$ & $1.09647(9)$ & $0.15389(18)$ & $0.0251(4)$ \\
\hline $\mathrm{C} 12$ & $1.1082(2)$ & $1.10992(10)$ & $0.2655(2)$ & $0.0316(4)$ \\
\hline H12 & 1.0611 & 1.1540 & 0.2721 & $0.038^{*}$ \\
\hline $\mathrm{C} 13$ & $1.1499(3)$ & $1.06005(11)$ & $0.3633(2)$ & $0.0340(4)$ \\
\hline
\end{tabular}

Hydrogen site location: mixed

$\mathrm{H}$ atoms treated by a mixture of independent

and constrained refinement

$w=1 /\left[\sigma^{2}\left(F_{\mathrm{o}}^{2}\right)+(0.0493 P)^{2}+0.8192 P\right]$

where $P=\left(F_{\mathrm{o}}{ }^{2}+2 F_{\mathrm{c}}{ }^{2}\right) / 3$

$(\Delta / \sigma)_{\max }=0.001$

$\Delta \rho_{\max }=0.39 \mathrm{e}^{-3}$

$\Delta \rho_{\min }=-0.48$ e $\AA^{-3}$ 


\begin{tabular}{|c|c|c|c|c|c|c|}
\hline H13 & \multirow{2}{*}{\multicolumn{2}{|c|}{$\begin{array}{l}1.1283 \\
1.2247(3)\end{array}$}} & & 0.4367 & \multicolumn{2}{|l|}{$0.041^{*}$} \\
\hline $\mathrm{C} 14$ & & & $0.99557(11)$ & $0.3572(2)$ & \multicolumn{2}{|l|}{$0.0368(5)$} \\
\hline H14 & \multicolumn{2}{|l|}{1.2565} & 0.9607 & 0.4264 & \multicolumn{2}{|l|}{$0.044 *$} \\
\hline $\mathrm{C} 15$ & \multicolumn{2}{|l|}{$1.2502(3)$} & $0.98455(10)$ & $0.2496(2)$ & \multicolumn{2}{|l|}{$0.0313(4)$} \\
\hline H15 & \multicolumn{2}{|l|}{1.3008} & 0.9412 & 0.2437 & \multicolumn{2}{|l|}{$0.038 *$} \\
\hline $\mathrm{H} 1 \mathrm{~N}$ & \multicolumn{2}{|l|}{0.109 (3) } & $0.6980(12)$ & $0.067(2)$ & \multicolumn{2}{|l|}{$0.036(6)^{*}$} \\
\hline $\mathrm{H} 2 \mathrm{~N}$ & \multicolumn{2}{|l|}{$0.100(3)$} & $0.7493(12)$ & $0.165(2)$ & \multicolumn{2}{|l|}{$0.038(6)^{*}$} \\
\hline $\mathrm{H} 3 \mathrm{~N}$ & \multicolumn{2}{|l|}{$1.220(3)$} & $1.0215(12)$ & $0.078(2)$ & \multicolumn{2}{|l|}{$0.044(6)^{*}$} \\
\hline $\mathrm{H} 4 \mathrm{~N}$ & \multicolumn{2}{|l|}{$1.045(3)$} & $1.1818(14)$ & $0.050(3)$ & \multicolumn{2}{|l|}{$0.051(7)^{*}$} \\
\hline $\mathrm{H} 5 \mathrm{~N}$ & \multicolumn{2}{|l|}{1.105 (4) } & $1.1256(13)$ & -0.021 & $0.060(8)^{*}$ & \\
\hline Atomi & nlacement naran & eters $\left(\AA^{2}\right)$ & & & & \\
\hline 1193 & $U^{11}$ & $U^{22}$ & $U^{33}$ & $U^{12}$ & $U^{13}$ & $U^{23}$ \\
\hline 1194 & 0.0214 (2) & $0.0248(2)$ & 0.0239 (2) & $0.00462(16)$ & 0.01254 (17) & $0.00161(16)$ \\
\hline 1195 & $0.0288(7)$ & $0.0315(7)$ & $0.0364(7)$ & $0.0096(5)$ & $0.0197(6)$ & $0.0023(6)$ \\
\hline 1196 & $0.0236(6)$ & $0.0309(7)$ & $0.0230(6)$ & $0.0037(5)$ & $0.0092(5)$ & $0.0021(5)$ \\
\hline 1197 & $0.0221(7)$ & $0.0251(7)$ & $0.0242(7)$ & $0.0019(6)$ & $0.0130(6)$ & $0.0013(6)$ \\
\hline 1198 & 0.0188 (7) & $0.0236(7)$ & $0.0215(7)$ & $-0.0033(5)$ & $0.0097(6)$ & -0.0017 (5) \\
\hline 1199 & $0.0220(7)$ & $0.0278(7)$ & $0.0224(7)$ & $0.0011(6)$ & 0.0119 (6) & $0.0000(6)$ \\
\hline 1200 & $0.0302(8)$ & $0.0267(8)$ & $0.0333(9)$ & $-0.0041(7)$ & $0.0201(7)$ & $-0.0066(7)$ \\
\hline 1201 & $0.0230(7)$ & $0.0257(7)$ & $0.0230(7)$ & $0.0009(6)$ & 0.0135 (6) & $-0.0012(6)$ \\
\hline 1202 & $0.0551(12)$ & $0.0302(9)$ & $0.0434(11)$ & $0.0162(8)$ & $0.0292(10)$ & $0.0084(8)$ \\
\hline 1203 & $0.0161(7)$ & $0.0247(8)$ & $0.0203(8)$ & $-0.0027(6)$ & $0.0082(6)$ & $-0.0017(6)$ \\
\hline 1204 & $0.0219(8)$ & $0.0241(8)$ & $0.0222(8)$ & $-0.0031(7)$ & $0.0092(7)$ & $0.0001(7)$ \\
\hline 1205 & $0.0235(8)$ & $0.0219(8)$ & $0.0267(9)$ & $-0.0007(7)$ & $0.0092(7)$ & $-0.0045(7)$ \\
\hline 1206 & $0.0215(8)$ & $0.0300(9)$ & $0.0247(8)$ & $-0.0001(7)$ & $0.0111(7)$ & $-0.0047(7)$ \\
\hline 1207 & $0.0238(8)$ & $0.0199(8)$ & $0.0227(8)$ & $0.0031(6)$ & $0.0135(7)$ & $0.0024(6)$ \\
\hline 1208 & $0.0310(9)$ & $0.0265(8)$ & $0.0288(9)$ & $0.0033(7)$ & $0.0201(8)$ & $-0.0030(7)$ \\
\hline 1209 & $0.0322(9)$ & $0.0250(8)$ & $0.0270(9)$ & $-0.0013(7)$ & $0.0166(8)$ & $-0.0071(7)$ \\
\hline 1210 & $0.0261(8)$ & $0.0183(7)$ & $0.0232(8)$ & $0.0027(6)$ & $0.0141(7)$ & $0.0044(6)$ \\
\hline 1211 & $0.0304(9)$ & $0.0229(8)$ & $0.0234(8)$ & $0.0022(7)$ & $0.0183(7)$ & 0.0015 (7) \\
\hline $\mathrm{C} 10$ & $0.0293(9)$ & $0.0228(8)$ & $0.0203(8)$ & $0.0014(7)$ & $0.0142(7)$ & $-0.0009(6)$ \\
\hline C11 & $0.0195(8)$ & $0.0253(8)$ & $0.0286(9)$ & $0.0006(7)$ & $0.0111(7)$ & $-0.0029(7)$ \\
\hline C12 & 0.0248 (9) & $0.0353(10)$ & $0.0367(10)$ & $-0.0030(8)$ & $0.0173(8)$ & $-0.0133(8)$ \\
\hline $\mathrm{C} 13$ & $0.0268(9)$ & $0.0514(12)$ & $0.0281(9)$ & $-0.0147(9)$ & $0.0173(8)$ & -0.0168 (9) \\
\hline C14 & 0.0405 (11) & $0.0416(11)$ & $0.0289(10)$ & -0.0055 (9) & $0.0185(9)$ & $0.0039(8)$ \\
\hline $\mathrm{C} 15$ & $0.0368(10)$ & $0.0270(9)$ & $0.0331(10)$ & $0.0041(8)$ & $0.0202(9)$ & $0.0033(8)$ \\
\hline
\end{tabular}

Geometric parameters $\left(\AA,{ }^{\circ}\right)$

\begin{tabular}{llll}
\hline $\mathrm{S} 1-\mathrm{O} 2$ & $1.4458(13)$ & $\mathrm{C} 3-\mathrm{H} 3$ & 0.9500 \\
$\mathrm{~S} 1-\mathrm{O} 1$ & $1.4520(12)$ & $\mathrm{C} 4-\mathrm{H} 4$ & 0.9500 \\
$\mathrm{~S} 1-\mathrm{N} 1$ & $1.5834(15)$ & $\mathrm{C} 5-\mathrm{C} 10$ & $1.388(2)$ \\
$\mathrm{S} 1-\mathrm{C} 5$ & $1.7592(17)$ & $\mathrm{C} 5-\mathrm{C} 6$ & $1.393(2)$ \\
$\mathrm{N} 1-\mathrm{C} 1$ & $1.361(2)$ & $\mathrm{C} 6-\mathrm{C} 7$ & $1.383(3)$ \\
$\mathrm{N} 2-\mathrm{C} 2$ & $1.334(2)$ & $\mathrm{C} 6-\mathrm{H} 6$ & 0.9500 \\
$\mathrm{~N} 2-\mathrm{C} 1$ & $1.360(2)$ & $\mathrm{C} 7-\mathrm{C} 8$ & $1.407(2)$
\end{tabular}




\begin{tabular}{|c|c|c|c|c|}
\hline 1226 & $\mathrm{~N} 3-\mathrm{C} 4$ & $1.334(2)$ & $\mathrm{C} 7-\mathrm{H} 7$ & 0.9500 \\
\hline 1227 & $\mathrm{~N} 3-\mathrm{C} 1$ & $1.352(2)$ & $\mathrm{C} 8-\mathrm{C} 9$ & $1.393(2)$ \\
\hline 1228 & $\mathrm{~N} 4-\mathrm{C} 8$ & $1.366(2)$ & $\mathrm{C} 9-\mathrm{C} 10$ & $1.384(2)$ \\
\hline 1229 & $\mathrm{~N} 4-\mathrm{H} 1 \mathrm{~N}$ & $0.86(2)$ & C9- $\mathrm{H} 9$ & 0.9500 \\
\hline 1230 & $\mathrm{~N} 4-\mathrm{H} 2 \mathrm{~N}$ & $0.87(2)$ & $\mathrm{C} 10-\mathrm{H} 10$ & 0.9500 \\
\hline 1231 & N5-C11 & $1.349(2)$ & $\mathrm{C} 11-\mathrm{C} 12$ & $1.410(3)$ \\
\hline 1232 & N5-C15 & $1.353(2)$ & $\mathrm{C} 12-\mathrm{C} 13$ & $1.354(3)$ \\
\hline 1233 & $\mathrm{~N} 5-\mathrm{H} 3 \mathrm{~N}$ & $0.92(2)$ & $\mathrm{C} 12-\mathrm{H} 12$ & 0.9500 \\
\hline 1234 & N6-C11 & $1.329(3)$ & $\mathrm{C} 13-\mathrm{C} 14$ & $1.403(3)$ \\
\hline 1235 & $\mathrm{~N} 6-\mathrm{H} 4 \mathrm{~N}$ & $0.87(3)$ & C13-H13 & 0.9500 \\
\hline 1236 & $\mathrm{~N} 6-\mathrm{H} 5 \mathrm{~N}$ & $0.92(3)$ & C14-C15 & $1.352(3)$ \\
\hline 1237 & $\mathrm{C} 2-\mathrm{C} 3$ & $1.379(2)$ & C14-H14 & 0.9500 \\
\hline 1238 & $\mathrm{C} 2-\mathrm{H} 2$ & 0.9500 & C15-H15 & 0.9500 \\
\hline 1239 & $\mathrm{C} 3-\mathrm{C} 4$ & $1.379(3)$ & & \\
\hline \multicolumn{5}{|l|}{1240} \\
\hline 1241 & $\mathrm{O} 2-\mathrm{S} 1-\mathrm{O} 1$ & $114.52(8)$ & $\mathrm{C} 6-\mathrm{C} 5-\mathrm{S} 1$ & 118.99 (13) \\
\hline 1242 & $\mathrm{O} 2-\mathrm{S} 1-\mathrm{N} 1$ & $114.53(8)$ & $\mathrm{C} 7-\mathrm{C} 6-\mathrm{C} 5$ & $120.28(16)$ \\
\hline 1243 & $\mathrm{O} 1-\mathrm{S} 1-\mathrm{N} 1$ & $105.72(8)$ & $\mathrm{C} 7-\mathrm{C} 6-\mathrm{H} 6$ & 119.9 \\
\hline 1244 & $\mathrm{O} 2-\mathrm{S} 1-\mathrm{C} 5$ & $108.04(8)$ & $\mathrm{C} 5-\mathrm{C} 6-\mathrm{H} 6$ & 119.9 \\
\hline 1245 & $\mathrm{O} 1-\mathrm{S} 1-\mathrm{C} 5$ & $105.70(8)$ & $\mathrm{C} 6-\mathrm{C} 7-\mathrm{C} 8$ & $120.50(16)$ \\
\hline 1246 & $\mathrm{~N} 1-\mathrm{S} 1-\mathrm{C} 5$ & $107.81(8)$ & $\mathrm{C} 6-\mathrm{C} 7-\mathrm{H} 7$ & 119.7 \\
\hline 1247 & $\mathrm{C} 1-\mathrm{N} 1-\mathrm{S} 1$ & $121.43(12)$ & $\mathrm{C} 8-\mathrm{C} 7-\mathrm{H} 7$ & 119.7 \\
\hline 1248 & $\mathrm{C} 2-\mathrm{N} 2-\mathrm{C} 1$ & $117.44(14)$ & $\mathrm{N} 4-\mathrm{C} 8-\mathrm{C} 9$ & $120.93(16)$ \\
\hline 1249 & $\mathrm{C} 4-\mathrm{N} 3-\mathrm{C} 1$ & $116.41(15)$ & $\mathrm{N} 4-\mathrm{C} 8-\mathrm{C} 7$ & $120.64(16)$ \\
\hline 1250 & $\mathrm{C} 8-\mathrm{N} 4-\mathrm{H} 1 \mathrm{~N}$ & $119.2(15)$ & $\mathrm{C} 9-\mathrm{C} 8-\mathrm{C} 7$ & $118.40(16)$ \\
\hline 1251 & $\mathrm{C} 8-\mathrm{N} 4-\mathrm{H} 2 \mathrm{~N}$ & $117.6(15)$ & $\mathrm{C} 10-\mathrm{C} 9-\mathrm{C} 8$ & $121.00(16)$ \\
\hline 1252 & $\mathrm{H} 1 \mathrm{~N}-\mathrm{N} 4-\mathrm{H} 2 \mathrm{~N}$ & $121(2)$ & $\mathrm{C} 10-\mathrm{C} 9-\mathrm{H} 9$ & 119.5 \\
\hline 1253 & $\mathrm{C} 11-\mathrm{N} 5-\mathrm{C} 15$ & $121.88(16)$ & $\mathrm{C} 8-\mathrm{C} 9-\mathrm{H} 9$ & 119.5 \\
\hline 1254 & $\mathrm{C} 11-\mathrm{N} 5-\mathrm{H} 3 \mathrm{~N}$ & $121.5(14)$ & $\mathrm{C} 9-\mathrm{C} 10-\mathrm{C} 5$ & $120.19(16)$ \\
\hline 1255 & $\mathrm{C} 15-\mathrm{N} 5-\mathrm{H} 3 \mathrm{~N}$ & $116.5(14)$ & $\mathrm{C} 9-\mathrm{C} 10-\mathrm{H} 10$ & 119.9 \\
\hline 1256 & $\mathrm{C} 11-\mathrm{N} 6-\mathrm{H} 4 \mathrm{~N}$ & $119.3(16)$ & $\mathrm{C} 5-\mathrm{C} 10-\mathrm{H} 10$ & 119.9 \\
\hline 1257 & $\mathrm{C} 11-\mathrm{N} 6-\mathrm{H} 5 \mathrm{~N}$ & $117.6(16)$ & N6-C11-N5 & $117.51(17)$ \\
\hline 1258 & $\mathrm{H} 4 \mathrm{~N}-\mathrm{N} 6-\mathrm{H} 5 \mathrm{~N}$ & $123(2)$ & $\mathrm{N} 6-\mathrm{C} 11-\mathrm{C} 12$ & $124.49(18)$ \\
\hline 1259 & $\mathrm{~N} 3-\mathrm{C} 1-\mathrm{N} 2$ & $123.66(15)$ & $\mathrm{N} 5-\mathrm{C} 11-\mathrm{C} 12$ & $117.99(17)$ \\
\hline 1260 & $\mathrm{~N} 3-\mathrm{C} 1-\mathrm{N} 1$ & $122.74(15)$ & $\mathrm{C} 13-\mathrm{C} 12-\mathrm{C} 11$ & $119.97(17)$ \\
\hline 1261 & $\mathrm{~N} 2-\mathrm{C} 1-\mathrm{N} 1$ & $113.60(14)$ & $\mathrm{C} 13-\mathrm{C} 12-\mathrm{H} 12$ & 120.0 \\
\hline 1262 & $\mathrm{~N} 2-\mathrm{C} 2-\mathrm{C} 3$ & $122.76(16)$ & $\mathrm{C} 11-\mathrm{C} 12-\mathrm{H} 12$ & 120.0 \\
\hline 1263 & $\mathrm{~N} 2-\mathrm{C} 2-\mathrm{H} 2$ & 118.6 & $\mathrm{C} 12-\mathrm{C} 13-\mathrm{C} 14$ & $120.61(17)$ \\
\hline 1264 & $\mathrm{C} 3-\mathrm{C} 2-\mathrm{H} 2$ & 118.6 & $\mathrm{C} 12-\mathrm{C} 13-\mathrm{H} 13$ & 119.7 \\
\hline 1265 & $\mathrm{C} 2-\mathrm{C} 3-\mathrm{C} 4$ & $115.67(16)$ & $\mathrm{C} 14-\mathrm{C} 13-\mathrm{H} 13$ & 119.7 \\
\hline 1266 & $\mathrm{C} 2-\mathrm{C} 3-\mathrm{H} 3$ & 122.2 & $\mathrm{C} 15-\mathrm{C} 14-\mathrm{C} 13$ & $117.98(18)$ \\
\hline 1267 & $\mathrm{C} 4-\mathrm{C} 3-\mathrm{H} 3$ & 122.2 & $\mathrm{C} 15-\mathrm{C} 14-\mathrm{H} 14$ & 121.0 \\
\hline 1268 & $\mathrm{~N} 3-\mathrm{C} 4-\mathrm{C} 3$ & $123.98(16)$ & $\mathrm{C} 13-\mathrm{C} 14-\mathrm{H} 14$ & 121.0 \\
\hline 1269 & $\mathrm{~N} 3-\mathrm{C} 4-\mathrm{H} 4$ & 118.0 & $\mathrm{C} 14-\mathrm{C} 15-\mathrm{N} 5$ & $121.55(18)$ \\
\hline 1270 & $\mathrm{C} 3-\mathrm{C} 4-\mathrm{H} 4$ & 118.0 & $\mathrm{C} 14-\mathrm{C} 15-\mathrm{H} 15$ & 119.2 \\
\hline 1271 & $\mathrm{C} 10-\mathrm{C} 5-\mathrm{C} 6$ & $119.61(16)$ & N5-C15-H15 & 119.2 \\
\hline 1272 & $\mathrm{C} 10-\mathrm{C} 5-\mathrm{S} 1$ & $121.30(13)$ & & \\
\hline
\end{tabular}




$\begin{array}{ll}\mathrm{O} 2-\mathrm{S} 1-\mathrm{N} 1-\mathrm{C} 1 & -46.01(15) \\ \mathrm{O} 1-\mathrm{S} 1-\mathrm{N} 1-\mathrm{C} 1 & -173.05(13) \\ \mathrm{C} 5-\mathrm{S} 1-\mathrm{N} 1-\mathrm{C} 1 & 74.26(14) \\ \mathrm{C} 4-\mathrm{N} 3-\mathrm{C} 1-\mathrm{N} 2 & -3.3(2) \\ \mathrm{C} 4-\mathrm{N} 3-\mathrm{C} 1-\mathrm{N} 1 & 177.47(15) \\ \mathrm{C} 2-\mathrm{N} 2-\mathrm{C} 1-\mathrm{N} 3 & 2.2(2) \\ \mathrm{C} 2-\mathrm{N} 2-\mathrm{C} 1-\mathrm{N} 1 & -178.51(14) \\ \mathrm{S} 1-\mathrm{N} 1-\mathrm{C} 1-\mathrm{N} 3 & -1.2(2) \\ \mathrm{S} 1-\mathrm{N} 1-\mathrm{C} 1-\mathrm{N} 2 & 179.50(11) \\ \mathrm{C} 1-\mathrm{N} 2-\mathrm{C} 2-\mathrm{C} 3 & 0.4(2) \\ \mathrm{N} 2-\mathrm{C} 2-\mathrm{C} 3-\mathrm{C} 4 & -1.6(2) \\ \mathrm{C} 1-\mathrm{N} 3-\mathrm{C} 4-\mathrm{C} 3 & 2.0(2) \\ \mathrm{C} 2-\mathrm{C} 3-\mathrm{C} 4-\mathrm{N} 3 & 0.3(3) \\ \mathrm{O} 2-\mathrm{S} 1-\mathrm{C} 5-\mathrm{C} 10 & 24.88(16) \\ \mathrm{O} 1-\mathrm{S} 1-\mathrm{C} 5-\mathrm{C} 10 & 147.91(14) \\ \mathrm{N} 1-\mathrm{S} 1-\mathrm{C} 5-\mathrm{C} 10 & -99.39(15) \\ \mathrm{O} 2-\mathrm{S} 1-\mathrm{C} 5-\mathrm{C} 6 & -158.80(13) \\ \mathrm{O} 1-\mathrm{S} 1-\mathrm{C} 5-\mathrm{C} 6 & -35.78(16) \\ \mathrm{N} 1-\mathrm{S} 1-\mathrm{C} 5-\mathrm{C} 6 & 76.93(15)\end{array}$

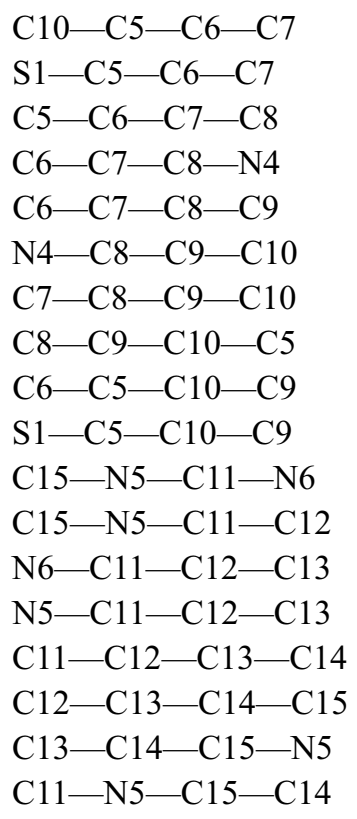

Hydrogen-bond geometry $\left(\AA,{ }^{\circ}\right)$

\begin{tabular}{lllll}
\hline$D-\mathrm{H} \cdots A$ & $D-\mathrm{H}$ & $\mathrm{H} \cdots A$ & $D \cdots A$ & $D-\mathrm{H} \cdots A$ \\
\hline $\mathrm{N} 4-\mathrm{H} 1 N \cdots \mathrm{O} 2^{\mathrm{i}}$ & $0.86(2)$ & $2.04(2)$ & $2.895(2)$ & $168(2)$ \\
$\mathrm{N} 4-\mathrm{H} 2 N \cdots \mathrm{O} 1^{\text {ii }}$ & $0.87(2)$ & $2.03(2)$ & $2.887(2)$ & $174(2)$ \\
$\mathrm{N} 5-\mathrm{H} 3 N \cdots \mathrm{N} 2^{\text {iii }}$ & $0.92(2)$ & $1.85(2)$ & $2.758(2)$ & $174(2)$ \\
$\mathrm{N} 6-\mathrm{H} 4 N \cdots \mathrm{N} 4^{\text {iv }}$ & $0.87(3)$ & $2.39(3)$ & $3.085(2)$ & $137(2)$ \\
$\mathrm{N} 6-\mathrm{H} 5 N \cdots \mathrm{N} 1^{\text {iii }}$ & $0.92(3)$ & $2.00(3)$ & $2.918(2)$ & $173(3)$ \\
\hline
\end{tabular}

Symmetry codes: (i) $x-1,-y+3 / 2, z-1 / 2$; (ii) $x-1, y, z$; (iii) $-x+2,-y+2,-z$; (iv) $-x+1,-y+2,-z$.

(VI)

\section{Crystal data}

$\mathrm{C}_{12} \mathrm{H}_{19} \mathrm{~N}_{5} \mathrm{O}_{4} \mathrm{~S}$

$M_{r}=329.38$

Monoclinic, $P 2_{1} / c$

$a=12.7755$ (4) $\AA$

$b=9.8979$ (4) $\AA$

$c=11.5366$ (4) $\AA$

$\beta=93.508(3)^{\circ}$

$V=1456.08(9) \AA^{3}$

$Z=4$

\section{Data collection}

Oxford Diffraction Xcalibur E diffractometer

Radiation source: sealed tube $\omega$ scans

\section{$F(000)=696$}

$D_{\mathrm{x}}=1.503 \mathrm{Mg} \mathrm{m}^{-3}$

Mo $K \alpha$ radiation, $\lambda=0.71073 \AA$

Cell parameters from 3829 reflections

$\theta=3.2-29.5^{\circ}$

$\mu=0.25 \mathrm{~mm}^{-1}$

$T=123 \mathrm{~K}$

Fragment, colourless

$0.25 \times 0.24 \times 0.12 \mathrm{~mm}$ 
Absorption correction: multi-scan CrysAlis PRO, Oxford Diffraction Ltd., Version 1.171.34.40 (release 27-08-2010 CrysAlis171 .NET) (compiled Aug 27 2010,11:50:40) Empirical absorption correction using spherical harmonics, implemented in SCALE3 ABSPACK scaling algorithm.

$T_{\min }=0.904, T_{\max }=1.000$

Refinement

Refinement on $F^{2}$

Least-squares matrix: full

$R\left[F^{2}>2 \sigma\left(F^{2}\right)\right]=0.037$

$w R\left(F^{2}\right)=0.095$

$S=1.06$

3571 reflections

231 parameters

0 restraints
7213 measured reflections

3571 independent reflections

2942 reflections with $I>2 \sigma(I)$

$R_{\text {int }}=0.023$

$\theta_{\max }=29.5^{\circ}, \theta_{\min }=3.2^{\circ}$

$h=-16 \rightarrow 17$

$k=-12 \rightarrow 12$

$l=-15 \rightarrow 15$

Hydrogen site location: mixed

$\mathrm{H}$ atoms treated by a mixture of independent and constrained refinement

$w=1 /\left[\sigma^{2}\left(F_{0}^{2}\right)+(0.0415 P)^{2}+0.609 P\right]$ where $P=\left(F_{\mathrm{o}}{ }^{2}+2 F_{\mathrm{c}}{ }^{2}\right) / 3$

$(\Delta / \sigma)_{\max }<0.001$

$\Delta \rho_{\max }=0.45 \mathrm{e}^{-3}$

$\Delta \rho_{\min }=-0.47$ e $\AA^{-3}$

\section{Special details}

Geometry. All e.s.d.'s (except the e.s.d. in the dihedral angle between two 1.s. planes) are estimated using the full covariance matrix. The cell e.s.d.'s are taken into account individually in the estimation of e.s.d.'s in distances, angles and torsion angles; correlations between e.s.d.'s in cell parameters are only used when they are defined by crystal symmetry. An approximate (isotropic) treatment of cell e.s.d.'s is used for estimating e.s.d.'s involving l.s. planes.

Fractional atomic coordinates and isotropic or equivalent isotropic displacement parameters $\left(\hat{A}^{2}\right)$

\begin{tabular}{|c|c|c|c|c|}
\hline & $x$ & $y$ & $z$ & $U_{\text {iso }} * / U_{\text {eq }}$ \\
\hline $\mathrm{S} 1$ & $0.22358(3)$ & $0.19320(4)$ & $0.61905(3)$ & $0.01162(11)$ \\
\hline $\mathrm{O} 1$ & $0.17136(8)$ & $0.31689(11)$ & $0.58001(10)$ & $0.0164(2)$ \\
\hline $\mathrm{O} 2$ & $0.20716(8)$ & $0.15689(11)$ & $0.73914(9)$ & $0.0150(2)$ \\
\hline $\mathrm{O} 3$ & $0.45324(9)$ & $-0.36122(12)$ & $0.56317(11)$ & $0.0195(3)$ \\
\hline $\mathrm{O} 1 \mathrm{~W}$ & $0.04450(10)$ & $0.06888(14)$ & $0.88727(13)$ & $0.0264(3)$ \\
\hline N1 & $0.34262(10)$ & $0.21715(13)$ & $0.59377(11)$ & $0.0131(3)$ \\
\hline N2 & $0.51428(10)$ & $0.15350(13)$ & $0.58746(11)$ & $0.0146(3)$ \\
\hline N3 & $0.39549(10)$ & $0.00832(13)$ & $0.68028(11)$ & $0.0150(3)$ \\
\hline N4 & $0.06716(12)$ & $-0.25029(16)$ & $0.31048(14)$ & $0.0204(3)$ \\
\hline N5 & $0.33848(11)$ & $-0.55735(15)$ & $0.42864(13)$ & 0.0153 \\
\hline $\mathrm{C} 1$ & 0.41755 (11) & $0.12192(16)$ & $0.62242(13)$ & $0.0125(3)$ \\
\hline $\mathrm{C} 2$ & $0.59122(12)$ & $0.06494(17)$ & $0.61312(14)$ & $0.0167(3)$ \\
\hline $\mathrm{H} 2$ & 0.6595 & 0.0845 & 0.5894 & $0.020 *$ \\
\hline $\mathrm{C} 3$ & $0.57580(13)$ & $-0.05364(17)$ & $0.67255(14)$ & $0.0178(3)$ \\
\hline H3 & 0.6312 & -0.1156 & 0.6906 & $0.021 *$ \\
\hline $\mathrm{C} 4$ & $0.47525(12)$ & $-0.07671(17)$ & $0.70408(14)$ & 0.0169 \\
\hline H4 & 0.4617 & -0.1575 & 0.7450 & $0.020 *$ \\
\hline $\mathrm{C} 5$ & $0.17356(11)$ & $0.06143(15)$ & $0.53090(13)$ & 0.0119 \\
\hline C6 & $0.14954(12)$ & $-0.06369(16)$ & $0.57665(14)$ & 0.0149 \\
\hline H6 & 0.1589 & -0.0789 & 0.6579 & $0.018 *$ \\
\hline $\mathrm{C} 7$ & $0.11201(12)$ & $-0.16636(17)$ & $0.50398(14)$ & $0.0163(3)$ \\
\hline H7 & 0.0946 & -0.2513 & 0.5359 & $0.020^{*}$ \\
\hline $\mathrm{C} 8$ & 0.09943 (11) & $-0.14617(16)$ & $0.38370(14)$ & $0.0150(3)$ \\
\hline
\end{tabular}




$\begin{array}{lll}\text { C9 } & 0.12428(12) & -0.01923(16) \\ \text { H9 } & 0.1163 & -0.0040 \\ \text { C10 } & 0.16023(11) & 0.08336(16) \\ \text { H10 } & 0.1760 & 0.1693 \\ \text { C11 } & 0.34327(12) & -0.37296(17) \\ \text { H11A } & 0.3136 & -0.2833 \\ \text { H11B } & 0.3284 & -0.4349 \\ \text { C12 } & 0.29135(12) & -0.42582(16) \\ \text { H12A } & 0.2153 & -0.4374 \\ \text { H12B } & 0.3005 & -0.3601 \\ \text { H1N } & 0.0376(17) & -0.223(2) \\ \text { H2N } & 0.0370(17) & -0.313(2) \\ \text { H3N } & 0.4051(17) & -0.550(2) \\ \text { H4N } & 0.3120(17) & -0.587(2) \\ \text { H5N } & 0.3293(17) & -0.625(2) \\ \text { H1H } & 0.4656(19) & -0.297(3) \\ \text { H1W } & 0.095(2) & 0.097(3) \\ \text { H2W } & 0.070(2) & 0.106(3)\end{array}$

$0.33835(13)$
0.2570
$0.41125(13)$
0.3800
$0.57033(15)$
0.5878
0.6347
$0.45860(15)$
0.4673
0.3953
$0.243(2)$
$0.3425(19)$
$0.4209(17)$
$0.360(2)$
$0.483(2)$
$0.518(2)$
$0.833(3)$
$0.950(2)$

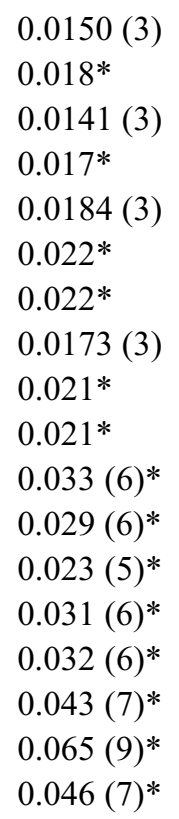

Atomic displacement parameters $\left(\AA^{2}\right)$

1373

1374

1375

1376

1377

1378

1379

1380

1381

1382

1383

1384

1385

1386

1387

1388

1389

1390

1391

1392

1393

1394

1395

\begin{tabular}{|c|c|c|c|c|c|c|}
\hline & $U^{11}$ & $U^{22}$ & $U^{33}$ & $U^{12}$ & $U^{13}$ & $U^{23}$ \\
\hline $\mathrm{S} 1$ & $0.01262(17)$ & 0.01188 (19) & $0.01020(19)$ & $0.00106(13)$ & $-0.00062(13)$ & $-0.00008(14)$ \\
\hline $\mathrm{O} 1$ & $0.0180(5)$ & $0.0138(6)$ & $0.0170(6)$ & $0.0038(4)$ & $-0.0017(4)$ & $0.0010(5)$ \\
\hline $\mathrm{O} 2$ & $0.0167(5)$ & $0.0185(6)$ & $0.0099(5)$ & $0.0023(4)$ & $0.0007(4)$ & $0.0001(4)$ \\
\hline $\mathrm{O} 3$ & $0.0162(5)$ & $0.0161(6)$ & $0.0254(7)$ & $-0.0015(5)$ & $-0.0037(5)$ & $0.0028(5)$ \\
\hline $\mathrm{O} 1 \mathrm{~W}$ & $0.0229(6)$ & $0.0330(8)$ & $0.0227(7)$ & $-0.0010(5)$ & $-0.0029(5)$ & $-0.0036(6)$ \\
\hline N1 & $0.0127(6)$ & $0.0129(6)$ & $0.0135(6)$ & $0.0000(5)$ & $-0.0011(5)$ & $0.0009(5)$ \\
\hline N2 & $0.0147(6)$ & $0.0149(7)$ & $0.0139(6)$ & $-0.0009(5)$ & $-0.0006(5)$ & $-0.0016(5)$ \\
\hline N3 & $0.0179(6)$ & $0.0144(7)$ & $0.0126(6)$ & $0.0016(5)$ & $0.0012(5)$ & $0.0008(5)$ \\
\hline N4 & $0.0253(7)$ & $0.0185(8)$ & $0.0171(8)$ & $-0.0046(6)$ & $-0.0014(6)$ & $-0.0029(6)$ \\
\hline N5 & $0.0164(7)$ & $0.0161(7)$ & $0.0131(7)$ & $-0.0015(5)$ & $-0.0010(5)$ & $0.0014(6)$ \\
\hline $\mathrm{C} 1$ & $0.0149(7)$ & $0.0137(7)$ & $0.0085(7)$ & $-0.0005(6)$ & $-0.0017(5)$ & $-0.0032(6)$ \\
\hline $\mathrm{C} 2$ & $0.0135(7)$ & $0.0206(8)$ & $0.0158(8)$ & $0.0009(6)$ & $0.0002(6)$ & $-0.0049(7)$ \\
\hline $\mathrm{C} 3$ & $0.0199(8)$ & $0.0184(8)$ & $0.0148(8)$ & $0.0061(6)$ & $-0.0016(6)$ & $-0.0017(6)$ \\
\hline $\mathrm{C} 4$ & $0.0222(8)$ & $0.0147(8)$ & $0.0136(8)$ & $0.0026(6)$ & $-0.0004(6)$ & $-0.0002(6)$ \\
\hline $\mathrm{C} 5$ & $0.0096(6)$ & $0.0138(7)$ & $0.0122(7)$ & $0.0000(5)$ & $0.0000(5)$ & $-0.0008(6)$ \\
\hline C6 & $0.0149(7)$ & $0.0188(8)$ & $0.0109(7)$ & $0.0001(6)$ & $0.0013(5)$ & $0.0010(6)$ \\
\hline $\mathrm{C} 7$ & $0.0172(7)$ & $0.0155(8)$ & $0.0161(8)$ & $-0.0031(6)$ & $0.0017(6)$ & $0.0012(6)$ \\
\hline $\mathrm{C} 8$ & $0.0111(7)$ & $0.0170(8)$ & $0.0169(8)$ & $0.0007(6)$ & $0.0007(6)$ & $-0.0036(6)$ \\
\hline $\mathrm{C} 9$ & $0.0152(7)$ & $0.0194(8)$ & $0.0104(7)$ & $0.0022(6)$ & $0.0003(6)$ & $0.0003(6)$ \\
\hline $\mathrm{C} 10$ & $0.0135(7)$ & $0.0154(7)$ & $0.0134(7)$ & $0.0008(6)$ & $0.0007(5)$ & $0.0026(6)$ \\
\hline C11 & $0.0171(7)$ & $0.0202(8)$ & $0.0178(8)$ & $-0.0006(6)$ & $0.0000(6)$ & $-0.0022(7)$ \\
\hline $\mathrm{C} 12$ & $0.0168(7)$ & $0.0159(8)$ & $0.0187(8)$ & $-0.0007(6)$ & $-0.0021(6)$ & $-0.0002(7)$ \\
\hline
\end{tabular}


1400

1401

1402

1403

1404

1405

1406

1407

1408

1409

1410

1411

1412

1413

1414

1415

1416

1417

1418

1419

1420

1421

1422

1423

1424

1425

1426

1427

1428

1429

1430

1431

1432

1433

1434

1435

1436

1437

1438

1439

1440

1441

1442

Geometric parameters $\left(\AA,{ }^{\circ}\right)$

\begin{tabular}{|c|c|c|c|}
\hline $\mathrm{S} 1-\mathrm{O} 1$ & $1.4525(11)$ & $\mathrm{C} 2-\mathrm{C} 3$ & $1.379(2)$ \\
\hline $\mathrm{S} 1-\mathrm{O} 2$ & $1.4588(11)$ & $\mathrm{C} 2-\mathrm{H} 2$ & 0.9500 \\
\hline $\mathrm{S} 1-\mathrm{N} 1$ & $1.5838(13)$ & $\mathrm{C} 3-\mathrm{C} 4$ & $1.376(2)$ \\
\hline $\mathrm{S} 1-\mathrm{C} 5$ & $1.7505(15)$ & $\mathrm{C} 3-\mathrm{H} 3$ & 0.9500 \\
\hline $\mathrm{O} 3-\mathrm{C} 11$ & $1.4172(19)$ & $\mathrm{C} 4-\mathrm{H} 4$ & 0.9500 \\
\hline $\mathrm{O} 3-\mathrm{H} 1 \mathrm{H}$ & $0.84(3)$ & $\mathrm{C} 5-\mathrm{C} 6$ & $1.388(2)$ \\
\hline $\mathrm{O} 1 \mathrm{~W}-\mathrm{H} 1 \mathrm{~W}$ & $0.97(3)$ & $\mathrm{C} 5-\mathrm{C} 10$ & $1.397(2)$ \\
\hline $\mathrm{O} 1 \mathrm{~W}-\mathrm{H} 2 \mathrm{~W}$ & $0.86(3)$ & $\mathrm{C} 6-\mathrm{C} 7$ & $1.384(2)$ \\
\hline $\mathrm{N} 1-\mathrm{C} 1$ & $1.3694(19)$ & $\mathrm{C} 6-\mathrm{H} 6$ & 0.9500 \\
\hline $\mathrm{N} 2-\mathrm{C} 2$ & $1.337(2)$ & $\mathrm{C} 7-\mathrm{C} 8$ & $1.401(2)$ \\
\hline $\mathrm{N} 2-\mathrm{C} 1$ & $1.3596(19)$ & $\mathrm{C} 7-\mathrm{H} 7$ & 0.9500 \\
\hline $\mathrm{N} 3-\mathrm{C} 4$ & $1.337(2)$ & $\mathrm{C} 8-\mathrm{C} 9$ & $1.405(2)$ \\
\hline $\mathrm{N} 3-\mathrm{C} 1$ & $1.346(2)$ & $\mathrm{C} 9-\mathrm{C} 10$ & $1.379(2)$ \\
\hline $\mathrm{N} 4-\mathrm{C} 8$ & $1.379(2)$ & $\mathrm{C} 9-\mathrm{H} 9$ & 0.9500 \\
\hline $\mathrm{N} 4-\mathrm{H} 1 \mathrm{~N}$ & $0.89(2)$ & $\mathrm{C} 10-\mathrm{H} 10$ & 0.9500 \\
\hline $\mathrm{N} 4-\mathrm{H} 2 \mathrm{~N}$ & $0.83(2)$ & $\mathrm{C} 11-\mathrm{C} 12$ & $1.507(2)$ \\
\hline $\mathrm{N} 5-\mathrm{C} 12$ & $1.484(2)$ & $\mathrm{C} 11-\mathrm{H} 11 \mathrm{~A}$ & 0.9900 \\
\hline $\mathrm{N} 5-\mathrm{H} 3 \mathrm{~N}$ & $0.86(2)$ & $\mathrm{C} 11-\mathrm{H} 11 \mathrm{~B}$ & 0.9900 \\
\hline $\mathrm{N} 5-\mathrm{H} 4 \mathrm{~N}$ & $0.90(2)$ & $\mathrm{C} 12-\mathrm{H} 12 \mathrm{~A}$ & 0.9900 \\
\hline $\mathrm{N} 5-\mathrm{H} 5 \mathrm{~N}$ & $0.93(2)$ & $\mathrm{C} 12-\mathrm{H} 12 \mathrm{~B}$ & 0.9900 \\
\hline $\mathrm{O} 1-\mathrm{S} 1-\mathrm{O} 2$ & $114.34(7)$ & $\mathrm{C} 3-\mathrm{C} 4-\mathrm{H} 4$ & 118.2 \\
\hline $\mathrm{O} 1-\mathrm{S} 1-\mathrm{N} 1$ & $104.16(7)$ & $\mathrm{C} 6-\mathrm{C} 5-\mathrm{C} 10$ & $119.86(14)$ \\
\hline $\mathrm{O} 2-\mathrm{S} 1-\mathrm{N} 1$ & $114.03(7)$ & $\mathrm{C} 6-\mathrm{C} 5-\mathrm{S} 1$ & $121.69(12)$ \\
\hline $\mathrm{O} 1-\mathrm{S} 1-\mathrm{C} 5$ & $107.67(7)$ & $\mathrm{C} 10-\mathrm{C} 5-\mathrm{S} 1$ & $118.44(12)$ \\
\hline $\mathrm{O} 2-\mathrm{S} 1-\mathrm{C} 5$ & $107.43(7)$ & $\mathrm{C} 7-\mathrm{C} 6-\mathrm{C} 5$ & $120.08(14)$ \\
\hline $\mathrm{N} 1-\mathrm{S} 1-\mathrm{C} 5$ & $108.97(7)$ & $\mathrm{C} 7-\mathrm{C} 6-\mathrm{H} 6$ & 120.0 \\
\hline $\mathrm{C} 11-\mathrm{O} 3-\mathrm{H} 1 \mathrm{H}$ & $108.9(16)$ & $\mathrm{C} 5-\mathrm{C} 6-\mathrm{H} 6$ & 120.0 \\
\hline $\mathrm{H} 1 \mathrm{~W}-\mathrm{O} 1 \mathrm{~W}-\mathrm{H} 2 \mathrm{~W}$ & $101(2)$ & $\mathrm{C} 6-\mathrm{C} 7-\mathrm{C} 8$ & $120.63(15)$ \\
\hline $\mathrm{C} 1-\mathrm{N} 1-\mathrm{S} 1$ & $121.16(11)$ & $\mathrm{C} 6-\mathrm{C} 7-\mathrm{H} 7$ & 119.7 \\
\hline $\mathrm{C} 2-\mathrm{N} 2-\mathrm{C} 1$ & $116.93(14)$ & $\mathrm{C} 8-\mathrm{C} 7-\mathrm{H} 7$ & 119.7 \\
\hline $\mathrm{C} 4-\mathrm{N} 3-\mathrm{C} 1$ & $116.59(13)$ & $\mathrm{N} 4-\mathrm{C} 8-\mathrm{C} 7$ & $120.77(15)$ \\
\hline $\mathrm{C} 8-\mathrm{N} 4-\mathrm{H} 1 \mathrm{~N}$ & $113.6(15)$ & $\mathrm{N} 4-\mathrm{C} 8-\mathrm{C} 9$ & $120.40(15)$ \\
\hline $\mathrm{C} 8-\mathrm{N} 4-\mathrm{H} 2 \mathrm{~N}$ & $114.6(15)$ & $\mathrm{C} 7-\mathrm{C} 8-\mathrm{C} 9$ & $118.78(14)$ \\
\hline $\mathrm{H} 1 \mathrm{~N}-\mathrm{N} 4-\mathrm{H} 2 \mathrm{~N}$ & $116(2)$ & $\mathrm{C} 10-\mathrm{C} 9-\mathrm{C} 8$ & $120.39(14)$ \\
\hline $\mathrm{C} 12-\mathrm{N} 5-\mathrm{H} 3 \mathrm{~N}$ & $111.5(13)$ & $\mathrm{C} 10-\mathrm{C} 9-\mathrm{H} 9$ & 119.8 \\
\hline $\mathrm{C} 12-\mathrm{N} 5-\mathrm{H} 4 \mathrm{~N}$ & $111.2(14)$ & $\mathrm{C} 8-\mathrm{C} 9-\mathrm{H} 9$ & 119.8 \\
\hline $\mathrm{H} 3 \mathrm{~N}-\mathrm{N} 5-\mathrm{H} 4 \mathrm{~N}$ & $104.9(19)$ & $\mathrm{C} 9-\mathrm{C} 10-\mathrm{C} 5$ & $120.26(14)$ \\
\hline $\mathrm{C} 12-\mathrm{N} 5-\mathrm{H} 5 \mathrm{~N}$ & $114.0(13)$ & $\mathrm{C} 9-\mathrm{C} 10-\mathrm{H} 10$ & 119.9 \\
\hline $\mathrm{H} 3 \mathrm{~N}-\mathrm{N} 5-\mathrm{H} 5 \mathrm{~N}$ & $107.1(18)$ & $\mathrm{C} 5-\mathrm{C} 10-\mathrm{H} 10$ & 119.9 \\
\hline $\mathrm{H} 4 \mathrm{~N}-\mathrm{N} 5-\mathrm{H} 5 \mathrm{~N}$ & $107.5(19)$ & $\mathrm{O} 3-\mathrm{C} 11-\mathrm{C} 12$ & $111.39(13)$ \\
\hline $\mathrm{N} 3-\mathrm{C} 1-\mathrm{N} 2$ & $124.11(14)$ & $\mathrm{O} 3-\mathrm{C} 11-\mathrm{H} 11 \mathrm{~A}$ & 109.4 \\
\hline $\mathrm{N} 3-\mathrm{C} 1-\mathrm{N} 1$ & $122.02(13)$ & $\mathrm{C} 12-\mathrm{C} 11-\mathrm{H} 11 \mathrm{~A}$ & 109.4 \\
\hline $\mathrm{N} 2-\mathrm{C} 1-\mathrm{N} 1$ & $113.87(14)$ & $\mathrm{O} 3-\mathrm{C} 11-\mathrm{H} 11 \mathrm{~B}$ & 109.4 \\
\hline $\mathrm{N} 2-\mathrm{C} 2-\mathrm{C} 3$ & $122.86(15)$ & $\mathrm{C} 12-\mathrm{C} 11-\mathrm{H} 11 \mathrm{~B}$ & 109.3 \\
\hline $\mathrm{N} 2-\mathrm{C} 2-\mathrm{H} 2$ & 118.6 & $\mathrm{H} 11 \mathrm{~A}-\mathrm{C} 11-\mathrm{H} 11 \mathrm{~B}$ & 108.0 \\
\hline
\end{tabular}


1443

1444

1445

1446

1447

1448

1449

1450

1451

1452

1453

1454

1455

1456

1457

1458

1459

1460

1461

1462

1463

1464

1465

1466

1467

1468

1469

1470

1471

1472

1473

1474

1475

1476

1477

$\begin{array}{ll}\mathrm{C} 3-\mathrm{C} 2-\mathrm{H} 2 & 118.6 \\ \mathrm{C} 4-\mathrm{C} 3-\mathrm{C} 2 & 115.90(15) \\ \mathrm{C} 4-\mathrm{C} 3-\mathrm{H} 3 & 122.1 \\ \mathrm{C} 2-\mathrm{C} 3-\mathrm{H} 3 & 122.1 \\ \mathrm{~N} 3-\mathrm{C} 4-\mathrm{C} 3 & 123.61(15) \\ \mathrm{N} 3-\mathrm{C} 4-\mathrm{H} 4 & 118.2 \\ & \\ \mathrm{O} 1-\mathrm{S} 1-\mathrm{N} 1-\mathrm{C} 1 & 178.29(12) \\ \mathrm{O} 2-\mathrm{S} 1-\mathrm{N} 1-\mathrm{C} 1 & 52.98(14) \\ \mathrm{C} 5-\mathrm{S} 1-\mathrm{N} 1-\mathrm{C} 1 & -67.02(14) \\ \mathrm{C} 4-\mathrm{N} 3-\mathrm{C} 1-\mathrm{N} 2 & 0.3(2) \\ \mathrm{C} 4-\mathrm{N} 3-\mathrm{C} 1-\mathrm{N} 1 & -179.55(14) \\ \mathrm{C} 2-\mathrm{N} 2-\mathrm{C} 1-\mathrm{N} 3 & -0.1(2) \\ \mathrm{C} 2-\mathrm{N} 2-\mathrm{C} 1-\mathrm{N} 1 & 179.74(13) \\ \mathrm{S} 1-\mathrm{N} 1-\mathrm{C} 1-\mathrm{N} 3 & -4.7(2) \\ \mathrm{S} 1-\mathrm{N} 1-\mathrm{C} 1-\mathrm{N} 2 & 175.48(10) \\ \mathrm{C} 1-\mathrm{N} 2-\mathrm{C} 2-\mathrm{C} 3 & -0.2(2) \\ \mathrm{N} 2-\mathrm{C} 2-\mathrm{C} 3-\mathrm{C} 4 & 0.3(2) \\ \mathrm{C} 1-\mathrm{N} 3-\mathrm{C} 4-\mathrm{C} 3 & -0.1(2) \\ \mathrm{C} 2-\mathrm{C} 3-\mathrm{C} 4-\mathrm{N} 3 & -0.1(2) \\ \mathrm{O} 1-\mathrm{S} 1-\mathrm{C} 5-\mathrm{C} 6 & -138.00(12) \\ \mathrm{O} 2-\mathrm{S} 1-\mathrm{C} 5-\mathrm{C} 6 & -14.40(14) \\ & \end{array}$

N5-C12-C11

N5-C12-H12A

$\mathrm{C} 11-\mathrm{C} 12-\mathrm{H} 12 \mathrm{~A}$

N5-C12-H12B

C11-C12-H12B

$\mathrm{H} 12 \mathrm{~A}-\mathrm{C} 12-\mathrm{H} 12 \mathrm{~B}$

$\mathrm{N} 1-\mathrm{S} 1-\mathrm{C} 5-\mathrm{C} 6$

$\mathrm{O} 1-\mathrm{S} 1-\mathrm{C} 5-\mathrm{C} 10$

$\mathrm{O} 2-\mathrm{S} 1-\mathrm{C} 5-\mathrm{C} 10$

$\mathrm{N} 1-\mathrm{S} 1-\mathrm{C} 5-\mathrm{C} 10$

$\mathrm{C} 10-\mathrm{C} 5-\mathrm{C} 6-\mathrm{C} 7$

$\mathrm{S} 1-\mathrm{C} 5-\mathrm{C} 6-\mathrm{C} 7$

$\mathrm{C} 5-\mathrm{C} 6-\mathrm{C} 7-\mathrm{C} 8$

$\mathrm{C} 6-\mathrm{C} 7-\mathrm{C} 8-\mathrm{N} 4$

C6- $7-\mathrm{C} 8-\mathrm{C} 9$

N4-C8-C9-C10

$\mathrm{C} 7-\mathrm{C} 8-\mathrm{C} 9-\mathrm{C} 10$

$\mathrm{C} 8-\mathrm{C} 9-\mathrm{C} 10-\mathrm{C} 5$

C6-C5-C10-C9

$\mathrm{S} 1-\mathrm{C} 5-\mathrm{C} 10-\mathrm{C} 9$

$\mathrm{O} 3-\mathrm{C} 11-\mathrm{C} 12-\mathrm{N} 5$
109.89 (13)

109.7

109.7

109.7

109.7

108.2

109.60 (13)

43.52 (13)

167.11 (11)

-68.88 (13)

-0.3 (2)

-178.76 (12)

1.0 (2)

176.66 (15)

$-0.8(2)$

$-177.60(14)$

$-0.1(2)$

0.8 (2)

$-0.6(2)$

177.88 (11)

56.48 (18)

Hydrogen-bond geometry $\left(\AA,{ }^{\circ}\right)$

\begin{tabular}{lllll}
\hline$D-\mathrm{H} \cdots A$ & $D-\mathrm{H}$ & $\mathrm{H} \cdots A$ & $D \cdots A$ & $D-\mathrm{H} \cdots A$ \\
\hline $\mathrm{N} 4-\mathrm{H} 1 N \cdots \mathrm{O} 1 W^{\mathrm{i}}$ & $0.89(2)$ & $2.34(2)$ & $3.173(2)$ & $156(2)$ \\
$\mathrm{N} 4-\mathrm{H} 2 N \cdots \mathrm{O} 1 W^{\mathrm{ii}}$ & $0.83(2)$ & $2.58(2)$ & $3.293(2)$ & $144.1(19)$ \\
$\mathrm{N} 5-\mathrm{H} 3 N \cdots \mathrm{O} 3^{\text {iii }}$ & $0.86(2)$ & $2.01(2)$ & $2.7762(18)$ & $147.2(18)$ \\
$\mathrm{N} 5-\mathrm{H} 4 N \cdots \mathrm{O} 2^{\text {ii }}$ & $0.90(2)$ & $1.99(2)$ & $2.8488(18)$ & $159(2)$ \\
$\mathrm{N} 5-\mathrm{H} 4 N \cdots \mathrm{N} 3^{\text {ii }}$ & $0.90(2)$ & $2.51(2)$ & $3.038(2)$ & $118.1(17)$ \\
$\mathrm{N} 5-\mathrm{H} 5 N \cdots \mathrm{O} 1^{\text {iv }}$ & $0.93(2)$ & $2.44(2)$ & $3.1006(18)$ & $128.4(17)$ \\
$\mathrm{N} 5-\mathrm{H} 5 N \cdots \mathrm{N} 1^{\text {iv }}$ & $0.93(2)$ & $2.02(2)$ & $2.933(2)$ & $167.3(19)$ \\
$\mathrm{O} 3-\mathrm{H} 1 H \cdots \mathrm{N} 2^{\mathrm{v}}$ & $0.84(3)$ & $1.90(3)$ & $2.7399(19)$ & $177(2)$ \\
$\mathrm{O} 1 W-\mathrm{H} 1 W \cdots \mathrm{O} 2$ & $0.97(3)$ & $1.94(3)$ & $2.9039(17)$ & $174(3)$ \\
$\mathrm{O} 1 W-\mathrm{H} 2 W \cdots \mathrm{O} 1^{\text {vi }}$ & $0.86(3)$ & $2.07(3)$ & $2.8997(18)$ & $163(2)$
\end{tabular}

Symmetry codes: (i) $-x,-y,-z+1$; (ii) $x,-y-1 / 2, z-1 / 2$; (iii) $-x+1,-y-1,-z+1$; (iv) $x, y-1, z$; (v) $-x+1,-y,-z+1$; (vi) $x,-y+1 / 2, z+1 / 2$. 


\section{${ }^{1478}$ other supporting information}

1479

1480

1481

1482

1483

1484

1485

Crystallographic Information File. uk3153.cif

Structure factors. uk3153Isup2.hk1

Structure factors. uk3153IIsup3.hk1

Structure factors. uk3153IIIsup4.hk1

Structure factors. uk3153IVsup5.hkl

Structure factors. uk3153Vsup6.hkl

Structure factors. uk3153VIsup7.hkl 